NASA CONTRACTOR REPOR T

$\stackrel{0}{2}$
$\vdots$
$\infty$
$\vdots$
$\vdots$
$\Sigma$

ROAN COPY: RETURN TO ABWL (WLit-2)

RIRTLAND AFB, N MEX

\title{
EFFECTS AND IMPORTANCE OF PENETRATION AND GROWTH OF LIFT ON SPACE VEHICLE RESPONSE
}

by Robert R. Blackburn and A. D. St. John

Prepared under Contract No. NAS 8-11012 by

MIDWEST RESEARCH INSTITUTE

Kansas City, Mo.

for

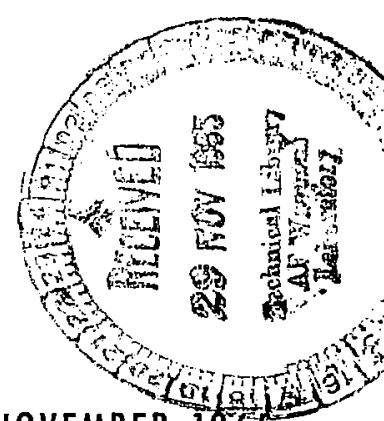

NATIONAL AERONAUTICS AND SPACE ADMINISTRATION - WASHINGTON, D. C. - NOVEMBER 1965 
NASA CR-326

Distribution of this report is provided in the interest of information exchange. Responsibility for the contents resides in the author or organization that prepared it.

Prepared under Contract No. NAS 8-11012 by

MIDWEST RESEARCH INSTITUTE

Kansas City, Mo.

for

\section{NATIONAL AERONAUTICS AND SPACE ADMINISTRATION}

For sale by the Clearinghouse for Federal Scientific and Technical Information Springfield, Virginia 22151 - Price $\$ 5.00$ 


\section{PREFACE}

This report was prepared by Midwest Research Institute under Contract No. NAS8-11012. The work was performed under the technical supervision of Mr. M. H. Rheinfurth, staff member of the Dynamics and Flight Mechanics Division, Marshall Space Flight Center.

This report covers work on the contract during the contract period 21 June 1963 to 20 August 1964 .

Mr. Robert R. Blackburn was the principal investigator for the analysis in this volume. Mr. A. D. St. John was project leader. Several members of the staff contributed in the preparation of numerical results: Messrs. Donald R. Kobett, John E. Scheu, Duncan Sommerville, and Dr. William D. Glauz. 

Notation . . . . . . . . . . . . . . . . . . . . . . . Ix

Abstract . . . . . . . . . . . . . . . . . . . . . $x v$

Summary . . . . . . . . . . . . . . . . . . . . . . . 1

I. Introduction .......................... 2

II. Baslc Goal of the Investigation . . . . . . . . . . . . . 2

III. Discussion of the Investigation . . . . . . . . . . . . . 3

A. Description of Saturn C-5 Model Used . . . . . . . . 3

B. Description of Indicial and Impulsive Aerodynamic
Forces Used . . . . . . . . . . . . . . 3

C. Method of Solution for Indicial and Impulsive

Responses of Saturn C-5 . . . . . . . . . . . . 10

D. Wind-Induced Responses . . . . . . . . . . . . 32

IV. Method Used in Analysis of Effects and Importance of Penetration and Growth of Lift on Missile Response . . . . . . . . . . 35

V. Results and Discussion . . . . . . . . . . . . . . 49

VI. Conclusions and Recommendations . . . . . . . . . . . . . 54

Bibliography . . . . . . . . . . . . . . . . . . . . . 59

Appendix I - Equations of Motion of Saturn C-5 . . . . . . . . 60

Appendix II - Development of Transient, Quasi-Steady and Steady Generalized Aerodynamic Force Expressions Resulting From a Unit Step and Unit Impulse Wind Profile . . . 70

Appendix III - Calculation of Initial and Steady-State Conditions Resulting From a Unit Impulse and Unit Step Wind Profile . . . . . . . . . . . . . . . . .

Appendix IV - Presentation of Extreme Excursion and Average Response

Plots................... 108 
TABLE OF CONIENTS (Continued)

\section{Iist of Tables}

Table No.

Title

Page No.

I

II

III

IV

VI

VII

VIII

IX

$\mathrm{X}$

XI

XII

Tabulation of Increment Sizes Used in Indicial and Impulsive Response Calculations . . . . . . . . . 12 Altitude Bands Used in Calculating Wind-Induced Responses . . . . . . . . . . . . . . 34

Ratios of Average Responses, Observed Maximum Responses and Expected Maximum Responses for Profile No. 1................. 50

Ratios of Average Responses, Observed Maximum Responses and Expected Maximum Responses for Profile No. 2. . . . . . . . . . . 51

Definition of Saturn C-5 Body Geometry . . . . . . . 77

Definition of Coefficients in (II-18), (II-25), and

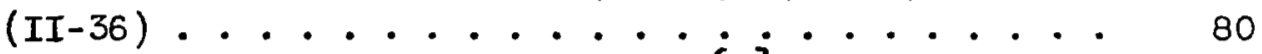

Elements of Forcing Function Matrix $\{\mathrm{C}\}$....... 100 Elernents of the Transformed Forcing Function

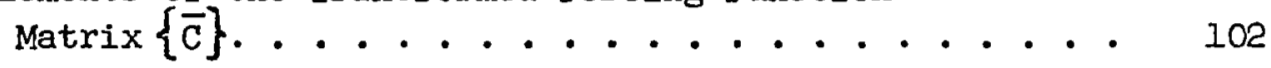

Initial Conditions Imposed by a Unit Impulse Vind

For the Cases of Penetration with Lift Growth and Pure Penetration ............ 103

Initial Conditions Iruposed by a Unit Step Wind for the Cases of Penetration with Lift Growth, Pure Penetration and Instantaneous Immersion . . . . . 105 Steady-State Values for Impulsive Responses . . . . . 107 Steady-State Values for Indicial Responses . . . . . 107 
TABLE - OF CONTENTS (Concluded)

\section{Iist of Figures}

Figure No.

Title

Page No.

1

2

3

4

5

6

$7-24$

25-33

34

35

36

37

38

39

40

41

42-89

90-99

Indicial Normal Force Growth Functions vs. Time . . . .

Indicial lst Bending Moment Growth Functions vs. Time . . Indicial and Bending Moment Growth Functions vs. Time . Impulsive Normal Force Growth Functions vs. Time . . . . 8 Impulsive lst Bending Moment Growth Functions vs. Time 9

Impulsive and Bending Moment Growth Functions vs. Time . 9

9 Impulsive and indicial responses of several generalized coordinates considering three aerodynamic representations - $70 \mathrm{sec}$. F.T. . . . . . . . . . . . .

Wind-induced responses of several generalized coordinates considering three aerodynamic representations - $70 \mathrm{sec}$. F.T. band . . . . . . . . . . .

Extreme Excursion of and Bending vs. Cumulative Probability - $60 \mathrm{sec}$. F.T. . . . . . . . . . . . 48

Extreme Excursion of and Bending vs. Reduced Cumulative Probability - $60 \mathrm{sec}$. F.T. . . . . . . . . . . . Approximate 4th Bending Impulsive Response vs. Response Time - $60 \mathrm{sec}$. F.T.

Approximate 2nd Bending Impulsive Response vs. Response Time - $60 \mathrm{sec}$. F.T.

Actual 2nd Bending Impulsive Response vs. Response Time - $60 \mathrm{sec}$. F.T. . . . . . . . . . . . . 57 Saturn C-5 Coordinate System . . . . . . . . . . 61 Mechanical Analogy or Sloshing Fluids . . . . . . . . 62 Saturn C-5 Body Geometry . . . . . . . . . . . . 72 Extreme excursion of various generalized coordinates vs. reduced cumulative probability for five flight time bands . . . . . . . . . . . . . 109-135 Average response of various generalized coordinates vs. flight time................. 


\section{NOTATION}

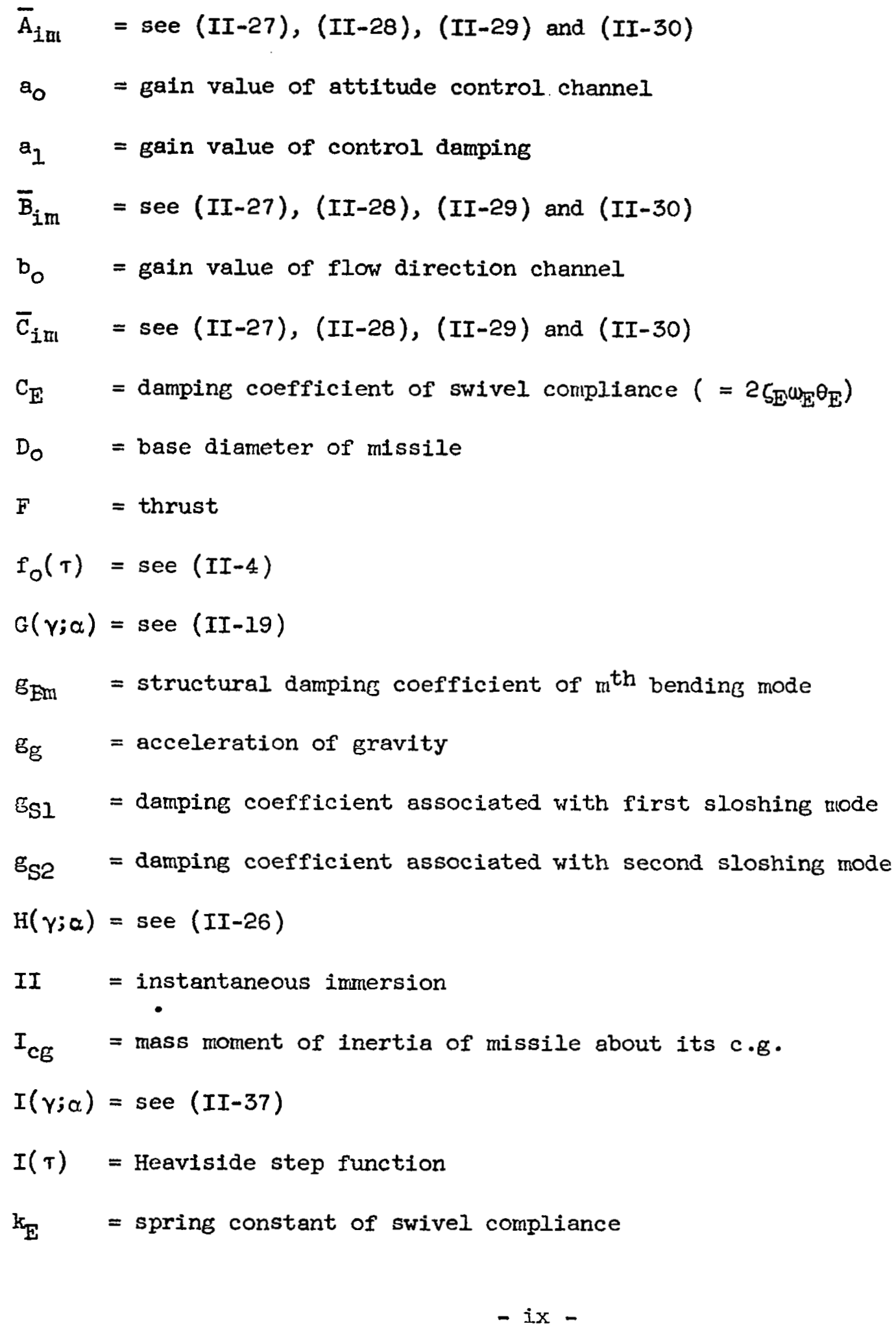




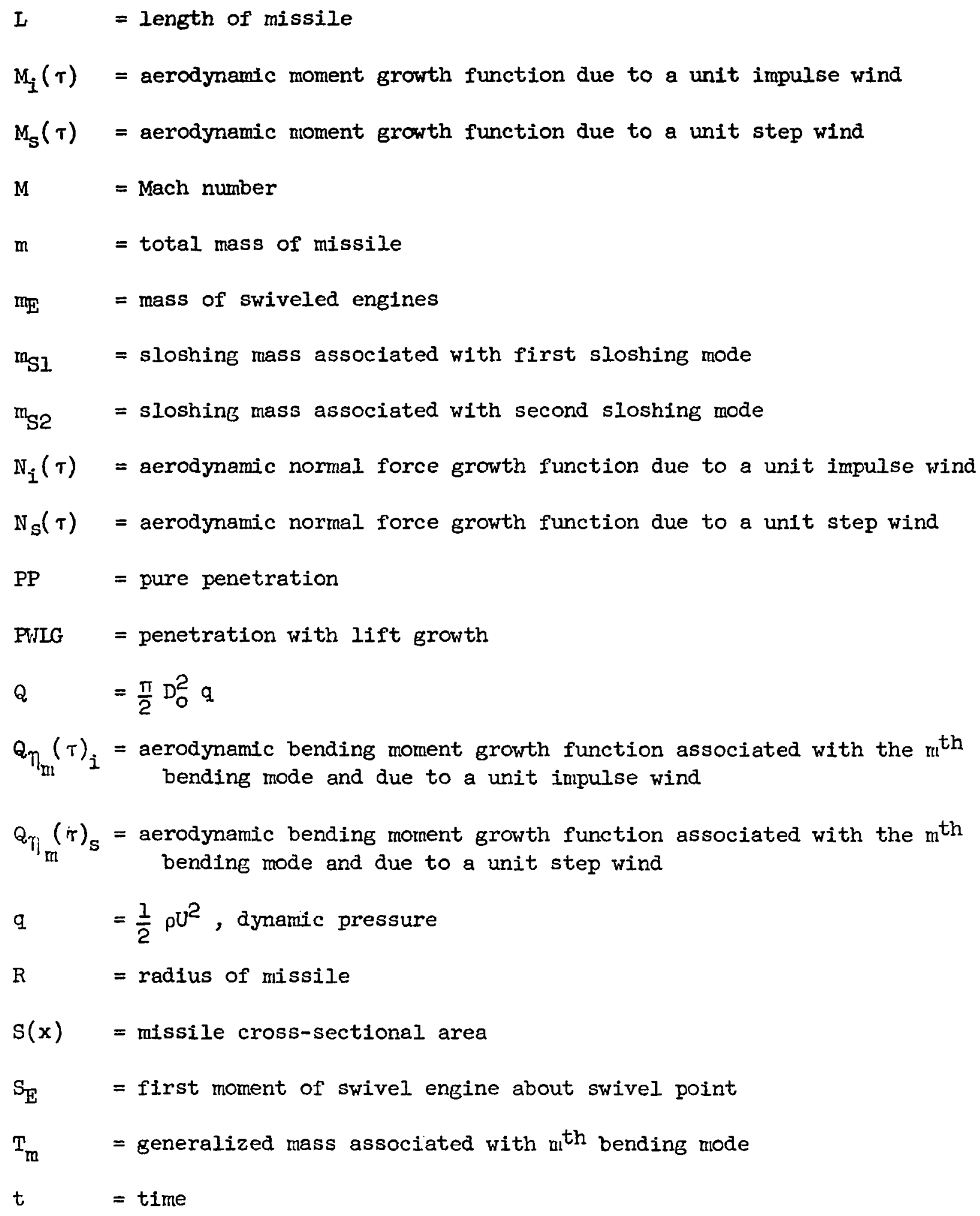


= missile free stream velocity

$v_{y}, v(x, t)=$ horizontal wind velocity

$\mathrm{x}_{\mathrm{g}} \quad=$ coordinate of missile $c \cdot g$.

$x_{E} \quad=$ coordinate of gimbal point

$\mathrm{x}_{\mathrm{S} I} \quad=$ coordinate of sloshing mass associated with first sloshing mode

$\mathrm{x}_{\mathrm{S} 2} \quad=$ coordinate of sloshing mass associated with second sloshing mode

$x_{T} \quad=$ coordinate of top of missile

$Y_{\mathrm{m}} \quad=\mathrm{m}^{\text {th }}$ bending mode deflection curve (normalized to one at the gimbal point)

$\mathrm{y}_{0} \quad=$ lateral translation of rigid mode

Aerodymamic Integrals

$A_{\mathrm{mi}}=-\int_{-I / 2}^{L / 2} Y_{m}(x)\left[2 \lambda(x) \lambda^{\prime}(x) Y_{i}^{\prime}(x)+\lambda^{2}(x) Y_{i}^{\prime \prime}(x)\right] d x \quad(m, i=1,2,3,4)$

$B_{\mathrm{n} i \mathrm{i}}=-2 \int_{-L / 2}^{\mathrm{I} / 2} \mathrm{Y}_{\mathrm{m}}(\mathrm{x})\left[\lambda(\mathrm{x}) \lambda^{\prime}(\mathrm{x}) \mathrm{Y}_{i}(\mathrm{x})+\lambda^{2}(\mathrm{x}) \mathrm{Y}_{i}^{\prime}(\mathrm{x})\right] \mathrm{dx} \quad(\mathrm{m}, \dot{i}=1,2,3,4)$

$C_{m i}=\int_{-I / 2}^{L / 2} \lambda^{2}(x) Y_{m}(x) Y_{i}(x) d x$

$(\mathrm{m}, i=1,2,3,4)$

$D_{I u}=\int_{-L / 2}^{L / 2} \lambda(x) \lambda^{\prime}(x) Y_{m}(x) d x$

$(\pi=1,2,3,4)$

$\bar{D}_{m}=\int_{-L / 2}^{L / 2} \lambda(x) \lambda^{\prime}(x)\left(x-x_{G}\right) Y_{m}(x) d x$

$(m=1,2,3,4)$

$E_{m}=\int_{-L / 2}^{L / 2}\left[2 \lambda(x) \lambda^{\prime}(x) Y_{m}^{\prime}+\lambda^{2}(x) Y_{m}^{\prime \prime}(x)\right] d x \quad(m=1,2,3,4)$ 


$$
\begin{aligned}
& \bar{E}_{m}=-\int_{-L / 2}^{I / 2}\left[2 \lambda(x) \lambda^{\prime}(x) Y_{m}^{\prime}(x)+\lambda^{2}(x) Y_{m l}^{\prime \prime}(x)\right]\left(x-x_{g}\right) d x \quad(m=1,2,3,4) \\
& F_{k}=\int_{-L / 2}^{L / 2} \lambda(x) \lambda^{\prime}(x)\left(x-x_{g}\right)^{k} d x \quad(k=0,1,2) \\
& G_{m}=\int_{-L / 2}^{L / 2} \lambda^{2}(x) Y_{m}(x) d x \quad(m=1,2,3,4) \\
& \bar{G}_{m}=\int_{-L / 2}^{L / 2} \lambda^{2}(x)\left(x-x_{g}\right) Y_{m}(x) d x \quad(m=1,2,3,4) \\
& \mathrm{H}_{\mathrm{m}}=\int_{-\mathrm{L} / 2}^{\mathrm{L} / 2} \lambda^{2}(\mathrm{x}) \mathrm{Y}_{\mathrm{m}}^{\prime}(\mathrm{x}) \mathrm{d} \mathrm{x} \quad(\mathrm{m}=1,2,3,4) \\
& \overline{\mathrm{H}}_{\mathrm{m}}=\int_{-\mathrm{L} / 2}^{\mathrm{I} / 2} \lambda^{2}(\mathrm{x})\left(\mathrm{x}-\mathrm{x}_{\mathrm{g}}\right) \mathrm{Y}_{\mathrm{m}}^{\prime}(\mathrm{x}) \mathrm{dx} \quad \quad(\mathrm{In}=1,2,3,4) \\
& J_{k}=\int_{-L / 2}^{L / 2} \lambda^{2}(x)\left(x-x_{g}\right)^{3 k} d x \quad(k=0,1,2)
\end{aligned}
$$

\section{Greek Symbols}

$$
\begin{aligned}
& \alpha_{i}=\text { indicated angle of attack } \\
& \beta_{C}=\text { control deflection (sometimes referred to as } i \text {, first control) } \\
& \beta_{E}=\text { actual swivel engine deflection } \\
& \zeta_{E}=\text { damping ratio of swivel engine } \\
& \eta_{m}=\text { amplitude of the } m^{\text {th }} \text { bending mode at gimbal point } \\
& \theta_{E}=\text { mass moment of inertia of swivel engine about swivel point } \\
& \lambda(x)=\frac{2 R(x)}{D_{0}}
\end{aligned}
$$




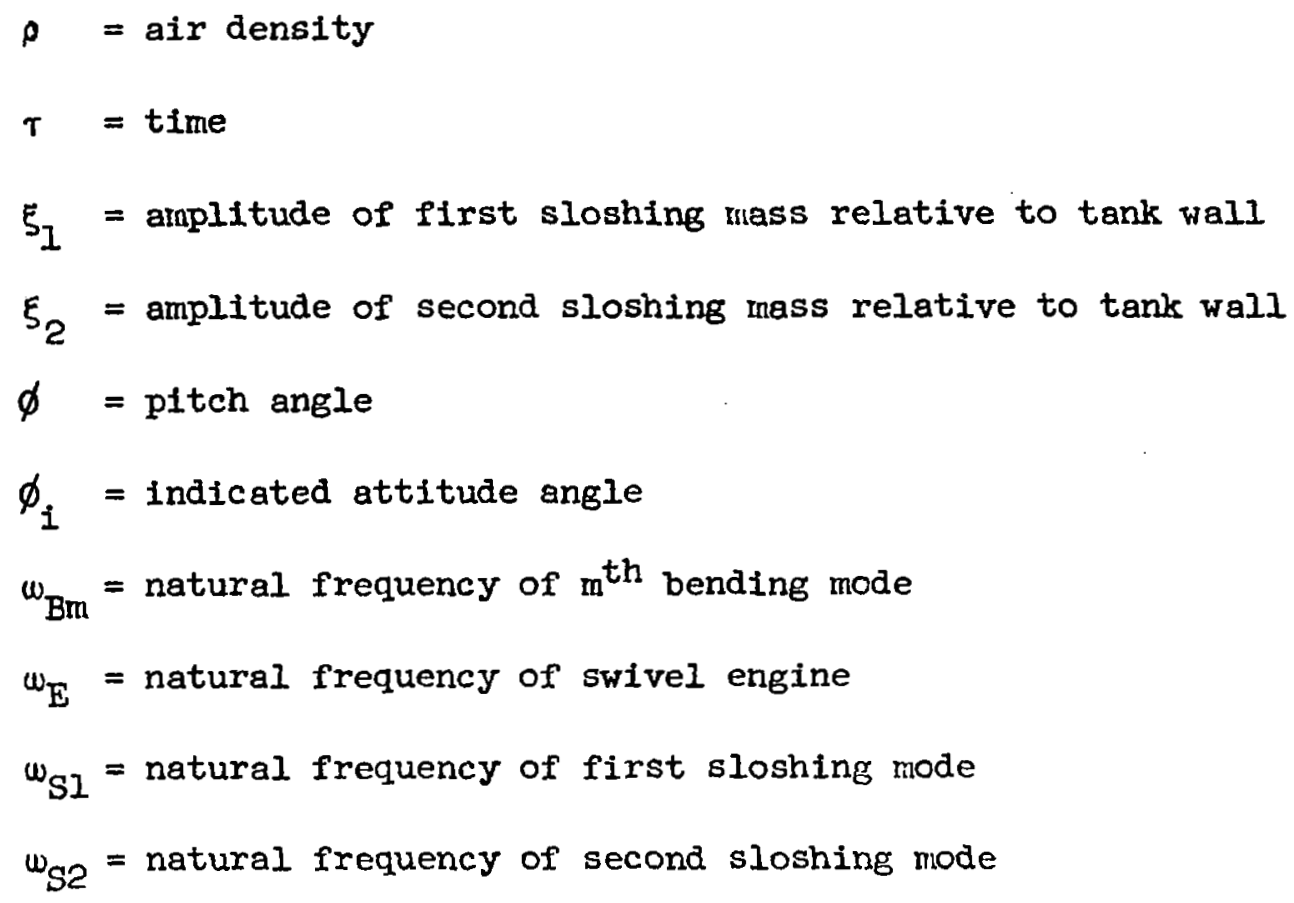




\section{ABSTRACT}

The wind induced responses of the Saturn C-5 without fins are calculated with three aerodynamic representations. The most accurate representation uses unsteady aerodynamics and accounts for penetration into the gusts. The second uses pseudo steady aerodynamics and accounts for penetration. The third uses pseudo steady aerodynamics and assumes equal wind cross flows over the missile length.

The responses, which include two sloshing and two bending modes, are affected by penetration to a detectable but insignificant degree. The use of unsteady aerodynamics causes very little change. The conventional, third aerodynamic representation provided in these cases slightly conservative (large) responses. 


\section{SUMMARY}

Missile responses to winds are usually calculated using pseudo steady, slender body aerodymamics and the assumption that wind induced crossflows are equal at all stations along the missile. However, it has been recognized that the time delays associated with missile penetration into a gust could significantly affect the actual forcing functions, especially those for the bending modes.

The wind induced responses of the Saturn C-5 (without fins) are calculated and compared for three aerodynamic representations. The most accurate representation accounts for the time delays of penetration and uses unsteady aerodynamics so that growth of lift smoothing and delays are included. The second representation, called pure penetration, uses pseudo steady aerodynamics but includes penetration delays. The third representation is the conventional one, defined here as instantaneous immersion.

The analytical model includes the following degrees of freedom: translation, rotation, first and second bending, two sloshing modes, and control. Frozen coefficients are used with each set applicable to a 10-sec. flight time.

Responses are calculated for the unit step and unit impulse gusts. These responses are used in a Duhamel integration to obtain the responses to two wind profiles defined by values at 25 -meter altitude increments.

The incorporation of penetration effects causes a detectable but insignificant change in the responses calculated for the Saturn C-5. The addition of growth of lift causes a very small change. The conventional, instantaneous immersion, responses are conservative (large) for the cases computed.

The present calculations do not provide an example of significant response changes due to penetration and lift growth. However, it appears that the possibility and nature of significant effects could be detected in a comparison of the unit step and impulse responses based on instantaneous immersion and pure penetration.

It is recommended that the response comparisons be extended to the third and fourth bending modes and to a Saturn C-5 with (simulated) fins. 


\section{INTRODUCTION}

The calculation of missile responses due to winds requires the incorporation of a satisfactory description of the aerodymamic forces into the response calculations. In a previous program [I], two aerodynamic effects were pointed out which have been neglected in previous investigations of missile response to horizontal winds or gusts. The first is the gust penetration effect and the second is the lift growth lag due to aerodynamic inertia. In the same reference these effects were incorporated in rigid body indicial and impulsive aerodynamic loads based on slender body theory. Some numerical results were presented for a multistage missile entering a unit step and impulse gust. The results, however, were not utilized in response calculations.

Some effects of penetration and growth of lift were postulated in [I]. For rigid body inotions, the effect will be to filter out the high wind frequencies. On the other hand, when missile bending modes are included, there is the possibility of augmenting some wind frequency responses.

The project reported here is an investigation of the effects and importance of penetration and growth of lift on missile responses when both rigid body and bending modes are considered. The Saturn C-5 configuration, excluding fins, is used in the study.

\section{BASIC GOAL OF THE IIVESTIGATION}

The basic goal of the work presented in this report was to determine the effects and importance of penetration and growth of lift on missile reponses. To accomplish this goal, the investigation was divided into two phases:

1. The indicial* and impulsive** responses of the vehicle were computed using three aerodynamic considerations: (1) penetration with lift growth effects, (2) pure penetration, and (3) instantaneous immersion. Slender body theory was used throughout the investigation.

2. The wind-induced responses of the vehicle for the three aerodynamic environments were computed from the impulsive responses using the Duhamel superposition integral.

* Response to a unit step.

* Response to a unit impulse. 
In both phases, numerical comparisons were made of responses calculated both with and without the effect of penetration and lift growth. A mathematical model of the Saturn C-5 configuration, excluding fins, was used in the analysis.

\section{DISCUSSION OF THE INVESTIGATION}

\section{A. Description of Saturn C-5 Model Used}

The Saturn C-5 configuration was used in the investigation of the effects and importance of penetration and growth of lift on missile response. The equations of motion of the missile system are presented in Appendix $I$. Seven generalized coordinates were considered in the response calculations: lateral translation, $\mathrm{y}_{0}$; rotation, $\phi$; the first two bending coordinates, $\eta_{1}$ and $\eta_{2}$; two sloshing coordinates, $\xi_{1}$ and $\xi_{2}^{*}$; and control deflection, $\beta_{C}^{* *}$. The control system considered in this report utilizes both an attitude reference control and a flow direction indicator.

The equations are valid for a swivel engine controlled vehicle where the swiveled engines account for four-fifths of the total thrust force. The missile and atmospheric parameters appearing in the equations are considered constant in predetermined time or altitude intervals.

The actual Saturn C-5 configuration contains englne shrouds and fins located on the aft section of the vehicle. The effects of these empennages were neglected in the analysis.

B. Description of Indicial and Impulsive Aerodynamic Forces Used

The development of the transient and quasi-steady, generalized aerodynamic forces resulting from a unit step and impulsive wind profile is presented in Appendix II of this report. The development is based on slender body theory. The forces corresponding to rigid body and bending coordinates are presented first for a general wind profile. The indicial and impulsive forces are then derived.

* The two sloshing coordinates considered are associated with the fundamental fluid motion in the lox and fuel tanks located in the booster stage of the $\mathrm{C}-5$.

** The actual engine deflection, $\beta_{\mathrm{E}}$, and the control deflection, $\beta_{\mathrm{C}}$, are considered to be equal. 
The wind-induced forcing functions which are compared in this reprt have both a geometric and an aerodynamic aspect.

In the geometric consideration two cases are used. In the simplest case, instantaneous immersion, all stations along the missile are assumed to be immersed in the same wind-induced crossflow, namely the wind crossflow occurring at the nose. The more accurate geometric representation, called penetration, assigns to each station along the missile the wind crossflow which exists at the altitude occupied by the station. With penetration, the missile nose enters a side gust first and in subsequent time successive stations along the missile length move into the crossflow.

Two representations of the aerodynamics are used; they are quasisteady and transient. In the quasi-steady representation the airforces at a missile station are those which would exist if the local crossflow persisted unchanged for an extended time. The transient representation is based on the theory of unsteady motion of slender bodies and includes the growth of lift with time.

Three types of wind-induced forcing functions are assembled using combinations of the geometric and aerodynamic representations. The simplest type is called instantaneous immersion and uses the instantaneous inmersion geometric representation with quasi-steady aerodynamics. A more accurate type, called pure penetration, uses penetration geometrics and quasi-steady aerodynamics. The most accurate aerodynamic forcing functions are called penetration with lift growth. These latter functions use the penetration geonetrics with transient aerodynamics.*

The simpler function types, instantaneous immersion and pure penetration, can be obtained from penetration with lift growth \{see Appendix II .

The development of the indicial and impulsive transient and quasisteady generalized aerodynamic forces for rigld body motion follows the work presented in [1] and [2].

The derivation of the indicial and impulsive transient and quasisteady aerodymamic forces, $Q_{\eta}$, corresponding to the bending coordinates,

* The crossflows induced by missile motions are in all cases treated with quasi-steady aerodynamics. These crossflows are small in comparison to the wind-induced crossflows and appear in the left hand side of the equations of motion $\{$ see Appendix I \} . 
$\eta_{\mathrm{m}}^{*}$, requires a description of the mode shapes of the missile. A considerable savings in computation can be obtained if the mode shapes, $Y_{m}(x)$, are approximated by polynomials. The components of $Q_{\eta_{\text {in }}}$ corresponding to the constant and linear terms of the polynomials can then be rewritten in terms of the windinduced aerodynanic force expressions corresponding to the rigid body coordinates. In addition, the polynomial representations for $Y_{m}(x)$ need only apply to specific regions of the missile length, since the kernel of the integrals describing $Q_{\eta_{m}}$ take on values only over the conic sections of the missile. The use of polynomial approximations for $Y_{m}(x)$ does not detract from a general approach to numerical solution, since the important mode shapes of the Saturn C-5 configuration were efficiently described by low order polymomials in the regions of interest.

Mode shape segments, corresponding to the conical regions of the C-5, were fitted with quadratic polynomials of the form

$$
Y_{m}(x)=\bar{A}_{i m}+\bar{B}_{i m} x+\bar{C}_{i m} x^{2}
$$

where the coefficients $\bar{A}, \bar{B}$ and $\bar{C}^{* *}$ are considered constant for a discrete flight time or altitude.

The subscripts $m$ and $i$ designate a specific mode and conical region, respectively. Four conical regions ( $i=1,2,3,4$ ) were considered in the analysis \{see Appendix II\}. For the C-5 configuration, the quadratic polynomial mode shapes yielded values which are within 1 per cent of the actual mode deflections.

* Penetration and lift growth lag effects are potentially important when missile bending modes are considered in an analysis of missile response. Since the wind-induced aerodynamic forces corresponding to the bending coordinates were not developed in [1], it was necessary to formulate these forces in this report. These forces were derived using slender body theory and include the geonetric and aerodynamic aspects discussed above.

* Numerical values of these coefficients were computed for the first four bending modes $(m=1,2,3,4)$ between flight times of 10 and $140 \mathrm{sec}$. at 10-sec. Intervals. However, values for only the first two bending modes between 30 and $100 \mathrm{sec}$, inclusive, were used in the numerical computation of the indicial and impulsive responses. 
The fundamental expressions required to compute the wind-induced forcing functions for the Saturn C-5 are given in Appendix II. The equation numbers of the indicial and impulsive forcing functions are given below for each of the three aerodynamic considerations.

\section{Aerodynamic Consideration}

1. Penetration with lift growth
Equation Numbers
a. Indicial
$(I I-13),(I I-20),(I I-31)$
b. Impulsive
$(I I-38),(I I-39),(I I-40)$

2. Fure penetration
a. Indicial
(II-5I), (II-53), (II-55)
b. Impulsive
(II-57), (II-59), (II-6I)

3. Instantaneous immersion
a. Indicial
$(I I-66),(I I-67),($ II-68)
b. Impulsive
$(I I-69),(I I-70),(I I-71)$

For purposes of general interest, plots of the C-5 normal force, first bending moment and second bending moment for a unit step and impulse side wind are given in this section. The curves are presented for a Mach number of 1.345 (70-sec. flight time). The consideration of penetration effects causes the generalized forces to be distributed over time. These timedependent forcing functions are called growth functions. The indicial normal force, first bending moment and second bending moment growth functions are given in Figs. 1, 2 and 3, respectively. The impulsive normal force, first bending moment and second bending moment growth functions are given in Figs. 4,5 and 6 , respectively. The effects of penetration with lift growth, pure penetration and instantaneous immersion on the generalized forces can be easily seen from the figures. The impulsive forces resulting from the effects of instantaneous immersion are not presented since they can only be described from a limit consideration. 


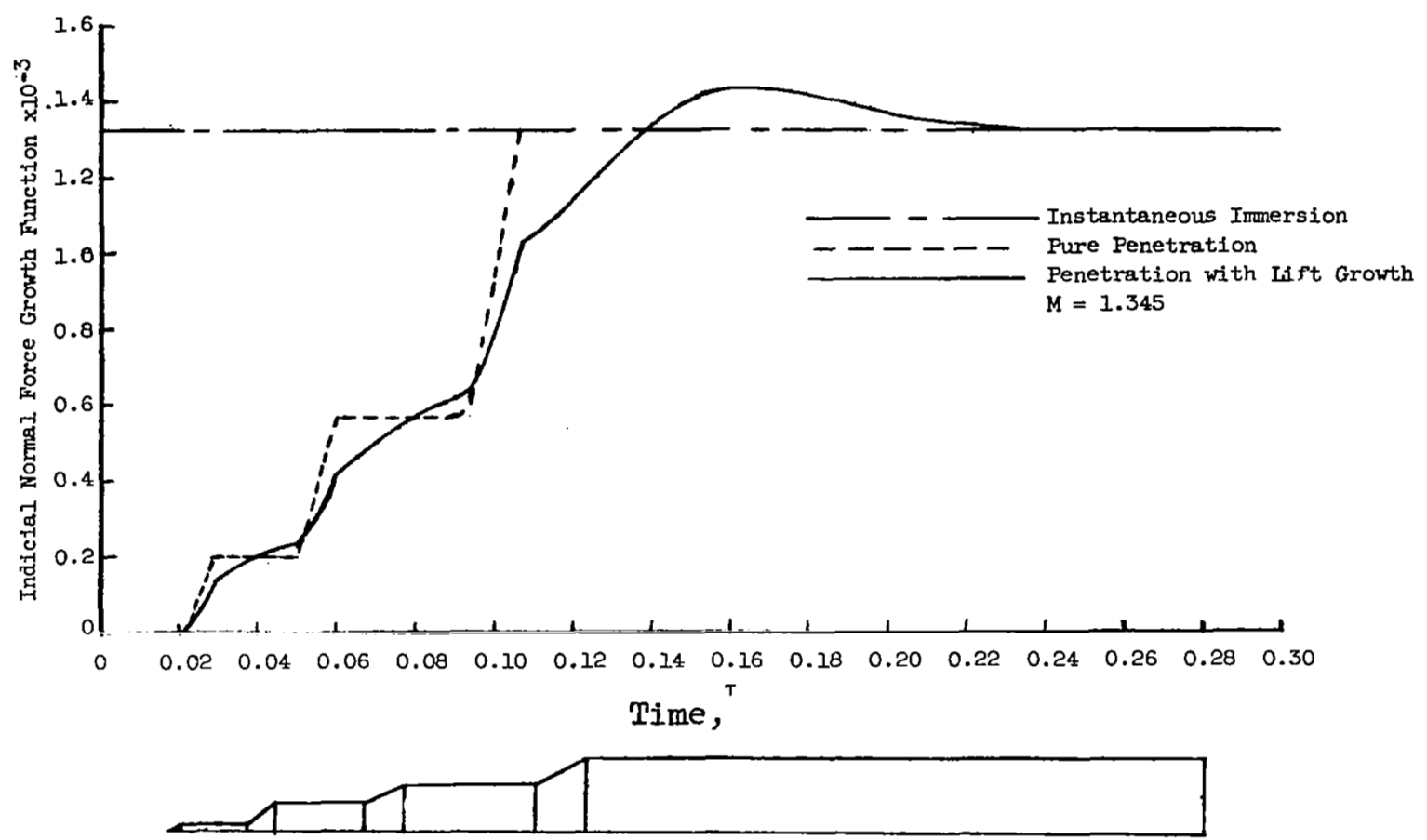

Fig. 1 - Indicial Normal Force Growth Functions vs. Time in Seconds

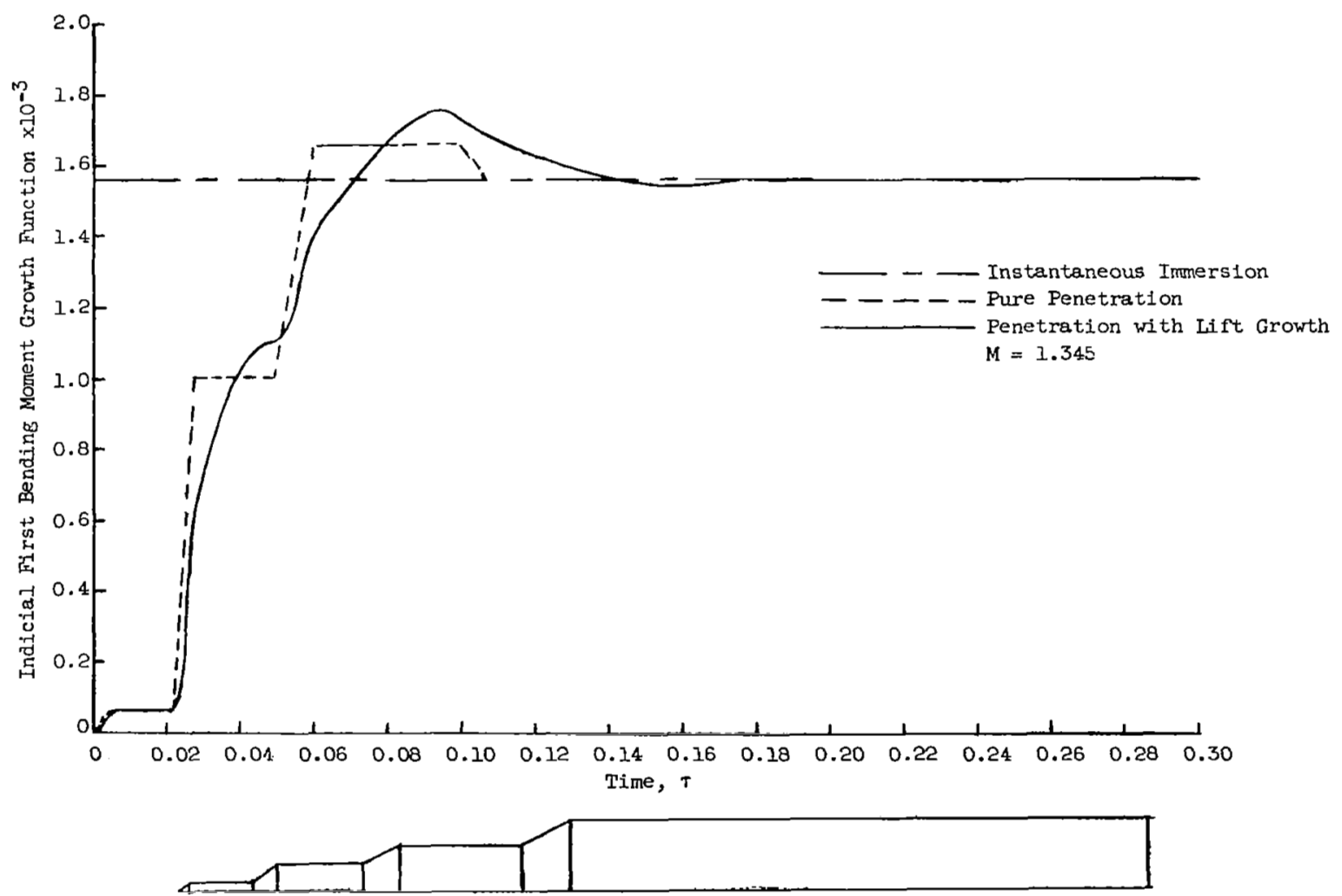

Fig. 2 - Indicial lst Bending Moment Growth Functions vs. Time in Seconds 

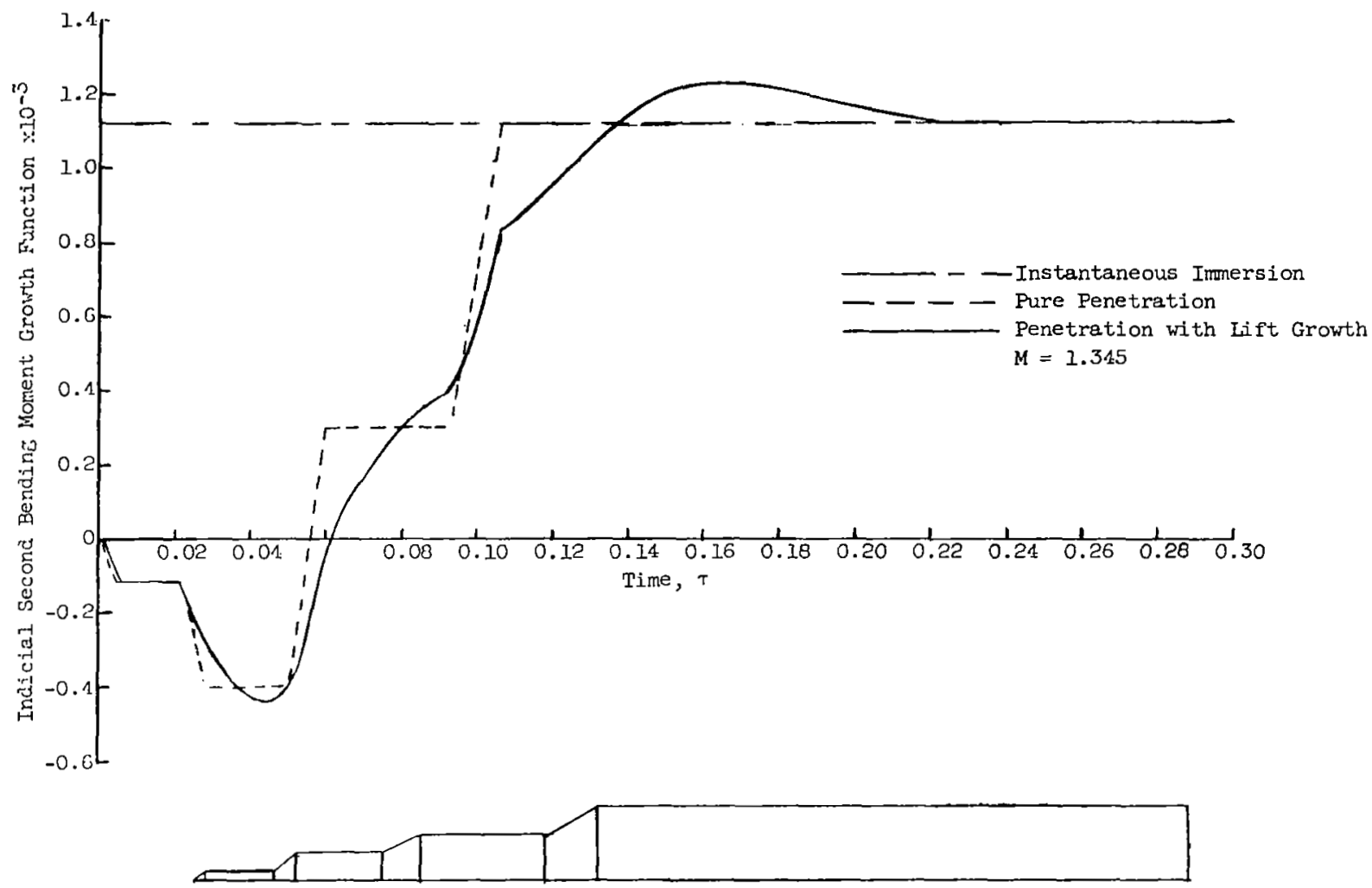

Fig. 3 - Indicial and Bending Moment Growth Functions vs. Time in Seconds
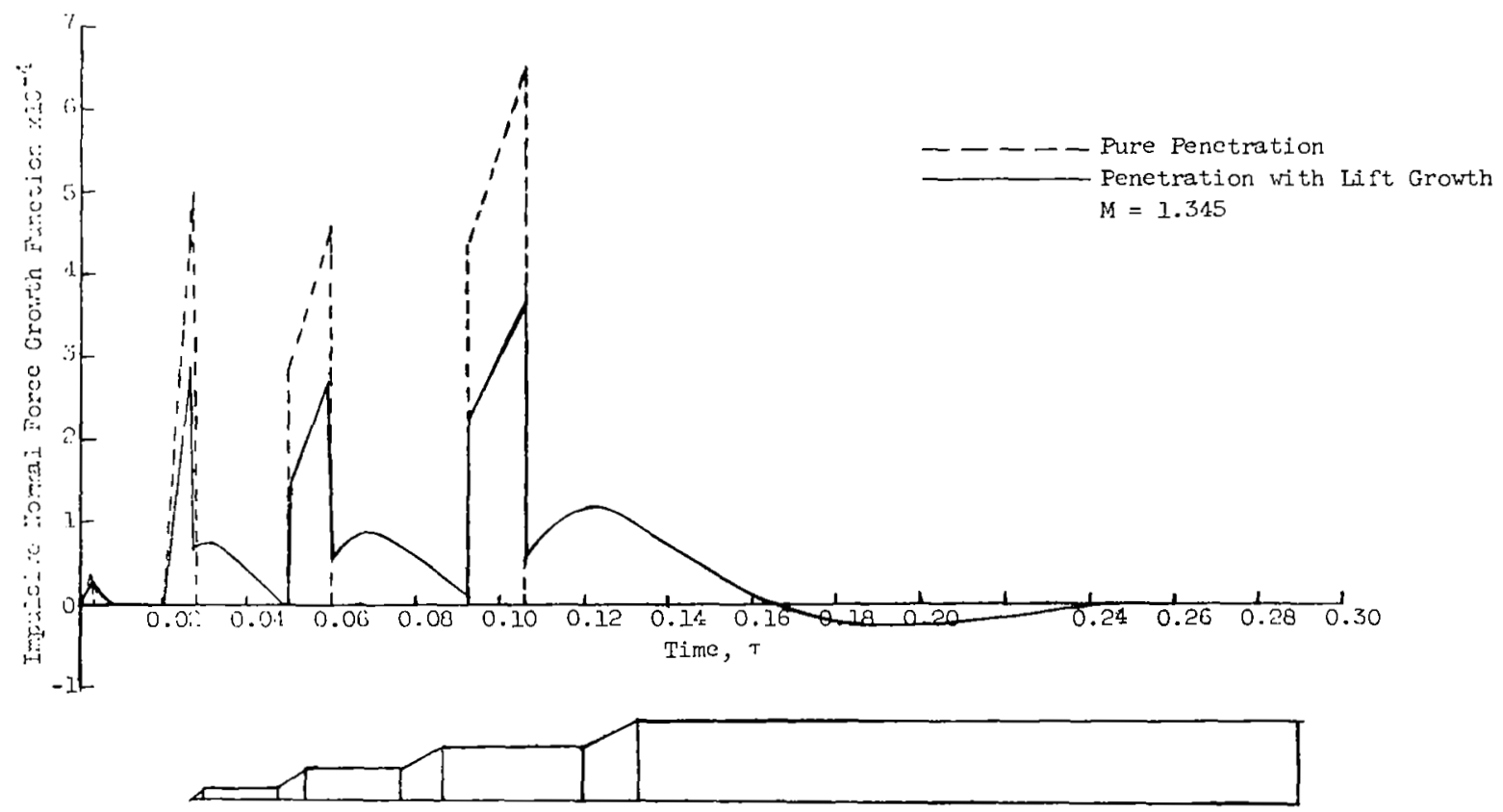

Fig. 4 - Impulsive Normal Force Growth Functions vs. Time in Seconds 

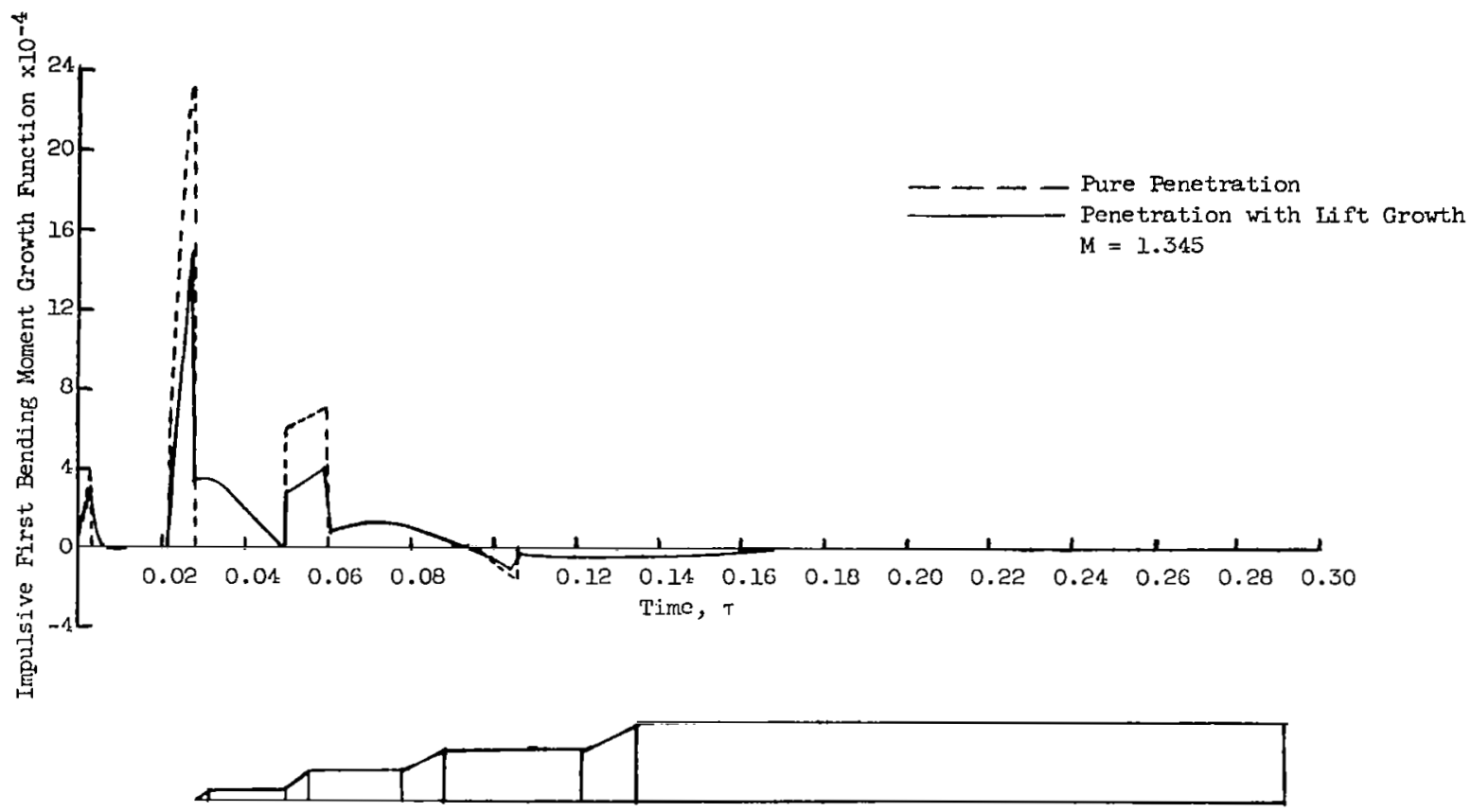

Fic. 5 - Impulsive lst Bending Moment Growth Functions vs. Time in Seconds
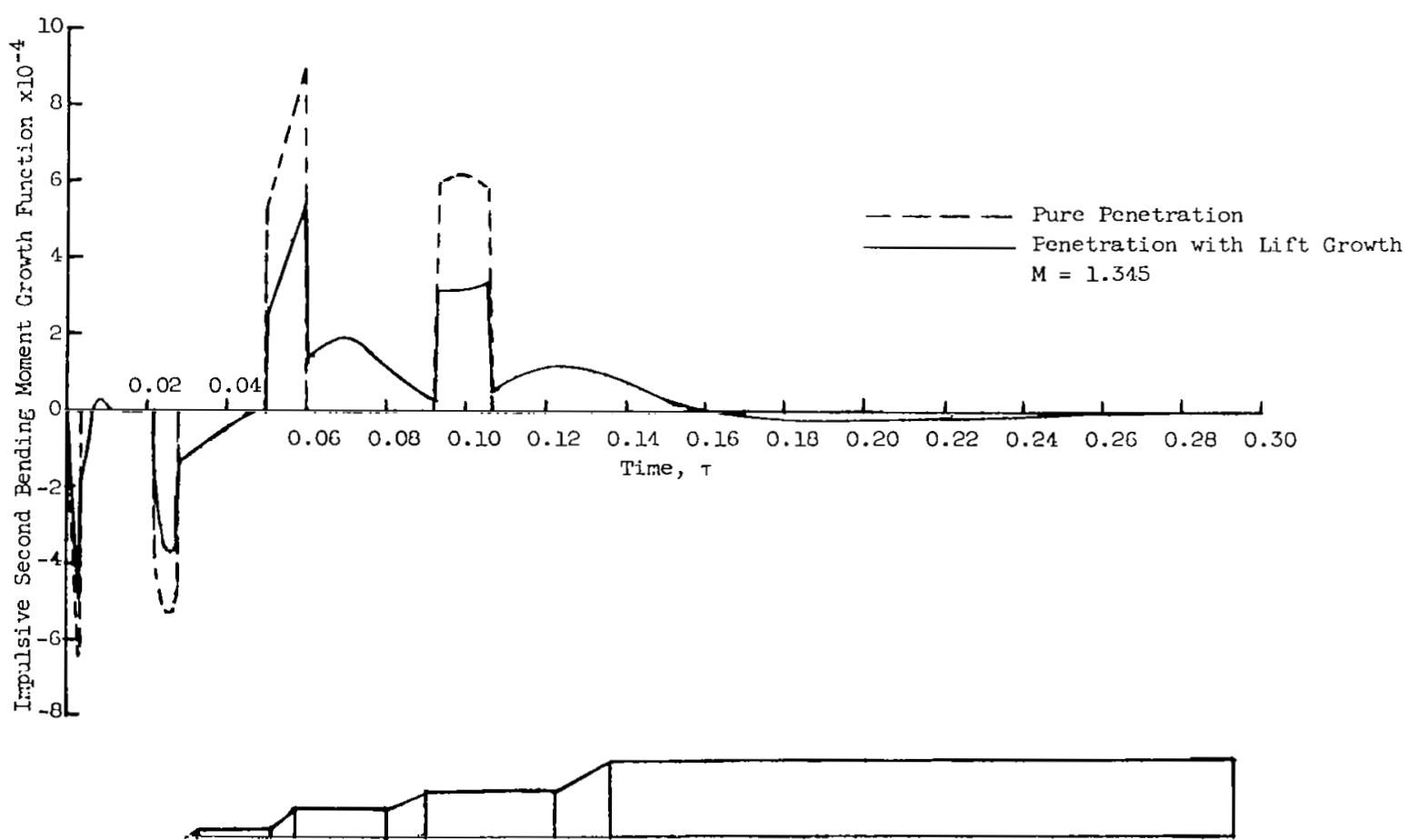

Fig. 6 - Impulsive 2nd Bending Moment Growth Functions vs. Time in Seconds 
The half cross section of the C-5 configuration is given at the bottom of each of the figures for convenience of interpretation of the growth functions. At $\tau=0$, the first region (escape tower) penetrates the gust front and the generalized force buildup begins. The times $\tau=0.0216,0.0500$, and 0.0930 sec. correspond to the successive gust encounters of the second, third and fourth conic regions. The pure penetration and penetration with lift growth curves reached steady-state conditions at $T=0.1065 \mathrm{sec}$. and $T=0.2895 \mathrm{sec}$, respectively. The time for total immersion of the vehicle was $0.2629 \mathrm{sec}$.

For reasons of brevity, a detailed description of the growth functions will not be given in this report. The reader is referred to [1] for some general comments concerning the rigid body growth functions. The general form of the bending moment growth functions are very similar to those of the normal force functions. The bending moment growth functions, however, reflect the character of the mode shapes. The negative growth functions for the fourth region in Fig. 5 and the first and second regions in Fig. 6 correspond to regions of negative displacements of the first and second bending modes, respectively. Figures 2 and 3 reflect an integrated effect of the growth functions in Figs. 5 and 6 , respectively.

\section{Method of Solution for Indicial and Impulsive Responses of Saturn C-5}

The method of Runge-Kutta was used to calculate the impulsive and indicial responses of the Saturn C-5. A fourth order* Runge-Kutta integration equation was used to numerically integrate the equations of motion. Saturn C-5 missile response calculations were obtained for six different sets of aerodynamic environments. These six sets of forcing functions $\{$ see Appendix III, Table VII $\}$ contain the indicial and impulsive aerodynamic forces resulting from

\section{Penetration with lift growth effects, \\ 2. Pure penetration effects, and \\ 3. Instantaneous-immersion effects.}

\footnotetext{
* The Runge-Kutta integration expression used is considered to be of fourthorder accuracy $\{$ see $[3]\}$.
} 
The Runge-Kutta integration process requires knowledge of the initial conditions (or starting values). Since the system of equations used in the investigation can be written as a second order set, \{see (II-II) through (II-19) $\}$, only the initial conditions for the dependent variables and their first derivatives are needed. The initial conditions for each of the six sets of forcing functions were found through use of Laplace transform techniques $\{$ see Appendix III $\}$.

The indicial and impulsive responses of the C-5 were computed at discrete flight times or altitudes from a system of linear, differential equations with constant coefficients* ${ }^{*}$ see (III-1) $\}$. Each set of constant coefficients, and consequently the associated responses, is applicable in a specified time or altitude interval.** A computer program used to obtain the response calculations is discussed in Vol. III.

In each flight time or altitude band, the responses were computed for a real time interval of $20 \mathrm{sec}$. Extremely fine increments of response time were used in computing the forcing functions and the indicial and impulsive responses of the deflections and their first derivatives. Coarser increments were used in the printing and plotting of the output. Only the deflections were plotted on the $\mathrm{SC}-4020$.

Twenty-second response records were computed to insure that the responses had achieved satisfactory steady-state values. For the flight times considered, all of the irmulsive*** responses had converged to 1 per cent or less of their maximum values at the end of $20 \mathrm{sec}$.

The calculation of $20-\mathrm{sec}$. response records does not violate the assumption of constant coefficients as might be expected. From a subsidiary response analysis, it was found that if the sloshing degrees of freedom were removed from the system, the responses of the remaining system (excluding translation) would achieve satisfactory steady-state values within 7 or 8 sec.

* Previous work has shown that it is permissible to use sets of constant coefficients.

** Response calculations were obtained at every $10 \mathrm{sec}$. of flight time between 30 and $100 \mathrm{sec}$. The applicable interval of flight time for each set of these responses is taken as $10 \mathrm{sec}$. Thus, for example, the 30-sec. response calculations (obtalned using the coefficients at 30 sec.) are considered valid between flight times of 25 and $35 \mathrm{sec}$.

***The decay of the indicial responses will be discussed later in this section. 
of response time. The sloshing modes of the C-5 are very slightly damped in comparison to the other wodes of the system (except translation). Therefore, since we are considering a coupled system, the response calculations for rotation, first and second bending and control after 7 or 8 sec. reflect the influence of the sloshing modes. This influence is basically governed by that part of the equation of motion which contains time invariant coefficients.

The selection of the increment size used in the numerical calculation of the responses was dictated by two requirements:

1. The increment size should be sufficiently small as to permit an accurate calculation of the aerodynamic forces.

2. The increment size should permit 32 calculated response values per cycle for the highest frequency component of the system.

Based on the above requirements, the increment sizes, $\Delta T$, used in the response calculations are presented in Table $I$.

TABLE I

TABULATION OF INCREMENT SIZES USED IN INDICIAL

AND IMFULSIVE RESPONSE CALCULATIONS

\begin{tabular}{cccccc}
$\begin{array}{c}\text { Flight Time } \\
(\mathrm{sec} .)\end{array}$ & $\begin{array}{c}\Delta \tau_{1} \\
(\mathrm{sec})\end{array}$ & $\begin{array}{c}\tau_{1} \\
(\mathrm{sec})\end{array}$ & $\begin{array}{c}\Delta \tau_{2} \\
(\mathrm{sec})\end{array}$ & $\begin{array}{c}\tau_{2} \\
(\mathrm{sec})\end{array}$ & $\begin{array}{c}\Delta \tau_{3} \\
(\mathrm{sec})\end{array}$ \\
40 & 0.0005 & 0.02 & 0.001 & 0.75 & 0.004 \\
50 & 0.0005 & 0.02 & 0.001 & 0.60 & 0.004 \\
60 & 0.0005 & 0.02 & 0.001 & 0.50 & 0.004 \\
70 & 0.0005 & 0.02 & 0.001 & 0.45 & 0.003 \\
80 & 0.0005 & 0.03 & 0.001 & 0.40 & 0.003 \\
90 & 0.0005 & 0.03 & 0.001 & 0.40 & 0.004 \\
100 & 0.0005 & 0.04 & 0.001 & 0.35 & 0.004 \\
\hline & 0.0005 & 0.05 & 0.001 & 0.35 & 0.004
\end{tabular}


The response times, $\tau$, at which the increment size was changed* are also given. $\Delta \tau_{1}$ was used in the calculations from 0 to $\tau_{1} ; \Delta \tau_{2}$ was used from $\tau_{1}$ to $\tau_{2}$; and $\Delta \tau_{3}$ was used from $\tau_{2}$ to the end of the response calculations.

Extremely small increments $\left(\Delta T_{1}=0.0005 \mathrm{sec}.\right)$ are used at the beginning of the response calculations to adequately describe the high-frequency components of the control system. The $T_{1}$ values correspond to the times at which these frequency components are negligible. The $T_{2}$ values correspond to conservative estimates of the times when the aerodynamic forcing functions reach steady-state conditions.

Since a voluminous amount of indicial and impulsive response data were generated, only a representative quantity of these data is presented. Plots of the indicial and impulsive responses of first bending, $\eta_{I}$, second bending, $T_{2}$, and control deflection, $\beta_{C}$ (noted by first control) are given in Figs. 7 through 24 for a flight time of $70 \mathrm{sec}$. The impulsive responses are presented in Figs. 7 through 15; the indicial responses are given in Figs. 16 through 24. For each coordinate, the first figure reflects the effect of instantaneous immersion; the second figure reflects the effect of pure penetration; and the third reflects the effect of penetration with lift growth.

The numerics presented alongside the plots in each figure pertain to an analysis** of the zero crossings, maximum and minimum values of the respective coordinate response. The format of the information presented is as follows:

$\begin{array}{cc}\text { Response } & \text { Response } \\ \text { Time } & \text { Value }\end{array}$

Response

Maximum, Minimum or

Crossing

The maximum, minimum and zero crossing values are the calculated points which precede (in time) the event.

* It was found expedient, from the standpoint of conserving computer running time, to increase the increment size, when permissible, during the response calculations.

** The write-up on the computer program used to analyze the indicial and impulsive responses is presented in Vol. III. 


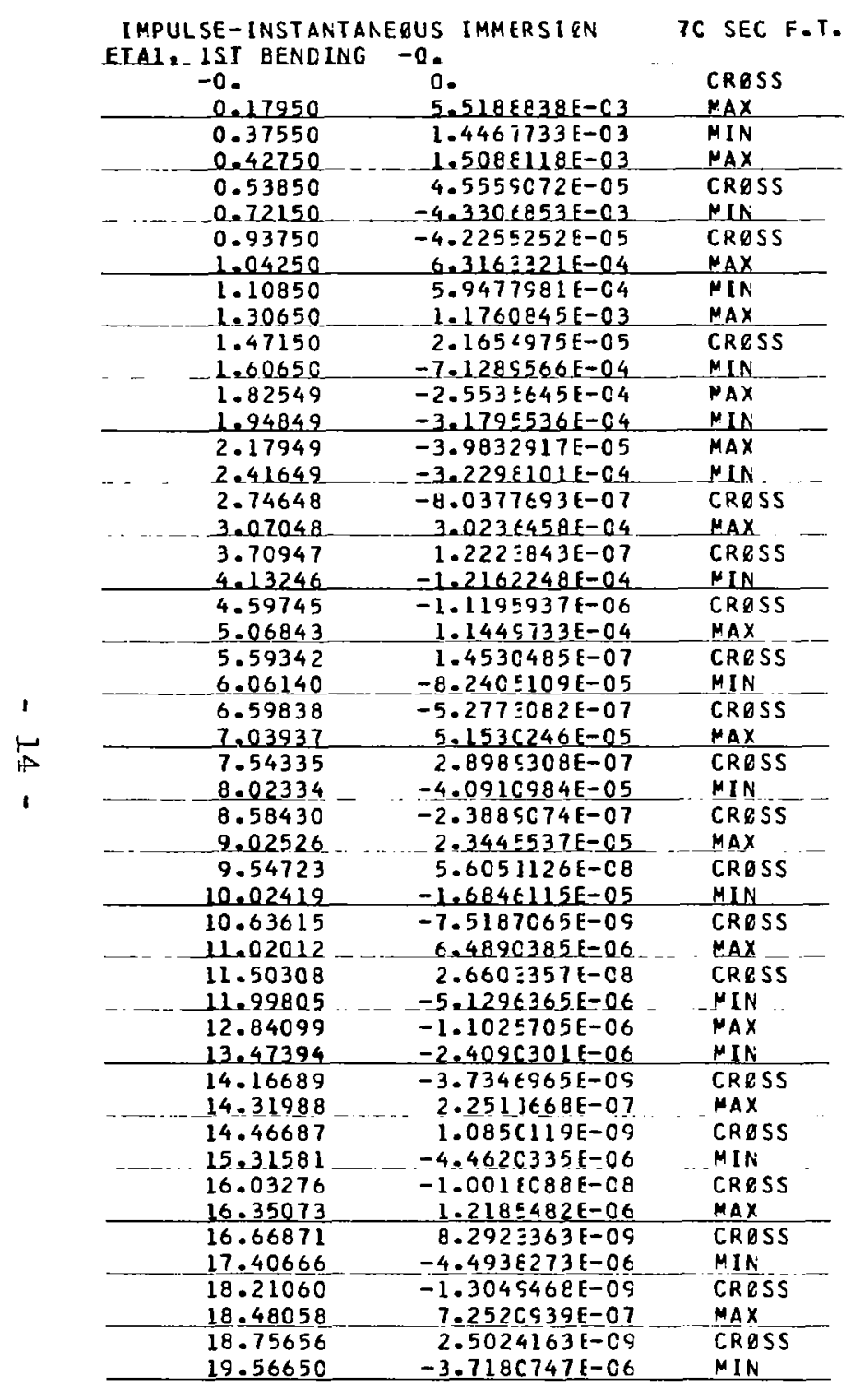

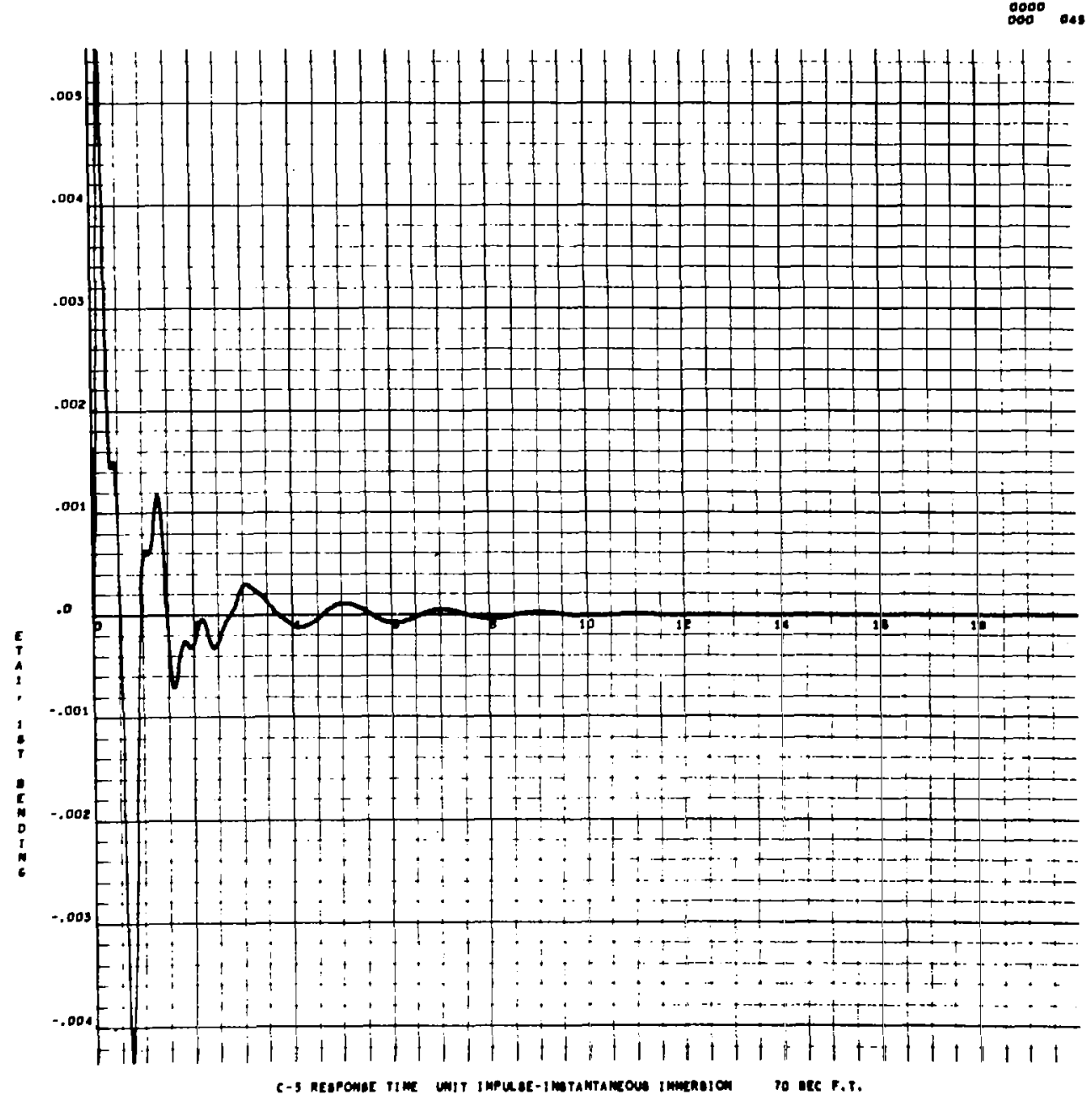

Fig. 7 - Impulsive Response of lst Bending Considering Instantaneous Immersion - 70 sec. F.T. 


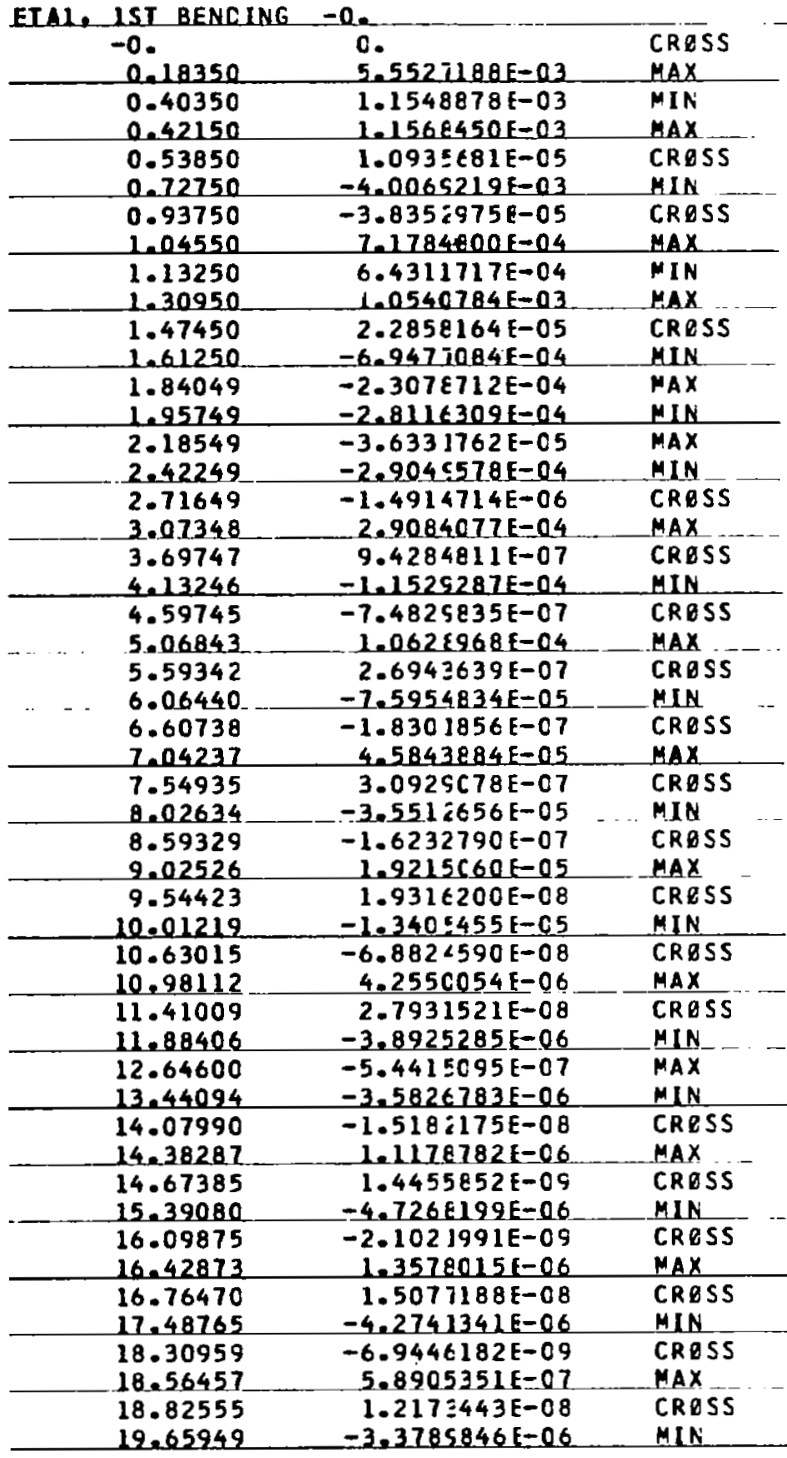

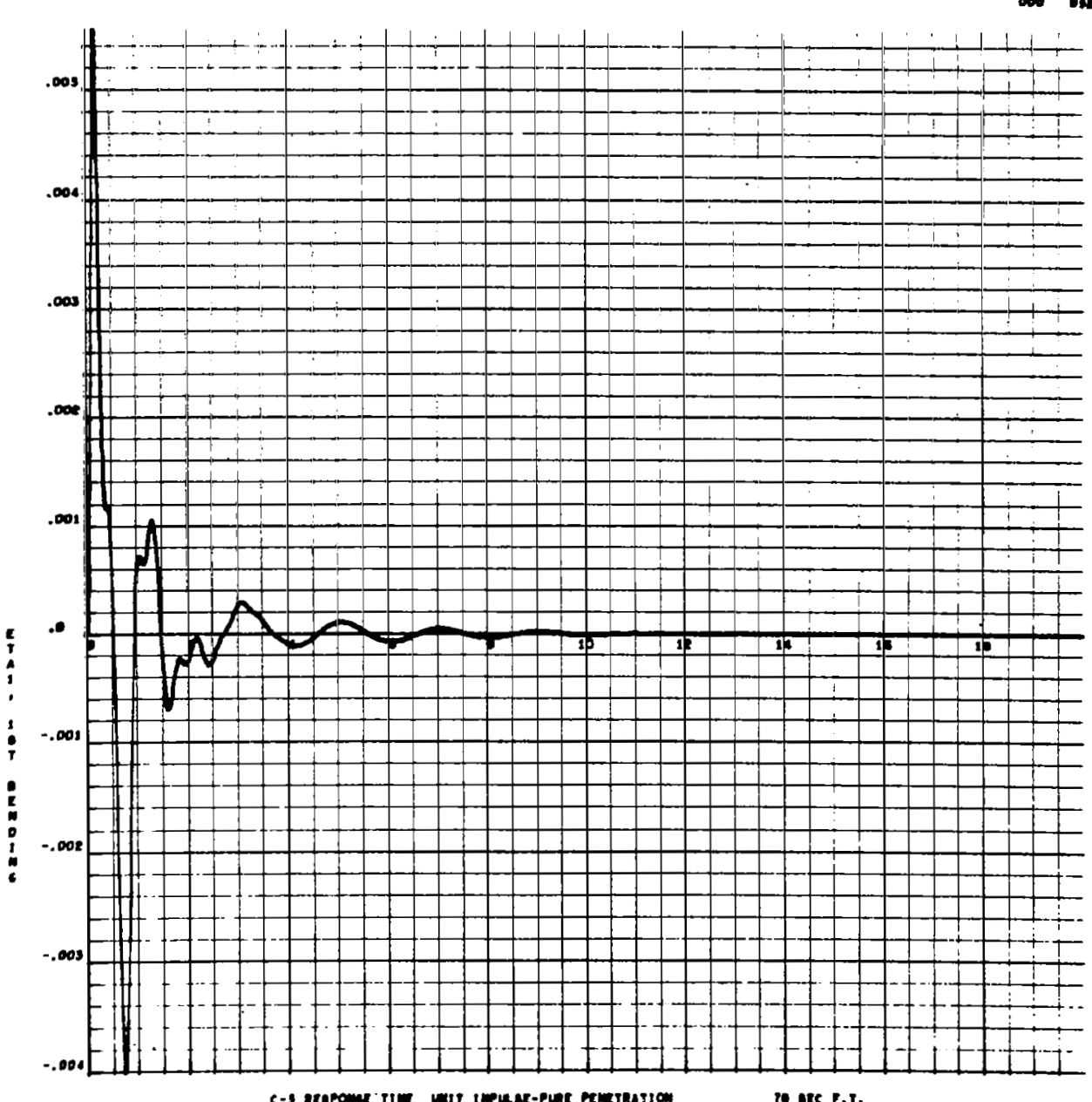

Fig. 8 - Impulsive Response of lst Bending Considering Pure Penetration - 70 sec. F.T. 


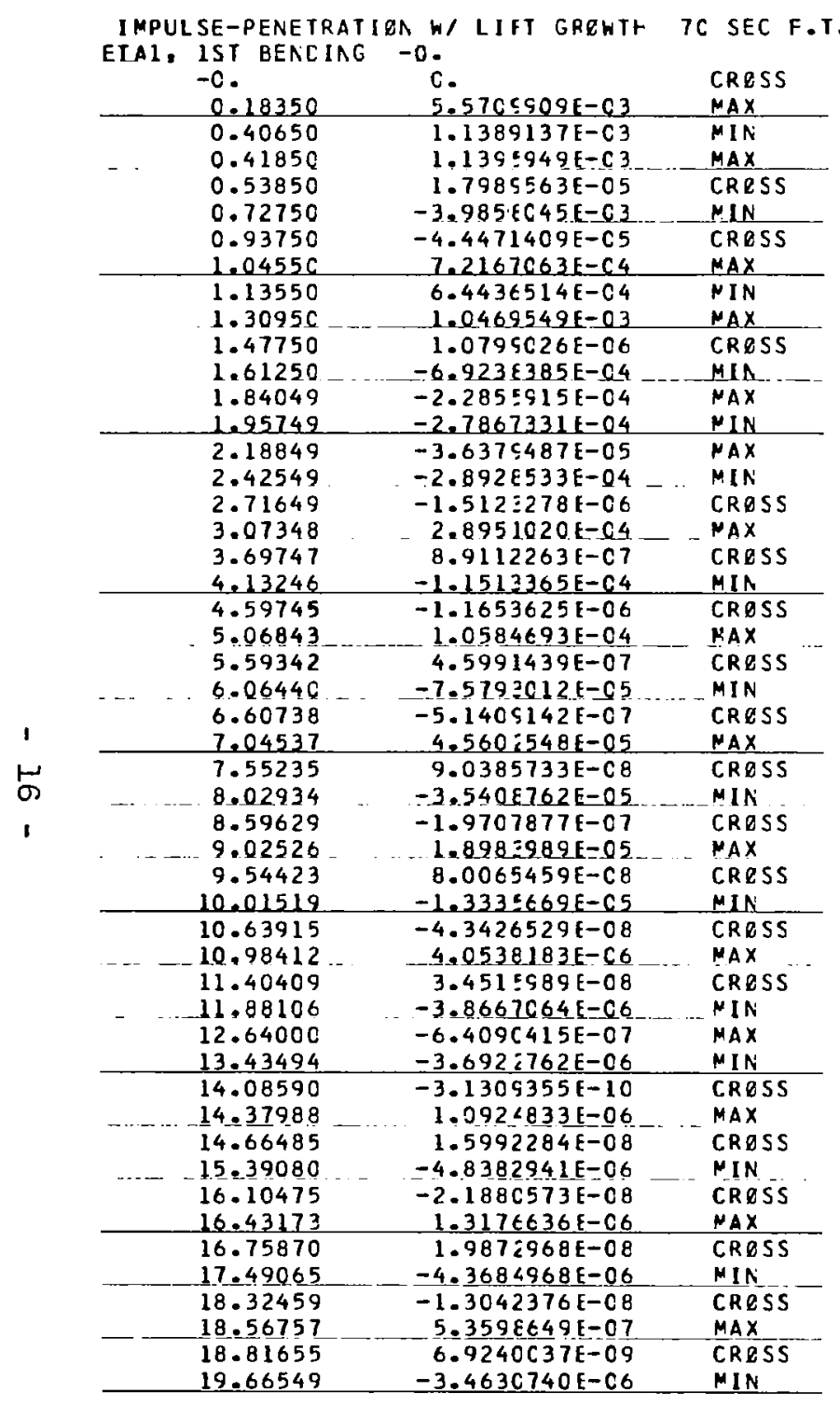

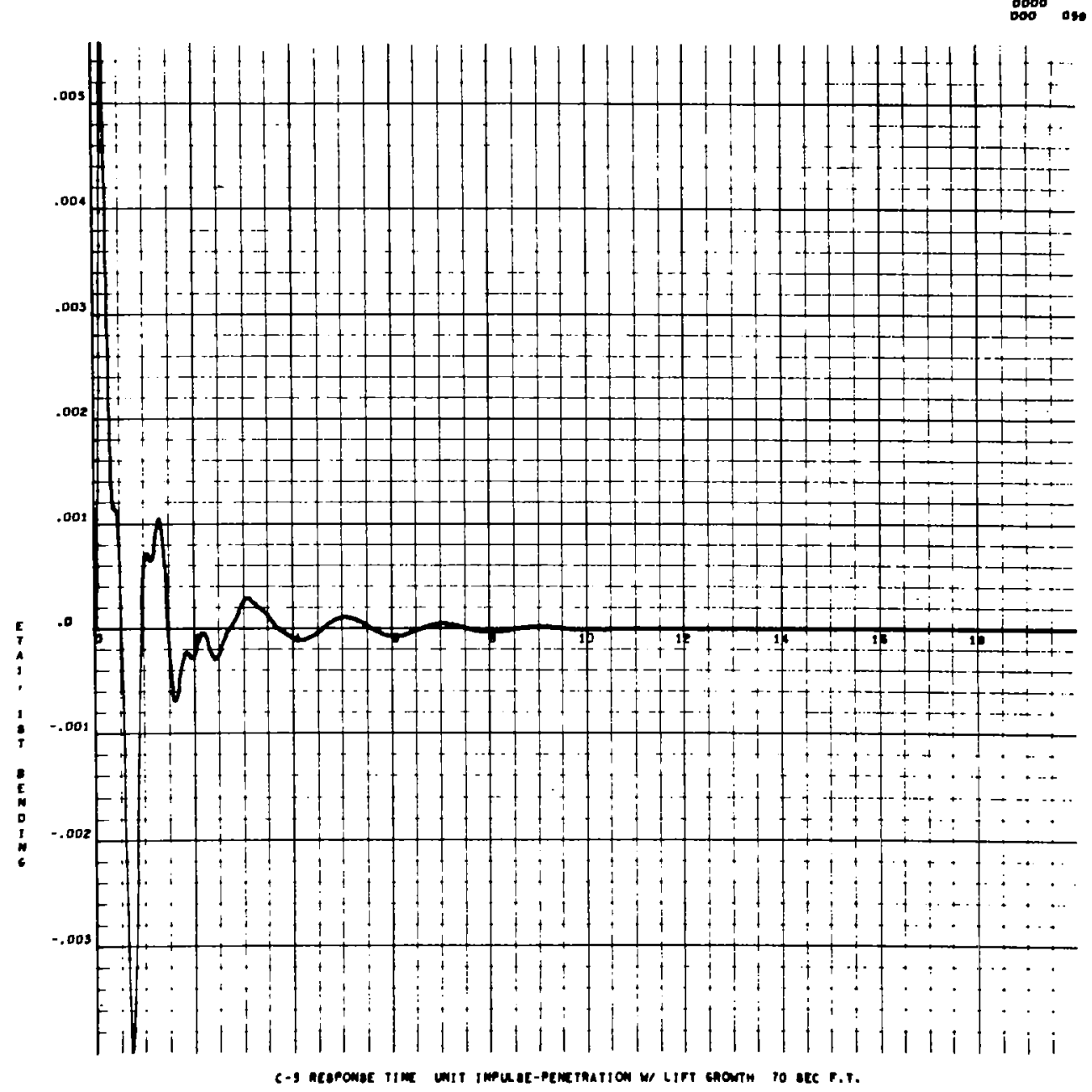

Fig. 9 - Impulsive Response of Ist Bending Considering Penetration with Lift Growth - 70 sec. F.T. 
-0 .

$0.24050 \quad 3.4001262 E-03$

$0.33150 \quad-3.4813480 E-05$ CRZSS

$0.43050-1.358 C 451 E-C 4 \quad$ NIN

$0.5355 \mathrm{C} \quad 2.9217769 \mathrm{E}-03$ MAX

$0.64950 \quad 8.5975 C 62 \mathrm{E}-05$ CRESS

$\begin{array}{lll}0.75750 & -2.267 C C 43 E-03 & \text { NIN } \\ 0.88650 & -2.6112218 \mathrm{E}-05 & \text { CRQSS }\end{array}$

$0.97050 \quad 9.2321383 \mathrm{E}-04 \quad$ FAX

$1.06350 \quad 1.8501940 E-05$ CRQSS

$1.16550 .-8.8081745 \mathrm{E}-04$ MIN

$1.27050 \quad-1.0457354 E-05$ CRESS

$\begin{array}{lll}1.37850 & 8.0816466 \mathrm{E}-04 & \text { MAX } \\ 1.50150 & 4.8960832 \mathrm{E}-\mathrm{C6} & \text { CRESS }\end{array}$

$1.60050 \quad-4.8848773 \mathrm{E}-04$ NIN

$1.72350 \quad-1.1126033$ [-05 CRRS

$1.79849 \quad 1.8355871 \mathrm{E}-\mathrm{C} 4$ MAX

$1.87949 \quad 1.0831702 \mathrm{E}-06$ CROSS

$\frac{2.00249}{2.14049}-\frac{-3.2985864 E-04}{\text { NIN }}$

$2.22449 \quad 1.2748119 E-04$ MAX

$2.32049 \quad 2.963 ! 439 \mathrm{E}-06$ CRES

$2.42849 \quad-1.3052271 \mathrm{E}-\mathrm{C} 4 \quad$ NIN

$2.54849-1.1642305[-C 6$ CRESS

$2.64749 \quad 8.0176153 \mathrm{E}-05$ MAX

$2.77048 \quad 1.8371378 \mathrm{E}-06$ CRESS

$2.83348 \quad-2.3493297 E-C 5$ NIN

$2.89648 \quad-2.9478158 \mathrm{E}-07$ CRESS

$3.0644 \mathrm{R} \quad 1.0397987 \mathrm{E}-04$ MAX

$\begin{array}{lll}3.28648 & 2.7937388 E-C 5 & \text { MIN } \\ 3.44248 & 5.0035046 E-C 5 & \text { MAX }\end{array}$

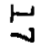

,

\begin{tabular}{|c|c|c|}
\hline 3.44248 & $5.0035046 E-0$ & MAX \\
\hline 3.61347 & $6.0097505 \mathrm{~F}-07$ & CRESS \\
\hline 3.72447 & $-1.967 \div 556 E-05$ & \\
\hline 3.87447 & $-9.9644 E 53 E-C 6$ & $\max$ \\
\hline $\begin{array}{l}4.09647 \\
4.53745\end{array}$ & $\begin{array}{l}-3.0571266 \mathrm{E}-05 \\
-9.5508525 \mathrm{E}-0 \mathrm{~S}\end{array}$ & $\begin{array}{l}\text { PIA } \\
\text { CRESS }\end{array}$ \\
\hline 5.06843 & $2.6594633 E=05$ & MAX \\
\hline 5.52742 & $1.588 \sqsubseteq 557 f-c 8$ & CRESS \\
\hline 5.99840 & $-1.963 E 817 E-05$ & NIN \\
\hline 6.55039 & $-1.0178026 \mathrm{E}-07$ & CRESS \\
\hline 6.98537 & $1.1961005 \mathrm{E}-05$ & MAX \\
\hline 7.49535 & $5.2307922 \mathrm{E}-\mathrm{CB}$ & CRASS \\
\hline 7.98434 & $-9.7621507 E-06$ & min \\
\hline 8.57230 & $-1.6778188 \mathrm{E}-\mathrm{C} 8$ & CRESS \\
\hline 9.01326 & $5.299 \equiv 718 E-06$ & FaX \\
\hline 9.55023 & $1.424 C 013 E-C 8$ & CROSS \\
\hline 10.04219 & $-3.613 \subseteq 44 C E-C 6$ & MIA \\
\hline 10.73514 & $-4.7717727 E-09$ & CROSS \\
\hline 11.09811 & $0.1845755 \mathrm{E}-07$ & MAX \\
\hline 11.60508 & $4.530<221 \mathrm{E}-09$ & CRESS \\
\hline 12.99098 & $-1.0706983 \mathrm{E}-\mathrm{C6}$ & MIN \\
\hline 13.75292 & $-1.697 C 801 E-09$ & CRESS \\
\hline 14.12189 & $4.3658570 \mathrm{E}-07$ & $\operatorname{MAX}$ \\
\hline 14.46387 & $3.9309<16 E-09$ & CRESS \\
\hline 15.19882 & $-1.560 i 308 E-C 6$ & MIN \\
\hline 15.90076 & $-4.0836504 E-09$ & CRESS \\
\hline 16.27574 & $5.672 \leq 248 E-07$ & $\operatorname{maX}$ \\
\hline .6537 & $4.0096487 E-09$ & CRESS \\
\hline
\end{tabular}

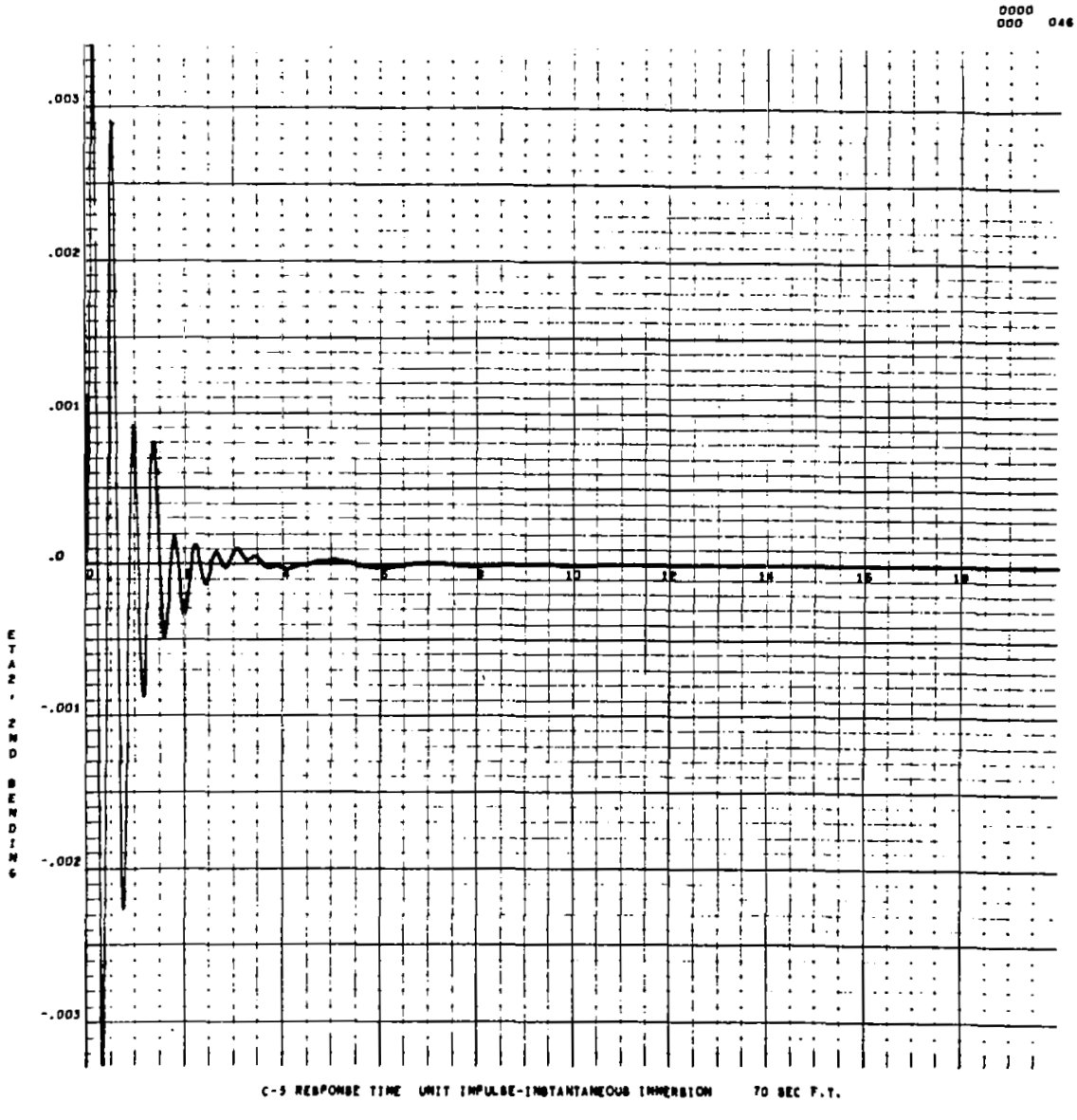

Fig. 10 - Impulsive Response of 2nd Bending Considering Instantaneous Immersion - 70 sec. F.T. 

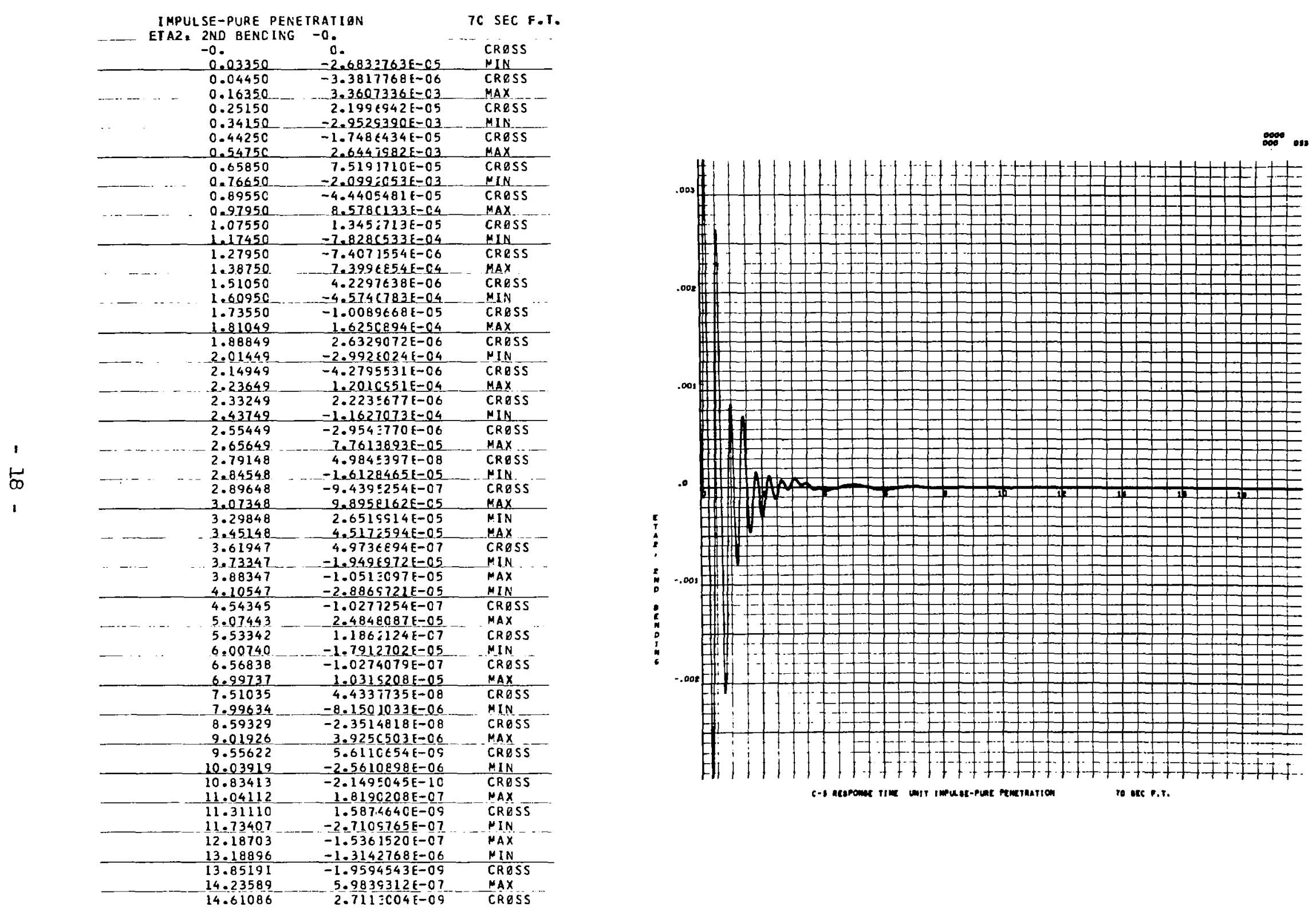

Fig. 11 - Impulsive Response of 2nd Bending Considering Pure Penetration - 70 sec. F.T. 


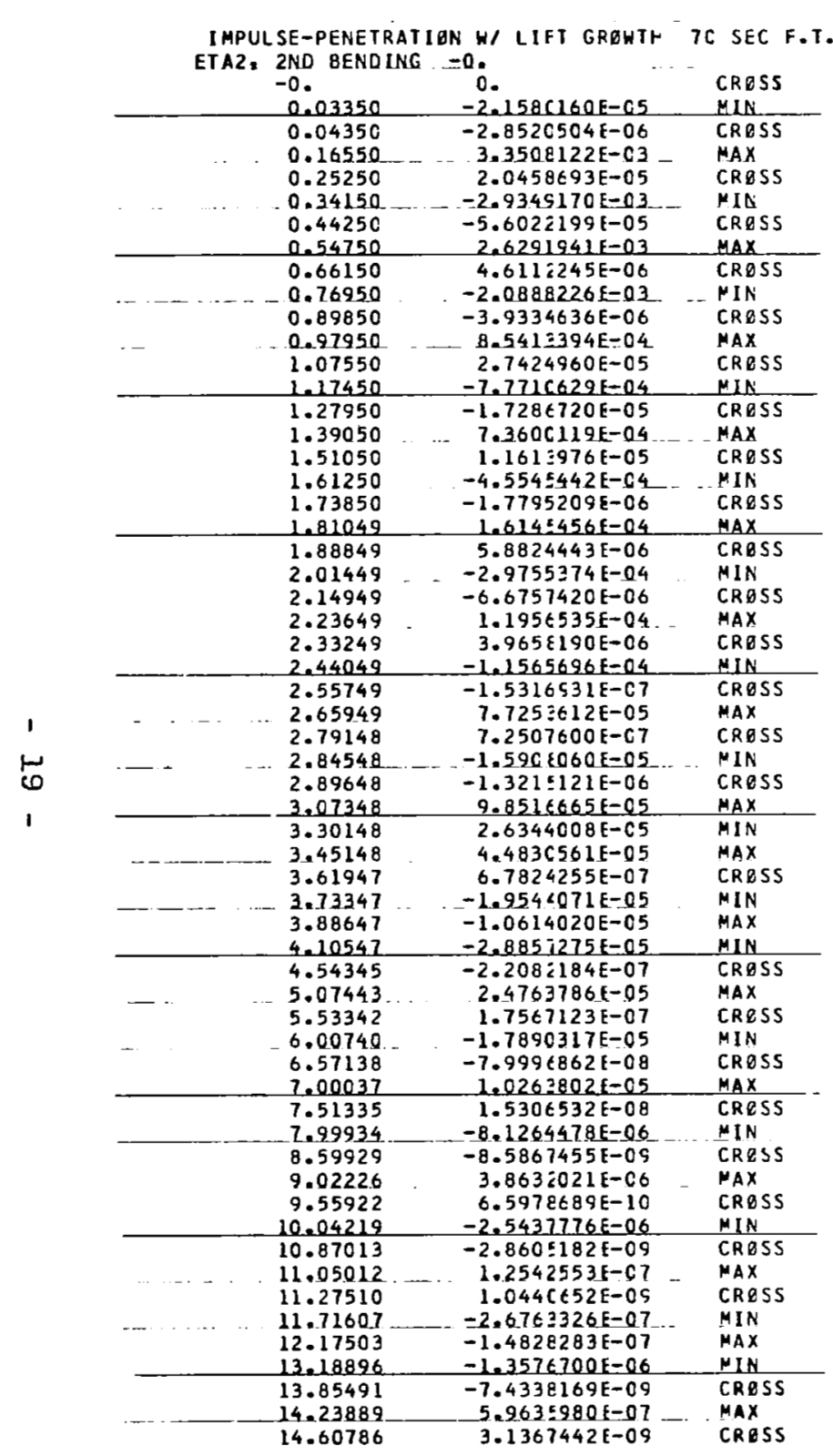

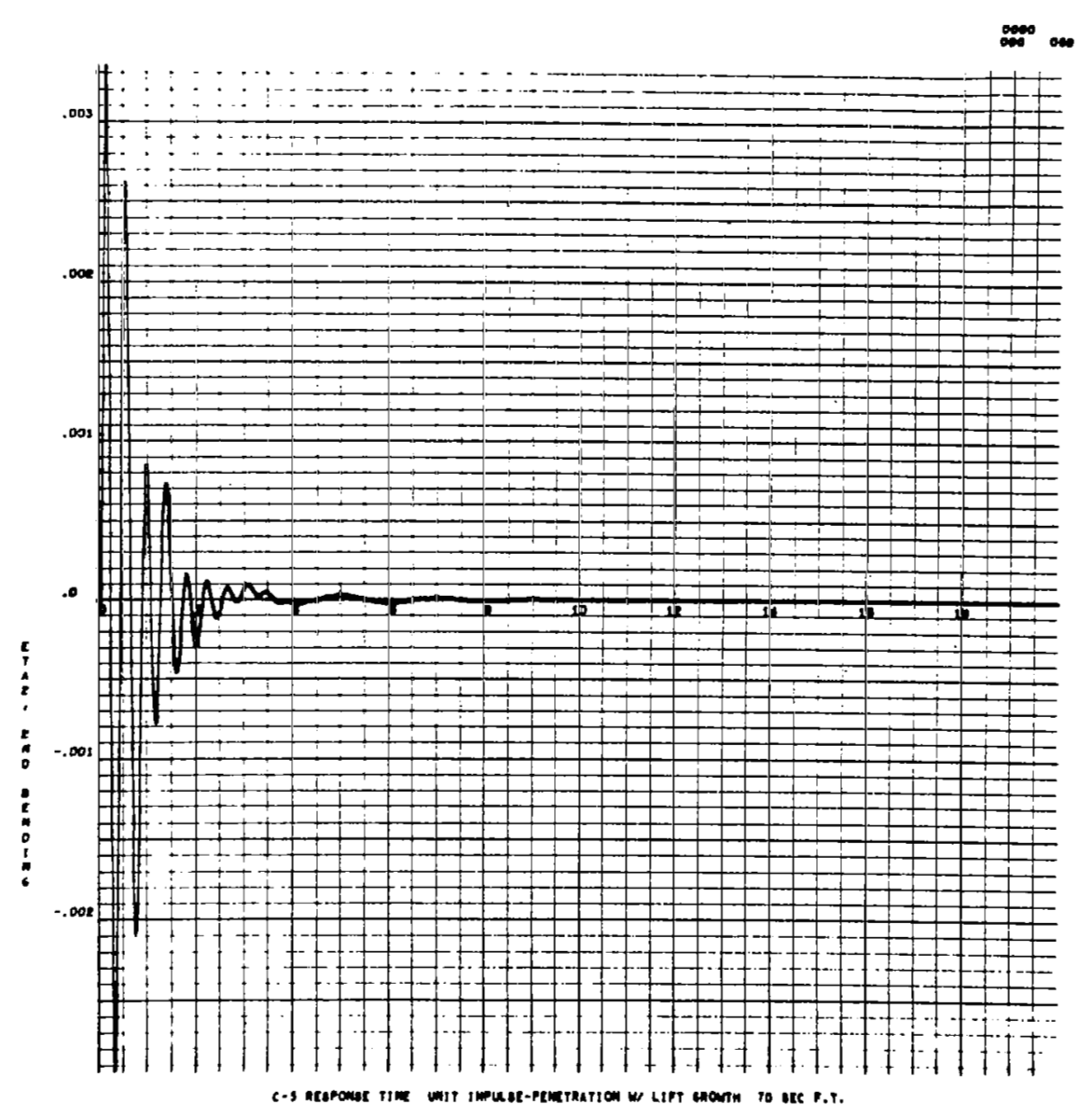

Fig. 12 - Impulsive Response of and Bending Considering Penetration with Lift Growth - 70 sec. F.T. 


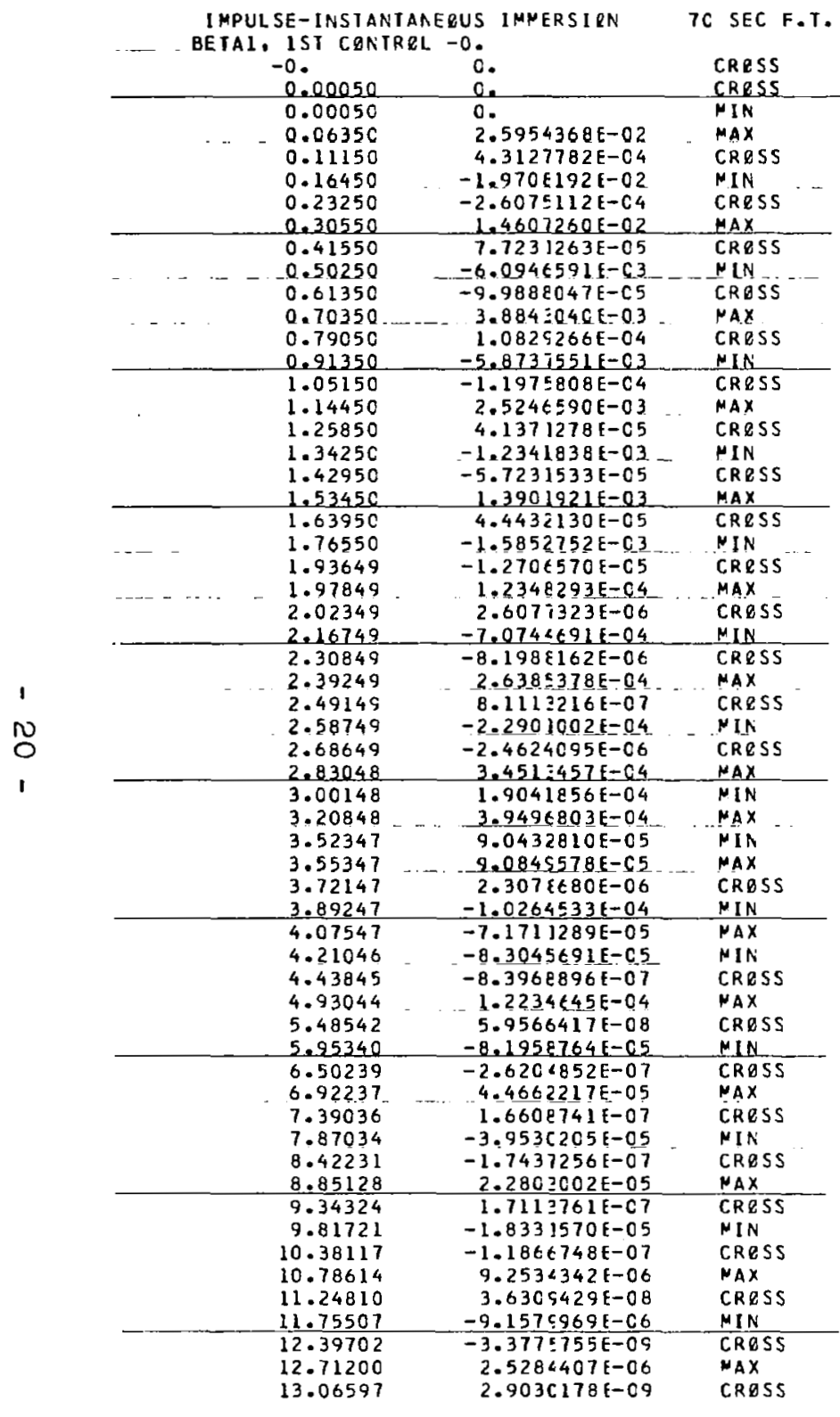

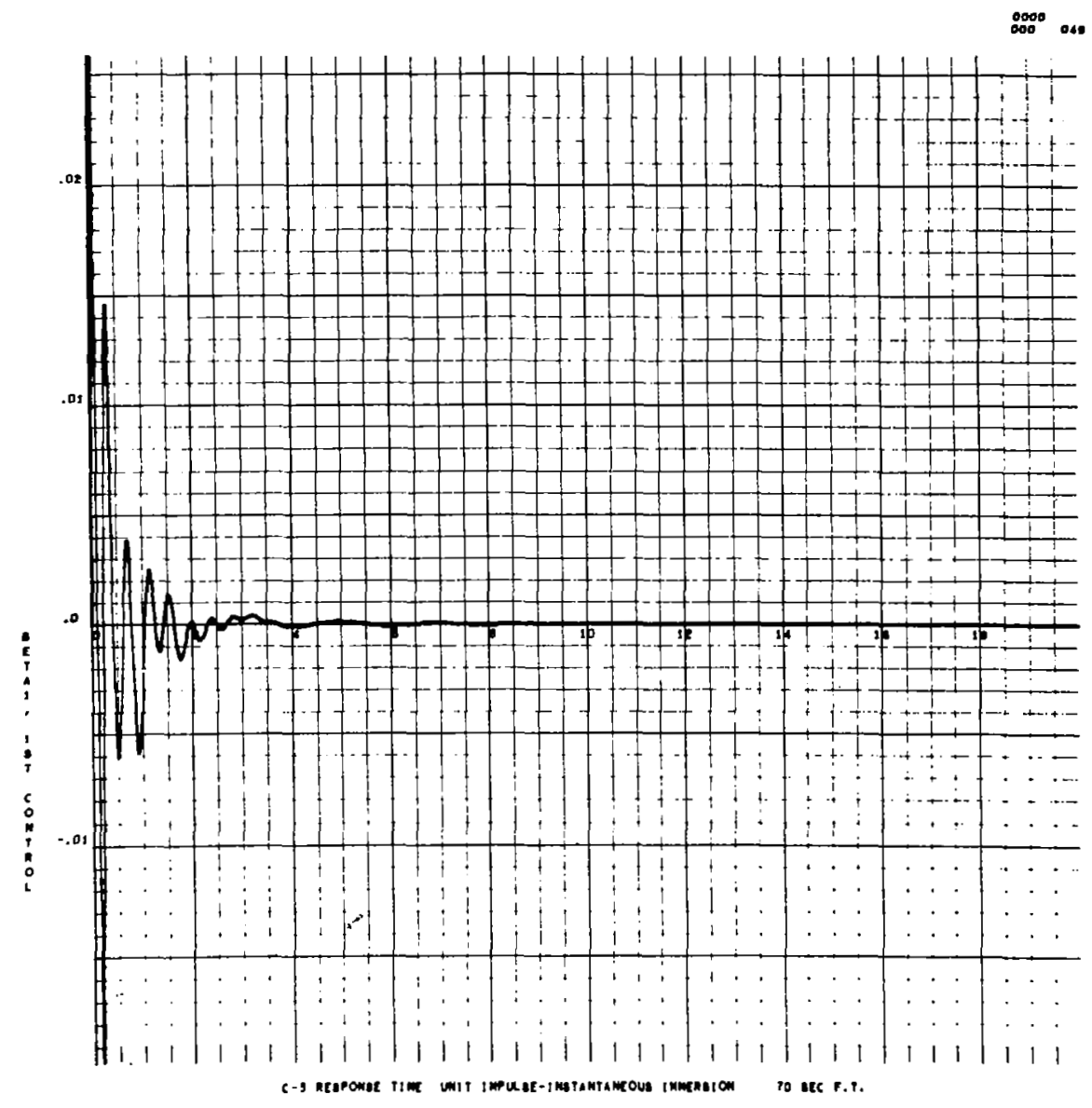

Fig. 13 - Impulsive Response of lst Control Considering Instantaneous Immersion - 70 sec. F.T. 


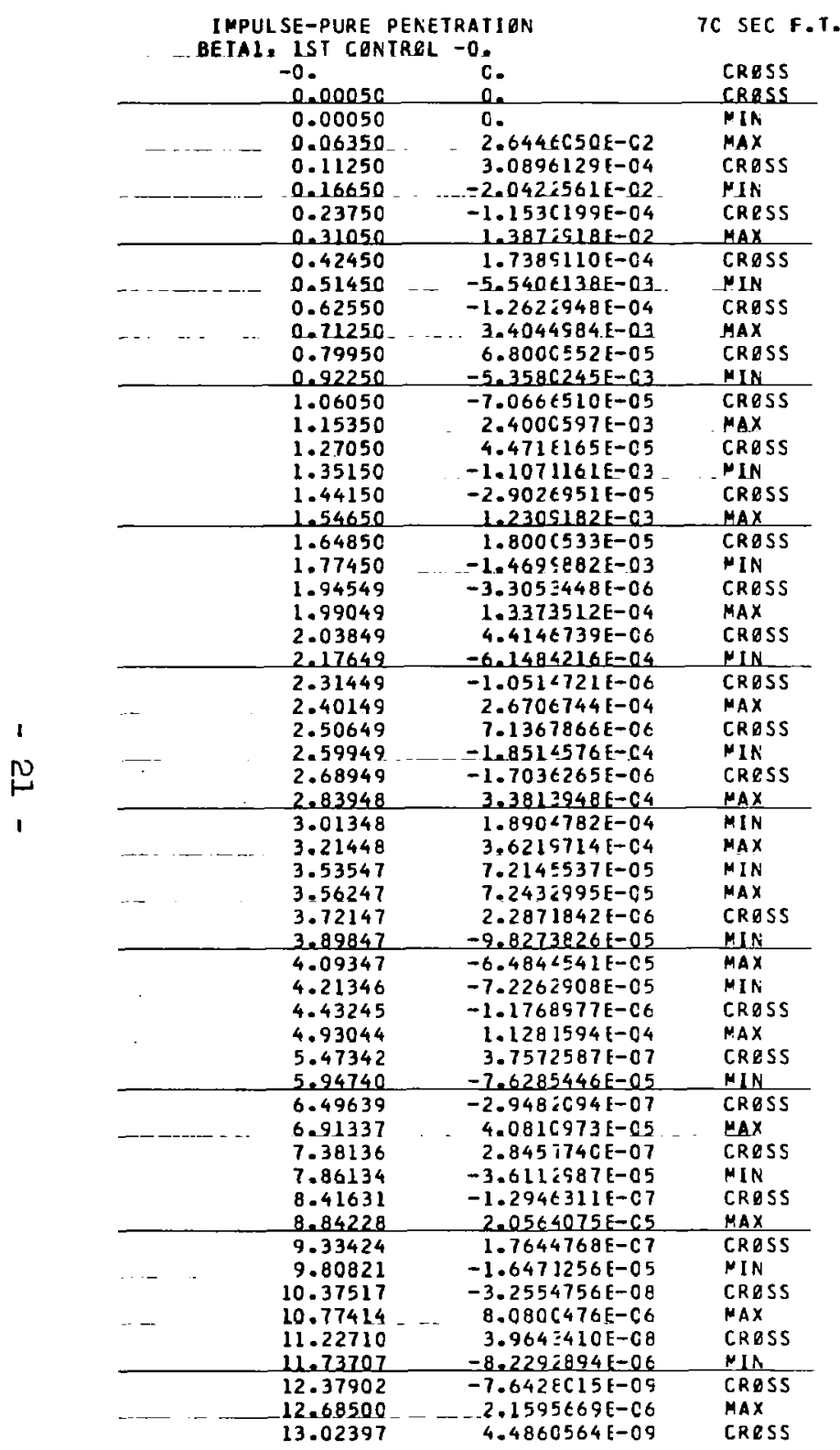

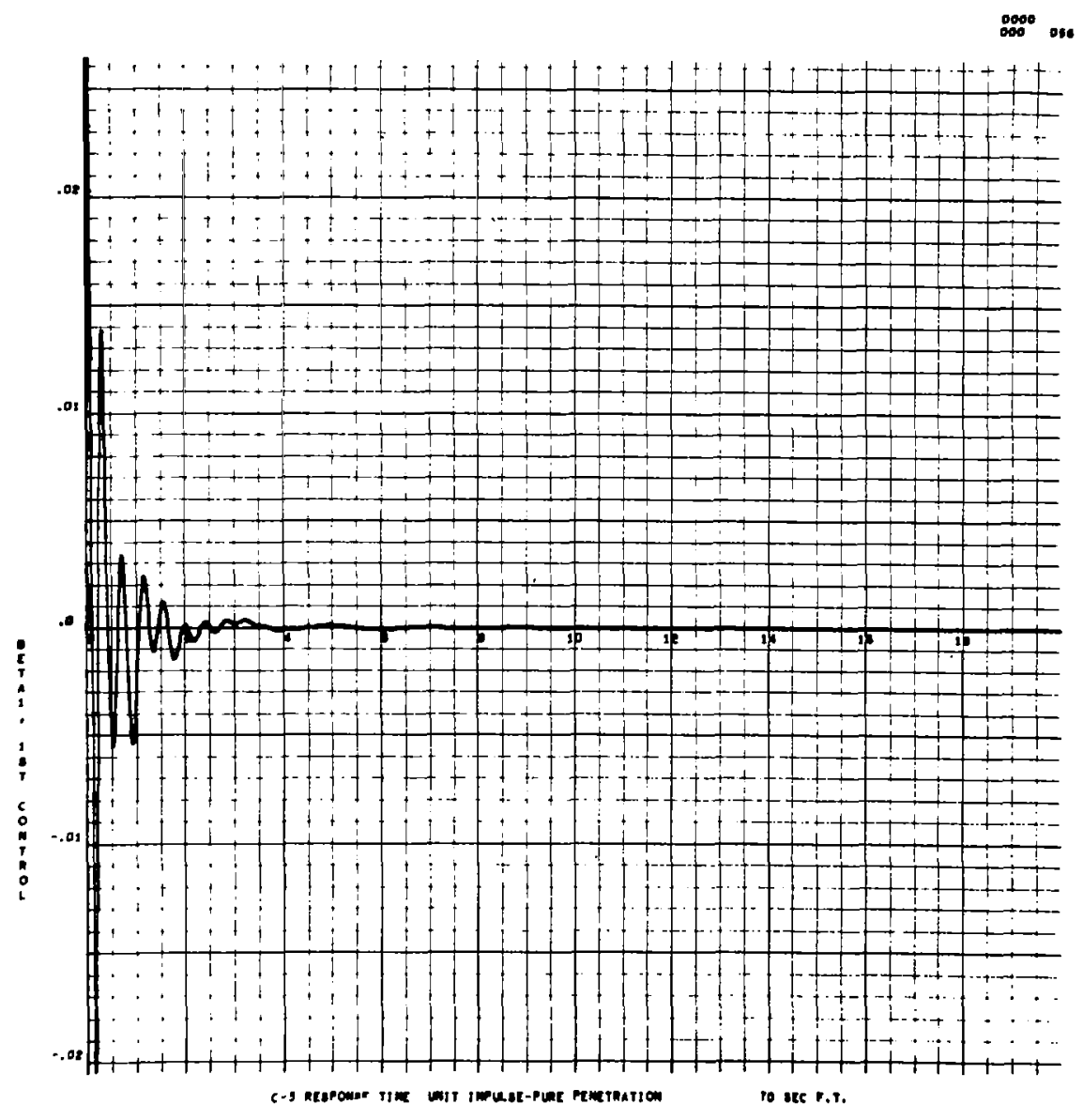

Fig. 14 - Impulsive Response of lst Control Considering Pure Penetration - 70 sec. F.T. 
IMPULSE-PENETRATION W/ LIFI GRQHTH TC SEC F.T.

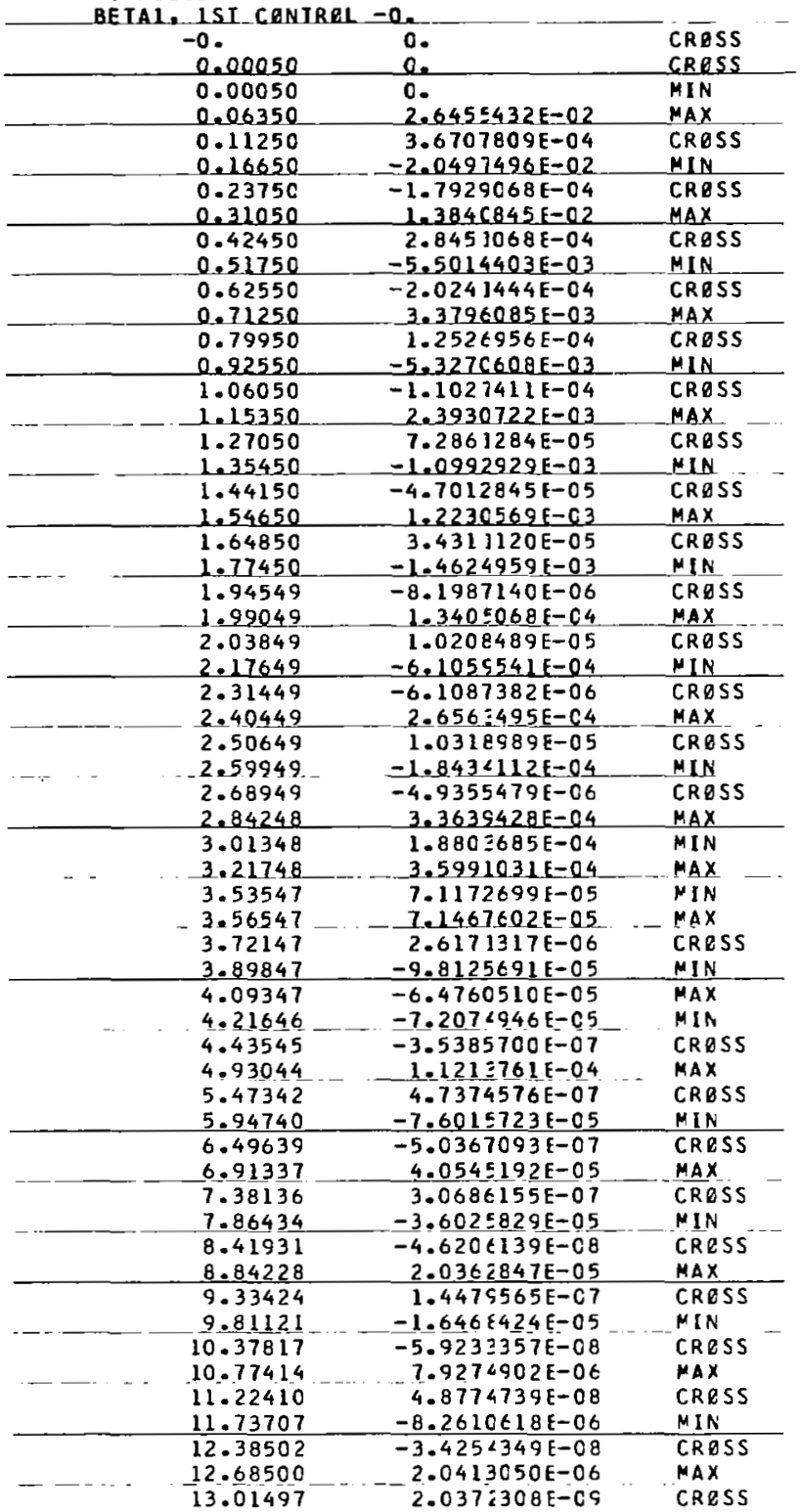

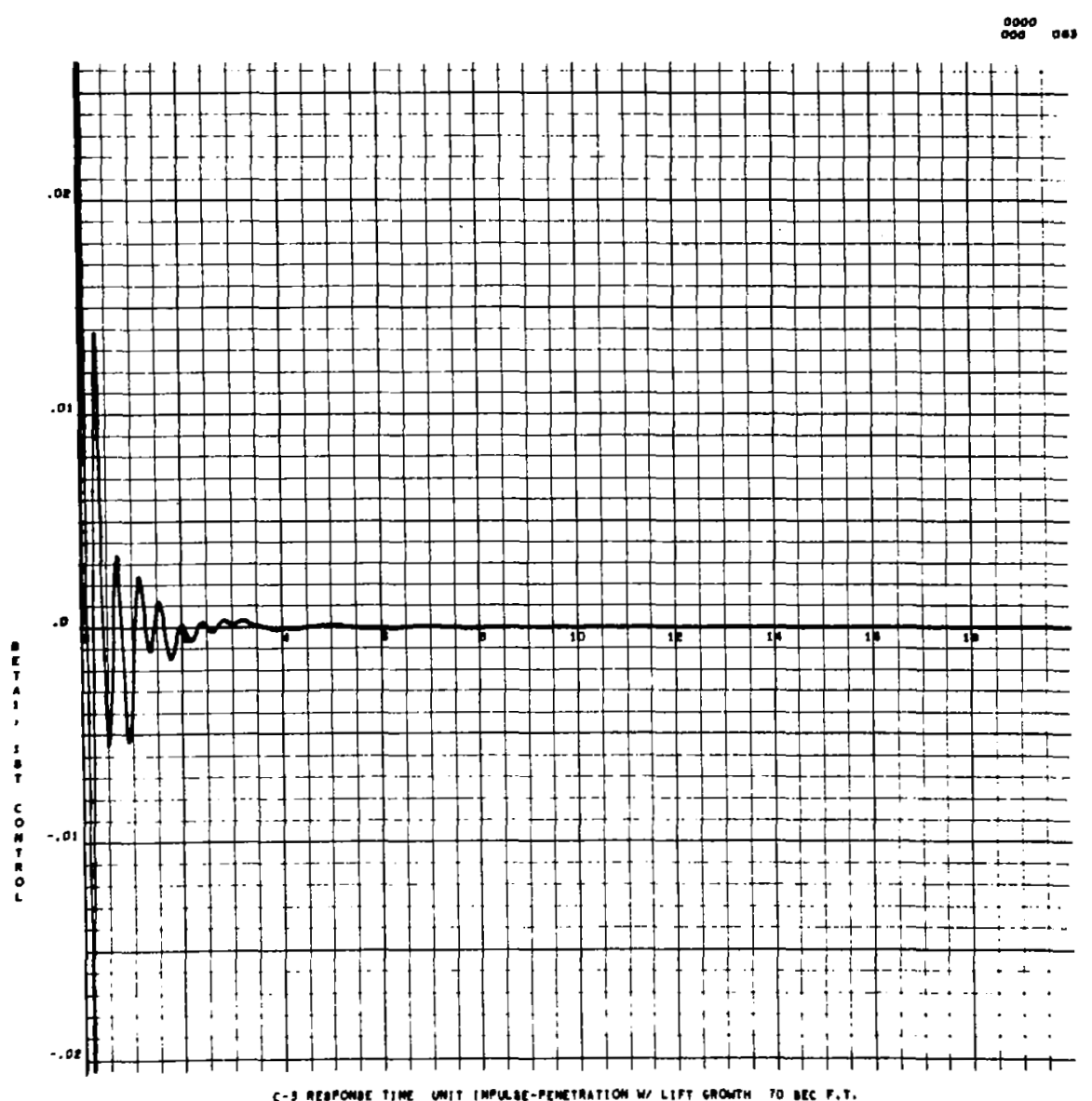

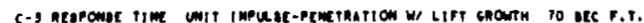

Fig. 15 - Impulsive Response of lst Control Considering Penetration with Lift Growth - 70 sec. F.T. 
STEP -INSTANTANEEUS IMMERSIEN

TC SEC F.T.

ETA1. 1ST BENC ING . - - O.

2814551E-C3 CRQSS

0.54150 1.281t551E-C3

$0.94050 \quad 2.7695439 \mathrm{E}-04$

1.47450 6.5851603E-C4

$3.70947 \ldots \quad 4.8553897 \mathrm{E}-04 \ldots$ MAX

$4.60045 \quad 4.1927464 \mathrm{E}-\mathrm{C} 4 \quad$ MIN

$5.59342 \quad 4.912 i 708 \mathrm{~F}-04 \quad \mathrm{MAX}$

$\begin{array}{lll}6.60138 & 4.386 \zeta 508 E-04 & \text { MIN }\end{array}$

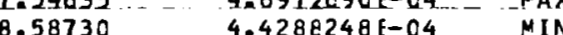

$9.54723 \quad 4.572 \mathrm{C} 323 \mathrm{E}-04$

$9.54723-4.45753933 \mathrm{E}-\mathrm{C} 4$. MAX

$\begin{array}{lll}10.63615 & 4.4578933 E-C 4 & \text { MIN } \\ 11.50308 & 4.4938123 \mathrm{E}-\mathrm{C} 4 & \text { MAX }\end{array}$

$14.16689 \quad 4.4311240 \mathrm{E}-04 \quad$ MAX

$14.16989-\quad 4.4311240 \mathrm{E}-04 \quad$ MIN

$14.46687-4.4315708 \mathrm{E}-\mathrm{C4}$ MAX

$-16.03276 \quad-4.39128785-04$ MIA

$16.66871 \quad 4.3963691 E-04$ MAX

$18.21060 \quad 4.3552158 E-04$ MIN

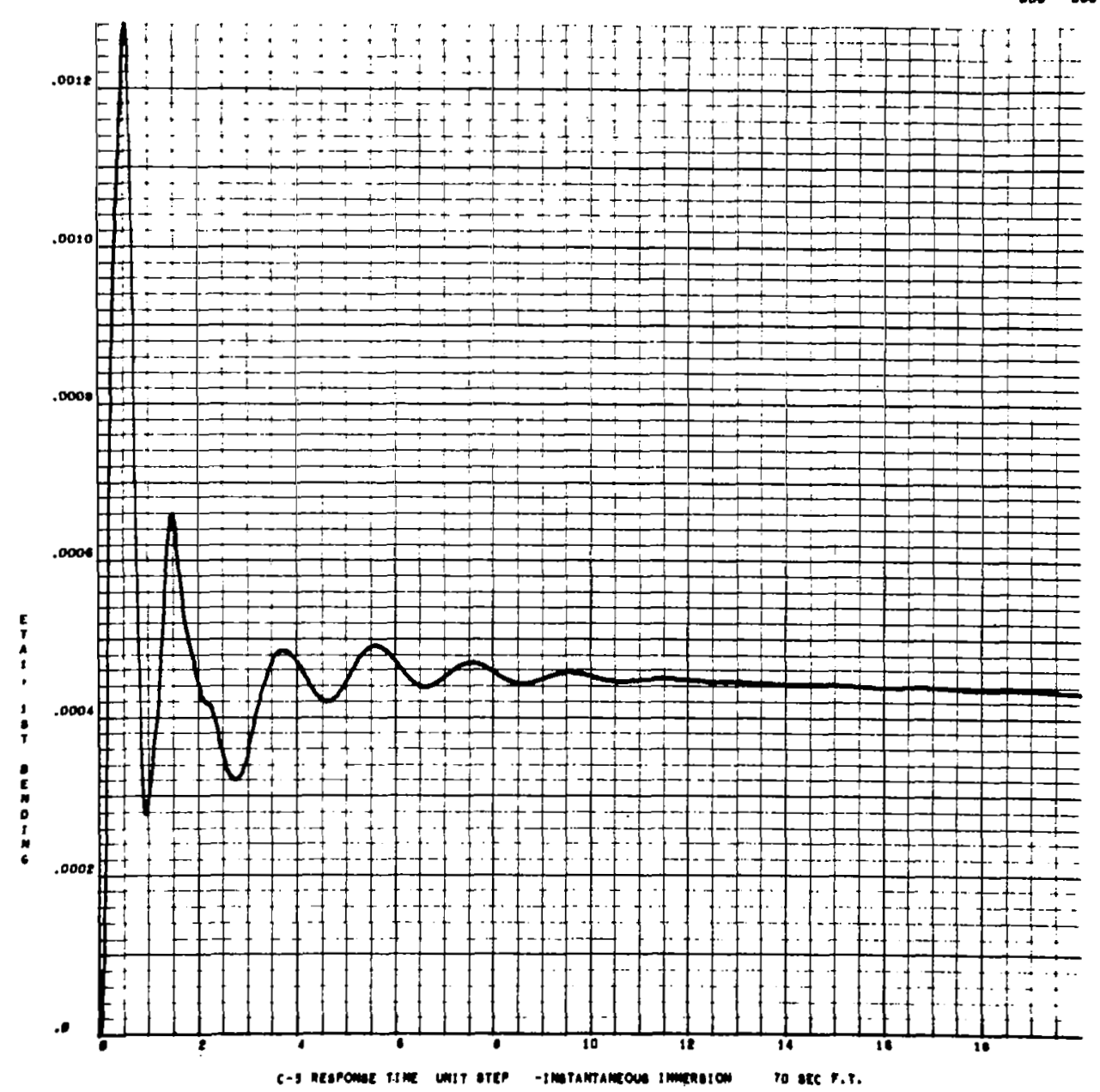

Fig. 16 - Indicial Response of Ist Bending Considering Instantaneous Immersion - 70 sec. F.T. 


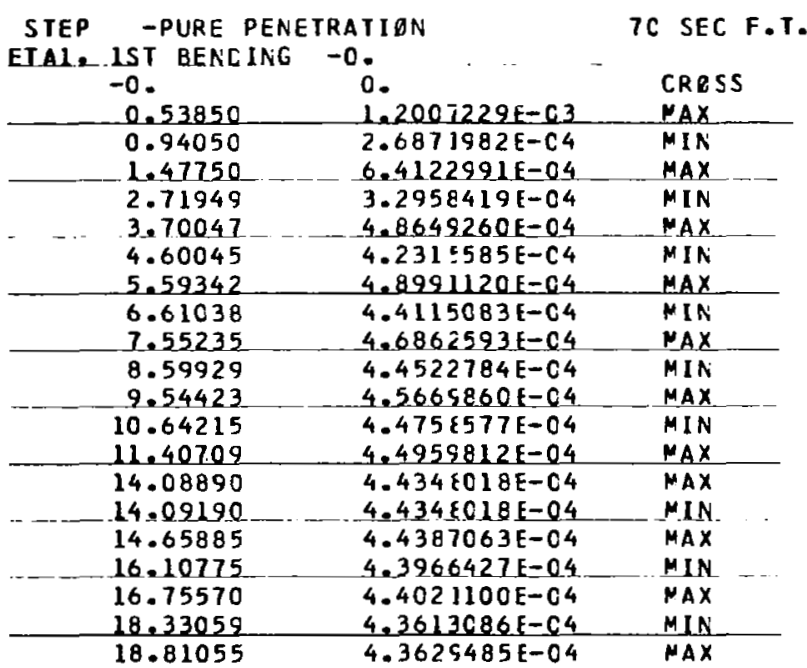

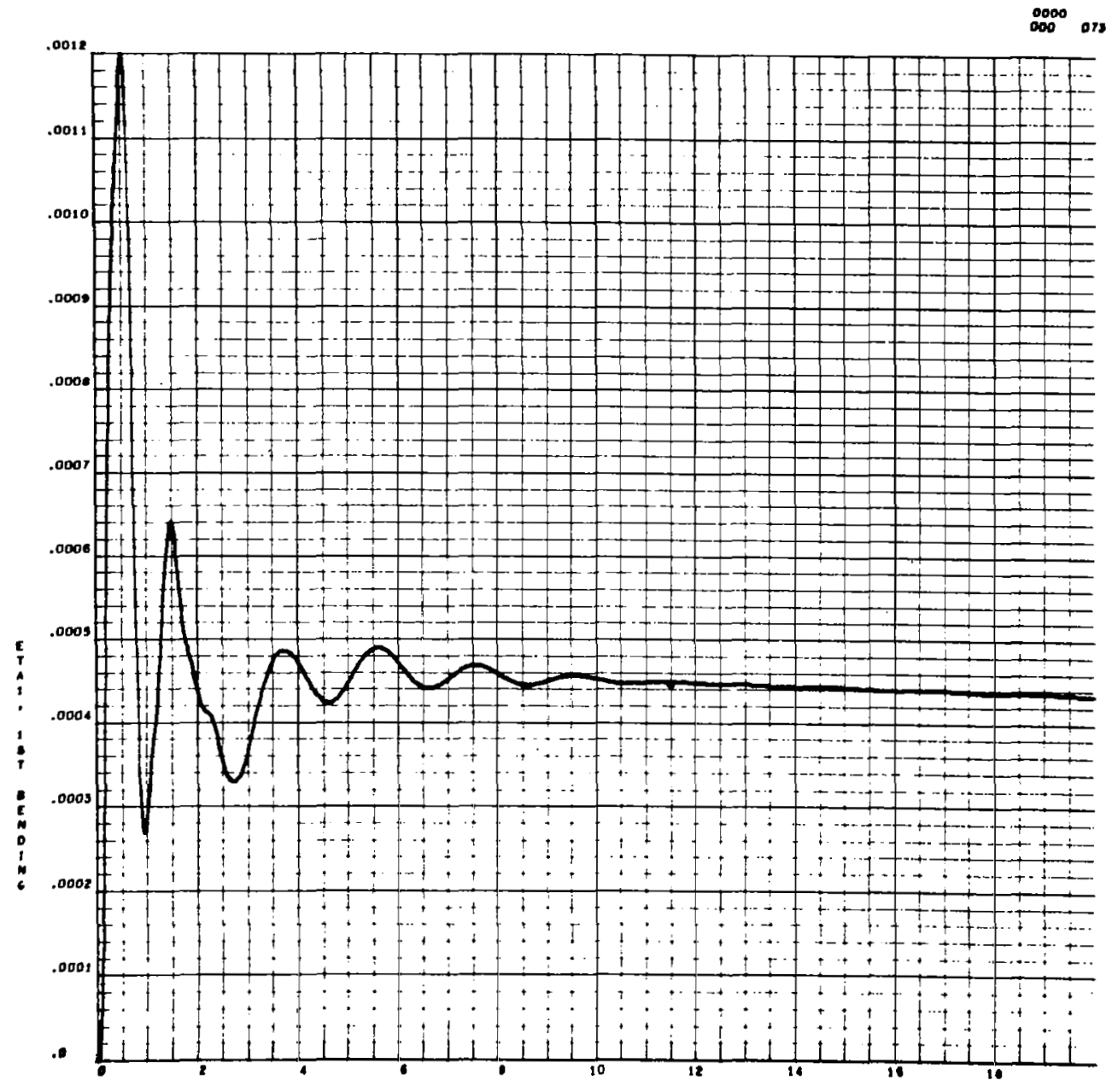

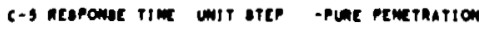

o aec e.t.

Fig. 17 - Indicial Response of lst Bending Considering Pure Penetration - 70 sec. F.T. 
-STEP -PENETRATION W/ LIFT GFOWTH TC SEC F.T.

EIAL. ISI BENCING _- $Q$.

-0 .

0.53850

0.

\begin{tabular}{ccc}
0.53850 & $1.1976214 \mathrm{~F}-03$ & MAX \\
\hline 0.94050 & $2.6885107 \mathrm{E}-04$ & MIN \\
1.47750 & $6.4043538 \mathrm{E}-04$ & MAX
\end{tabular}

$1.47250-6.4043538 \mathrm{E}-04 \quad \mathrm{MAX}$

$2.71949 \quad 3.2987538-C 4 \quad M I N$

$\begin{array}{rrr}3.70041 & 4.865 C 416 \mathrm{E}-04 \\ 4.60045 & 4.232\{296 \mathrm{E} \rightarrow 04 & \text { MAX } \\ \text { MIN }\end{array}$

$5.59642 \quad 4.8987313 \mathrm{~F}-04 \quad$ MAX

$6.61038 \quad 4.412: 880 E-C 4 \quad M I N$

7.55235 - $4.6855940 \mathrm{E}=04 \ldots \mathrm{MAX}$

$8.59929 \quad 4.453 C 283 \mathrm{E}-\mathrm{C4} \quad \mathrm{MIN}$

$9.54723--4.5668750 E-04-M A X$

10.64215 MIN

$11.40409-4.4960770 \mathrm{~F}-04 \quad \mathrm{MAX}$

11.40709
$14.08890 \quad 4.496 C 770 E-04$

$14.66485-4.439 \mathrm{C} 379 \mathrm{E}-\mathrm{C4}$ - MAX

$16.11075-4.3968502 \mathrm{E}-04-\mathrm{MIN}$

$16.75870 \quad 4.4023768 \mathrm{f}-\mathrm{C4}$ MAX

$18.33359-4.3615359 \mathrm{E}-04 \quad$ MIA

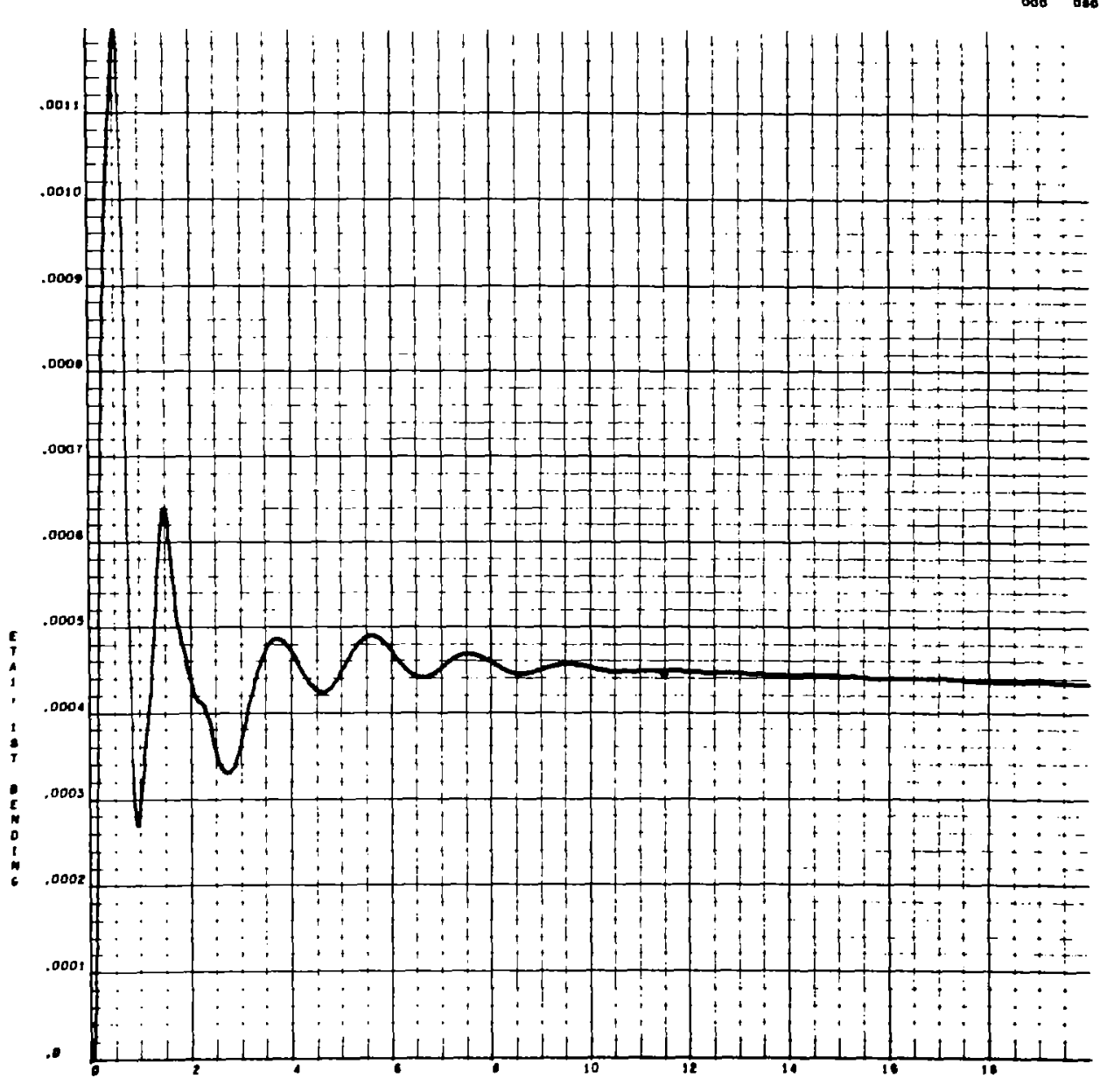

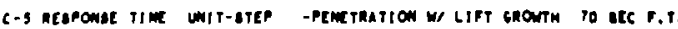

Fig. 18 - Indicial Response of Ist Bending Considering Penetration with Lift Growth - 70 sec. F.T. 


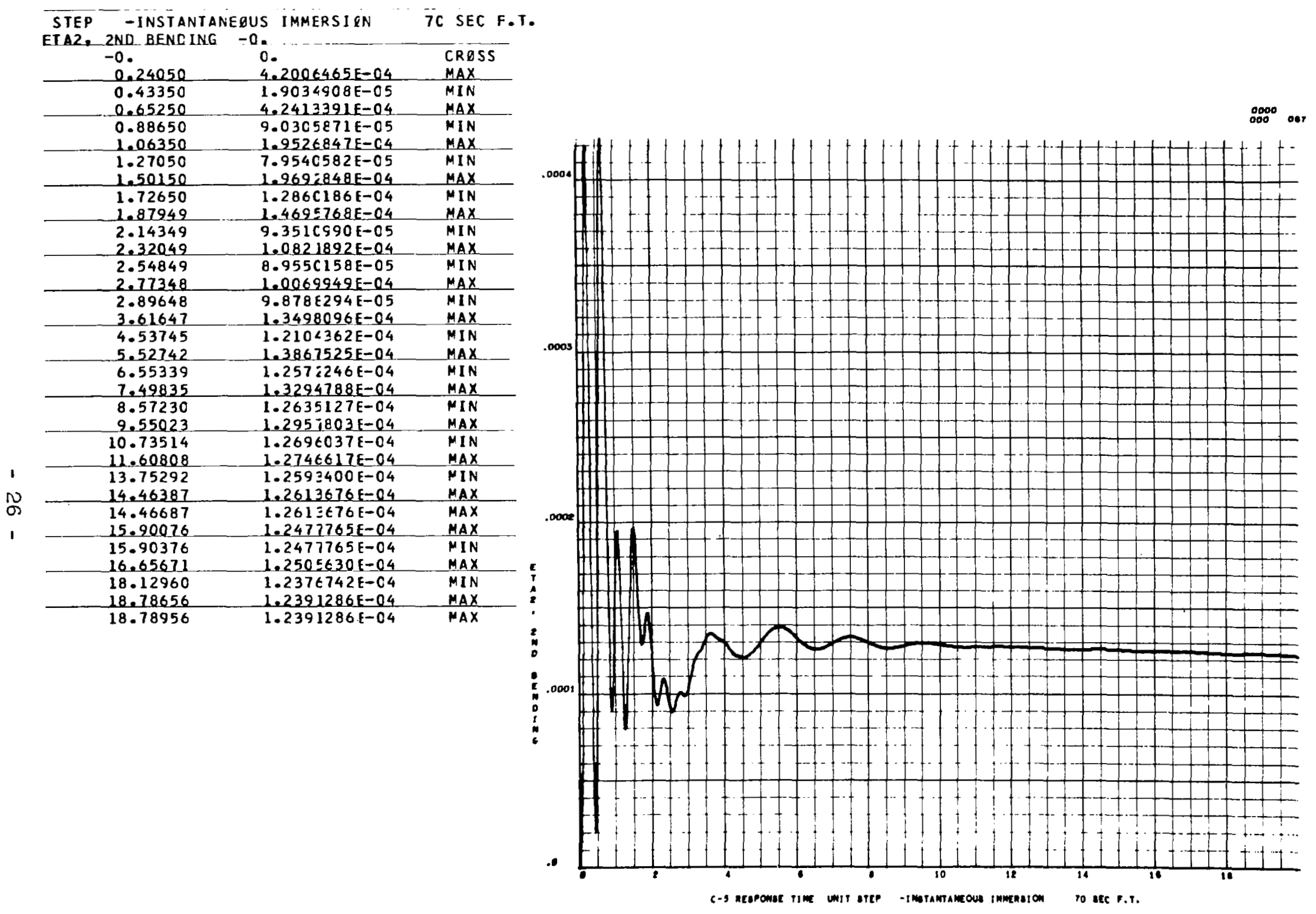

Fig. 19 - Indicial Response of 2nd Bending Considering Instantaneous Immersion - 70 sec. F.T. 


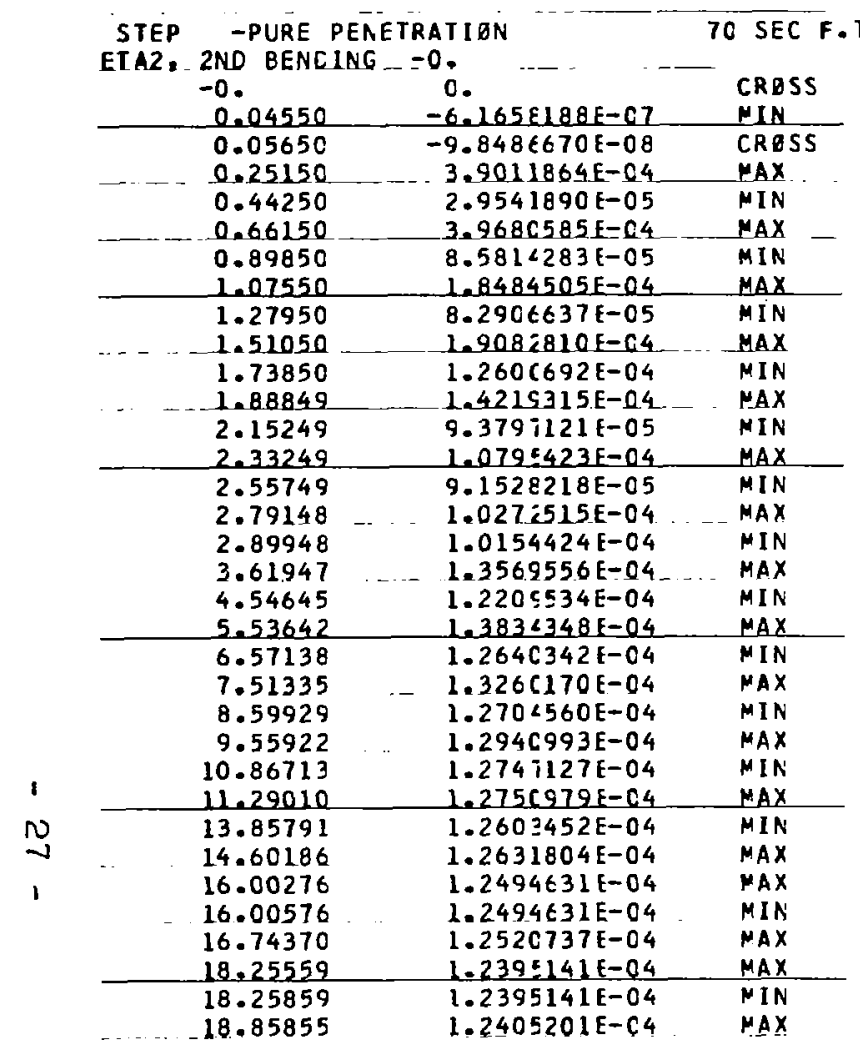

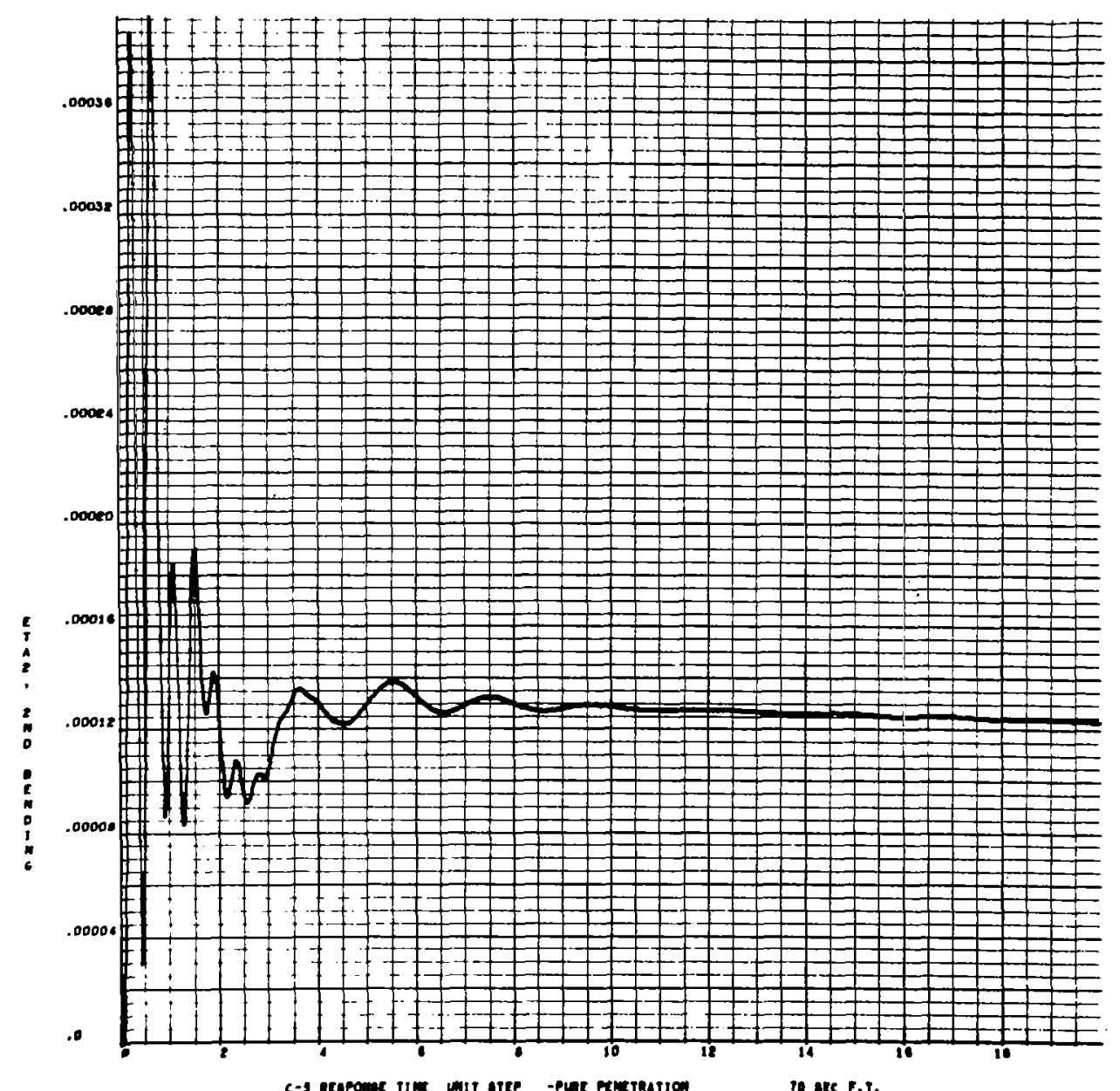

Fig. 20 - Indicial Response of 2nd Bending Considering Pure Penetration - 70 sec. F.T. 


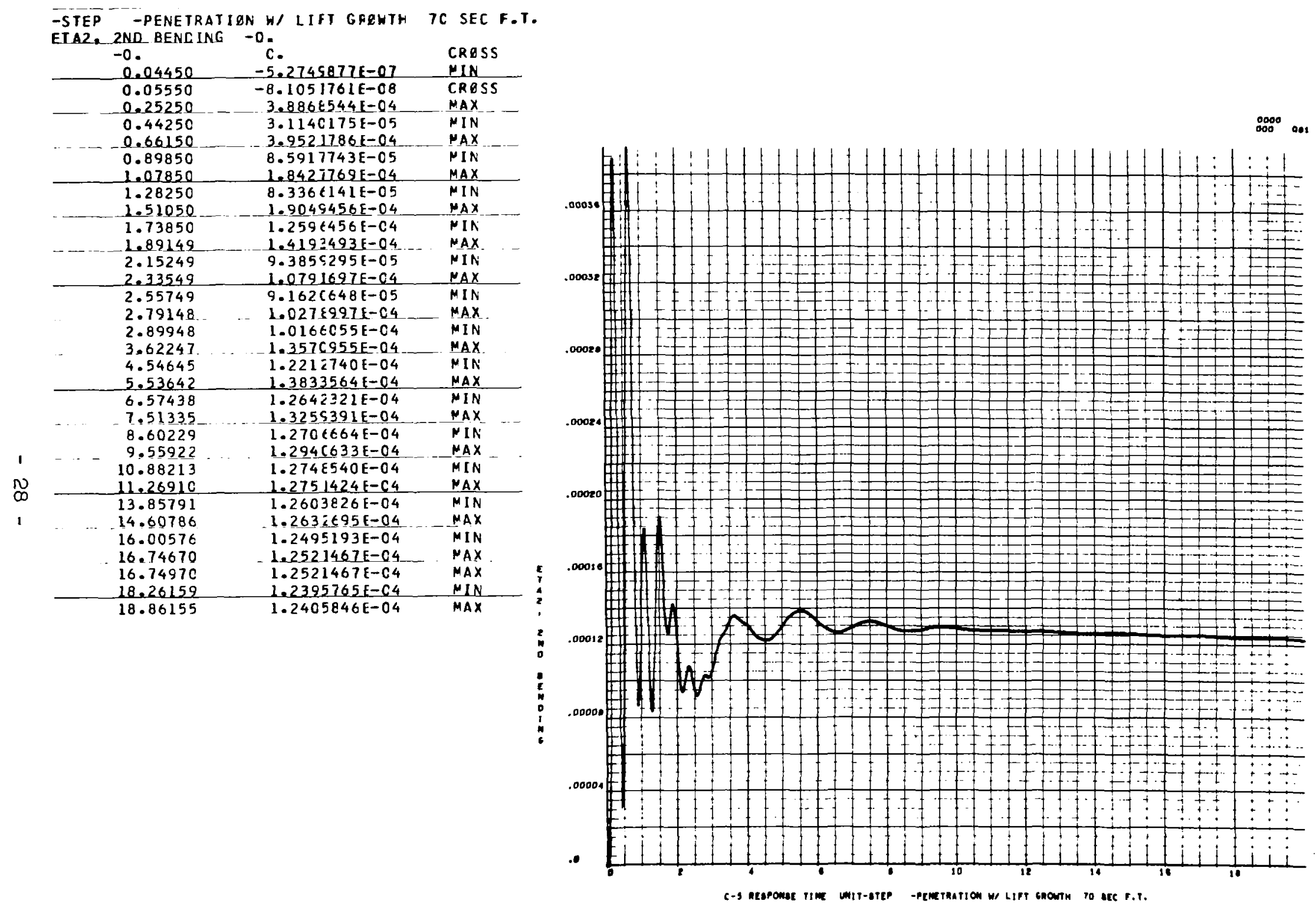

Fig. 21 - Indicial Response of 2nd Bending Considering Penetration with Lift Growth - 70 sec. F.T. 
STEP -INSTANTANEQUS IMMERSIRN

BEIAL. 1ST_CONTREL - 0 .

\begin{tabular}{|c|c|c|}
\hline $\begin{array}{r}-0.00050 \\
0.00050\end{array}$ & 0. & $\begin{array}{l}\text { CROSS } \\
\text { CROSS }\end{array}$ \\
\hline $\begin{array}{l}0.00050 \\
0.11250\end{array}$ & $0.4905891 E-C_{3}$ & $\begin{array}{l}\text { MIN } \\
\text { MAX }\end{array}$ \\
\hline $\begin{array}{l}0.22150 \\
0.23350\end{array}$ & $\begin{array}{r}3.0442425 \mathrm{E}-07 \\
-2.611 C 351 \mathrm{E}=05\end{array}$ & $\begin{array}{l}\text { CRESS } \\
\text { MUN }\end{array}$ \\
\hline $\begin{array}{l}0.24450 \\
0.41550\end{array}$ & $\begin{array}{r}-2.5691210 \mathrm{E}-06 \\
1.6102168 \mathrm{E}=03\end{array}$ & $\begin{array}{l}\text { CRQSS } \\
\text { MAX }\end{array}$ \\
\hline $\begin{array}{l}0.61350 \\
0.79350\end{array}$ & $\begin{array}{l}8.3336432 E-04 \\
1.2767245 E-03\end{array}$ & $\begin{array}{l}\text { MIN } \\
\text { MAXX }\end{array}$ \\
\hline $\begin{array}{l}1.05450 \\
1.25850\end{array}$ & $\begin{array}{l}3.2466637 \mathrm{E}-04 \\
6.5305269 \mathrm{E}-\mathrm{C} 4\end{array}$ & $\begin{array}{l}\text { MIN } \\
\text { MAX }\end{array}$ \\
\hline $\begin{array}{l}1.43250 \\
1.64250\end{array}$ & $\begin{array}{l}5.1621032 \mathrm{E}-\mathrm{O} 4 \\
7.015 \mathrm{E} 93 \mathrm{E}-04\end{array}$ & MIN \\
\hline $\begin{array}{r}1.93949 \\
2.02349 \\
2.30849 \\
2.49149 \\
2.68649 \\
3.72447\end{array}$ & $\begin{array}{l}4.240 \div 112 \mathrm{E}-04 \\
4.3095941 \mathrm{E}-04 \\
3.092<222 \mathrm{E}-04 \\
3.400 \mathrm{C} 468 \mathrm{E}-04 \\
3.1128705 \mathrm{E}-04 \\
5.3220 \mathrm{C}\end{array}$ & $\begin{array}{l}\text { MIN } \\
\text { MAX } \\
\text { MIN } \\
\text { MAX } \\
\text { MAX } \\
\text { MAX }\end{array}$ \\
\hline $\begin{array}{l}4.44145 \\
5.48542 \\
6.50239 \\
7.39036 \\
8.42531 \\
9.34624\end{array}$ & $\begin{array}{l}4.8288 C 97 \mathrm{E}-04 \\
5.593 \leq 162 \mathrm{E}-04 \\
5.0732055 \mathrm{E}-04 \\
5.3263019 \mathrm{E}-04 \\
5.0689631 \mathrm{E}-04 \\
5.203 C 047 \mathrm{E}-04\end{array}$ & $\begin{array}{l}\text { MIN } \\
\text { MAX } \\
\text { MIN } \\
\text { MAX } \\
\text { MIN } \\
\text { MAX }\end{array}$ \\
\hline $\begin{array}{l}10.38416 \\
11.24810 \\
12.39702 \\
13.06592\end{array}$ & $\begin{array}{l}5.083<611 \mathrm{E}-04 \\
5.1347905 \mathrm{E}-04 \\
5.070 \mathrm{C} 38 \mathrm{E}-04 \\
5.081 \mathrm{C} 810 \mathrm{E}-04\end{array}$ & $\begin{array}{l}\text { MIN } \\
\text { MAX } \\
\text { MIN } \\
\text { MAX }\end{array}$ \\
\hline
\end{tabular}

TC SEC F.T.

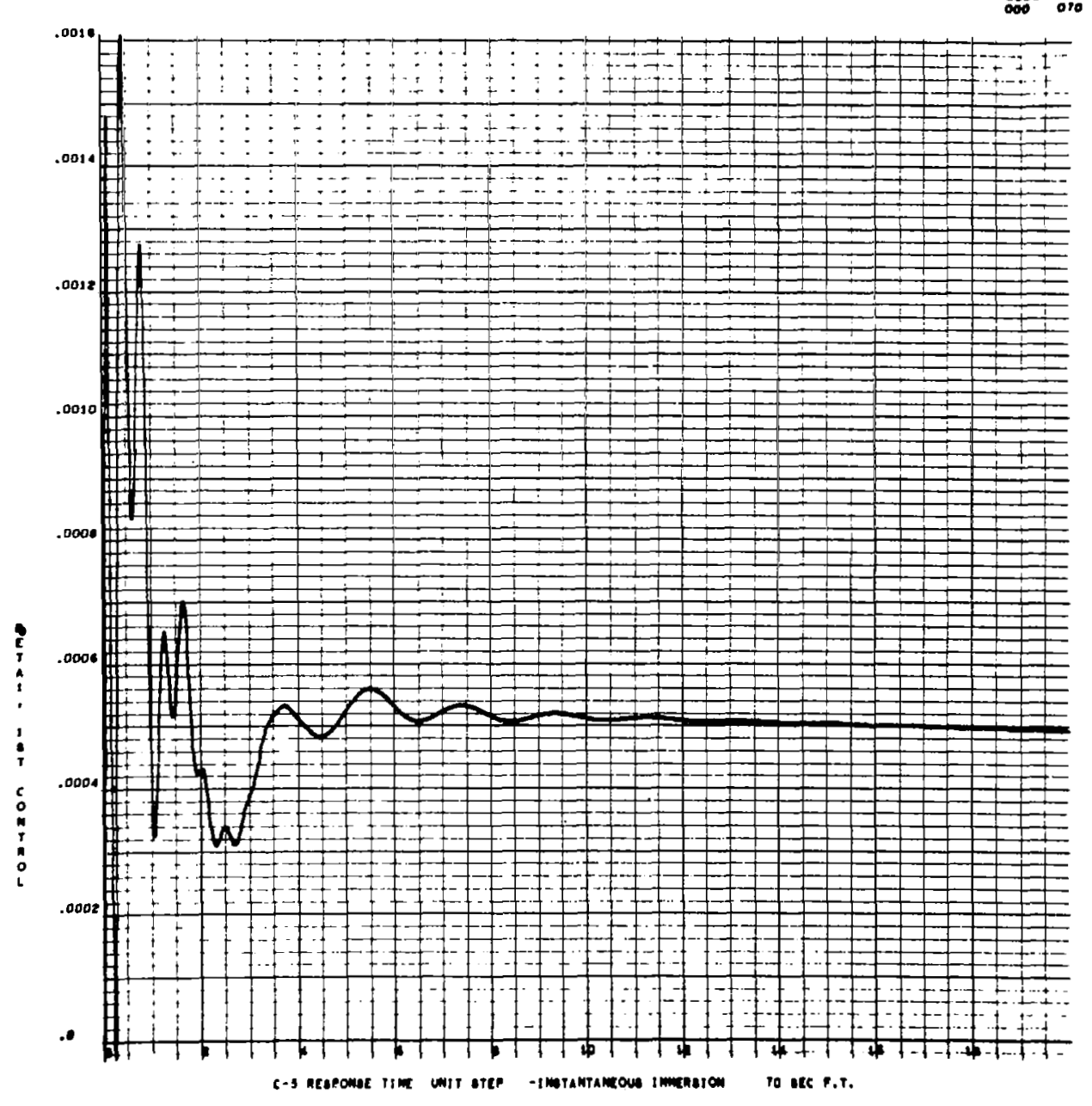

Fig. 22 - Indicial Response of lst Control Considering Instantaneous Immersion - 70 sec. F.T. 


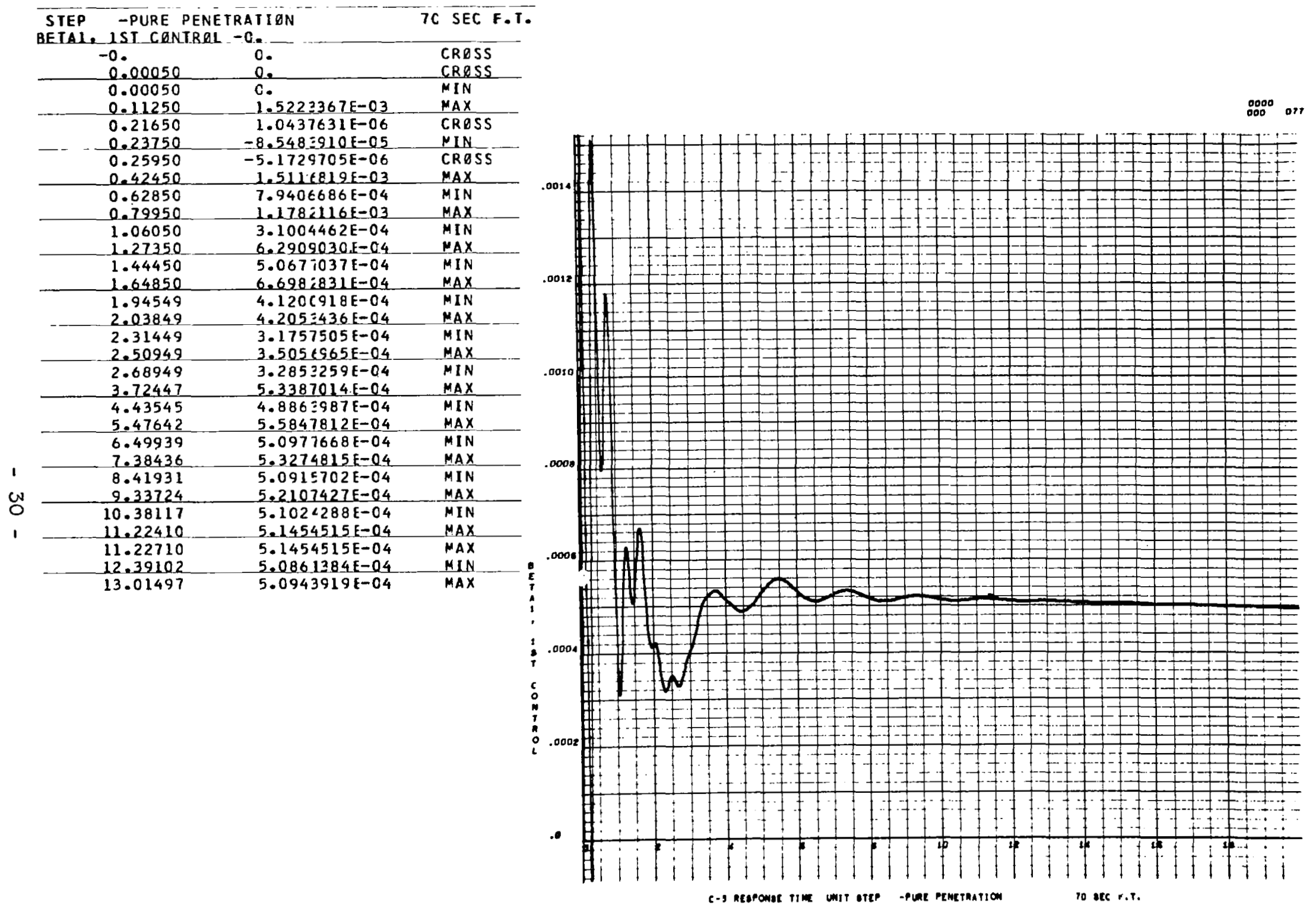

Fig. 23 - Indicial Response of Ist Control Considering Pure Penetration - 70 sec. F.T. 


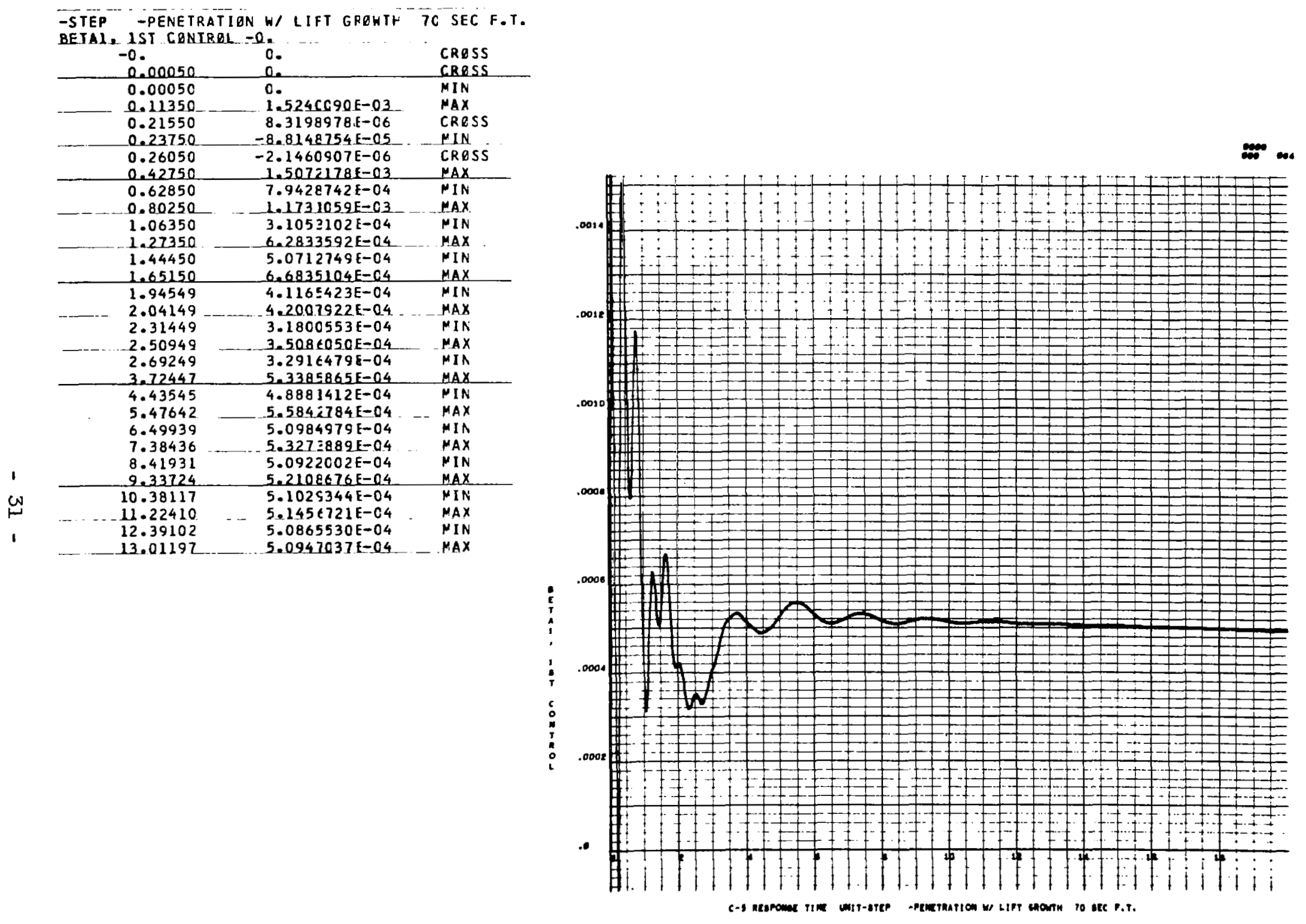

Fig. 24 - Indicial Response of Ist Control Considering Penetration with Lift Growth - 70 sec. F.T. 
The discussion of the effects and importance of penetration and growth of lift on the indicial and inpulsive responses of the vehicle will be presented in a later section.

There is, however, some information readily derivable from the indicial and impulsive responses which has a direct bearing on the calculation of the wind-induced responses. The evaluation of the wind-induced responses can be determined from either the indicial or impulsive responses. An important feature in this choice is the convergence characteristic of the response. From Figs. 1 through 18, it is seen that the indicial responses do not converge satisfactorily within the 20-sec. record. The path or drift root component has a predominant influence on the slow convergence of the indicial responses. The impulsive responses, hovever, are not as greatly influenced by this component and do converge satisfactorily within the record. Thus, the impulsive responses were selected for use in the calculation of the wind-induced responses. The evaluation of the wind-induced responses is discussed in the next section.

\section{Wind-Induced Responses}

This scction presents the method used and procedures followed in computing the wind-induced responses of the Saturn C-5 configuration. A sample of the numerical calculations is presented for 1llustrative purposes.

The responses of the vehicle to any side wind input are formulated as Duhamel integrals $\{$ see [4] $\}$. The kernel function of these integrals can be written in terms of either the indicial or impulsive responses of the system. From the discussion given in the previous section, it is advantageous to compute the wind-induced responses in terms of the impulsive responses.

Thus, from [4], the wind-induced responses are given by

$$
R(t)=\int_{0}^{t} R_{i}(t-\tau) v_{y}(\tau) d \tau
$$

where $R_{i}(\tau)$ is the ith coordinate response to a unit impulse wind, $v_{y}(\tau)$ is an arbitrary side wind profile and $R(t)$ is the ith coordinate response to the wind, $v_{y}(t)$. 
The wind velocity used in the above integral was available as a function of altitude. The profile was interpreted in terms of time by using a constant missile velocity associated with the midpoint of the altitude band. This, in essence, produced a slight shift in the effective wind frequencies at the extremes of the altitude interval.

The responses of the Saturn $C-5$ to two wind profiles* were computed. The wind profiles used were: the West - East components of 8116 and 4768 .** (In this report 8116 is referred to as profile 1 ; and 4768 is referred to as profile 2.) These profiles had been measured by a modified spherical balloon (Jimsphere) - radar technique and were tabulated at 25-meter increments. The 8116 profile represents a moderately severe wind environment (maximum wind velocity of 46.3 meters/sec at an altitude of 13,200 meters). The 4768 profile represents a mild wind environment (naximum wind velocity of 17.8 meters/sec at an altitude of 19,000 meters).

The vehicle responses to the two profiles were computed at numerous altitude points during the flight trajectory. The responses of the following generalized coordinates were computed:

$$
\begin{aligned}
& \text { Translation, } y_{0} \\
& \text { Rotation, } \phi \\
& \text { First bending, } \eta_{1} \\
& \text { Second bending, } \eta_{2} \\
& \text { First sloshing, } \xi_{1} \\
& \text { Second sloshing, } \xi_{2} \\
& \text { Control deflection, } \beta_{C}
\end{aligned}
$$

* The wind data were supplied by George $C$. Marshall space Flight Center. * Wind values for profile 8116 were given between altitudes of 2,350 and 19,525 meters; values for profile 4768 were given between 450 and 19,700 meters. 
The wind-induced responses were evaluated in five flight time bands. Each band was initially chosen to encompass $10 \mathrm{sec}$. of flight time with missile parameters evaluated at the band midpoint. Subsequently, each of the actual calculations was extended to a length of $15 \mathrm{sec}$. so that $5 \mathrm{sec}$. of overlap were available for comparison. The comparisons were a practical test of the use of frozen coefficients. The agreement of response values in the overlap regions was, in general, satisfactory. As expected, the high-frequency responses showed better agreement than low-frequency responses.

The correspondence between the altitudes and flight time bands used in the numerical calculation of the wind-induced responses is presented in Table II. The increment used in each band to calculate the responses is also given. These increments were chosen to provide sufficient calculated points to adequately define the highest frequency component.

\section{TABIE II}

AITITUDE BANDS USED IN CAICUIATING WIND-INDUCED RESPONSES

Bands

\begin{tabular}{cccc}
\hline $\begin{array}{c}\text { Flight Time } \\
\text { (sec.) }\end{array}$ & $\begin{array}{c}\text { Flight Time } \\
\text { Interval } \\
\text { (sec.) }\end{array}$ & $\begin{array}{c}\text { Altitude Interval } \\
\text { (meters) }\end{array}$ & $\begin{array}{c}\text { Response Increment } \\
\text { (meters) }\end{array}$ \\
\hline 50 & 45 to 60 & 3,594 to 7,104 & 8.33 \\
60 & 55 to 70 & 5,787 to 10,200 & 12.5 \\
70 & 65 to 80 & 8,573 to 13,940 & 12.5 \\
80 & 75 to 90 & 11,987 to 18,361 & 12.5 \\
90 & 85 to 92.5 & 16,063 to 19,525 & 12.5
\end{tabular}

a/ It was not possible to obtain an adequate description of the slowly converging responses at the lowest end of some of the intervals because the wind profile did not extend to a sufficiently low altitude. 
To avold burdening the report with an excessive amount of data, only a representative quantity of the wind-induced response data is presented at this time. Plots of the first bending, $\eta_{1}$, second bending, $\eta_{2}$, and control deflection, $f_{C}$ (noted by first control) responses to profile 8116 (noted by profile 1) are given in Figs. 25 through 33 for the altitude band, 8,573 to 13,940 meters. The 70-sec. impulsive responses were used in computing these responses. For each coordinate, the first figure reflects the effect of instantaneous immersion; the second figure reflects the effect of pure penetration;* and the third reflects the effect of penetration with lift growth.

The numerics presented alongside the plots in each figure pertain to an analysis of the response curves. The format of the information presented is as follows:

$\begin{array}{lcc}\text { Response } & \text { Response } & \text { Response } \\ \text { Altitude } & \text { Value } & \text { Maximum, Minimum or } \\ & & \text { Crossing }\end{array}$

The maximum and minimum values, indicated by a check mark, define the approximate envelope of the extreme excursians of the response. These values were used in the analysis of the wind-induced responses discussed in the next section.

IV. METHOD USED IN ANALYSIS OF EFFECTS AND IMFORTANCE OF FENETRATION AND GROWTH OF LIFT ON MISSIIE RESPONSE

The analysis of the indicial and impulsive responses is largely qualitative.

The three aerodynamic representations yield sinilar responses to the indicial wind. The responses to the unit impulse wind are also very much alike. Samples of these responses are shown in the section, "Method of Solution for the Indicial and Irupulsive Responses." The most noticeable differences occur during the highly oscillatory response shortly after immersion in the gust.

* An undesired translation of altitude was made in the calculation of the responses to profile 1 considering pure penetration effects. The altitudes recorded in the plots and tabulations should be reduced by 100 meters. 
PREFILE 1. ALIITUDE VS. RESPGNSE-INSTANTANE DUS IMMERSION 70 SEC F.T. ETAL, IST BENUING

\begin{tabular}{|c|c|c|c|}
\hline $\begin{array}{l}\text { ALTI TUDE } \\
8650 .\end{array}$ & $\begin{array}{l}\text { RESPQNSE } \\
1.4404573 E-02\end{array}$ & TYPE & SEOO \\
\hline 9013. & $\begin{array}{l}1.6436338 E-02 \\
1.5637597 \mathrm{E}-02\end{array}$ & $\begin{array}{l}\text { MAX } \\
\text { MIN }\end{array}$ & \\
\hline 9338 & $1.7740874 \mathrm{E}-02$ & $\operatorname{MAX}$ & \\
\hline 9550. & $1.4863462 E-02$ & MIN & \\
\hline 9800 . & $1.7691638 \mathrm{E}-02$ & MAX & \\
\hline 10100. & $1.4604546 \mathrm{E}-02$ & MIN & r \\
\hline 10300 & $\begin{array}{l}1.7447110 \mathrm{E}-02 \\
1.6632714 \mathrm{E}-02\end{array}$ & $\operatorname{MAX}$ & \\
\hline 10588. & $1.8907347 \mathrm{E}-02$ & $-\frac{\min }{\operatorname{MAX}}$ & \\
\hline 10800. & $1.4053984 E-02$ & MIN & \\
\hline 10975 . & $1.6250993 \mathrm{E}-02$ & MAX & \\
\hline 11213. & $1.3777776 \mathrm{E}-02$ & MIN & \\
\hline 11375 & $1.4129932 \mathrm{E}-02$ & $\operatorname{MAX}$ & \\
\hline 11488. & $1.3703871 \mathrm{E}-02$ & MIN & \\
\hline 11725 . & $1.5146002 E-02$ & $\max$ & \\
\hline 11763. & $1.5128871 E-02$ & MIN & \\
\hline 12138. & $2.1379113 \mathrm{E}-02$ & $\operatorname{MAX}$ & \\
\hline 12363. & $1.9267983 E-02$ & MIN & L \\
\hline 12625 . & $2.2913437 E-02$ & $\operatorname{MAX}$ & . \\
\hline 12925. & $1.9371118 \mathrm{E}-02$ & MIN & \\
\hline 13200 . & $2.0593875 E-02$ & $\operatorname{MA}^{-} x^{-1}$ & \\
\hline 1357 & $1.9190341 \mathrm{E}=02$ & $\operatorname{MIN}$ & \\
\hline 13 & 2.0111562 & $\max$ & \\
\hline
\end{tabular}

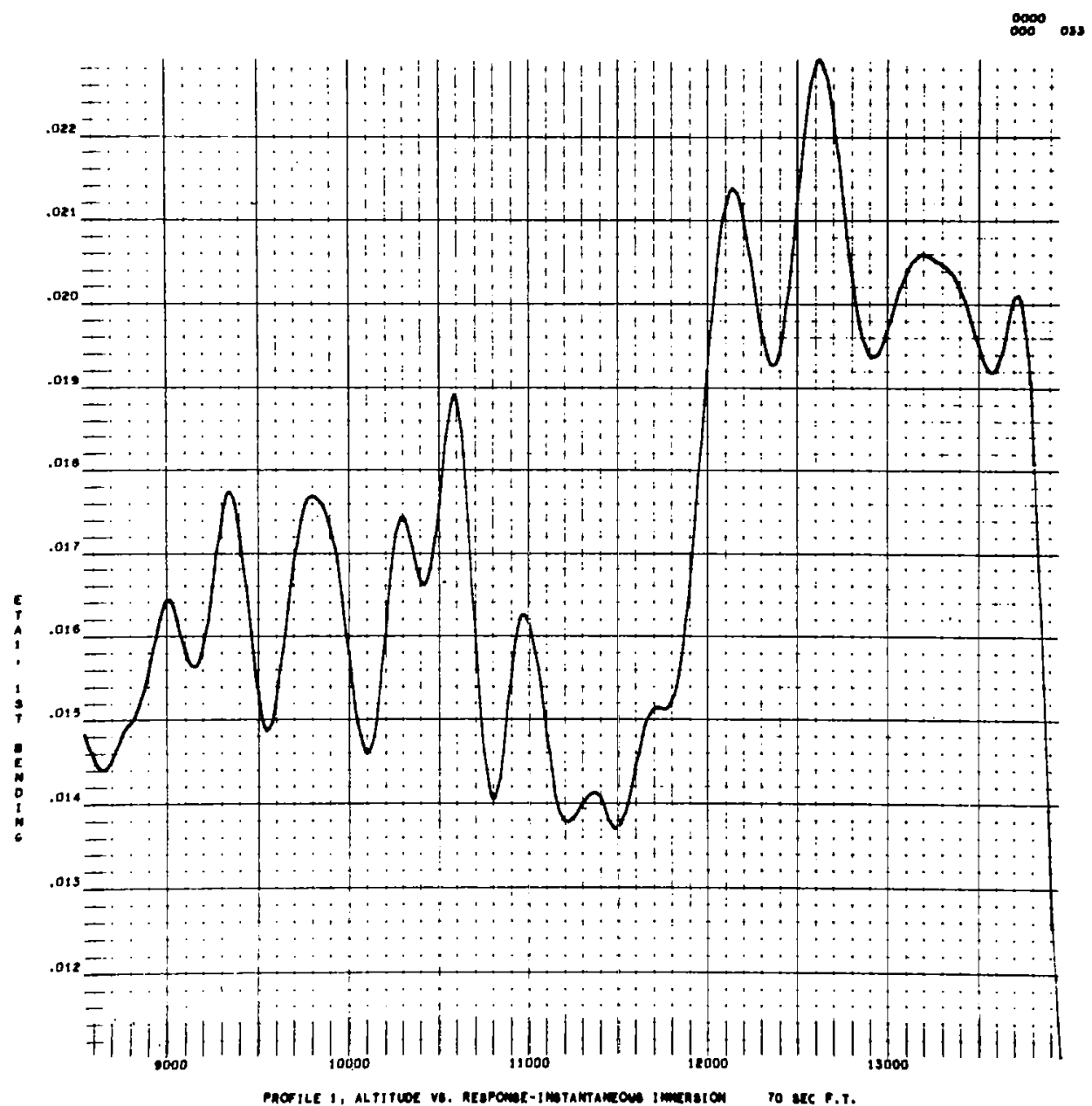

Fig. 25 - Wind-Induced Response of lst Bending Considering Instantaneous

Immersion - $70 \mathrm{sec}$. F.T. Band 
PRgFILE 1, ALIITUDE VS. RESPgNSE-PURE PENETRATIGN ETAL, IST BENDING

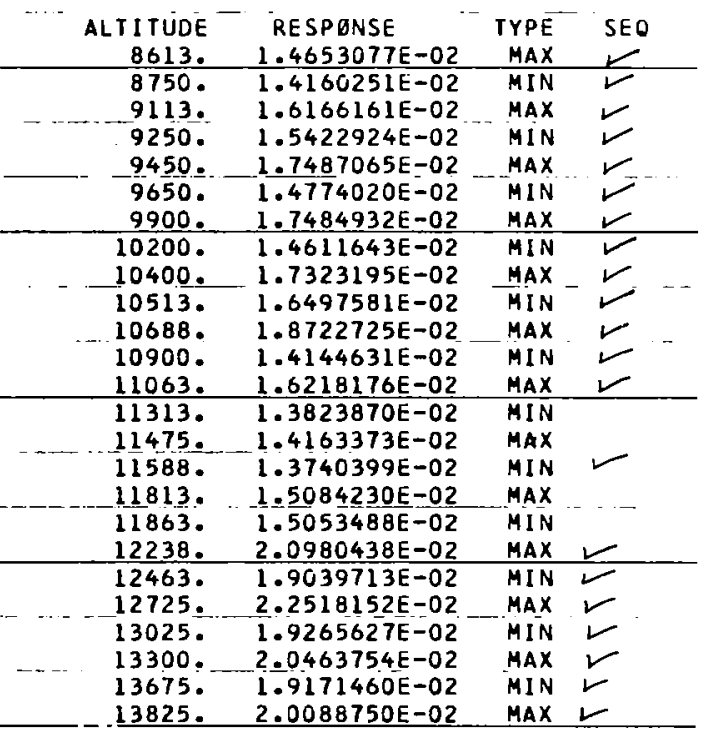

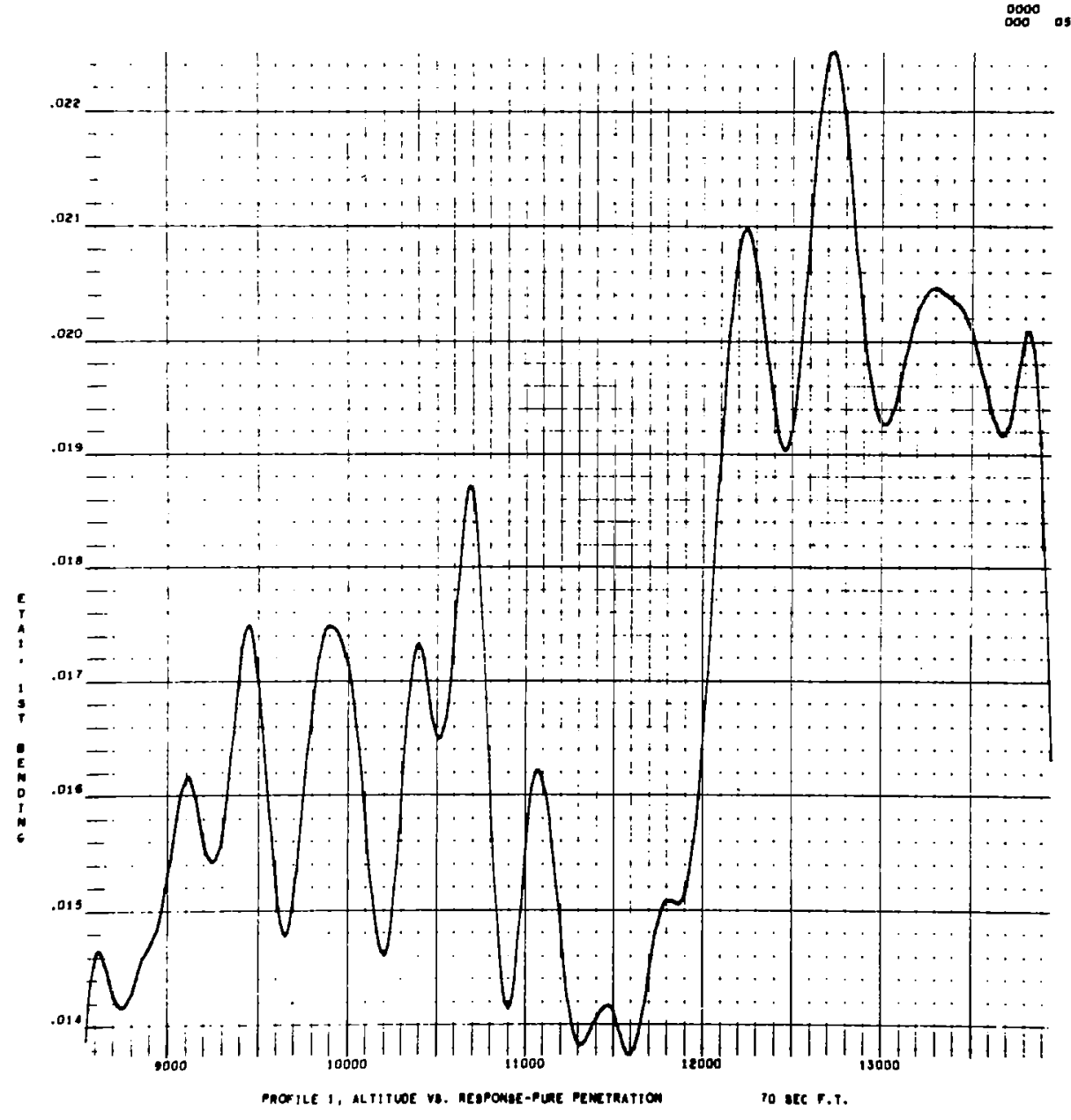

Fig. 26 - Wind-Induced Response of lst Bending Considering Pure

Penetration - $70 \mathrm{sec}$. F.T. Band 
PROFILE 1, ALTITUDE VS. RESPQNSE-PENETRATIEN WI LIFT GRQWTH 70 SEC F.T.

ETAL, LST BENDING

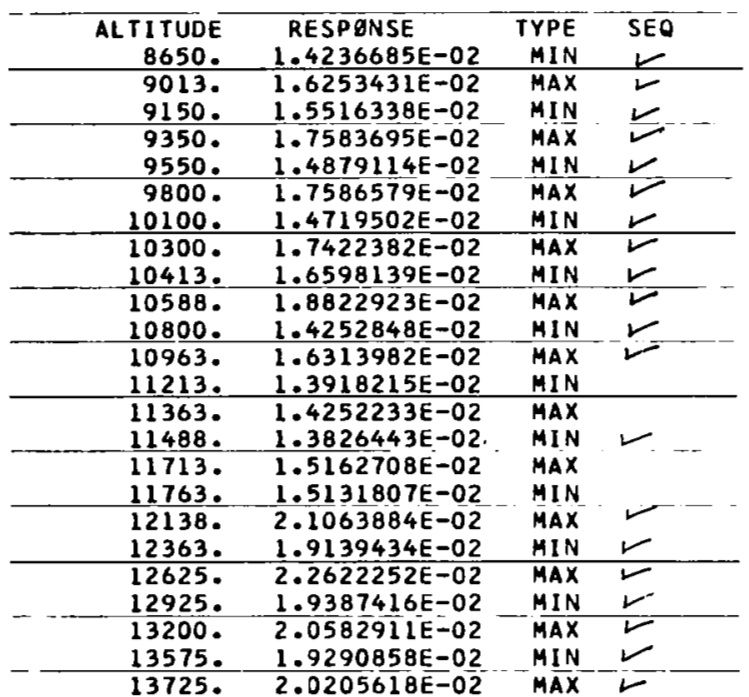

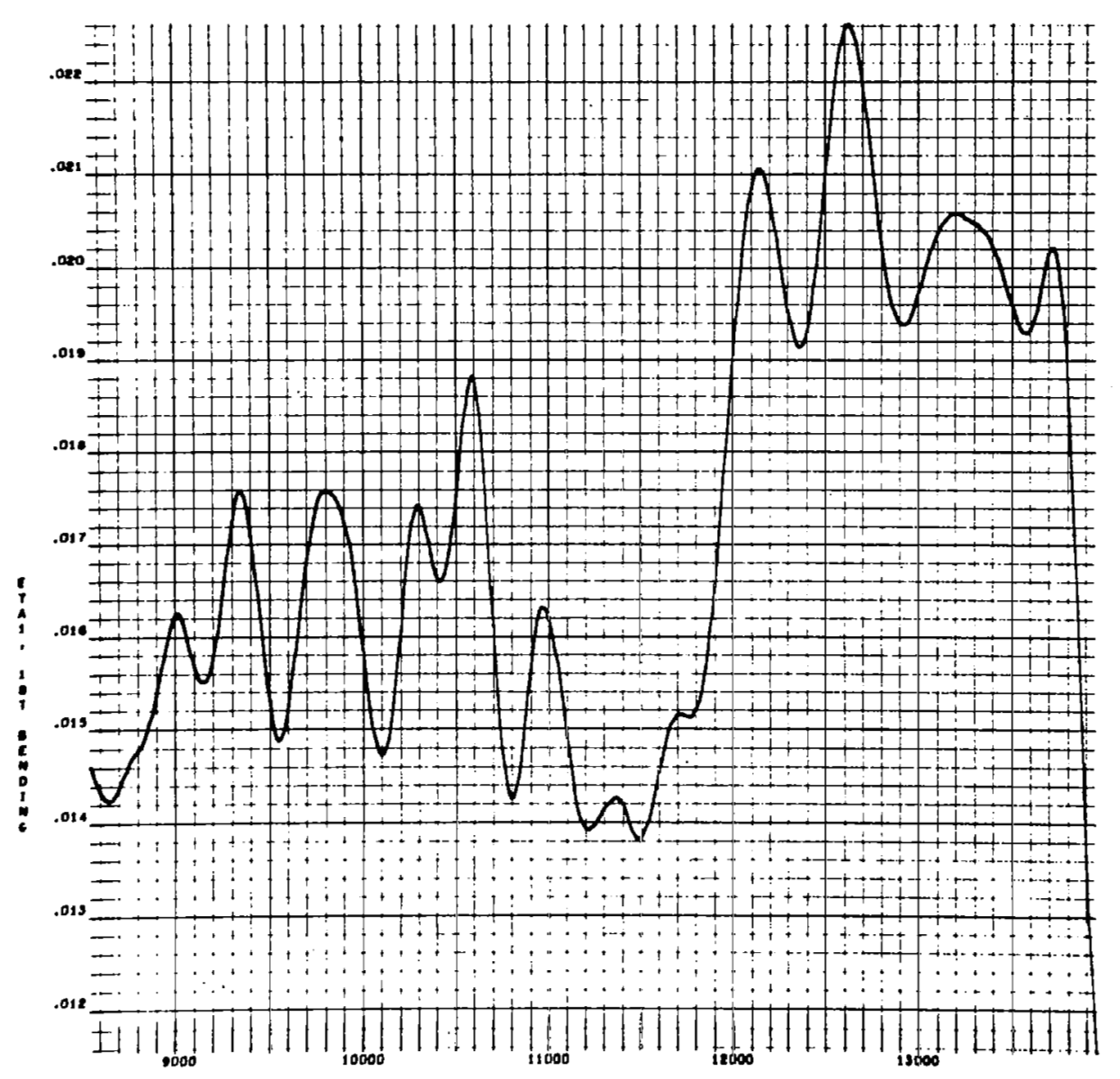

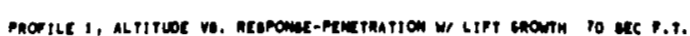

Fig. 27 - Wind-Induced Response of lst Bending Considering Penetration

With Lift Growth - $70 \mathrm{sec}$. F.T. Band 


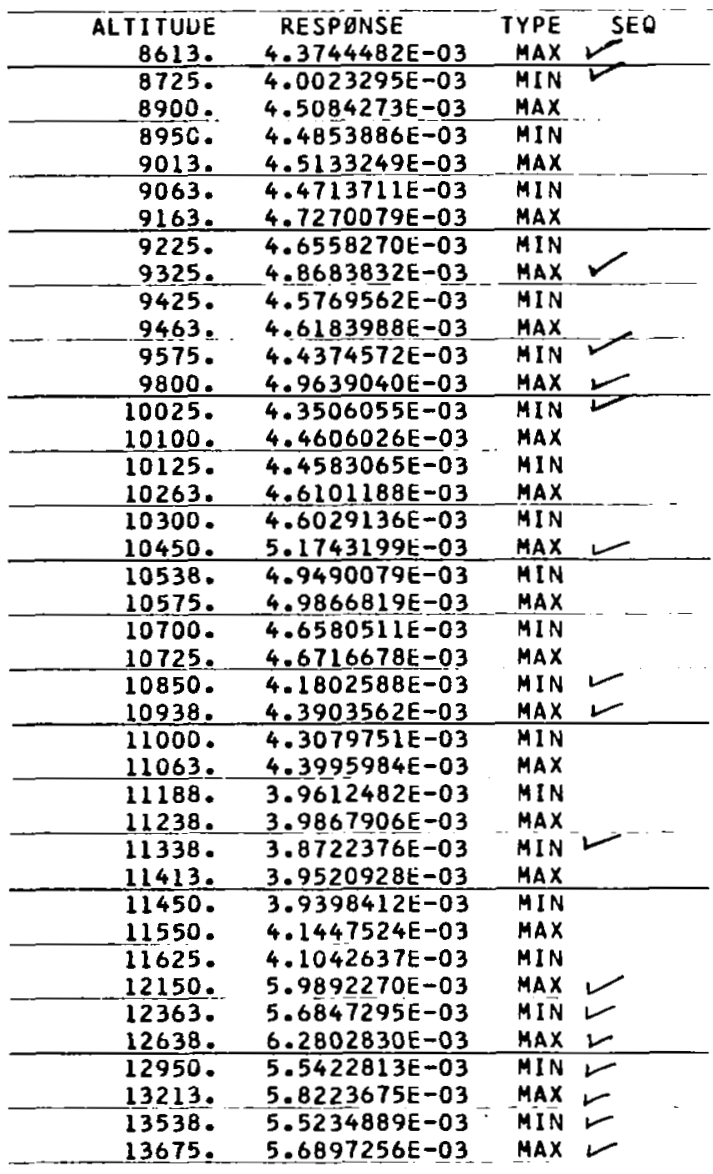

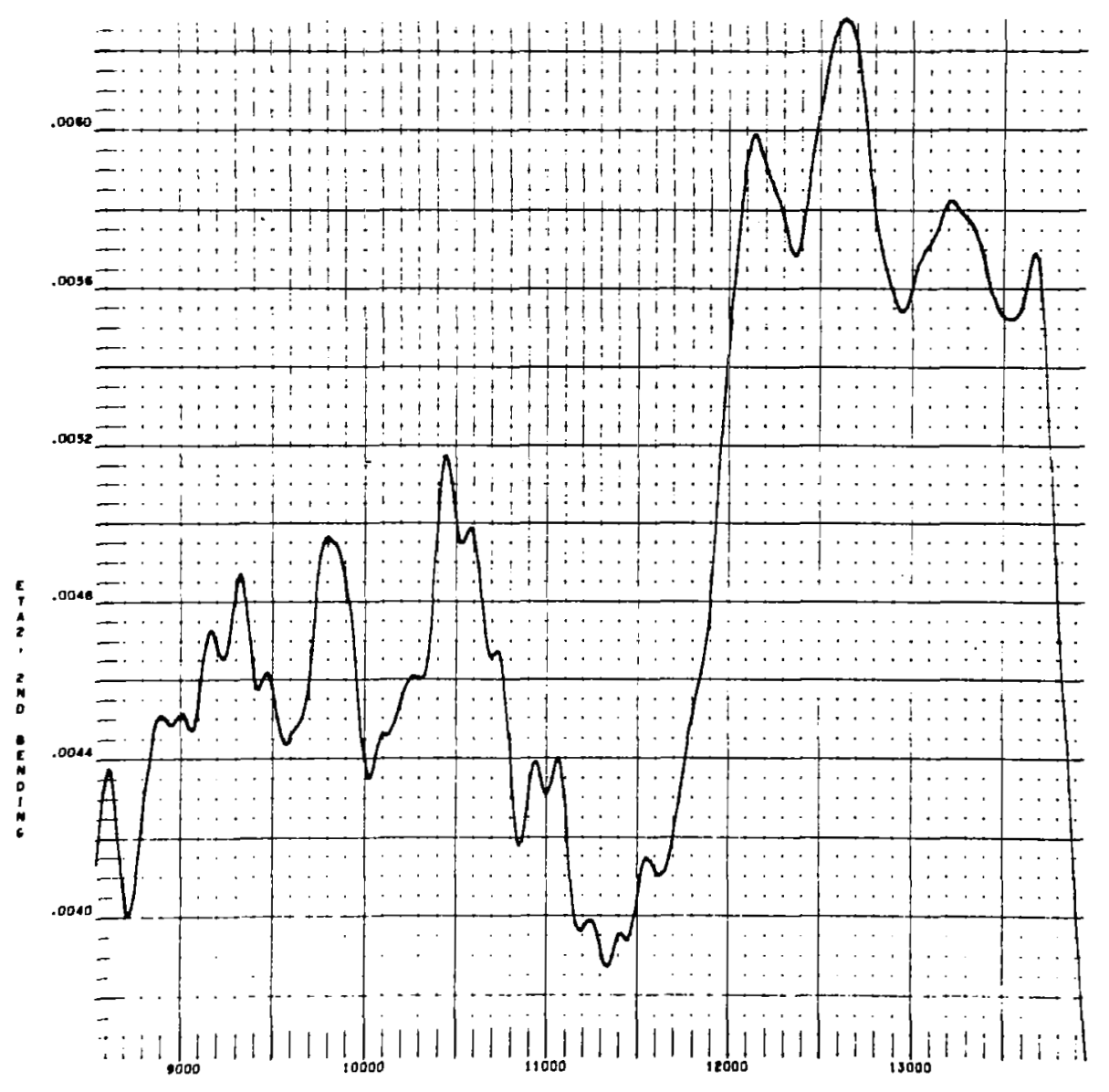

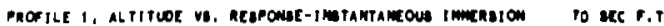

Fig. 28 - Wind-Induced Response of 2nd Bending Considering Instantaneous Immersion - $70 \mathrm{sec}$. F.T. Band 
PRBFILE 1, ALTITUUE VS. RESPBNSE-PURE PENETRATIGN 70 SEC F.T.

ETA2. 2ND BENDING

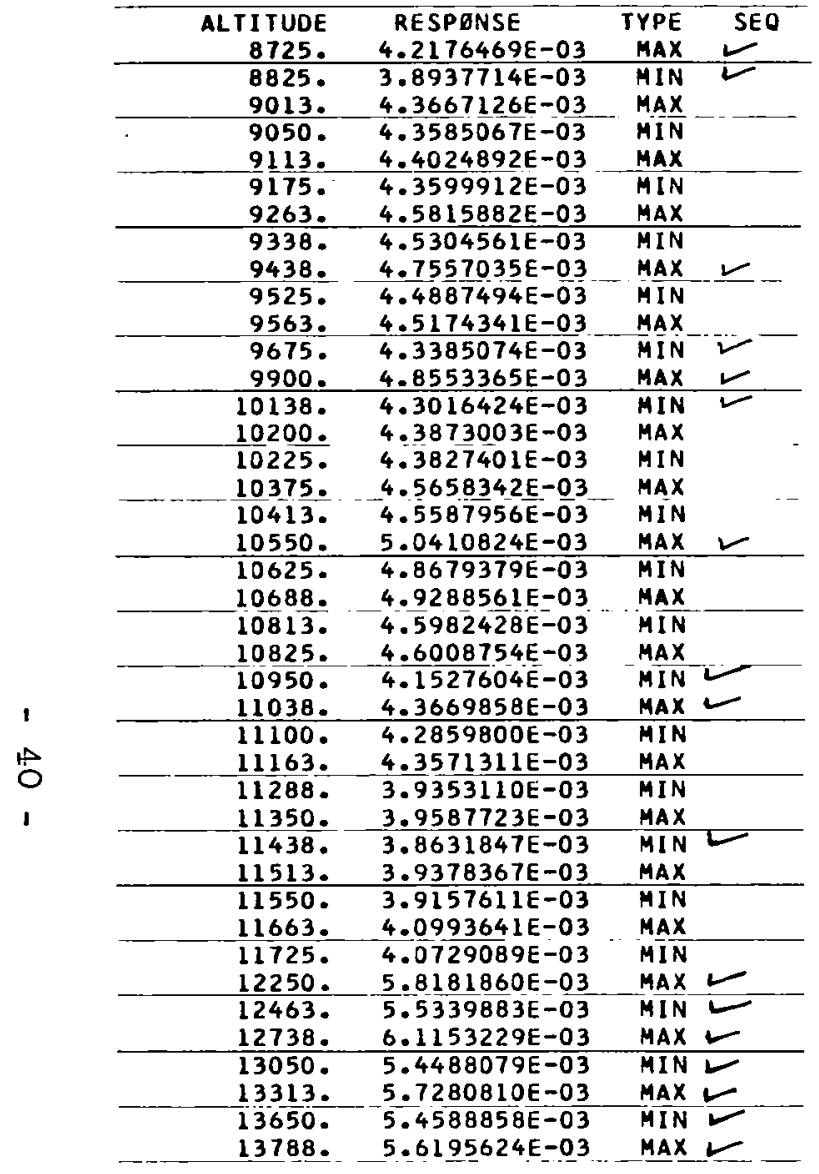

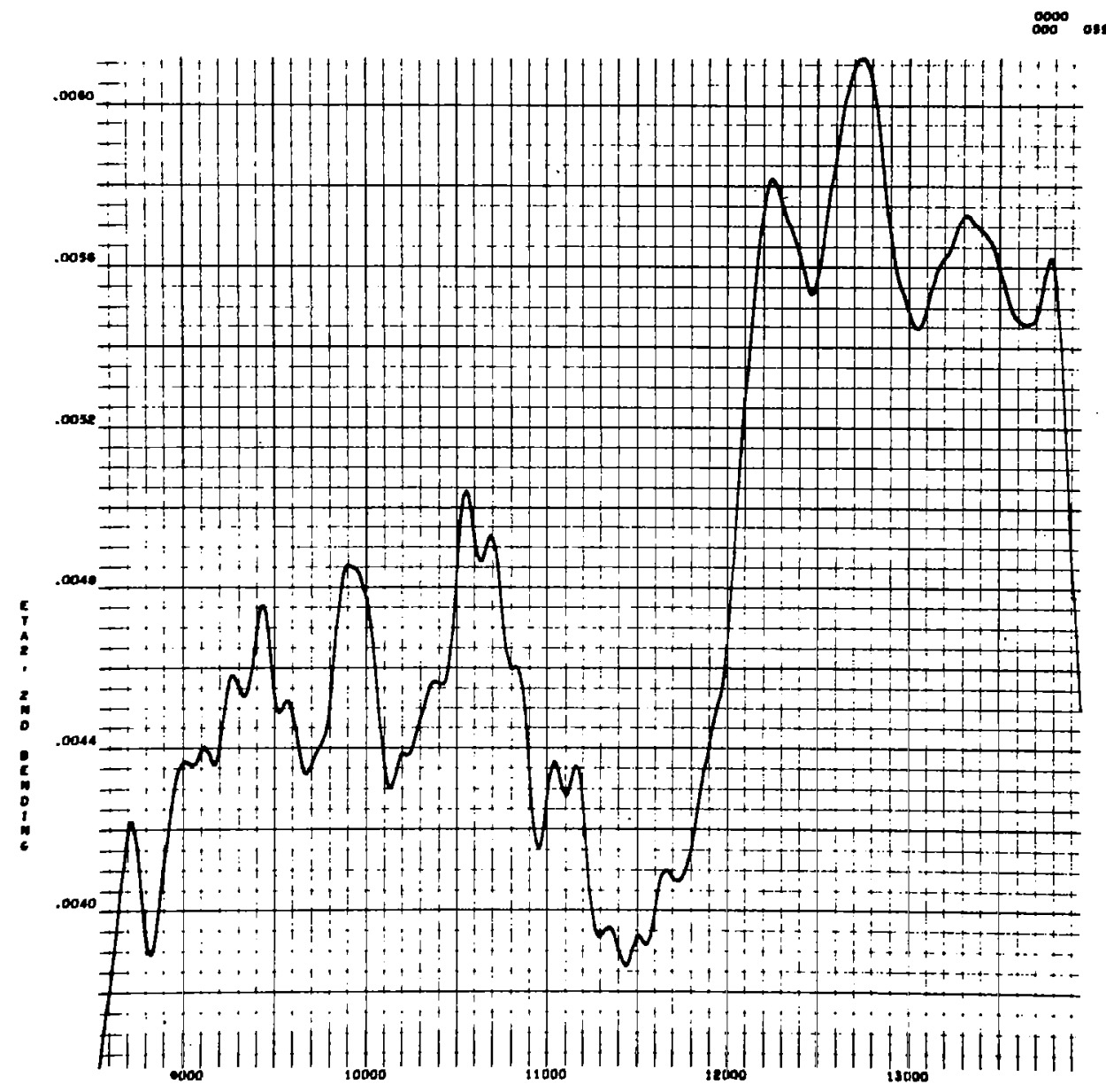

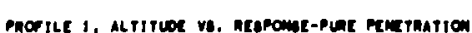

To acc r.t.

Fig. 29 - Wind-Induced Response of 2nd Bending Considering Pure

Penetration - $70 \mathrm{sec}$. F.T. Band 
PRQFILE 1, ALTITUDE VS. RESPGNSE-PENEIRATION W/ LIFT GREWTH TO SEC F.T.

ETA2, 2ND BENDING

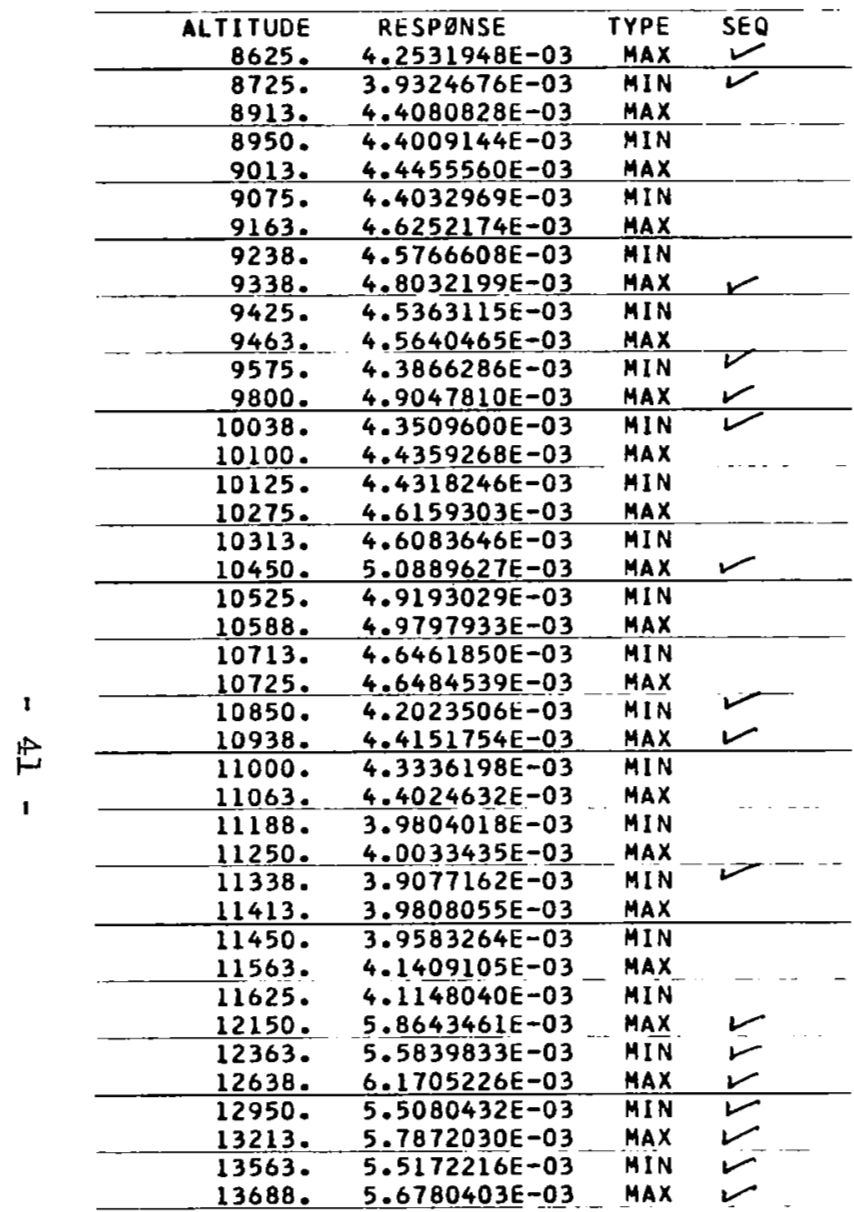

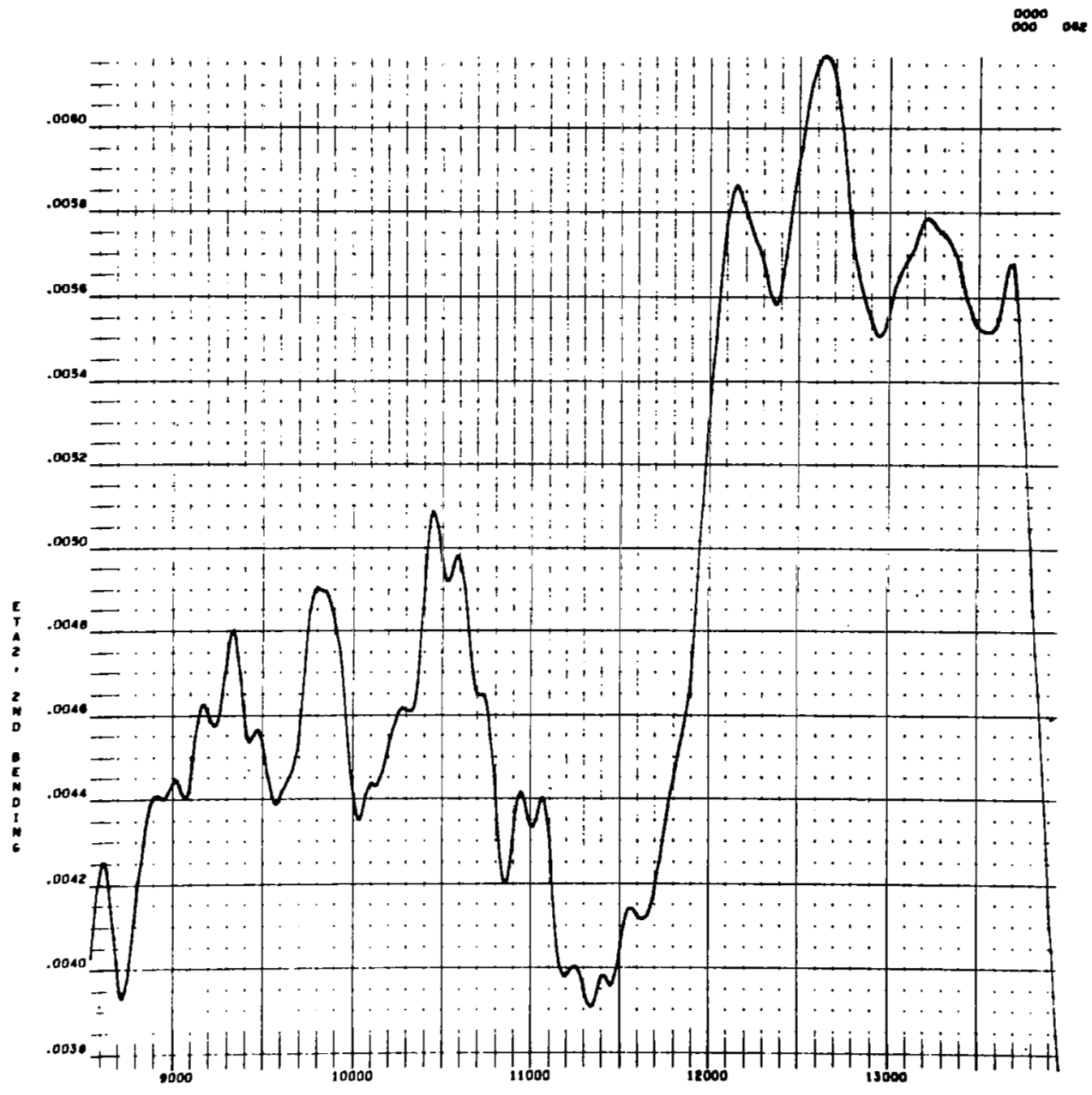

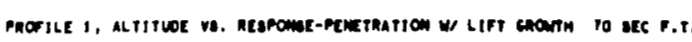

Fig. 30 - Wind-Induced Response of 2nd Bending Considering Penetration

With Lift Growth - $70 \mathrm{sec}$. F.T. Band 
PRQFILE 1. ALTITUDE VS. RESPQWNSE-INSTANIANEQUS IMMERSION BETAL, IST CONTREL

70 SEC F.T.

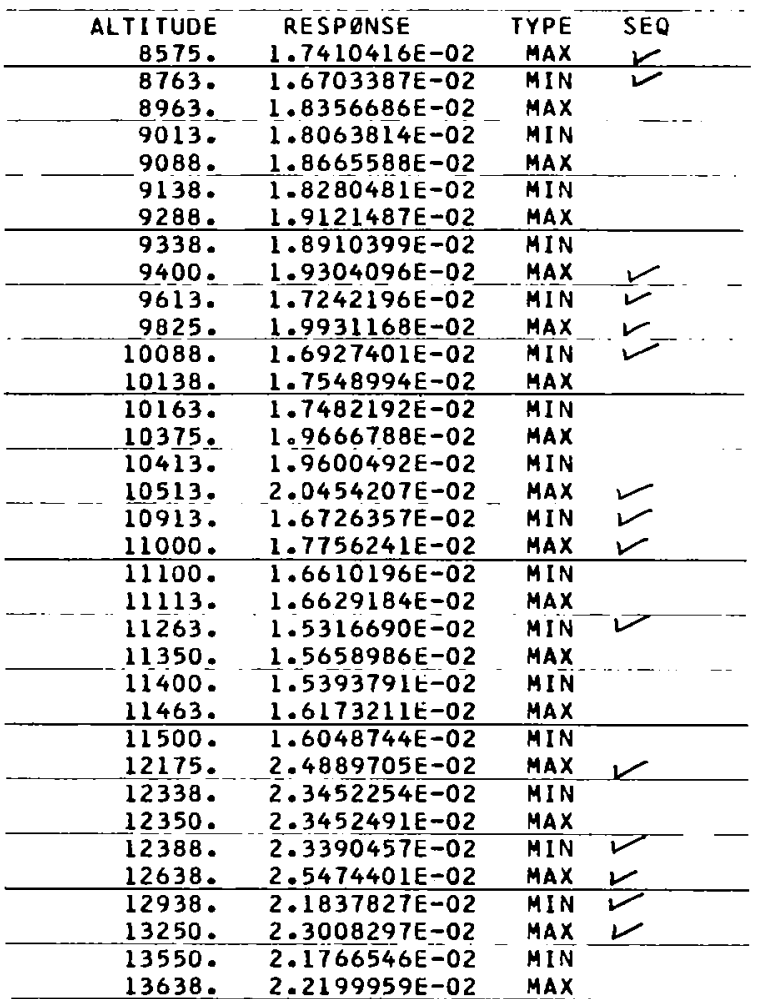

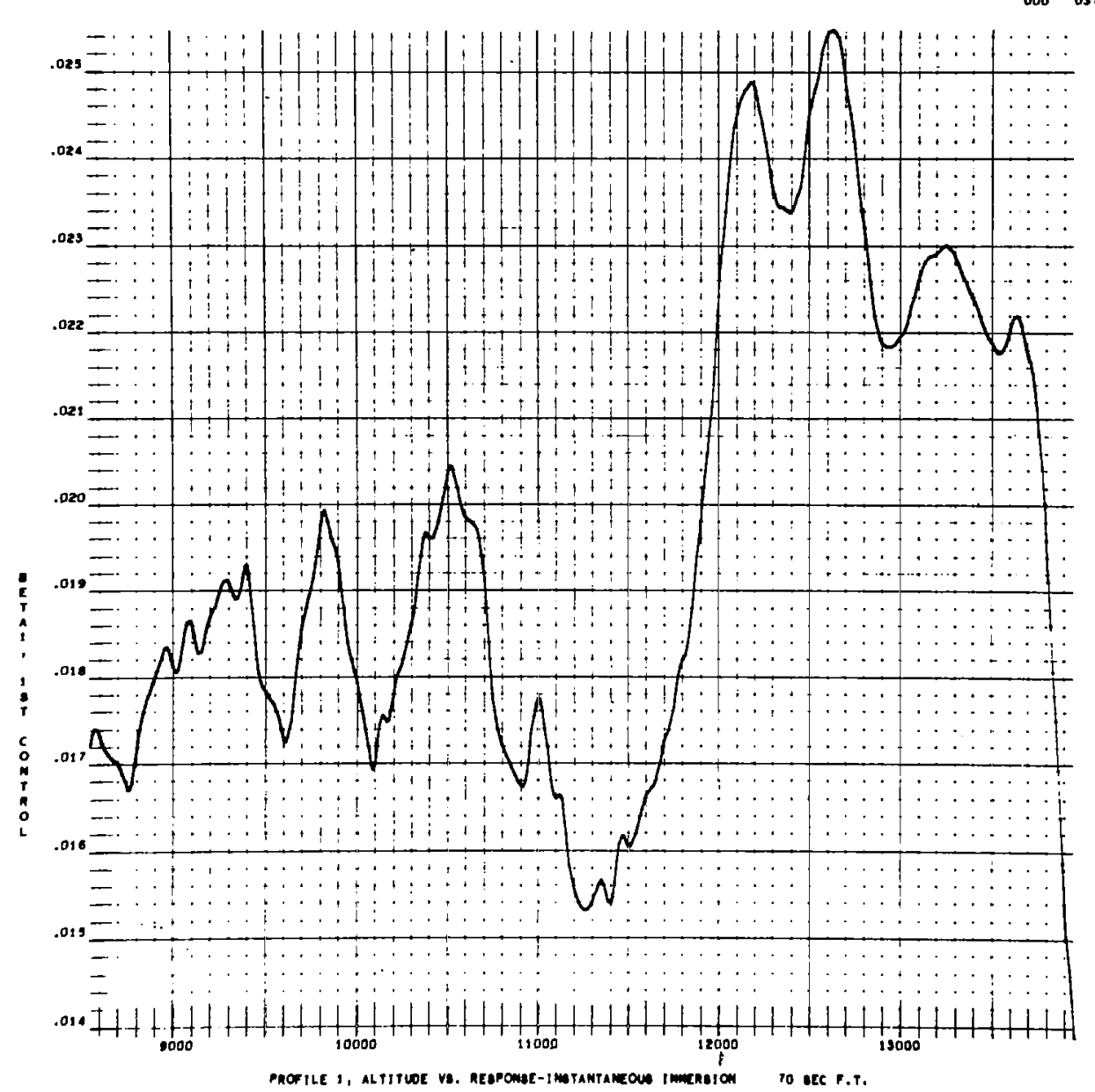

Fig. 31 - Wind-Induced Response of lst Control Considering Instantaneous Immersion - $70 \mathrm{sec}$. F.T. Band 


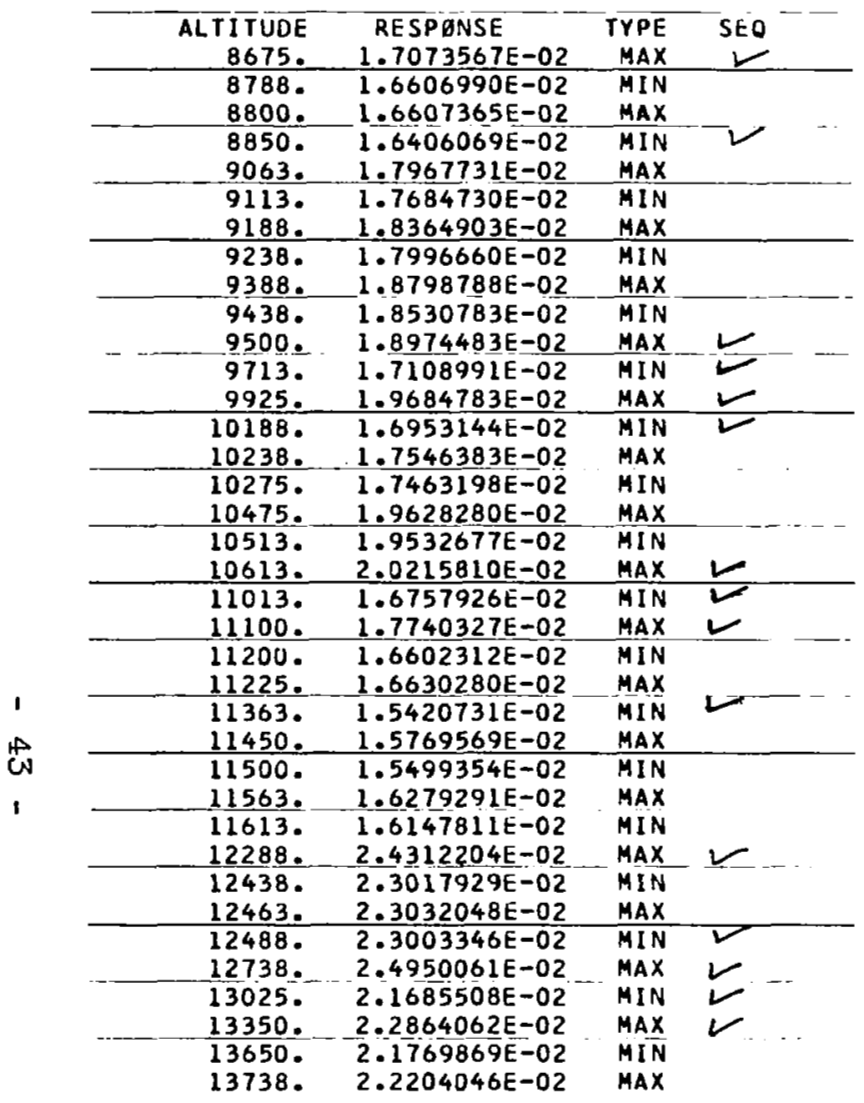

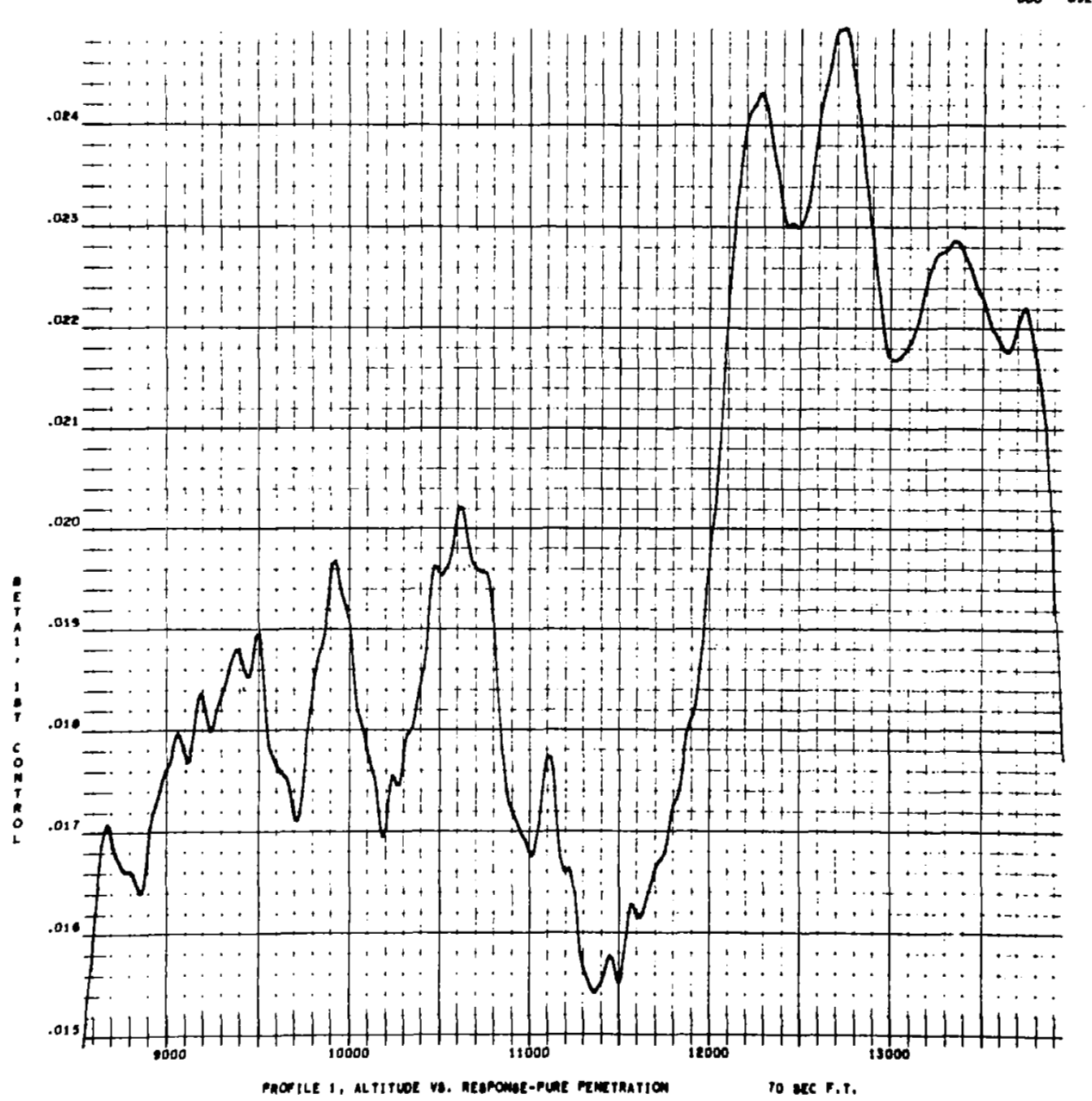

Fig. 32 - Wind-Induced Response of lst Control Considering Pure Penetration - $70 \mathrm{sec}$. F.T. Band 
PRGFILE 1, ALTITUDE VS. RESPQNSE-PENETRATIGN W/ LIFT GRQHTH 70 SEC F.T.

BETAL, IST CONTREL
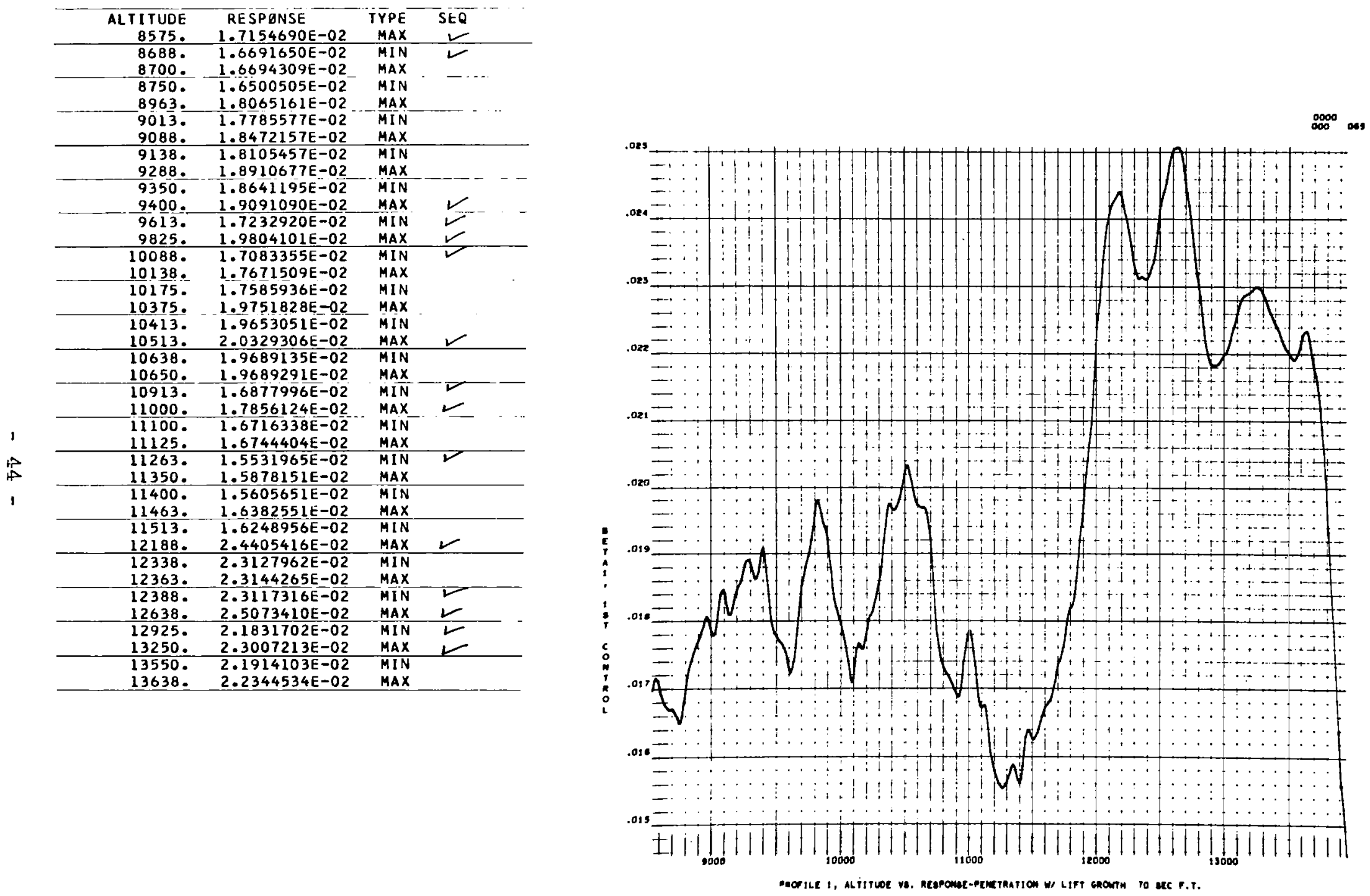

Fig. 33 - Wind-Induced Response of Ist Control Considering Penetration

With Lift Giowth - $70 \mathrm{sec}$. F.T. Band 
The pure penetration and penetration with lift frowth representations yield slightly smaller oscillatory excursions than instantaneous inmersion. These differences are small (not more than 10 per cent of the excursion amplitude or area) and it is difficult to predict the effect on calculated responses due to actual wind profiles. It is anticipated, however, that the aerodynamic represenations which include penetration will provide smaller oscillatory excursions.*

A second feature was expected and verified in the responses to inpulse and step winds. Those responses which exhibit a long, slow approach to their steady-state values are, during this approach, nearly independent of the aerodymamic representation used. This similarity in responses indicates that the local average responses to wind profiles should be nearly independent of the aerodynanic representation used.

The indicial and impulse responses are seen to provide clues for the analysis of wind profile responses. In the wind-induced responses calculated with the three aerodynamic representations, the local averages are expected to be similar while the deviations from the local average will be largest using instantaneous immersion. These ideas about the wind profile induced responses are tested using formal analytical procedures.

Responses are calculated for just two wind profiles. The main problem is how to use this small amount of data for a comparison of the responses from the three aerodymanic representations. The comparisons are made using extremals. The postulated differences in excursions from the local average are tested by comparing the distributions of extreme excursions. The local averages are compared using the average of the envelope of points which in pairs define the extreme excursions.

The following procedure is used for each response in a calculated altitude interval.** The response is examined and those extreme points are selected which appear to lie on or near envelope curves which would enclose

* The reduction in excursions is important because maximum responses very often will be due to excursions or deviations from a "local average." * The altitude intervals vary from 3,510 to 6,375 meters. Normally, constant missile characteristics should probably not be used for intervals which are this large. However, in this case, the additional adjunct vind profile can be considered typical of the altitude region and thus provides additional typical response data. 
the response.* The values of the response at these points are read and then adjunct values are differenced. The magnitudes of these differences are extreme excursions and are analyzed as a sample of extremals from a single population. The analysis of the excursions include ordering, transforming and curve fitting. The transformation used is

$$
y_{m}=-\log _{e}\left(-\log _{e} \Phi_{m}\right)
$$

where $y$ is called the reduced variate, $\Phi_{m}=\frac{m}{n+1}$, the accumulative probability in a sample of $n$ maximums and $m$ is the order from the sinallest $(m=1)$.

The transformation is designed to provide $x_{m l}$ as a linear function of $y_{m}^{* *}$, where $x_{m}$ is the observed $m^{t h}$ ordered maximum in the sample of $n$ maximums. The curve fit is made in the $x, y$ coordinates using the method of least squares.

A local average response is calculated as the unbiased average of the extreme point values initially selected from the response. Then an odd number of points is selected, the number of maximuns and minimums are unequal. This bias is removed with a weight factor of one-half on the first and last points.

The analytical techniques described here constitute unconventional employments of conventional methods. Ordinarily, a sample of extremes is obtained by taking from each of a number of equal-sized samples the largest (or smallest) value. The resulting distribution is used to predict probability of occurrence of an extreme value in an even larger number of similar-sized samples taken from the same population. An associated interpretation provides the return period which is the expected number of equal-sized samples which will be required to locate an assigned maximum.

* Since the responses being compared are very much alike, the near envelope points selected are in all cases equivalent points.

* See "Statistics of Extremes" by E. J. Gumbel, Columbia University Press, or "Statistical Theory of Extreme Values and Some Practical Applications" by E. J. Gumbel, National Bureau of Standards, Applied Mathematics Series 33. The transformation is applicable to maximums from populations with probability functions of the "exponential type." Many important distributions are of this type including the exponential itself, the normal, the chisquare, the logistic, and the log normal. 
In the present analysis, the basic sample size is the altitude increment in which responses are calculated with frozen missile properties. However, not just one maximum excursion is taken from this sample of the calculated response. The number of excursions taken are all those which appear to extend to envelope curves. This selection takes the attitude that the response excursions are a superposition of responses involving the spectral content of the wind and admittance properties of the vehicle. With this viewpoint, the successive "maximum excursions" may be considered extremals in a superposition of independents, * thereby justifying the use of the analysis. It is clear that the conventional connotation of return period has been altered here. It might be possible to recover this predictive capability by reinterpreting sample size. However, this predictive use of the present analysis is not recomnended. Instead, the analysis is recommended for the comparison of excursions obtained using the three aerodynanic representations. The analysis permits the cormarison to be made in an integrated rather than point fashion and uses the majority of pertinent data. The trend with $\mathrm{y}$, the reduced variate, may be interpreted simply as the nonlinear dimension of increasing numbers of samples or increasing numbers of missile transverses.

The calculation of an average from the average of the extremes is not conventional but is an accepted and often powerful technique.

Figure 34 shows an example of the excursion analysis and resulting curve fits plotted on paper designed for this purpose. In the adjacent Fig. 35, the same data are shown on rectilinear coordinates, the observed variate (ordinate) and the reduced variate (abscissa) which is the transformed cumulative probability. The similar curve fits for the analyzed responses are shown on rectangular coordinates in Appendix IV.

* The problem of independence in the primary data arises in the analysis of river flows. There, it is an inferred assumption that the flow during each 24-hr. period is an independent measure, although correlation between the flows of successive days is easily demonstrated. The theory of the extremes is still very successful in this case. 


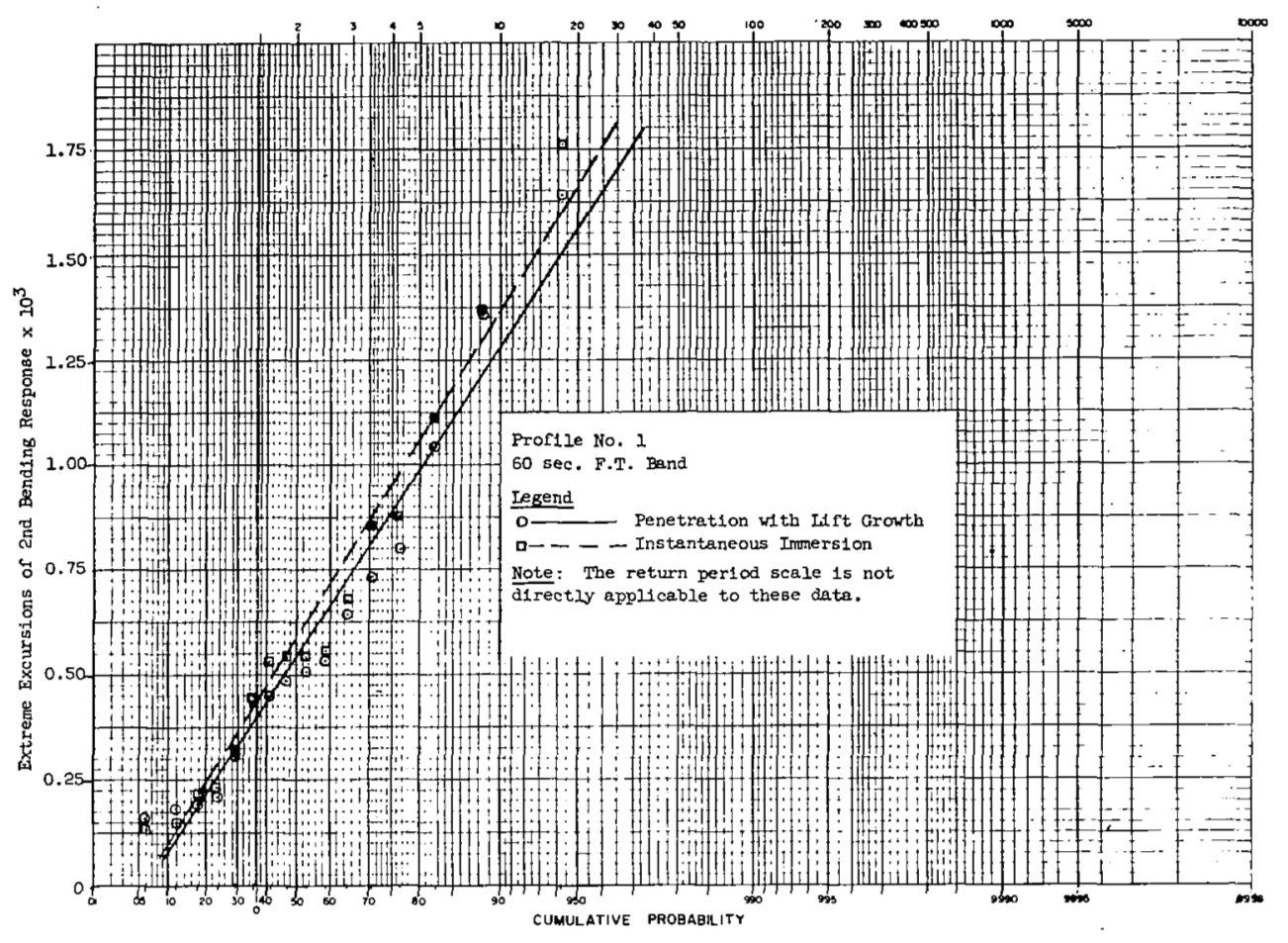

Fig. 34 - Extreme Excursion of and Bending vs. Cumulative Probability - $60 \mathrm{sec}$. F.T.

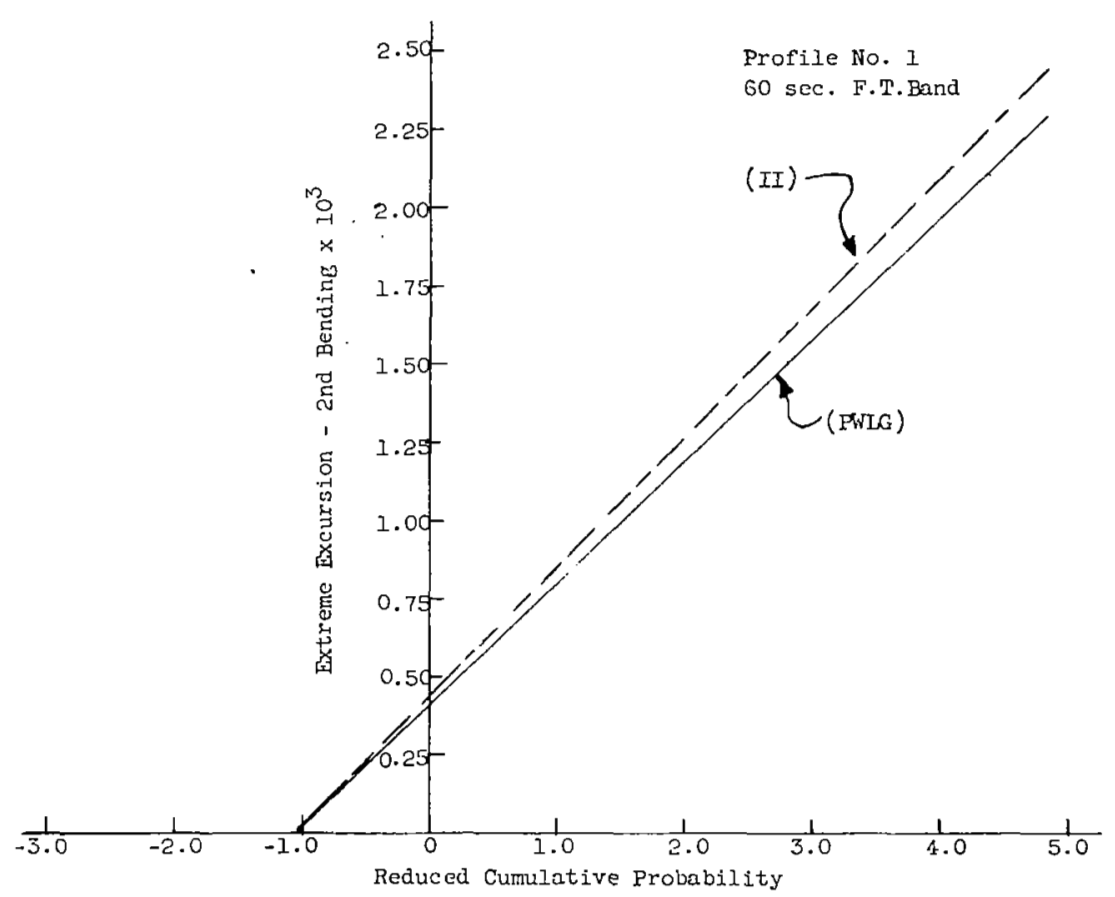

Fig. 35 - Extreme Excursion of 2nd Bending vs. Reduced Cumulative Probability - $60 \mathrm{sec}$. F.T. 


\section{RESULTS AND DISCUSSION}

In this section the results are referenced and discussed not only for their immediate import but also as a guide to future prediction of penetration and lift growth effects.

Samples of the wind-induced responses are shown in Figs. 25 through 33 for the three different aerodynamic representations. The most important result is the small difference between the responses and between the maximum responses. The same result is observed in the comparisons for other responses and flight times. Generally, when the difference in maximum responses exceeds 1 per cent the instantaneous immersion results are conservative (large). The differences in maximum responses with the aerodynamic representations do not appear significant in an engineering sense. For instance, with profile No. 1, a moderately severe profile, instantaneous immersion provides first bending which is conservative by -0.7 per cent to 1.3 per cent. Second bending with instantaneous immersion is conservative by -1.0 per cent to 2.3 per. cent, while the first sloshing response is conservative by -0.8 per cent to 4.8 per cent.

The results and interpretations which follow indicate that the per cent difference may be larger in weak wind profiles but the magnitude of the differences will remain unimportant.

The results indicate that the responses are insignificantly affected by the inclusion of penetration effects and lift growth effects. However, the conclusion can be drawn only for the missile configuration used and the modes included. The possibilities for more general results are explored next.

Samples of the indicial and impulsive responses are shown in Figs. 7 through 24. There is a small but noticeable difference between the responses which do and do not include penetration. The oscillatory excursions with penetration ( $P P$ and $P W L G$ ) are smaller than those from instantaneous immersion. This is especially true for the bending and sloshing modes. It was then anticipated that the same type of difference in oscillatory excursions might appear in the responses to wind profiles. An analysis of the wind-induced excursions was designed and applied as described in Section IV. The results are shown in Figs. 42 through 89 (pp. 109 through 135) where the anticipated differences are observed. In these figures the ordinate is the magnitude of excursions and the abscissa is a nonlinear dimension of increasing transverses or sample sizes.

Figures 42 through 89 illustrate the close correspondence between responses with pure penetration (PP) and penetration with lift growth (PULG). The difference between responses calculated with and without penetration are seen to diminish with altitude. This would be expected since with higher speeds the penetration cases are approaching instantaneous immersion. 
The indicial and impulsive responses (Figs. 7 through 24) also indicate an important similarity in responses with all three aerodynamic representations. This similarity occurs in the long slow approach to steady-state values. The local average values of the vind-induced responses depend largely on this long tall and the history of wind inputs. Thus, local average responses to winds were expected to be about equal for all aerodynamic representations. This expectation is borne out by the averages presented in Figs. 90 through 99 (pp. 136 through 141).

The differences and similarities in the indicial and impulsive responses seem to have their logical counterparts in the wind-induced responses taken as a whole. However, since main interest attaches to the prediction of maximum responses we must test the extension of the same general logic to estimation of maximum responses calculated with the three different aerodymamic representations. The results of this test are shown in Tables III and IV.

TABLE III

RATIOS OF AVERAGE RESPONSES, OBSERVED MAXIMUM RESPONSES AND EXPECTED MAXIMUM RESPONSES FOR PROFILE NO. 1

\begin{tabular}{|c|c|c|c|c|c|c|c|}
\hline \multirow{3}{*}{$\begin{array}{c}\text { Flight } \\
\text { Time } \\
\text { Band } \\
\text { (sec.) }\end{array}$} & \multirow[b]{3}{*}{ Response } & \multicolumn{2}{|c|}{$\begin{array}{c}\text { Avg. Response } \\
\text { Ratios }\end{array}$} & \multicolumn{2}{|c|}{$\begin{array}{c}\text { Observed Max. } \\
\text { Ratios } \\
\end{array}$} & \multicolumn{2}{|c|}{$\begin{array}{l}\text { Expected Max. } \\
\text { Ratios } \\
\end{array}$} \\
\hline & & II & $\mathrm{PP}$ a & $I I$ & $\mathrm{PP}$ & II & $\mathrm{PP}$ \\
\hline & & PWLG & PWLG & PULG & PWLG & PWLG & PWLG \\
\hline & Lst Bending & & & & & & \\
\hline 50 & & 0.9967 & 1.0039 & 1.0092 & 1.0034 & 1.0254 & 1.0036 \\
\hline 60 & & 0.9972 & 1.0028 & 0.9973 & 1.0028 & 1.0122 & 1.0025 \\
\hline 70 & & 1.0024 & 0.9941 & 1.0129 & 0.9954 & 1.0110 & 0.9952 \\
\hline 80 & & 0.9924 & 0.9959 & 1.0082 & 0.9972 & 1.0097 & 0.9989 \\
\hline 90 & & 0.9746 & 1.0020 & 0.9932 & 1.0032 & 1.0054 & 1.0028 \\
\hline & end Ben & & & & & & \\
\hline 50 & & 0.9970 & 1.0049 & 1.0045 & 1.0043 & 1.0362 & 1.0046 \\
\hline 60 & & 0.9917 & 1.0113 & 0.9907 & 1.0113 & 1.0039 & 1.0106 \\
\hline 70 & & 1.0091 & 0.9899 & 1.0178 & 0.9911 & 1.0182 & 0.9910 \\
\hline 80 & & 1.0113 & 0.9786 & 1.0229 & 0.9817 & 1.0236 & 0.9839 \\
\hline 90 & & 0.9737 & 0.9993 & 0.9884 & 0.9999 & 1.0004 & 1.0000 \\
\hline & Lst Sloshing & & & & & & \\
\hline 60 & & 1.0028 & 1.0093 & 0.9921 & 1.0080 & 1.0480 & 1.0054 \\
\hline 70 & & 1.0117 & 0.9869 & 1.0472 & 0.9953 & 1.0376 & 0.9930 \\
\hline 80 & & 1.0204 & 0.9506 & 1.0397 & 0.9926 & 1.0480 & 0.9938 \\
\hline 90 & & 1.0103 & 1.0045 & 1.0458 & 1.0029 & 1.0363 & 1.0026 \\
\hline
\end{tabular}

a/ II denotes instantaneous immersion; PP denotes pure penetration; PWLG denotes penetration with lift growth. 
RATIOS OF AVERAGE RESPONSES, OBSERVED MAXIMUM RESPONSES AND EXPECTED MAXIMUM RESPONSES FOR PROFILE NO. 2

\begin{tabular}{|c|c|c|c|c|c|}
\hline \multirow{3}{*}{$\begin{array}{c}\text { Flight } \\
\text { Time } \\
\text { Band } \\
\text { (sec.) } \\
\end{array}$} & $\begin{array}{l}\text { Avg. Response } \\
\text { Rattos }\end{array}$ & \multicolumn{2}{|c|}{$\begin{array}{l}\text { Observed Max. } \\
\text { Ratios }\end{array}$} & \multicolumn{2}{|c|}{$\begin{array}{c}\text { Expected Max. } \\
\text { Ratios }\end{array}$} \\
\hline & $\mathrm{PP}$ & $I I$ & PP & II & $\mathrm{PP}$ \\
\hline & PWLG & PULG & PWLG & PWLG & PWLG \\
\hline & & & & & \\
\hline 50 & 1.0033 & 1.0669 & 1.0030 & 1.0578 & 1.0029 \\
\hline 60 & 1.0033 & 1.0178 & 1.0024 & 1.0286 & 1.0025 \\
\hline 70 & 0.9945 & 1.0329 & 0.9977 & 1.0283 & 0.9978 \\
\hline 80 & 0.9965 & 1.0140 & 0.9994 & 1.0264 & 1.0017 \\
\hline 90 & 1.0031 & 1.0048 & 1.0033 & 1.0114 & 1.0033 \\
\hline & & & & & \\
\hline 50 & 1.0045 & 1.0747 & 1.0038 & 1.0766 & 1.0041 \\
\hline 60 & 1.0134 & 1.0275 & 1.0104 & 1.0384 & 1.0107 \\
\hline 70 & 0.9900 & 1.0445 & 0.9935 & 1.0418 & 0.9944 \\
\hline 80 & 0.9811 & 1.0432 & 0.9846 & 1.0372 & 0.9889 \\
\hline 90 & 1.0004 & 1.0208 & 1.0006 & 1.0075 & 1.0006 \\
\hline & & & & & \\
\hline 60 & 1.0075 & 1.0987 & 1.0002 & 1.0892 & 1.0008 \\
\hline 70 & 0.9886 & 1.0694 & 0.9987 & 1.0739 & 1.0004 \\
\hline 80 & 0.9577 & 1.0501 & 0.9972 & 1.0528 & 0.9990 \\
\hline 90 & 1.0001 & 1.0179 & 1.0018 & 1.0181 & 1.0020 \\
\hline
\end{tabular}

Here, the penetration with lift growth results are used as a reference since this representation is the most accurate.

The expected values of maximum response used in the ratios of Tables III and IV were formed as the local average plus one-half the expected maximum excursion. Where the expected maximum excursion was the value indicated by the curve fits in Figs. 42 - 89. Comparison of the tabulated ratios for profile No. 1 indicates some correlation between observed and expected values especially for the sloshing response. However, a significant part of the deviations from 1.0 are due to the averages which constitute a sizeable part of the response.

The tabulated ratios for profile No. 2, a weak profile, show excellent agreement between expected and observed ratios.

It appears that the differences and similarities observed in the indicial and impulsive responses can be used with partial success to predict 
the effects of penetration and lift growth. The difference in impulsive excursions appears in wind-induced responses as a change in extreme excursions. Where these excursions play the important role in the maximum response (a weak wind profile) the maximum responses are affected (by penetration primarily) and to about one-half the extent indicated by the comparison of indicial and impulsive responses.

In a moderately severe wind profile the differences in averaged responses are likely to be as important as the oscillatory excursions. The differences in averaged responses are difficult to predict from the comparisons of indicial and impulsive responses. In addition, it appears likely that some intermediate frequencies may play a role of equal significance and be difficult to detect in the comparison of indicial and impulsive responses.

For responses which would be significantly affected by penetration and lift growth it is likely that this fact would be apparent in the comparison of indicial and impulsive responses based on the three aerodynamic representations.

There is a simple and appealing idea which in the past has been employed to speculate about the effects of penetration. This idea is presented here and is shown by comparison with calculated results to be insufficient for predicting the effects of penetration.

The displacements in the bending modes change sign along the length of the missile. The generalized forcing function which drives one of these bending modes takes on different characteristics when penetration is neglected and included.

When penetration is neglected each station of the missile is immersed in the same wind-induced crossflow.* The resulting generalized force for the bending mode is an algebraic sum of local contributions where the changes in mode shape sign lead to cancellations.

* The discussion here pertains only to wind-induced crossflows. The crossflows due to the local transverse body velocities will always provide damping in the analyses reported here. If growth of lift delays were added to the forces from these crossflows the damping might be reduced or eliminated. The crossflows due to the local body angle of attack are $90^{\circ}$ out of phase with body velocity and in the long run neither add nor remove energy from the bending mode. Adding growth of lift delays to the forces from these latter crossflows could provide either damping or undamping. 
When penetration is included the situation is best illustrated by a unit impulse gust. The gust crossflow is applied to successive stations along the missile with delays appropriate for the time required to penetrate the gust. It is apparent here that the energy first added to the mode at the initial penetration may be augmented or canceled during the penetration by subsequent stations. The critical factors are the phase relationships between the modal response and the succession of inputs. The character of previously calculated responses indicated that the critical phase relationships might be estimated.

Each indicial and impulse response has always been dominated by a frequency of the coupled system which lies fairly close to the uncoupled modal frequency. This led to the idea that penetration effects could be estimated by assuming that each bending mode responds primarily as an uncoupled mode and at its natural frequency. The comparison of modal responses with and without penetration would reduce to a comparison of the following forms.

$$
\frac{R_{\eta_{m}}(t)}{C_{\eta_{m}}}=\int_{\tau=0}^{t} Q_{\eta_{m}}(\tau) \sin \omega_{\eta_{m}}(t-\tau) d \tau
$$

and

$$
\begin{aligned}
\frac{r_{\eta_{m}}(t)}{c_{\eta_{m}}} & =\int_{\tau=0}^{L / U} Q_{\eta_{m}}(\tau) d \tau \cdot \int_{\tau=0}^{t} \delta(0) \sin \omega_{\eta_{m}}(t-\tau) d \tau \\
& =\left[\int_{\tau=0}^{L / U} Q_{\eta_{m}}(\tau) d \tau\right] \cdot \sin \omega \eta_{m} t
\end{aligned}
$$

where

$$
\begin{aligned}
& \mathrm{R}_{\eta_{m}}(t)=\begin{array}{c}
\text { The approximated } \mathrm{m}^{\text {th }} \text { bending mode impulse response in- } \\
\text { cluding penetration. }
\end{array} \\
& r_{\eta_{m}}(t)=\text { The approximated response neglecting penetration. } \\
& C_{\eta_{m}}=\text { An unknown admittance amplitude coefficient. }
\end{aligned}
$$




$$
\begin{aligned}
& Q_{\eta_{m}}(\tau)= \text { The growth function for the } \mathrm{m}^{\text {th }} \text { bending mode. (Similar } \\
&\text { to those shown in Figs. } 5 \text { and } 6 .) \\
& \omega_{\eta_{m}}=\text { The uncoupled natural frequency. } \\
& \delta(0)=\text { The unit impulse imposed at time zero. } \\
& \text { sin } \omega_{\eta_{m}} t= \text { The assumed modal response to a unit impulse imposed at } \\
& \text { time zero. }
\end{aligned}
$$

The approximations $R_{\eta_{m}}(t)$ and $r_{n_{m}}(t)$ have been evaluated for the fourth and second bending modes. They are shown in Figs. 36 and 37 . The approximations for the second bending mode response may be compared with the actual, coupled system, impulsive responses shown in Fig. 38.

For both the fourth and second bending modes the approximated responses indicate that incorporation of penetration results in increased response after penetration is completed. During penetration the expected sequence of augmenting and canceling effects are observed.

The approximated second mode responses do not correspond to the coupled system responses. Further, the major implication of the approximated responses (increased response with penetration) is refuted by the responses for the actual coupled system. The main reason for this disagreement appears to lie in strong coupling effects, probably with the control system and swivel engines. These coupling effects raise the predominant frequency of response and provide a significant response to the high frequencies generated during penetration. If the basic idea of the approximation is to be used for the prediction of penetration effects it will be necessary to include some important system coupling.

\section{CONCLUSIONS AND RECCMMENDATIONS}

Specific conclusions about the importance of penetration and lift growth must be restricted to the Saturn C-5 without fins and to the modes included in the analysis. The specific conclusions are:

1. The calculated responses to winds are changed by a detectable but insignificant amount when the aerodynamics are revised to include penetration and lift growth.

2. The change in responses is due primarily to penetration; the addition of lift growth has very little effect. 


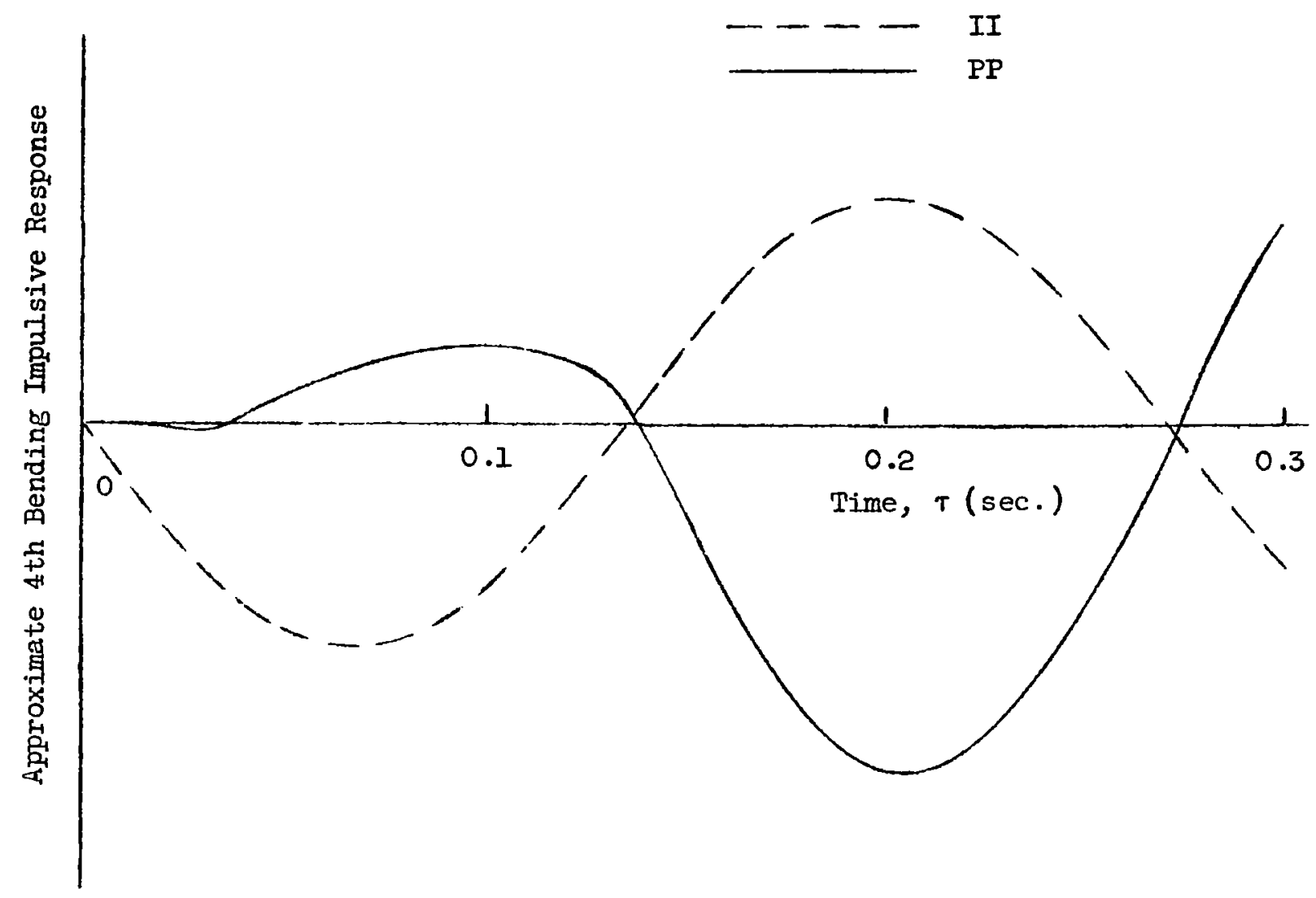

Fig. 36 - Approximate 4th Bending Impulsive Response vs . Response Time - $60 \mathrm{sec}$. F.T. 


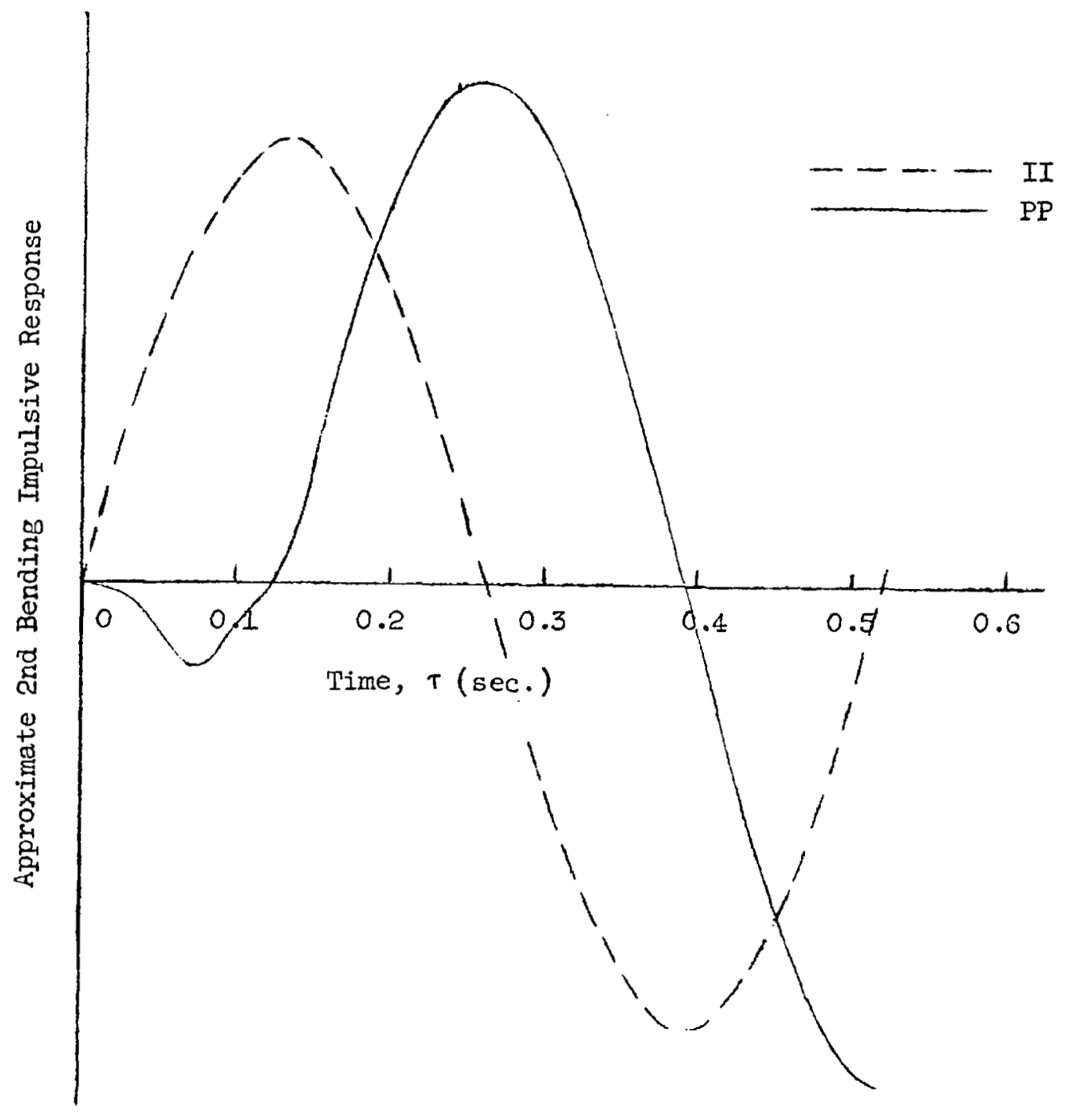

Fig. 37 - Approximate and Bending Impulsive Response vs. Response Time - 60 sec. F.T. 


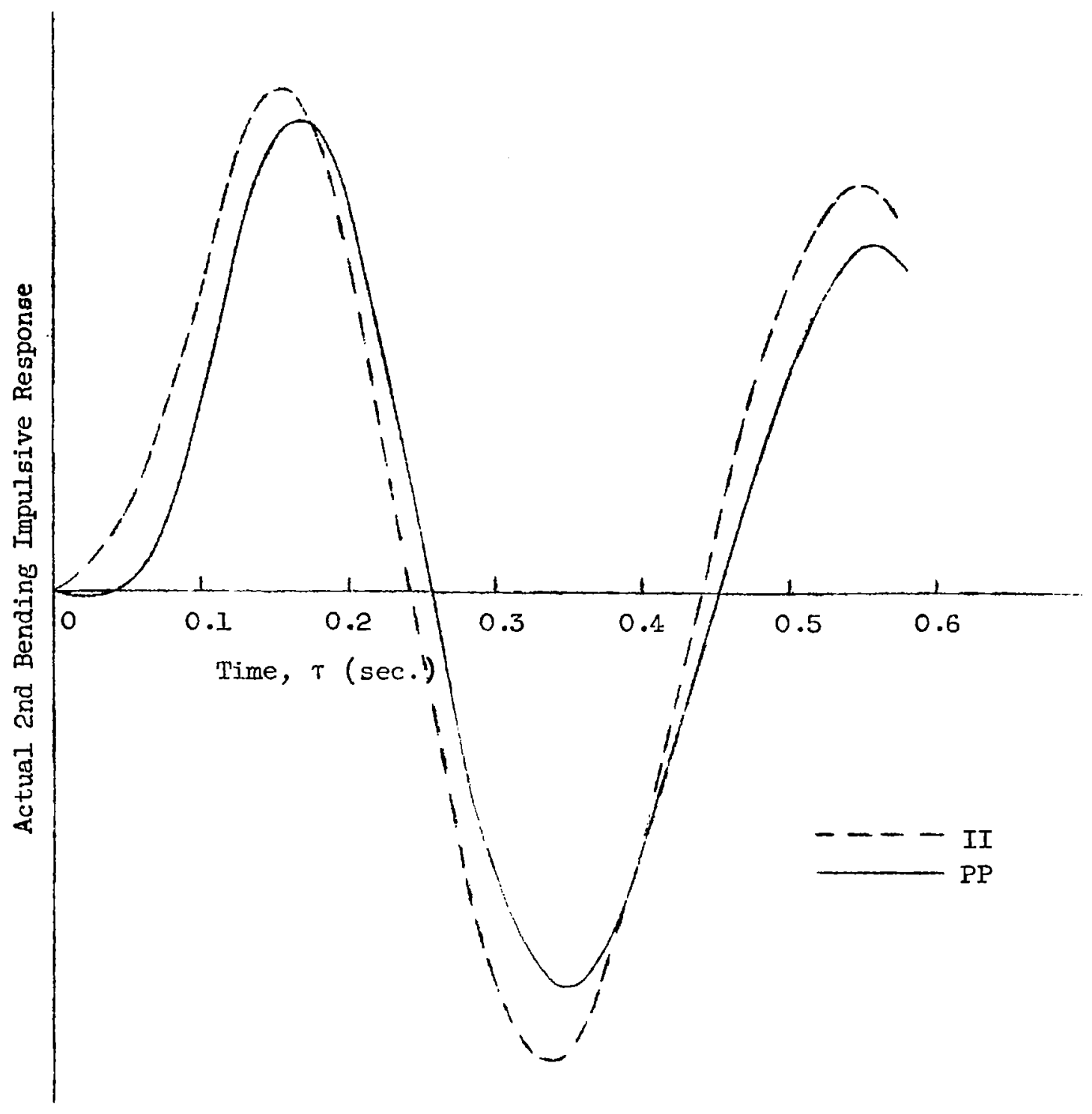

Fig. 38 - Actual 2nd Bending Impulsive Response vs. Response Time - $60 \mathrm{sec}$. F.T. 
3. The maximum responses calculated with instantaneous immersion aerodynamics are conservative (large).

\section{General conclusions are:}

1. The intuitive idea that penetration effects can be predicted from the unccupled bending mode periods and corresponding penetration delays is incorrect.

2. The calculations performed here provide no example of significant effects. However, it appears likely that the cases in which penetration (or penetration and lift grorth) plays an important role can be detected by comparisons of the indicial and impulsive responses using aerodynamics with and without penetration.

It is recommended that:

1. The comparison of indicial and impulsive responses with and without penetration be used as a measure of the adequacy of instantaneous immersion aerodynamics.

2. Comparisons ( 1 above) be carried out for the third and fourth bending modes and for a Saturn C-5 model including fins. (The fins can be simulated by a conic section which provides equivalent rormal forces in the steady state.)

3. The conclusions of this report be checked with a larger amount of wind data by using one or two of the existing impulsive responses to calculate wind responses for a number of profiles in the high $q$ altitude band.

4. In the event a simple predictive technique for penetration and Iift growth effects is sought, consideration should be given to refinement of the isolated mode idea. The refinement would include coupling the mode to the important control frequencies. 


\section{BIBLIOGRAFHY}

1. Yates, J. E., "Transient Aerodymamic Loading on Miltistage Missiles, Midwest Research Institute Phase Report, Project No. 2544-P, March, 1962 (Confidential).

2. Miles, J.W., The Potential Theory of Unsteady Supersonic Flow, Cambridge University Press (1959).

3. Hildebrand, F. B., Introduction to Numerical Analysis, McGraw-Hill Book Company, Inc. (1956).

4. St. John, A. D., and R. R. Blackburn, "Research on the Loading of Missiles Due to Turbulence and Wind Shear," Midwest Research Institute Final Report, Project No. 2544-P, October, 1962 (Confidential).

5. Rheinfurth, M. H., "Control-Feedback Stability Analysis," ABMA Report, DA-TR-2-60.

6. Ward, G. N., Linearized Theory of Steady High-Speed Flow, Cambridge University Press (1955).

7. Luke, Y. L., "A Procedure for the Inversion of a Class of Laplace Transforms," Midwest Research Institute Report, Project No. 2383-P, July, 1961. 
The equations of motion (see [5]) of a flexible missile system in vertical flight are given in this section. Ten generalized coordinates are considered (see Vol. III): lateral translation, $y_{0}$; rotation, $\varnothing$; first bending, $\eta_{1}$; second bending, $\eta_{2}$; third bending $\eta_{3}$; fourth bending, $\eta_{4}$; two sloshing, $\boldsymbol{\zeta}_{1}$ and $\xi_{2}$; actual ending deflection, $\beta_{E} ;$ and control deflection, $B_{C}$ (see Fig. 39 ). The equations are valid for a swivel engine controlled vehicle where the swiveled engines account for four-fifths of the total thrust force. Slender body theory is used to describe the generalized aerodynamic forces.

The oscillating propellants are described by a mechanical analogy (see Fig. 40). Only the motion of the liquid in the booster tanks is investigated. The first sloshing mode is associated with the furthest aft tank (tank A), while the second sloshing mode is associated with the adjacent tank (tank B).

For simplicity, the following terms of the equations of motion (see [5]) are neglected on the basis of being small by comparison:

1. The rotation of the missile cross sections during bending.

2. I corr (the difference of mass moment of inertia of the frozen liquid and liquid propellant in the full tanks about the c.g. of the missile).

3. Generalized forces due to the flowing propellants. (This eliminates the terms containing the time derivatives of the mass of the propellant.)

Assuming that the missile and atmospheric parameters are constant in predetermined time or altitude intervals, the equations of motion become for translation, *

The numerical constant, 4/5, appearing in (I-I), (I-2), and (I-3) as a multiplier of $B_{E}$ can be generalized to account for any percentage of the total number of engines which are swiveled. 


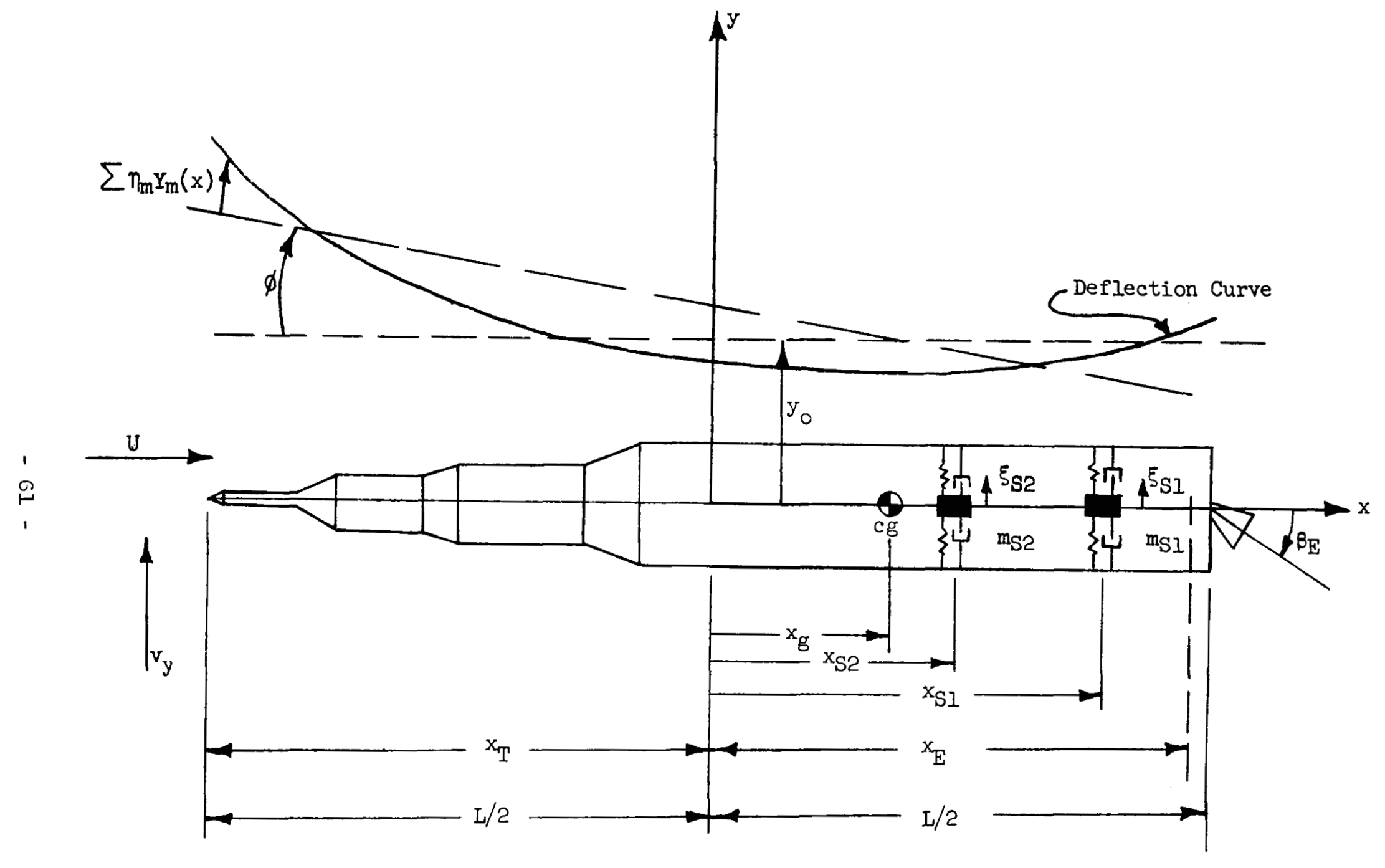

Fig. 39 - Saturn C-5 Coordinate System 


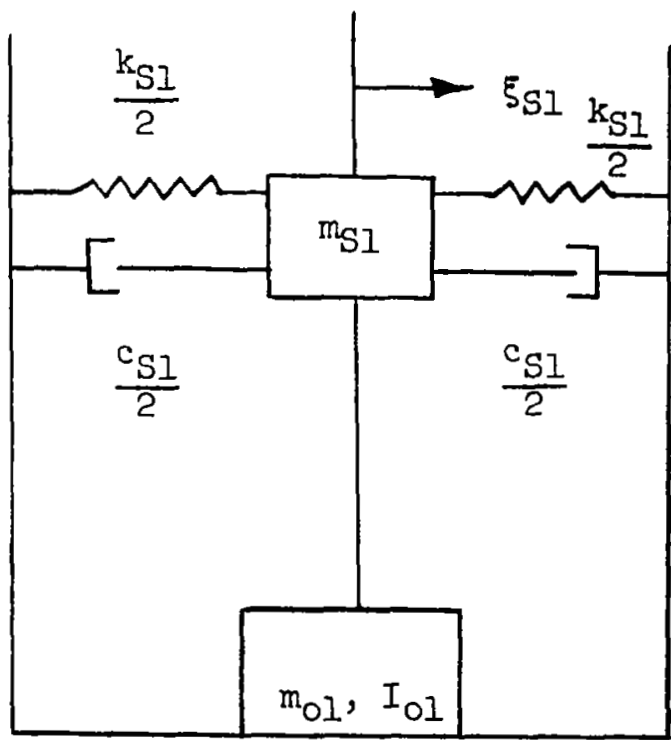

Tank A

$k_{\mathrm{SI}}=\omega_{\mathrm{S} I}^{2} \mathrm{~m}_{\mathrm{SI}}$

$c_{S I}=w_{S I} m_{S I} g_{S I}$

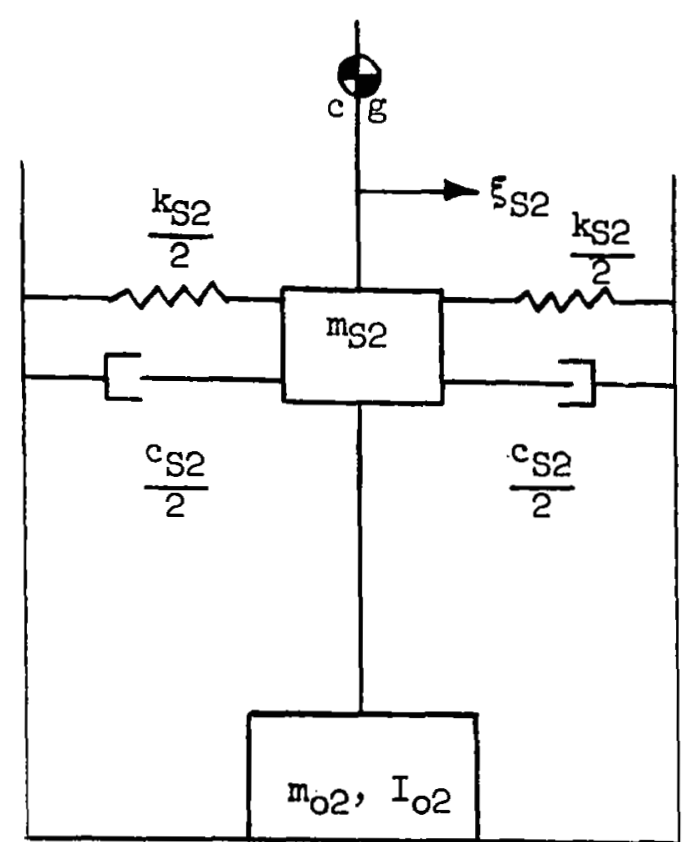

Tank B

$\mathrm{k}_{\mathrm{S} 2}=w_{\mathrm{S} 2 \mathrm{~m}_{\mathrm{S} 2}}^{2}$

$c_{\mathrm{S} 2}=w_{\mathrm{S} 2} m_{\mathrm{S} 2} \mathrm{~g}_{\mathrm{S} 2}$

$\mathrm{m}_{\mathrm{S} 1}, \mathrm{~m}_{\mathrm{S} 2}=$ Sloshing Masses

$\mathrm{m}_{\mathrm{ol}}, \mathrm{m}_{\mathrm{o} 2}=$ Fixed Masses in Sloshing Analogy

$I_{01}, I_{02}=$ Mass Moments of Inertia of Fixed Masses in Sloshing Analogy about Their c.g.

Fig. 40 - Mechenical Analogy of Sloshing Fluids 


$$
\begin{aligned}
& {\left[m+\frac{Q J_{O}}{U^{2}}\right] \ddot{y}_{O}-\frac{Q J_{I}}{U^{2}} \ddot{\phi}} \\
& +\sum_{i=1}^{4}\left\{m_{E} Y_{i}\left(x_{E}\right)+S_{E} Y_{i}^{\prime}\left(x_{E}\right)+\frac{Q G_{i}}{U^{2}}\right\} \ddot{\eta}_{i}+m_{S I} \ddot{\xi}_{I}+m_{S 2} \ddot{\xi}_{2} \\
& -S_{E} \ddot{\beta}_{E}+\frac{2 Q F_{O}}{U} \dot{y}_{O}-\frac{2 Q}{U}\left[F_{I}+J_{O}\right] \dot{\phi}+\sum_{i=1}^{4} \frac{2 Q}{U}\left\{D_{i}+H_{i}\right\} \dot{\eta}_{i} \\
& -\left[F+2 Q F_{O}\right] \phi+\sum_{i=1}^{4}\left\{F Y_{i}^{\prime}\left(x_{E}\right)+Q E_{i}\right\} \eta_{i}-\frac{4}{5} F B_{E}=N(t),
\end{aligned}
$$

for rotation,

$$
\begin{aligned}
& -\frac{Q J_{1}}{U^{2}} \ddot{y}_{O}+\left[I_{c g}+\frac{Q J_{2}}{U^{2}}\right] \ddot{\phi} \\
& -\sum_{i=1}^{4}\left\{\left[S_{E}+m_{E}\left(x_{E}-x_{g}\right)\right] Y_{i}\left(x_{E}\right)+\left[\theta_{E}+S_{E}\left(x_{E}-x_{g}\right)\right] Y_{i}^{\prime}\left(x_{E}\right)+\frac{Q \bar{G}_{i}}{U^{2}}\right\} \ddot{\eta}_{i} \\
& -m_{S 1}\left(x_{S 1}-x_{g}\right) \ddot{\xi}_{1}-m_{S 2}\left(x_{S 2}-x_{g}\right) \ddot{\xi}_{2}+\left[\theta_{E}+S_{E}\left(x_{E}-x_{E}\right)\right] \ddot{\beta}_{E} \\
& -\frac{2 Q F_{1}}{U} \dot{y}_{O}+\frac{2 Q}{U}\left[J_{1}+F_{2}\right] \dot{\phi}-\sum_{i=1}^{4} \frac{2 Q}{U}\left\{\bar{D}_{i}+\bar{H}_{i}\right\} \dot{n}_{i}+2 Q F_{1} \phi \\
& -\sum_{i=1}^{4}\left\{g_{g}\left[m_{E} Y_{i}\left(x_{E}\right)+S_{E} Y_{i}^{\prime}\left(x_{E}\right)\right]+F\left[\left(x_{E}-x_{g}\right) Y_{i}^{\prime}\left(x_{E}\right)-Y_{i}\left(x_{E}\right)\right]-Q \bar{E}_{i}\right\} \eta_{i} \\
& -m_{S 1} g_{g} g_{1}-m_{S 2} g_{G} g_{2}+\left[g_{g} S_{E}+\frac{4}{5}\left(x_{E}-x_{G}\right) F\right] \beta_{E}=M(t),
\end{aligned}
$$

for $m^{\text {th }}$ bending, 


$$
\begin{aligned}
& {\left[m_{E} Y_{m}\left(x_{E}\right)+S_{E} Y_{m}^{\prime}\left(x_{E}\right)+\frac{Q G_{m}}{U^{2}}\right] \ddot{y}_{0}} \\
& -\left\{\left[m_{E}\left(x_{E}-x_{g}\right)+S_{E}\right] Y_{m}\left(x_{E}\right)+\left[\theta_{E}+S_{E}\left(x_{E}-x_{g}\right)\right] Y_{m}^{\prime}\left(x_{E}\right)+\frac{Q \bar{G}_{m}}{u^{2}}\right\} \ddot{\phi} \\
& +T_{m} \ddot{\eta}_{m}+\sum_{i=1}^{4}\left\{\left[S_{E} Y_{m}\left(x_{E}\right)+\theta_{E} Y_{m}^{\prime}\left(x_{E}\right)\right] Y_{i}^{\prime}\left(x_{E}\right)+\left[m_{E} Y_{m}\left(x_{E}\right)+S_{E} Y_{m}^{\prime}\left(x_{E}\right)\right] Y_{i}\left(x_{E}\right)\right. \\
& \left.+\frac{Q C_{m i}}{U^{2}}\right\} \ddot{\pi}_{i}+m_{S 1} Y_{m}\left(x_{S I}\right) \ddot{\xi}_{1}+m_{S 2} Y_{m}\left(x_{S 2}\right) \ddot{\xi}_{2} \\
& -\left[\mathrm{S}_{\mathrm{E}} \mathrm{Y}_{\mathrm{m}}\left(\mathrm{x}_{\mathrm{E}}\right)+e_{\mathrm{E}} \mathrm{Y}_{\mathrm{m}}^{\prime}\left(\mathrm{x}_{\mathrm{E}}\right)\right] \ddot{\beta}_{\mathrm{E}}+\frac{2 Q D_{\mathrm{m}}}{\mathrm{U}} \dot{\mathrm{y}}_{\mathrm{O}}-\frac{2 Q}{\mathrm{U}}\left[\overline{\mathrm{D}}_{\mathrm{m}}+\mathrm{G}_{\mathrm{m}}\right] \dot{\phi}
\end{aligned}
$$

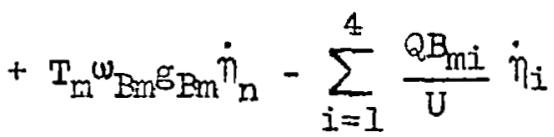

$$
\begin{aligned}
& -\left\{g_{g}\left[m_{E} Y_{m}\left(x_{E}\right)+S_{E} Y_{m}^{\prime}\left(x_{E}\right)\right]+2 Q D_{m}\right\} \phi+T_{m} w_{B m}^{2} n_{m} \\
& +\sum_{i=1}^{4}\left\{g_{g}\left(\left[m_{T} Y_{m}\left(x_{E}\right)+s_{E} Y_{m}^{\prime}\left(x_{E}\right)\right] Y_{i}^{\prime}\left(x_{E}\right)+m_{E} Y_{i}^{\prime}\left(x_{E}\right) Y_{i}\left(x_{E}\right)\right)-Q A_{m i}\right\} \eta_{i} \\
& +m_{S 1} g_{g} Y_{m}^{\prime}\left(x_{S 1}\right) \xi_{1}+m_{S 2} g_{g} Y_{m}^{\prime}\left(x_{S 2}\right) \xi_{2} \\
& -\left[g_{g} S_{E} Y_{m}^{\prime}\left(x_{E}\right)+\frac{4}{5} F Y_{m}\left(x_{E}\right)\right] \beta_{E}=a_{\eta_{m}}(t), \\
& (m=1,2,3,4), \\
& \text { for first sloshing, } \\
& \ddot{y}_{0}-\left(x_{S I}-x_{g}\right) \ddot{\phi}+\sum_{i=1}^{4} Y_{i}\left(x_{S I}\right) \ddot{\eta}_{i}+\ddot{\xi}_{I}+w_{S I} g_{S I} \dot{\xi}_{I}-g_{g} \phi \\
& +\sum_{i=1}^{4} g_{g} Y_{i}^{i}\left(x_{S I}\right) \eta_{1}+\omega_{S I}^{\xi_{I}} \xi_{I}=0
\end{aligned}
$$

- $64-$ 
for second sloshing,

$$
\begin{gathered}
\ddot{y}_{0}-\left(x_{S 2}-x_{g}\right) \ddot{\phi}+\sum_{i=1}^{4} Y_{i}\left(x_{S 2}\right) \ddot{\eta}_{i}+\ddot{\xi}_{2}+w_{S 2 g_{S 2}} \dot{\xi}_{2}-g_{g} \phi \\
+\sum_{i=1}^{4} g_{g} Y_{i}^{\prime}\left(x_{S 2}\right) \eta_{i}+\omega_{S 2}^{2} \xi_{2}=0,
\end{gathered}
$$

for the strivel engine,

$$
\begin{aligned}
& -S_{E} \ddot{y}_{O}+\left[\theta_{E}+s_{E}\left(x_{E}-x_{E}\right)\right] \ddot{\phi}-\sum_{i=1}^{4}\left\{e_{E} Y_{i}^{\prime}\left(x_{E}\right)+S_{E} Y_{i}\left(x_{E}\right)\right\} \ddot{\eta}_{i} \\
& +\theta_{E} \ddot{\beta}_{E}+c_{E} \dot{\beta}_{E}-C_{E} \dot{\beta}_{C}+g_{G} S_{E} \phi-\sum_{i=1}^{4} g_{E} S_{E} Y_{i}^{\prime}\left(x_{E}\right) \eta_{i} \\
& +\left[k_{E}+g_{G} S_{E}\right] \beta_{E}-k_{E} \beta_{C}=0
\end{aligned}
$$

The last equation of motion describes the control and actuator system of the missile. The control system considered in this report utilizes both an attitude reference control and a flow direction indicator (see [5]). The differential equation describing the relationship between the control deflection, $\beta_{C}$, the indicated attitude, $\phi_{i}$, and the indicated angle of attack, $a_{i}$, is given by

$$
\begin{gathered}
\bar{a}_{6} P_{C}^{V I}+\bar{a}_{5} \beta_{C}+\bar{a}_{4} \beta_{C}^{I V}+\bar{a}_{3} \ddot{\beta}_{C}+\bar{a}_{2} \ddot{\beta}_{C}+\bar{a}_{1} \dot{\beta}_{C} \\
+\bar{a}_{0} \beta_{C}=a_{0} \phi_{i}+a_{1} \dot{\phi}_{i}+b_{0} \alpha_{i}
\end{gathered}
$$

where

$$
\phi_{i}=\phi-\sum_{i=1}^{4} y_{i}^{\prime}\left(x_{\phi}\right) \eta_{i}
$$




$$
\begin{aligned}
\sigma_{i} & =-\frac{\dot{y}_{0}}{U}+\frac{\left(x_{v}-x_{g}\right)}{U} \dot{\phi}-\sum_{i=1}^{4} \frac{Y_{i}\left(x_{v}\right)}{U} \dot{\eta}_{i}+\phi \\
& -\sum_{i=1}^{4} Y_{i}\left(x_{v}\right) \eta_{i}+\alpha_{w} .
\end{aligned}
$$

and

$$
\alpha_{\mathrm{w}}=\frac{\mathrm{v}_{\mathrm{y}}}{\mathrm{U}}
$$

The quantities $\bar{a}$ 's in $(I-7)$ are time-independent coefficients while the gain values $a_{0}, a_{1}$ and $b_{0}$ are time-dependent variables.

The terms $N(t), M(t), Q \eta_{2}(t), Q_{\eta_{2}}(t), Q Q_{\eta_{3}}(t)$ and $Q_{\eta_{4}}(t)$ are generalized aerodynamic forces which are functions of the cross flow gust velocity, $v_{y}$ (see Appendix II).

It was convenient for the investigation of the effects and importance of penetration and growth of lift on missile response to simplify the above system of equations in anticipation of generating a voluminous amount of numerical results. Thus, for the study presented in this report the following additional conditions were imposed on $(I-I)$ through (I-9):

1. The third and fourth bending mode contributions were neglected.

2. The compliance of the swivel engine (difference between the actual deflection angle, $\beta_{E}$, and the control signal, $\beta_{C}$ ) was assumed to be zero. This, in essence, is similar to making the linkage connection between the two variables infinitely rigid.

3. The mass of the swivel engine and the mass moment of inertia of the swivel engine about its swivel point were neglected.

Incorporating the above conditions into the above equations yielded for translation, 


$$
\begin{aligned}
& {\left[m+\frac{Q J_{0}}{U^{2}}\right] \ddot{y}_{0}-\frac{Q J_{I}}{U^{2}} \ddot{\phi}+\frac{Q G_{1}}{U^{2}} \ddot{\eta}_{1}+\frac{Q G_{2}}{U^{2}} \ddot{\eta}_{2}} \\
& \quad+m_{S I} \ddot{\xi}_{1}+m_{S 2} \ddot{\xi}_{2}+\frac{2 Q F_{O}}{U} \dot{y}_{0}-\frac{2 Q}{U}\left[F_{1}+J_{0}\right] \dot{\phi} \\
& \quad+\frac{2 Q}{U}\left[D_{1}+H_{1}\right] \dot{\eta}_{I}+\frac{2 Q}{U}\left[D_{2}+H_{2}\right] \dot{\eta}_{2}-\left[F+2 Q F_{0}\right] \phi \\
& \quad+\left[F Y_{1}^{\prime}\left(x_{E}\right)+Q E_{1}\right] \eta_{I}+\left[F Y_{2}^{\prime}\left(x_{E}\right)+Q E_{2}\right] \eta_{2}-\frac{4}{5} F B_{C}=N(t),
\end{aligned}
$$

for rotation,

$$
\begin{aligned}
& -\frac{Q J_{1}}{U^{2}} \ddot{y}_{0}+\left[I_{C g}+\frac{Q J_{2}}{U^{2}}\right] \ddot{\phi}-\frac{Q \bar{G}_{1}}{U^{2}} \ddot{\eta}_{1}-\frac{Q \bar{G}_{2}}{U^{2}} \ddot{n}_{2} \\
& -m_{S 1}\left(x_{S 1}-x_{G}\right) \ddot{\xi}_{1}-m_{S 2}\left(x_{S 2}-x_{g}\right) \ddot{s}_{2}-\frac{2 Q F_{1}}{U} \dot{y}_{0}+\frac{2 Q}{U}\left[J_{I}+F_{2}\right] \dot{\phi} \\
& -\frac{2 Q}{\mathrm{U}}\left[\overline{\mathrm{D}}_{1}+\overline{\mathrm{H}}_{1}\right] \dot{\vec{n}}_{1}-\frac{2 Q}{\mathrm{U}}\left[\overline{\mathrm{D}}_{2}+\overline{\mathrm{H}}_{2}\right] \dot{\mathrm{n}}_{2}+2 Q \mathrm{~F}_{1} \phi \\
& -\left\{F\left[\left(x_{E}-x_{G}\right) Y_{1}^{\prime}\left(x_{E}\right)-Y_{I}\left(x_{E}\right)\right]-Q \bar{E}_{1}\right\} \eta_{I} \\
& -\left\{F\left[\left(\mathrm{x}_{\mathrm{E}}-\mathrm{x}_{\mathrm{g}}\right) \mathrm{Y}_{2}^{\prime}\left(\mathrm{x}_{\mathrm{E}}\right)-\mathrm{Y}_{2}\left(\mathrm{x}_{\mathrm{E}}\right)\right]-Q \overline{\mathrm{E}}_{2}\right\} \eta_{2}-\mathrm{m}_{\mathrm{SI}} \mathrm{g}_{\mathrm{g}} \xi_{I}-\mathrm{m}_{\mathrm{S} 2} \mathrm{~g}_{\mathrm{g}} \bar{\xi}_{2} \\
& +\frac{4}{5}\left(x_{E}-x_{G}\right) F \beta_{C}=M(t) \text {, }
\end{aligned}
$$

for first bending,

- 67 - 


$$
\begin{aligned}
& \frac{Q G_{1}}{U^{2}} \ddot{y}_{0}-\frac{Q \bar{G}_{1}}{U^{2}} \ddot{\phi}+\left[T_{1}+\frac{Q C_{11}}{U^{2}}\right] \ddot{n}_{1}+\frac{Q C_{12}}{U^{2}} \ddot{n}_{2} \\
& +m_{S I} Y_{1}\left(x_{S I}\right) \ddot{\xi}_{1}+m_{S 2} Y_{1}\left(x_{S 2}\right) \ddot{\xi}_{2}+\frac{2 Q D_{1}}{U} \dot{y}_{0}-\frac{2 Q}{U}\left[\bar{D}_{1}+\bar{G}_{1}\right] \dot{\phi} \\
& +\left[\mathrm{T}_{1} \omega_{B I} g_{B I}-\frac{Q B_{11}}{U}\right] \dot{\eta}_{1}-\frac{Q B_{12}}{U} \dot{\eta}_{2}-2 Q D_{1} \phi \\
& +\left[T_{1} \omega_{B 1}^{2}-Q A_{11}\right] \eta_{1}-Q A_{12} \eta_{2}+m_{S 1} E_{S} Y_{1}^{\prime}\left(x_{S 1}\right) \xi_{1} \\
& +m_{S 2} g_{g} Y_{1}^{\prime}\left(x_{S 2}\right) \xi_{2}-\frac{4}{5} F Y_{1}\left(x_{E}\right) \beta_{C}=a_{\eta_{I}}(t) \text {, }
\end{aligned}
$$

for second bending,

$$
\begin{aligned}
& \frac{Q G_{2}}{U^{2}} \ddot{y}_{0}-\frac{Q \bar{G}_{2}}{U^{2}} \ddot{\phi}+\frac{Q C_{21}}{U^{2}} \ddot{n}_{I}+\left[T_{2}+\frac{Q C_{22}}{U^{2}}\right] \ddot{\eta}_{2} \\
& +m_{S I} Y_{2}\left(x_{S I}\right) \ddot{\xi}_{1}+m_{S 2} Y_{2}\left(x_{S 2}\right) \ddot{\xi}_{2}+\frac{2 @ D_{2}}{U} \dot{y}_{0}-\frac{2 Q}{U}\left[\ddot{D}_{2}+G_{2}\right] \dot{\phi} \\
& -\frac{Q B_{21}}{U} \dot{\eta}_{1}+\left[T_{2}{ }^{{ }^{3}}{ }_{B 2} B_{B 2}-\frac{Q B_{22}}{U}\right] \dot{r}_{i 2}-2 Q D_{2} \phi-Q A_{21} \eta_{1}
\end{aligned}
$$

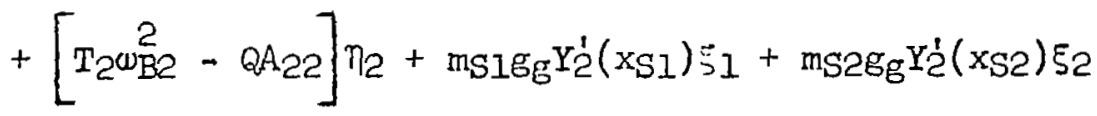

$$
\begin{aligned}
& -\frac{4}{5} F Y_{2}\left(x_{E}\right) \beta_{C}=a \pi_{2}(t) \text {, }
\end{aligned}
$$

for first sloshing,

$$
\begin{gathered}
\ddot{y}_{O}-\left(x_{S I}-x_{g}\right) \ddot{\phi}+Y_{I}\left(x_{S I}\right) \ddot{\eta}_{I}+x_{2}\left(x_{S I}\right) \ddot{\eta}_{2}+\ddot{\xi}_{I} \\
+\omega_{S I} g_{S I} \dot{\xi}_{I}-g_{g} \phi+G_{g} Y_{I}^{\prime}\left(x_{S I}\right) \eta_{I}+g_{g} Y_{2}^{\prime}\left(x_{S I}\right) \eta_{2} \\
+\omega_{S I}^{2} \xi_{I}=0, \\
-68-
\end{gathered}
$$


for second sloshing,

$$
\begin{gathered}
\ddot{y}_{0}-\left(x_{S 2}-x_{g}\right) \ddot{\phi}+Y_{1}\left(x_{S 2}\right) \ddot{\eta}_{I}+Y_{2}\left(x_{S 2}\right) \ddot{\eta}_{2}+\ddot{\xi}_{2}+w_{S 2} g_{S 2} \dot{\xi}_{2} \\
-g_{g} \phi+g_{g} Y_{1}^{\prime}\left(x_{S 2}\right) \eta_{1}+g_{g} Y_{2}^{\prime}\left(x_{S 2}\right) \eta_{2}+\omega_{S 2}^{2} \xi_{2}=0 .
\end{gathered}
$$

Substituting $(I-8),(I-9)$ and $(I-10)$ into $(I-7)$ and reducing to a set of second order equations gives for the control system,

$$
\begin{aligned}
\bar{a}_{6} \ddot{\mathrm{P}} & +\frac{b_{0}}{U} \dot{y}_{0}-\left[a_{I}+\frac{b_{0}\left(x_{v}-x_{g}\right)}{U}\right] \dot{\phi} \\
& +\left[a_{1} Y_{I}^{\prime}\left(x_{\phi}\right)+\frac{b_{0} Y_{I}\left(x_{v}\right)}{U}\right] \dot{\eta}_{I}+\left[a_{I} Y_{2}^{\prime}\left(x_{\phi}\right)+\frac{b_{0} Y_{2}\left(x_{v}\right)}{U}\right] \dot{\eta}_{2} \\
& +\bar{a}_{1} \dot{\beta}_{C}+\bar{a}_{3} \dot{N}+\bar{a}_{5} \dot{P}-\left(a_{0}+b_{0}\right) \phi \\
& +\left[a_{0} Y_{I}^{\prime}\left(x_{\phi}\right)+b_{0} Y_{I}^{\prime}\left(x_{v}\right)\right] \eta_{I}+\left[a_{0} Y_{2}^{\prime}\left(x_{\phi}\right)+b_{0} Y_{2}^{\prime}\left(x_{v}\right)\right] \eta_{2} \\
& +\bar{a}_{0} \beta_{C}+\bar{a}_{2} N+\bar{a}_{4} P=\frac{b_{0} v_{y}}{U}
\end{aligned}
$$

where

$$
N-\ddot{\beta}_{C}=0
$$

and

$$
P-\ddot{N}=0
$$

The preceding equations of motion $((I-11)$ through $(I-19)$ ) describe the missile system considered in the numerical investigation of the effects and importance of penetration and growth of lift on missile response (see Vol. III). 
DEVELOFMENT OF TRANSIENT, QUASI-STEADY AND STEADY GENERAIIZED

AERODYNAMIC FORCE EXPRESSIONS RESUITING FROM A UNIT STEP

AND UNIT TMPULSE WIND PROFIIE

The development of the transient, quasi-steady and steady generalized aerodynamic force expressions resulting from a unit step and impulse wind profile is presented in this Appendix. The development is based on slender body theory. For simplicity, the details of the analysis are omitted and the reader is referred to the original work of Miles [2] and the extension to multi-staged vehicles by Yates [I]. for additional information.

Transient aerodynamic force expressions corresponding to rigid body and bending modes of vibration are presented first for a general wind profile. The indicial* and impulsive** transient, quasi-steady and steady force expressions are then derived. Quadratic polymomials are used to curve fit segments of the mode shapes in evaluating the generalized forces associated with the bending modes.

The wind induced forcing functions which are compared in this report have both a geometric and an aerodynamic aspect.

In the geometric consideration two cases are used. In the simplest case, instantaneous immersion, all stations along the missile are assumed to be immersed in the same wind-induced crossflow, namely the wind crossflow cccurring at the nose. The more accurate geometric representation, called penetration, assigns to each station along the missile the wind crossflow which exists at the altitude occupied by the station. With penetration, the missile nose enters a side gust first and in subsequent time successive stations along the missile length move into the crossflow.

Two representations of the aerodynamics are used; they are quasisteady and transient. In the quasi-steady representation the air forces at a missile station are those which would exist if the local crossflow persisted unchanged for an extended time. The transient representation is based on transient slender body theory and includes the growth of lift with time.

* Response to a unit step wind profile.

* Response to a unit impulse wind profile. 
Three types of wind-induced forcing functions are assembled using combinations of the geometric and aerodynamic representations. The simplest type is called instantaneous-immersion and uses the instantaneous-immersion geometric representation with quasi-steady aerodynamics. A more accurate type, called pure-penetration, uses penetration geometrics and quasi-steady aerodynamics. The most accurate forcing functions are called penetration-with-liftgrowth. These functions use the penetration geometrics with transient aerodynamics .*

The simpler function types, instantaneous-immersion and pure-penetration, can be obtained from penetration-vith-lift-growth which is derived first.

I. TRANSIENT AERODYNAMIC FORCES WITH PENETRATION AND IIFT GROWTH FROM A GENERAL CROSSFLOW VEIOCITY

Consider the multi-staged pointed body of revolution as shown in Fig. 41. The cartesian orthogonal coordinate system $(x-y-z)$ has its origin at the nose. The vehicle is considered to be traveling in the negative $x$ direction with a constant velocity, $U$. At time zero, the nose encounters a side wind of magnitude $v(x, t)$ directed along the positive $z$ axis. Now, from [1] and [2] the transient aerodynamic forces corresponding to translational, rotational and bending coordinates are given, respectively, by

$$
\begin{aligned}
N(t) & =q \int_{0}^{(U t)}\left(\frac{S^{\prime}(x) v\left(t-\frac{x}{U}\right)}{U}\right. \\
& \left.+\frac{2 S^{\prime}(x)}{\operatorname{MR}(x)} \int_{0}^{t-\frac{x}{U}} f_{0}^{\prime}\left[\frac{U\left(t-\frac{x}{U}-\bar{\tau}\right)}{M R(x)}\right] v(\bar{\tau}) d \bar{\tau}\right\} d x,
\end{aligned}
$$

* The crossflows induced by missile motions are in all cases treated with quasi-steady aerodynamics. These crossflows are small in comparison to the wind-induced crossflows and appear in the left hand side of the equations of motion. 


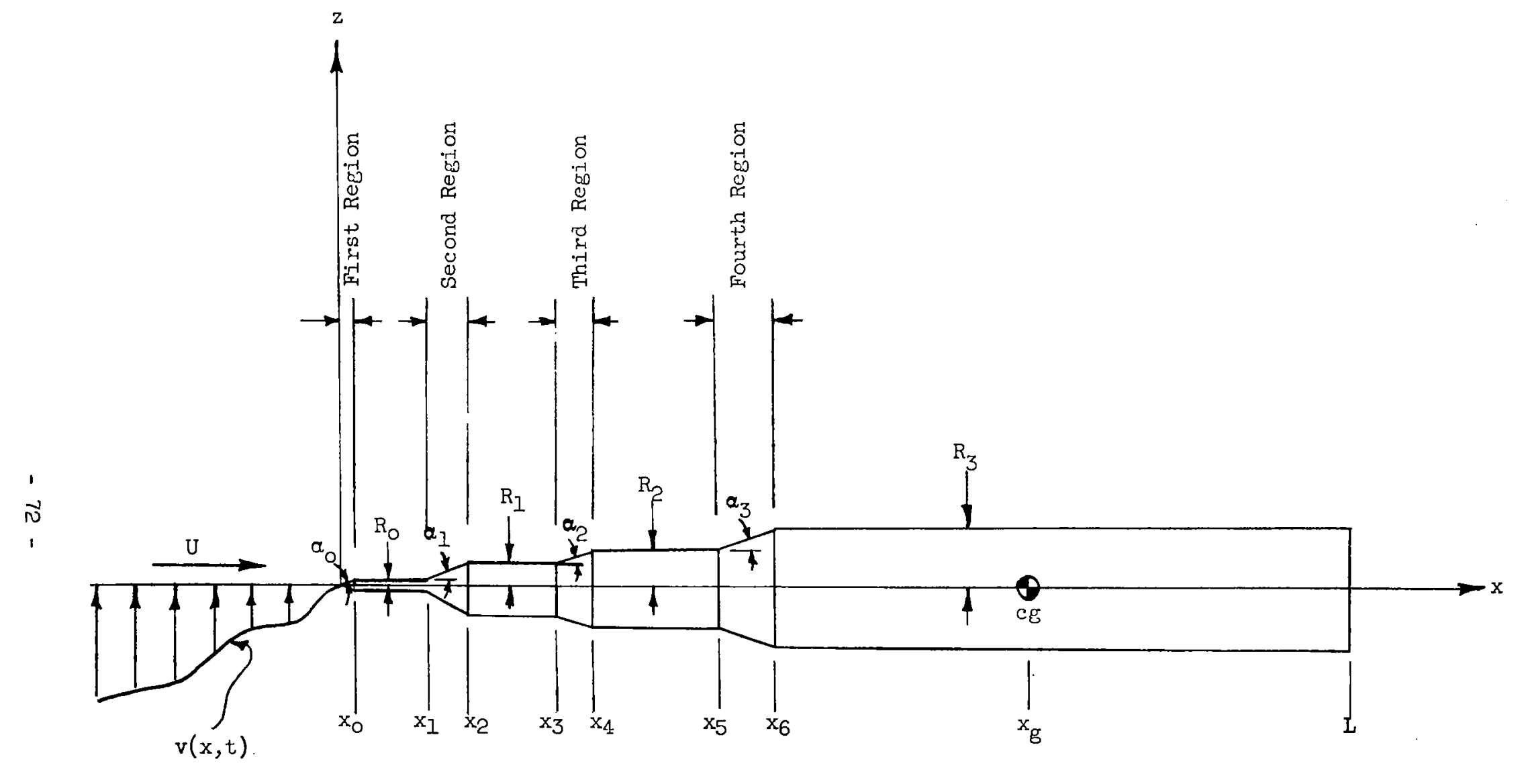

Fig. 41 - Saturn C-5 Body Geometry 


$$
\begin{aligned}
M(t) & =-q \int_{0}^{\left(\begin{array}{c}
U t \\
I
\end{array}\right)}\left(x-x_{g}\right)\left\{\frac{S^{\prime}(x) v\left(t-\frac{x}{U}\right)}{U}\right. \\
& \left.+\frac{2 S^{\prime}(x)}{M R(x)} \int_{0}^{t-\frac{x}{U}} f_{0}^{\prime}\left[\frac{U\left(t-\frac{x}{U}-\bar{T}\right)}{M R(x)}\right] v(T) d \bar{T}\right\} d x,
\end{aligned}
$$

and

$$
\begin{aligned}
Q_{T_{m}}(t) & =q \int_{0}^{\left(\begin{array}{c}
U t \\
L
\end{array}\right)} Y_{m}(x)\left\{\frac{S^{\prime}(x) v\left(t-\frac{x}{U}\right)}{U}\right. \\
& \left.+\frac{2 S^{\prime}(x)}{M R(x)} \int_{0}^{t-\frac{x}{U}} f_{0}^{\prime}\left[\frac{U\left(t-\frac{x}{U}-\bar{\tau}\right)}{\operatorname{MR}(x)}\right] v(\bar{\tau}) d \bar{\tau}\right\} d x
\end{aligned}
$$

where the notation ( $\left(\begin{array}{l}U t \\ \mathrm{~L}\end{array}\right)$ means that the $\mathrm{x}$ integration is performed over $(0, U t)$ or $(O, L)$ as the body has partially or totally penetrated the side wind profile $v\left(t-\frac{x}{U}\right)$. Here $x_{g}$ is the distance from the nose to the center of gravity and the sign of the moment equation is chosen so that positive $M(t)$ gives rise to a clocliwise rotation of the vehicle as viewed in Fig. 41 . In (II-I), (II-2) and (II-3), primes denote total differentiation of the funct1on with respect to its argument.

The exact expression for $f_{0}(\tau)$ (see [1]) is rather cumbersome to work with in obtaining numerical results. A numerical evaluation of this function is given by various authors as are their expansions for large and small arguments (see [2] and [6]). A convenient approximation of this function, valid for all values of the argument, is given by Luke [7]. The approximate mathematical form of $f_{o}(\tau)$ is 


$$
\begin{aligned}
f_{0}(\tau) & =1-0.0405 e^{-0.21005 \tau}-2.7077 e^{-\tau}+0.0016 e^{-1.3063 \tau} \\
& -0.0001 \tau e^{-0.21005 \tau}-0.3920 \tau e^{-\tau} \\
& -\tau e^{-0.61682 \tau}(1.0204 \cos 0.40731 \tau-0.6574 \sin 0.4073 \tau) \\
& +e^{-0.61682 \tau}(2.2466 \cos 0.4073 \tau+0.2066 \sin 0.4073 \tau) \cdot \quad \text { (II-4) }
\end{aligned}
$$

This representation is used in the numerics and computer program presented in this report.

\section{TRANSIENT AERODYNAMIC FORCES FOR SATURN C-5 CONFIGURATION* ENCOUNTERING A UNIT STEP AID UNIT IMPUISE WIND PROFIIE}

\section{A. Unit Step and Unit Impulse Input}

For the special case of a unit step and unit impulse wind profile, the generalized forces corresponding to translational, rotational and bending coordinates reduce to a relatively simple form. We will first consider the indicial transient aerodynamic forces.

\section{Iet}

$$
v(\bar{T})=I(\bar{T}),
$$

where I denotes the Heaviside step function. Substituting (II-5) into (II-I), (II-2) and (II-3), the indicial transient normal force, moment and bending moment become

* The Saturn C-5 configuration considered in this report does not include fins. 


$$
\begin{gathered}
\mathbb{N}_{S}(\tau)=\frac{2 q}{U} \int_{0}^{\left(\begin{array}{l}
U \tau \\
L
\end{array}\right)} S^{\prime}(x) f_{0}\left\{\frac{U\left(\tau-\frac{x}{U}\right)}{M R(x)}\right\} d x, \\
M_{S}(\tau)=-\frac{2 q}{U} \int_{0}^{\left(\begin{array}{l}
U \tau \\
L
\end{array}\right)}\left(x-x_{\tilde{G}}\right) S^{2}(x) f_{0}\left\{\frac{U\left(\tau-\frac{x}{U}\right)}{\operatorname{MR}(x)}\right\} d x,
\end{gathered}
$$

and

$$
Q_{\eta_{m}}(\tau)_{S}=\frac{2 q}{U} \int_{0}^{\left(\begin{array}{l}
U \tau \\
I
\end{array}\right)} Y_{m}(x) S^{\prime}(x) f_{0}\left\{\frac{U\left(\tau-\frac{x}{U}\right)}{M R(x)}\right\} d x
$$

where the subscript $s$ denotes that these entities are due to a unit step wind profile.

If we consider the unit impulse wind profile

$$
v(\bar{\tau})=\delta(\bar{\tau}),
$$

where $\delta(\bar{\tau})$ is the Dirac delta function, the impulsive transient normal force, moment and bending moment become

$$
N_{i}(\tau)=q\left\{\frac{2}{M} \int_{0}^{\left(\begin{array}{l}
U T \\
L
\end{array}\right)} \frac{S^{\prime}(x)}{R(x)} f_{0}^{1}\left\{\frac{U\left(\tau-\frac{x}{U}\right)}{M R(x)}\right\} d x+S^{\prime}(U \tau)\right\}
$$

where

$$
\begin{aligned}
S^{\prime}(U \tau) & =\left.S^{\prime}(x)\right|_{X=U \tau} \text { for } 0 \leq U T \leq L \\
& =0 \text { for } U \tau>I
\end{aligned}
$$




$$
\begin{aligned}
M_{i}(\tau) & =-q\left\{\frac{2}{M} \int_{0}^{\left(\begin{array}{l}
U \tau \\
I
\end{array}\right)} \frac{\left(x-x_{g}\right)}{R(x)} S^{\prime}(x) f_{0}^{\prime}\left\{\frac{U\left(\tau-\frac{x}{U}\right)}{M R(x)}\right\} d x\right. \\
& \left.+\left(U \tau-x_{g}\right) S^{\prime}(U \tau)\right\}
\end{aligned}
$$

where

$$
\begin{aligned}
\left(U \tau-x_{\mathrm{g}}\right) S^{\prime}(U \tau) & =\left.\left(x-x_{g}\right) S^{\prime}(x)\right|_{X=U T} \text { for } 0 \leq U \tau \leq I \\
& =0 \text { for } U T>I
\end{aligned}
$$

and

where

$$
\begin{aligned}
& \operatorname{lin}_{m}(\tau)_{i}=q\left\{\frac{2}{M} \int_{0}^{\left(\begin{array}{l}
U T \\
I
\end{array}\right)} \frac{Y_{m}(x)}{R(x)} S^{\prime}(x) f_{0}^{1}\left\{\frac{U\left(\tau-\frac{x}{U}\right)}{M R(x)}\right\} d x\right. \\
& \left.+S^{\prime}(U \tau) Y_{m}(U \tau)\right\}
\end{aligned}
$$

and the subscript $i$ denotes that these entities are due to a unit impulse vind profile. Equations (II-6) through (II-8) and (II-10) through (II-I2) define the indicial and impulsive transient aerodynamic forces. These functions will be used in the following section to describe the forces for the Saturn C-5 configurations.

Before proceedinf, it should be pointed out that the impulsive forces can be obtained from the first time derivative of the indicial forces. Thus, 
in the following sections the indicial forces will be developed first. The latter forces will then be differential to obtain the desired expressions for the impulsive forces.

B. Development of Transient Indicial and Impulsive Aerodynamic Forces for The Saturn C-5 Configuration

The basic configuration of the Saturn C-5 missile is given in Fig. 41. The nose spike on the front of the missize corresponds to the escape tower which is attached to the vehicle throughout the boost flight. The geometry of the configuration is defined in Table $V$ with conic characteristics defined by $\beta_{n}=\tan \alpha_{n}$ for $n=0,1,2,3$.

\section{TABLE V}

\section{DEFINITION OF SATURN C-5 BODY GECMETRY}

Body Radius

$\mathrm{R}(\mathrm{x})$

$\beta_{\mathrm{O}} \mathrm{x}$

$R_{0}$

$\beta_{1}\left(x-x_{1}\right)+R_{0}$

$R_{I}$

$\beta_{2}\left(x-x_{3}\right)+R_{1}$

$\mathrm{R}_{2}$

$\beta_{3}\left(x-x_{5}\right)+R_{2}$

$\mathrm{R}_{3}$
Area Derivative

$$
\begin{gathered}
\frac{S^{\prime}(x)}{2 \pi \beta_{0}^{2} x} \\
0 \\
2 \pi \beta_{1}\left[\beta_{1}\left(x-x_{1}\right)+R_{0}\right] \\
0 \\
2 \pi \beta_{2}\left[\beta_{2}\left(x-x_{3}\right)+R_{1}\right]
\end{gathered}
$$

0

$$
2 \pi \beta_{3}\left[\beta_{3}\left(x-x_{5}\right)+R_{2}\right]
$$

0
Region

Applicable

$0 \leq x \leq x_{0}$

$x_{0} \leq x \leq x_{1}$

$x_{1} \leq x \leq x_{2}$

$x_{2} \leq x \leq x_{3}$

$x_{3} \leq x \leq x_{4}$

$x_{4} \leq x \leq x_{5}$

$x_{5} \leq x \leq x_{6}$

$x_{6} \leq x \leq I$

(II-6) yields

Substituting the appropriate definitions of the body geometry into 


$$
\mathbb{N}_{S}(\tau)=\mathbb{N}_{S}^{(1)}(\tau)+N_{S}^{(2)}(\tau)+N_{S}^{(3)}(\tau)+N_{S}^{(4)}(\tau)
$$

where the components $\mathbb{N}_{S}^{(1)}(\tau), \mathbb{N}_{S}^{(2)}(\tau), \mathbb{N}_{S}^{(3)}(\tau)$ and $\mathbb{N}_{S}^{(4)}(\tau)$ correspond to the normal force growth on the first, second, third and fourth missile regions, respectively (see Fig. 4I). These components are defined as follows: for the first region (escape tower)

$$
N_{S}^{(I)}(\tau)=\frac{2 q}{U} \int_{0}^{\left(\begin{array}{l}
U \tau \\
x_{0}
\end{array}\right)} 2 \pi \beta_{0}^{2} x f_{0}\left\{\frac{U\left(\tau-\frac{x}{U}\right)}{M F_{0} x}\right\} d x \quad 0 \leq \tau<\infty,
$$

for the second region (nose cone)

$$
\mathbb{N}_{S}^{(2)}(\tau)=\frac{2 q}{U} \int_{x_{1}}^{\left(\begin{array}{l}
U T \\
x_{2}
\end{array}\right)} 2 \pi B_{1}\left[\beta_{1}\left(x-x_{1}\right)+R_{0}\right] f_{\circ}\left\{\frac{U\left(\tau-\frac{x}{U}\right)}{M\left[\beta_{1}\left(x-x_{1}\right)+R_{0}\right]}\right\} d x
$$

for the third region

$$
N_{S}^{(3)}(\tau)=\frac{2 q}{U} \int_{x_{3}}^{\left(\begin{array}{l}
U T \\
x_{4}
\end{array}\right)} 2 \pi B_{2}\left[B_{2}\left(x-x_{3}\right)+R_{1}\right] f_{\circ}\left\{\frac{U\left(\tau-\frac{x}{U}\right)}{M\left[B_{2}\left(x-x_{3}\right)+R_{1}\right]}\right\} d x
$$


and for the fourth region (main booster)

$$
\mathbb{N}_{S}^{(4)}(\tau)=\frac{2 q}{U} \int_{x_{5}}^{\left(\begin{array}{l}
U \tau \\
x_{6}
\end{array}\right)} 2 \pi B_{3}\left[\theta_{3}\left(x-x_{5}\right)+R_{2}\right] f_{0}\left\{\frac{U\left(\tau-\frac{x}{U}\right)}{M\left[B_{3}\left(x-x_{5}\right)+R_{2}\right]}\right\} d x
$$

The limits of integration in the above expressions follow from a consideration of the various penetration times of the individual regions.

Carrying out the above integrations and simplifying for computation, the transient indicial normal force is given by

$$
N_{S}(\tau)=N_{S}^{(1)}(\tau)+\mathbb{N}_{S}^{(2)}(\tau)+N_{S}^{(3)}(\tau)+N_{S}^{(4)}(\tau)
$$

where the quantity $N_{S}^{(n)}(\tau)(n=1,2,3,4)$ depends on the value of the independent variable, $\tau$, and is defined by the following two expressions:

$$
\mathbb{N}_{s}^{(n)}(\tau)=\frac{2 q}{U}\left\{\begin{array}{c}
a_{n}\left(\tau-b_{n}\right)^{2}\left[I-2 f_{n} G\left(\frac{\tau-a_{n}}{e_{n}} ; f_{n}\right)\right]-c_{n} \\
\text { for } a_{n} \leq \tau \leq \bar{d}_{n} \\
h_{n}-2 f_{n} a_{n}\left(\tau-b_{n}\right)^{2}\left[G\left(\frac{\tau-d_{n}}{e_{n}} ; f_{n}\right)-G\left(\frac{\tau-\bar{a}_{n}}{\bar{e}_{n}} ; f_{n}\right)\right] \\
\text { for } \bar{a}_{n} \leq \tau<\infty, \\
n=1,2,3,4
\end{array}\right.
$$

The coefficients in (II-18) are defined in Table VI for the four regions of the $\mathrm{C}-5$. 
TABLE VI

DEFINITION OF COEFFICIENTS IN (II-18), (II-25), AND (II-36)

$\begin{array}{lllllllllllll}\underline{n} & \underline{a_{n}} & \underline{b_{n}} & \underline{c_{n}} & \underline{a_{n}} & \underline{\bar{d}_{n}} & \underline{e_{n}} & \underline{e_{n}} & \underline{f_{n}} & \underline{b_{n}} & \underline{x_{n}} & \underline{l_{n}} & \underline{R e g i o n} \\ 1 & \pi R_{0}^{2} U^{2} & 0 & 0 & 0 & \frac{x_{0}}{U} & 0 & \frac{M R_{0}}{U} & M B_{0} & S_{0} & \frac{2 S_{0} x_{0}}{3} & \frac{S_{0} x_{0}^{2}}{2} & \text { First }\end{array}$

$2 \pi B_{1}^{2} U^{2} \quad \frac{1}{U}\left(x_{1}-\frac{R_{0}}{\beta_{1}}\right) \quad S_{0} \quad \frac{x_{1}}{U} \quad \frac{x_{2}}{U} \quad \frac{M R_{0}}{U} \quad \frac{M R_{1}}{U} \quad M B_{1} \quad S_{1}-S_{0} \quad \frac{2\left(S_{1} R_{1}-S_{0} R_{0}\right)}{3 \beta_{1}} \quad \frac{S_{1} R_{1}^{2}-S_{0} R_{0}^{2}}{2 \beta_{1}^{2}} \quad$ Second

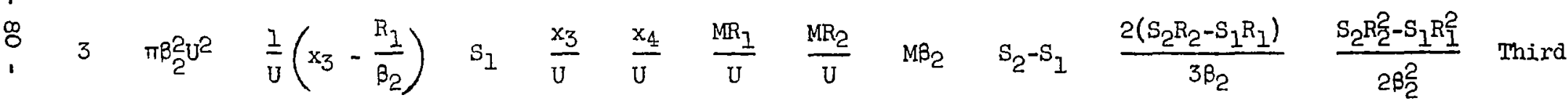

$4 \quad \pi \beta_{3}^{2} U^{2} \quad \frac{I}{U}\left(x_{5}-\frac{R_{2}}{\beta_{3}}\right) \quad S_{2} \quad \frac{x_{5}}{U} \quad \frac{x_{6}}{U} \quad \frac{M R_{2}}{U} \quad \frac{M R_{3}}{U} \quad M B_{3} \quad S_{3}-S_{2} \quad \frac{2\left(S_{3} R_{3}-S_{2} R_{2}\right)}{3 B_{3}} \quad \frac{S_{3} R_{3}^{2}-S_{2} R_{2}^{2}}{2 B_{3}^{2}}$ Fourth 
The function $G(\gamma ; 0)$ in $(I I-18)$ is defined as

$$
G(\gamma ; a)=\int_{0}^{\gamma} \frac{1-f_{0}(y)}{(1+a y)^{3}} d y
$$

where $f_{0}(y)$ is given by (II-4) and $\alpha$ corresponds to the product $M B_{n}$ (see Table II). $\beta_{n}$ is the tangent of a particular region connection angle, $a_{n}$.

Next, we consider the moment growth on the C-5. Substituting the appropriate definitions of the body geometry into (II-7) yields

$$
M_{S}(\tau)=M_{S}^{(1)}(\tau)+M_{S}^{(2)}(\tau)+M_{S}^{(3)}(\tau)+M_{S}^{(4)}(\tau)
$$

where

$$
\begin{array}{r}
M_{S}^{(1)}(\tau)=x_{g N} N_{S}^{(1)}(\tau)-\frac{2 q}{U} \int_{0}^{\left(\begin{array}{l}
U \tau \\
x_{0}
\end{array}\right)} x \cdot 2 \pi R_{O}^{2} f_{0}\left\{\frac{U\left(\tau-\frac{x}{U}\right)}{M B_{0} x}\right\} d x \\
0 \leq \tau<\infty,
\end{array}
$$

$M_{S}^{(2)}(\tau)=x_{G} N_{S}^{(2)}(\tau)-\frac{2 q}{U} \int_{x_{I}}^{\left(\begin{array}{l}U T \\ x_{2}\end{array}\right)} x \cdot 2 \pi \beta_{I}\left[\beta_{I}\left(x-x_{I}\right)+R_{0}\right] f_{0}\left\{\frac{U\left(\tau-\frac{x}{U}\right)}{M\left[\beta_{I}\left(x-x_{I}\right)+R_{0}\right]}\right\} d x$
$\frac{x_{I}}{U} \leq \tau<\infty$,

$$
\begin{aligned}
& M_{S}^{(3)}(\tau)=x_{g^{3 N}} N_{S}^{(3)}(\tau)-\frac{2 q}{U} \int_{x_{3}}^{\left(\begin{array}{c}
U \tau \\
x_{4}
\end{array}\right)} x \cdot 2 \pi B_{2}\left[\beta_{2}\left(x-x_{3}\right)+R_{1}\right] f_{\circ}\left\{\frac{U\left(\tau-\frac{x}{U}\right)}{M\left[\beta_{2}\left(x-x_{3}\right)+R_{1}\right]}\right\} d x \\
& \frac{X_{3}}{U} \leq \tau<\infty,
\end{aligned}
$$


and

$$
\begin{aligned}
& M_{S}^{(4)}(\tau)=x_{g} N_{S}^{(4)}(\tau)-\frac{2 q}{U} \int_{x_{5}}^{\left(\begin{array}{c}
U \tau \\
x_{6}
\end{array}\right)} x \cdot 2 \pi \beta_{3}\left[\beta_{3}\left(x-x_{5}\right)+R_{2}\right] f_{\circ}\left\{\frac{U\left(\tau-\frac{x}{U}\right)}{M\left[\beta_{3}\left(x-x_{5}\right)+R_{2}\right]}\right\} d x \\
& \frac{\mathrm{X}_{5}}{\mathrm{U}} \leq \tau<\infty \text {, }
\end{aligned}
$$

Now, carrying out the above integrations and simplifying, the transient indicial moment is given by

$$
M_{S}(\tau)=M_{S}^{(1)}(\tau)+M_{S}^{(2)}(\tau)+M_{S}^{(3)}(\tau)+M_{S}^{(4)}(\tau)
$$

where the quantity $M_{S}^{(n)}(\tau)(n=1,2,3,4)$ depends on the independent variable, $\tau$, and is defined by the following two expressions:

$$
\begin{aligned}
M_{S}^{(n)}(\tau)= & \left(x_{g}-U b_{n}\right) N_{s}^{(n)}(\tau) \\
& -\frac{2 q}{U}\left\{\begin{array}{c}
\frac{2 a_{n} U}{3}\left(\tau-b_{n}\right)^{3}\left[1-3 f_{n} H\left(\frac{\tau-d_{n}}{e_{n}} ; f_{n}\right)\right]-\frac{2 U c_{n} e_{n}}{3 f_{n}} \\
a_{n} \leq \tau \leq \bar{a}_{n} \\
k_{n}-2 f_{n} a_{n} U\left(\tau-b_{n}\right)^{3}\left[H\left(\frac{\tau-a_{n}}{e_{n}} ; f_{n}\right)-H\left(\frac{\tau-\bar{d}_{n}}{\bar{e}_{n}} ; f_{n}\right)\right] \\
\bar{a}_{n} \leq \tau<\infty, \\
n=1,2,3,4 .
\end{array}\right.
\end{aligned}
$$

The function $H(\gamma ; a)$ in (II-25) is defined as 


$$
H(\gamma ; \alpha)=\int_{0}^{\gamma} \frac{1-f_{0}(y)}{\left(1+\alpha_{y}\right)^{4}} d y
$$

Now, we will consider the derivation of the transient indicial forces corresponding to the bending coordinates. From (II-8) it is seen that a description of the mode shapes, $Y_{m}(x)$, is needed in order to evaluate the $Q_{m}(\tau)_{s}{ }^{\prime} s$. The mode shape data for the Saturn C-5 were available only in discrete numerical form. These data vere inconvenient to use in this form since they prevented evaluation of (II-8) in a manner similar to that used for (II-6) and (II-7). However, by approximating the mode shapes with polymomials, the preparation of (II-8) for computation could be readily accomplished.

From (II-8) it is easily seen that the evaluation of the integral. requires information about the mode shape only over those sections of the missile which have a changing radius. Thus the mode shape approximations need only apply over relatively short streamuise distances. From an analysis of the first four bending modes of the C-5 configuration, it was found that quadratic polynomials could be used efficiently to approximate $Y_{m}(x)$ with an acceptable degree of accuracy. In this instance, the quadratic polynomial mode shapes yielded values which were within 1 per cent of the actual mode deflections.

Now, let the mode shapes $(m=1,2,3,4)$ in the desired region (see Fig. 41 ) be approximated by

$$
\begin{aligned}
& Y_{m}(x)=\bar{A}_{I m}+\bar{B}_{1 m} x+\bar{C}_{1 m} x^{2} \text { in First Region } \\
& Y_{m}(x)=\bar{A}_{2 m}+\bar{B}_{2 m} x+\bar{C}_{2 m} x^{2} \text { in Second Region } \\
& Y_{m}(x)=\bar{A}_{3 m}+\bar{B}_{3 m} x+\bar{C}_{3 m} x^{2} \text { in Third Region }
\end{aligned}
$$

and

$$
Y_{m}(x)=\bar{A}_{4 m}+\bar{B}_{4 m} x+\bar{C}_{4 m} x^{2} \text { in Fourth Region }
$$


where the $\overline{\mathrm{A}}$ 's,$\overline{\mathrm{B}}^{\prime} \mathrm{s}$, and $\overline{\mathrm{C}}^{\prime} \mathrm{s}^{*}$ are considered constant for a discrete flight time or altitude.

Substituting (II-27) through (II-30) and the appropriate definitions of the body geometry into (II-8) yields

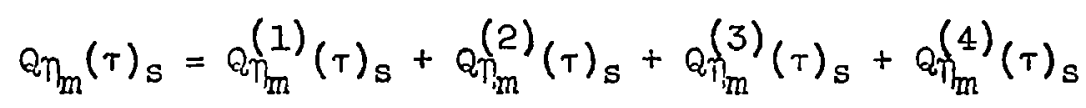

where

$$
\begin{aligned}
& Q_{\eta_{m}}^{(1)}(\tau)_{S}=\left(\bar{A}_{I m}+\bar{B}_{I m} x_{g}\right) N_{S}^{(I)}(\tau)-\bar{B}_{I m} M_{S}^{(1)}(\tau) \\
& +\bar{C}_{I m} \frac{2 \mathrm{q}}{\mathrm{U}} \int_{0}^{\left(\begin{array}{c}
U \tau \\
x_{0}
\end{array}\right)} \mathrm{x}^{2} \cdot 2 \pi \beta_{0}^{2} x_{0}\left\{\frac{U\left(\tau-\frac{x}{U}\right)}{M_{O^{x}}}\right\} d x \\
& 0 \leq \tau<\infty \text {, } \\
& q_{m}^{(2)}(\tau)_{S}=\left(\bar{A}_{2 m}+\bar{B}_{2 m} x_{G}\right) N_{S}^{(2)}(\tau)-\bar{B}_{2 m} M_{s}^{(2)}(\tau) \\
& +\bar{c}_{2 m} \frac{2 q}{U} \int_{x_{1}}^{\left(\begin{array}{c}
U \tau \\
x_{2}
\end{array}\right)} x^{2} \cdot 2 \pi \beta_{1}\left[B_{1}\left(x-x_{1}\right)+R_{0}\right] f_{0}\left\{\frac{U\left(\tau-\frac{x}{U}\right)}{M\left[\beta_{1}\left(x-x_{1}\right)+R_{0}\right]}\right\} d x \\
& \frac{x_{1}}{U} \leq \tau<\infty,
\end{aligned}
$$

* The $\bar{A}$ 's , $\bar{B}$ 's and $\bar{C}$ 's for the first four bending modes were computed between flight times of 10 and $140 \mathrm{sec}$. at 10-sec. intervals. However, values for only the first two bending modes between 30 and $100 \mathrm{sec}$., inclusive, were used in the numerical computation of the indicial and impulsive responses. 


$$
\begin{aligned}
& Q \eta_{m}^{(3)}(\tau)_{S}=\left(\bar{A}_{3 m}+\bar{B}_{3 m} x_{G}\right) N_{S}^{(3)}(\tau)-\bar{B}_{3 m^{M}} M_{S}^{(3)}(\tau) \\
& +\bar{c}_{3 m} \frac{2 q}{U} \int_{x_{3}}^{\left(\begin{array}{c}
U \tau \\
x_{4}
\end{array}\right)} x^{2} \cdot 2 \pi \beta_{2}\left[\beta_{2}\left(x-x_{3}\right)+R_{1}\right] f_{0}\left\{\frac{U\left(\tau-\frac{x}{U}\right)}{M\left[\beta_{2}\left(x-x_{3}\right)+R_{1}\right]}\right\} d x \\
& \frac{X_{3}}{U} \leq \tau<\infty,
\end{aligned}
$$

and

$$
\begin{aligned}
& Q \eta_{m}^{(4)}(\tau)_{S}=\left(\bar{A}_{4 m}+\bar{B}_{4 m_{B}} x_{g}\right) N_{S}^{(4)}(\tau)-\bar{B}_{4 m} M_{S}^{(4)}(\tau) \\
& +\bar{C}_{4 m} \frac{2 q}{U} \int_{x_{5}}^{\left(\begin{array}{l}
U \tau \\
x_{6}
\end{array}\right)} x^{2} \cdot 2 \pi \beta_{3}\left[\beta_{3}\left(x-x_{5}\right)+R_{2}\right] f_{\circ}\left\{\frac{U\left(\tau-\frac{x}{U}\right)}{M\left[\beta_{3}\left(x-x_{5}\right)+R_{2}\right]}\right] d x \\
& \frac{\mathrm{X}_{5}}{\mathrm{U}} \leq \tau<\infty \text {. }
\end{aligned}
$$

Performing the above integrations and simplifying, the transient indicial bending moments are given by

$$
Q_{\eta_{m}}(\tau)_{s}=Q_{\eta_{m}}^{(1)}(\tau)_{s}+Q_{\eta_{m}}^{(2)}(\tau)_{s}+Q_{\eta}(3)(\tau)_{s}+Q_{\eta_{m}}^{(4)}(\tau)_{s}
$$

where 


$$
\begin{aligned}
Q_{\eta_{m}}^{(n)}(\tau)_{s} & =\left\{\bar{A}_{n m}-\bar{c}_{n m}\left(U b_{n}\right)^{2}+x_{g}\left[\bar{B}_{n m}+2 \bar{c}_{n m} U b_{n}\right]\right\} N_{s}^{(n)}(\tau) \\
& -\left[\bar{B}_{n m}+2 \bar{C}_{n m} U b_{n}\right] M_{s}^{(n)}(\tau) \\
& +\frac{2 q}{U} \bar{c}_{n m}\left\{\begin{array}{l}
\frac{a_{n} U^{2}}{2}\left(\tau-b_{n}\right)^{4}\left[I-4 f_{n} I\left(\frac{\tau-a_{n}}{e_{n}} ; f_{n}\right)\right]-\frac{c_{n}}{2}\left(\frac{U e_{n}}{f_{n}}\right)^{2} \\
a_{n} \leq \tau \leq \bar{a}_{n} \\
l_{n}-2 f_{n} a_{n} U^{2}\left(\tau-b_{n}\right)^{4}\left[I \left(\frac{\tau-a_{n}}{\left.e_{n} ; f_{n}\right)}-I\left(\frac{\tau-\bar{d}_{n}}{\bar{e}_{n}} ; f_{n}\right)\right.\right.
\end{array}\right]
\end{aligned}
$$

The function $I(\gamma ; a)$ in $(I I-36)$ is defined as

$$
I(\gamma ; \alpha)=\int_{0}^{\gamma} \frac{1-f_{0}(y)}{\left(1+\alpha_{y}\right)^{5}} d y
$$

The advantages of using polynomial curve fits (especially quadratic polynomials) for the mode shapes, $Y_{m}(x)$, can be easily seen from (II-36). The evaluation of the $\theta \eta_{m}^{(n)}(\tau)_{s}$ 's can be made from the normal force, moment and an additional integral, I $(\gamma ; \alpha)$. If quadratic polynomials had not provided sufficient accuracy in describing the $Y_{m}{ }^{\prime} s$, the number of integrals to evaluate would be equal to one less than the order of polynomial approximation. However, for the C-5 configuration the transient indicial normal force, moment and bending moments can be expressed in terms of integrais similar to (II-19), (II-26), and (II-37). 
As discussed at the first of this Appendix, the normal force, moment and bending moments due to a unit impulse wind profile are given by the first time derivative of the indicial force and moments. Therefore, differentiating $(I I-13),(I I-20)$ and $(I I-3 I)$ with respect to time yields

$$
\begin{aligned}
& N_{i}(\tau)=\frac{d}{d \tau} N_{S}(\tau)=N_{i}^{(1)}(\tau)+N_{i}^{(2)}(\tau)+N_{i}^{(3)}(\tau)+N_{i}^{(4)}(\tau), \\
& M_{i}(\tau)=\frac{d}{d \tau} M_{S}(\tau)=M_{i}^{(I)}(\tau)+M_{i}^{(2)}(\tau)+M_{i}^{(3)}(\tau)+M_{i}^{(4)}(\tau)
\end{aligned}
$$

and

$$
Q_{\eta_{m}}(\tau)_{i}=\frac{d}{d \tau} Q_{\eta_{m}}(\tau)_{s}=Q_{\eta_{m}}^{(1)}(\tau)_{i}+Q_{\eta_{m}}^{(2)}(\tau)_{i}+Q_{\eta_{m}}^{(3)}(\tau)_{i}+Q_{\eta_{m}}^{(4)}(\tau)_{i}
$$

where

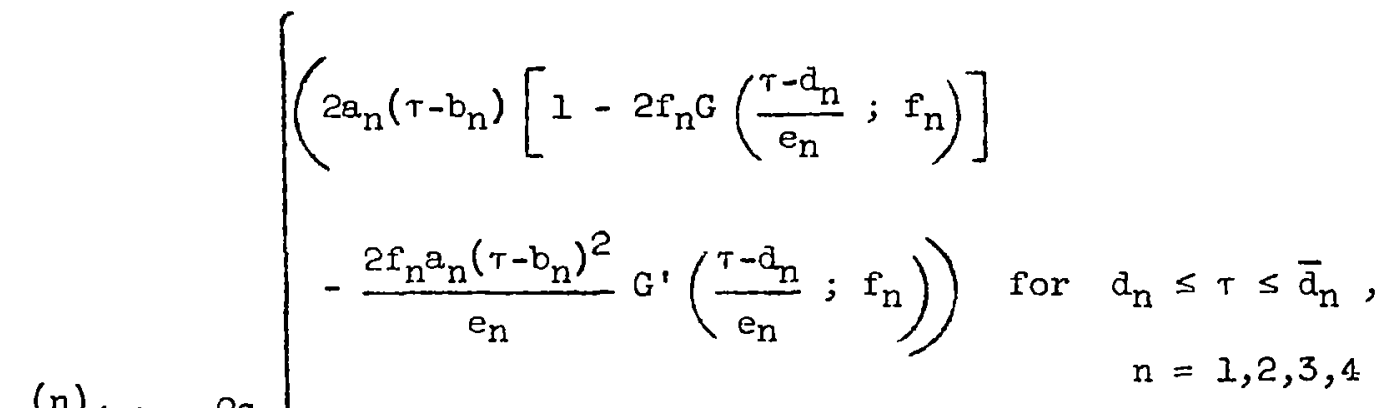

$$
\begin{aligned}
& N_{i}^{(n)}(\tau)=\frac{2 q}{U} \\
& \left(-4 f_{n} a_{n}\left(\tau-b_{n}\right)\left[G\left(\frac{\tau-d_{n}}{e_{n}} ; f_{n}\right)-G\left(\frac{\tau-\bar{d}_{n}}{\bar{e}_{n}} ; f_{n}\right)\right]\right. \\
& \left.-2 f_{n^{a}}\left(\tau-b_{n}\right)^{2}\left[\frac{1}{e_{n}} G^{\prime}\left(\frac{\tau-a_{n}}{e_{n}} ; f_{n}\right)-\frac{1}{\bar{e}_{n}} G^{\prime}\left(\frac{\tau-\bar{a}_{n}}{\bar{e}_{n}} ; f_{n}\right)\right]\right) \\
& \text { for } \overline{\mathrm{d}}_{\mathrm{n}} \leq \mathrm{T}<\infty \text {, } \\
& \mathrm{n}=1,2,3,4,
\end{aligned}
$$




$$
\begin{aligned}
& M_{i}^{(n)}(\tau)=\left(x_{g}-U b_{n}\right) N_{i}^{(n)}(\tau)
\end{aligned}
$$

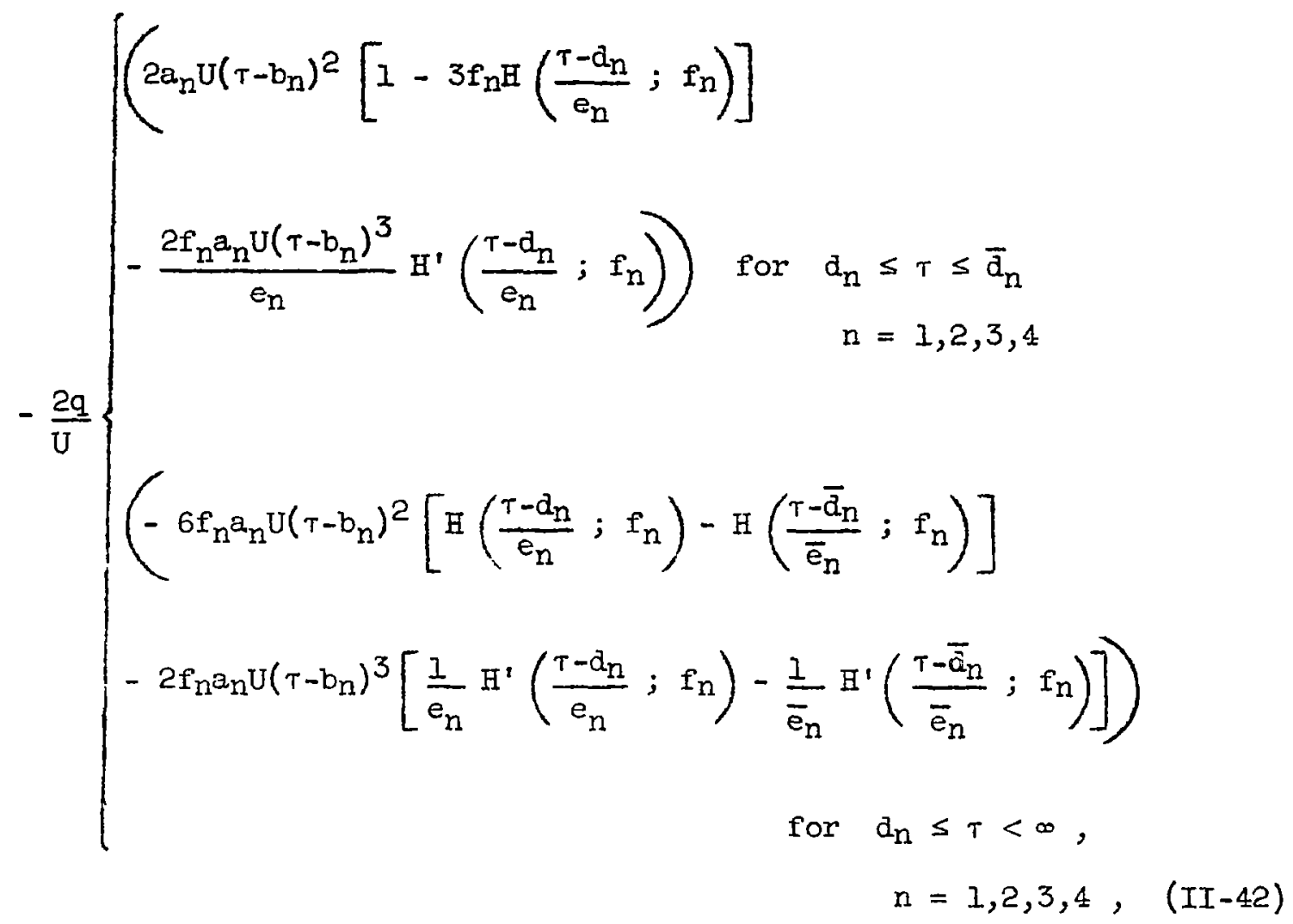

and 


$$
\begin{aligned}
& \mathrm{Q}_{\mathrm{m}}^{(\mathrm{n})}(\tau)_{\mathcal{I}}=\left\{\bar{A}_{n m}-\bar{C}_{n m}\left(U b_{n}\right)^{2}+x_{G}\left[\bar{B}_{n m}+2 \bar{C}_{n m} U_{b_{n}}\right]\right\} N_{i}^{(n)}(\tau) \\
& -\left[\bar{B}_{n m}+2 \bar{C}_{n m} \mathrm{Ub}_{n}\right] M_{i}^{(n)}(\tau)
\end{aligned}
$$

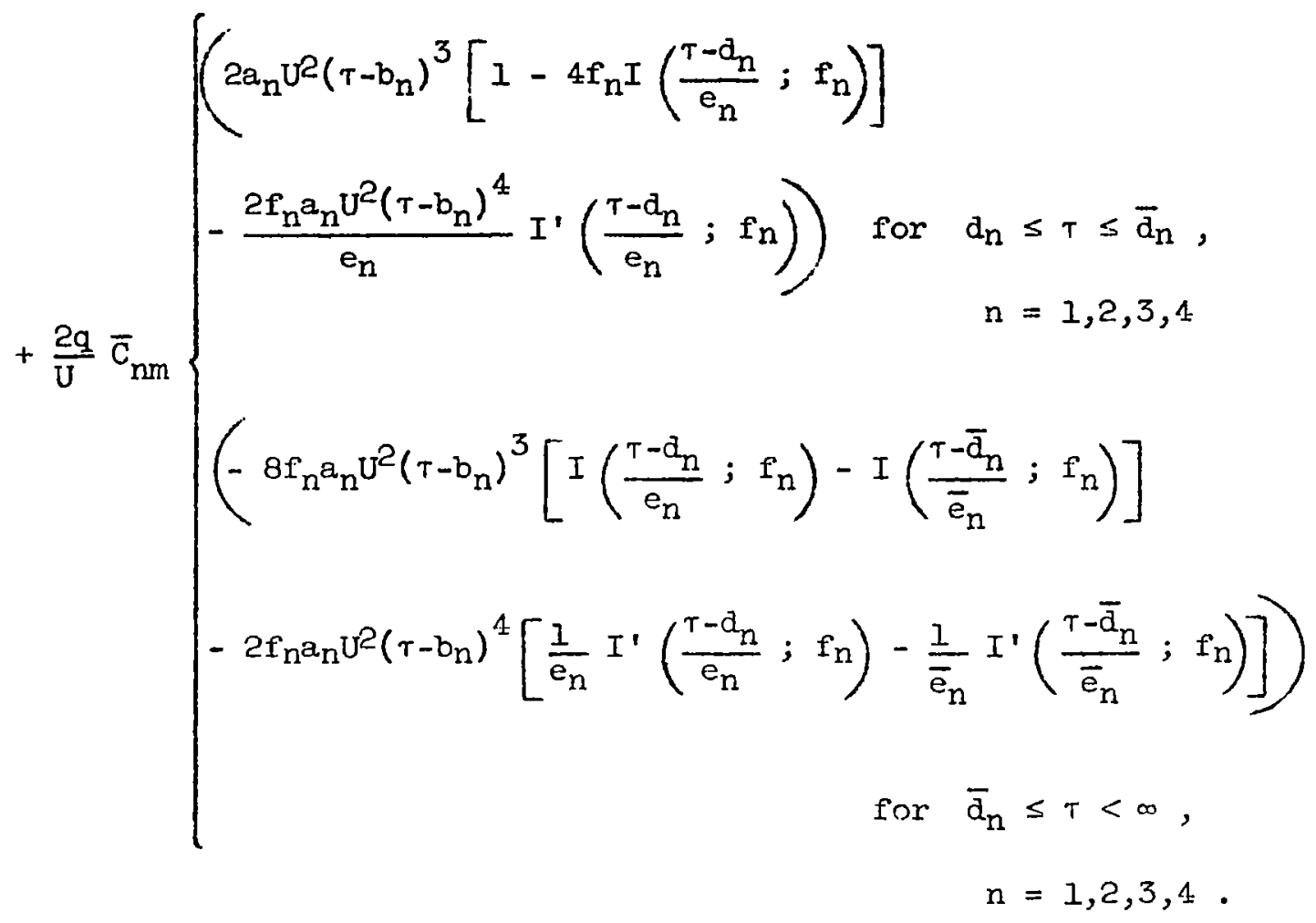

The functions $G^{\prime}(\gamma ; \alpha), H^{\prime}(\gamma ; \alpha)$ and $I^{\prime}(\gamma ; \alpha)$ are the respective derivatives of $\mathrm{G}(\gamma ; \alpha), \mathrm{H}(\gamma ; \alpha)$ and $\mathrm{I}(\gamma ; \alpha)$ :

$$
\begin{aligned}
& G^{\prime}(\gamma ; \alpha)=\frac{1-f_{O}(\gamma)}{(1+\alpha \gamma)^{3}} \\
& H^{\prime}(\gamma ; \alpha)=\frac{1-f_{O}(\gamma)}{(1+\alpha \gamma)^{4}}
\end{aligned}
$$

and 


$$
I^{\prime}(\gamma ; \alpha)=\frac{1-f_{0}(\gamma)}{(1+\alpha y)^{5}} .
$$

When $n=1$, the terms $\frac{1}{e_{n}} G^{\prime}\left(\frac{T-a_{n}}{e_{n}} ; f_{n}\right), \frac{1}{e_{n}} H^{\prime}\left(\frac{\tau-a_{n}}{e_{n}} ; f_{n}\right)$ and $\frac{I}{e_{n}} I\left(\frac{\tau-d_{n}}{e_{n}} ; f_{n}\right)$ in (II-4I), (II-42) and (II-43), respectively, must be omitted.

The equations (II-13), (II-18), (II-20), (II-25), (II-3I), (II-36), (II-38), (II-39) and (II-40) are the fundamental expressions required to compute the indicial and irmpulsive normal force, moment and bending morient growth functions for the Saturn C-5 configuration. These aerodynamic forces and moments were used in calculating the indicial and impulsive responses of the C -5 missile system for the case "penetration with lift growth."

C. Development of Quasi-Steady Indicial and Impulsive Aerodynamic Forces for the Saturn C-5 Configuration

The pure-penetration forcing functions are presented in this section. These functions are based on penetration geometrics and quasi-steady aerodynamics.

In penetration geometrics each station along the missile experiences the wind crossflow associated with the station altitude.

In the quasi-steady aerodynamics the crossflow velocity is assumed to be everywhere much smaller than the local speed of sound. Thus, the problem is one of solving Iaplace's equation in each crossflow plane. This approach is justified only in the case of low frequency oscillation of the vehicle at moderate Mach numbers.

We will consider first the penetration normal force, moment and bending moment growth functions due to a unit step wind profile. Assuming that the local speed of sound is essentially infinite with respect to the crossflow velocity, then

$$
\begin{gathered}
f_{O}\left\{\frac{U\left(\tau-\frac{x}{U}\right)}{M R}\right\}=I\left(\tau-\frac{x}{U}\right) . \\
-90-
\end{gathered}
$$


Substituting (II-47) into (II-6), (II-7) and (II-8) gives

$$
\mathbb{N}_{N}(\tau)=\frac{2 q}{U} \int_{0}^{\left(\begin{array}{l}
U T \\
L
\end{array}\right)} S^{\prime}(x) I\left(\tau-\frac{x}{U}\right) d x,
$$

and

$$
\operatorname{ar}_{m}(\tau)_{S}=\frac{2 q}{U} \int_{0}^{\left(U^{T}\right)} Y_{m}(x) S^{\prime}(x) I\left(\tau-\frac{x}{U}\right) d x .
$$

Substituting the appropriate definitions of the body geometry into (II-48), (II-49) and (II-50), recalling (II-27) through (II-30) and integrating the results gives: for the normal force due to penetration only,

$$
\begin{aligned}
& N_{S}(\tau)=N_{S}^{(1)}(\tau)+N_{S}^{(2)}(\tau)+N_{S}^{(3)}(\tau)+N_{S}^{(4)}(\tau) \\
& \mathrm{MR} \rightarrow 0 \quad \mathrm{MR} \rightarrow 0 \quad \mathrm{MR} \rightarrow 0 \quad \mathrm{MR} \rightarrow 0 \quad \mathrm{MR} \rightarrow 0
\end{aligned}
$$

where

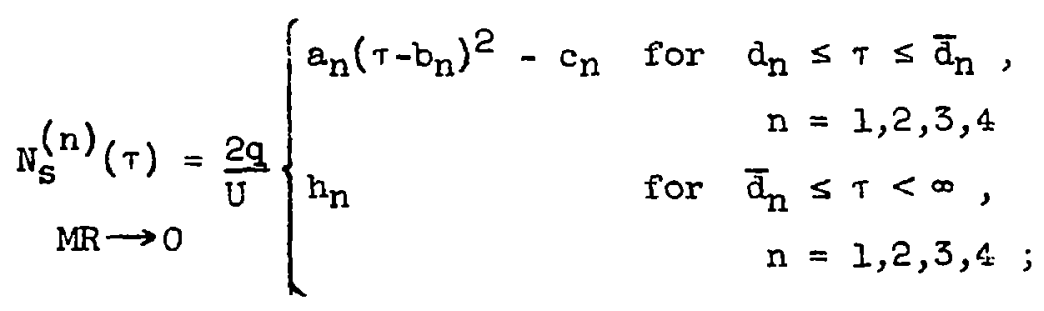


for the moment due to penetration only,

$$
\begin{aligned}
& M_{S}(\tau)=M_{S}^{(1)}(\tau)+M_{S}^{(2)}(\tau)+M_{S}^{(3)}(\tau)+M_{S}^{(4)}(\tau) \\
& \underset{M R \rightarrow 0}{M R} \rightarrow 0 \quad M R \rightarrow 0 \\
& M R \rightarrow 0 \quad M R \rightarrow 0
\end{aligned}
$$

where

$$
\begin{aligned}
& M_{S}^{(n)}(\tau)=\left(x_{g}-U b_{n}\right) N_{S}^{(n)}(\tau) \\
& \mathrm{MR} \rightarrow 0 \quad \mathrm{MR} \rightarrow 0 \\
& -\frac{2 q}{U}\left\{\begin{array}{lrl}
\frac{2 a_{n} U}{3}\left(\tau-b_{n}\right)^{3}-\frac{2 U c_{n} e_{n}}{3 f_{n}} & \text { for } \quad a_{n} \leq \tau \leq \bar{a}_{n} \\
n & =1,2,3,4 \\
k_{n} & \text { for } \bar{d}_{n} \leq \tau<\infty, \\
& n=1,2,3,4 ;
\end{array}\right.
\end{aligned}
$$

and for the bending moment due to penetration only,

$$
\underset{\mathrm{MR} \rightarrow 0}{Q_{\eta_{\mathrm{m}}}(\tau)_{\mathrm{S}}}=\underset{\mathrm{MR} \rightarrow 0}{Q_{\eta_{\mathrm{m}}}^{(1)}(\tau)_{\mathrm{S}}}+\underset{\mathrm{MR} \rightarrow 0}{Q \eta_{\mathrm{m}}^{(2)}(\tau)_{\mathrm{S}}}+\underset{\mathrm{MR} \rightarrow 0}{Q_{\eta_{\mathrm{m}}}^{(3)}(\tau)_{\mathrm{s}}}+\underset{\mathrm{MR} \rightarrow 0}{Q_{\mathrm{m}}^{(4)}(\tau)_{\mathrm{S}}}
$$

where 


$$
\begin{aligned}
& \underset{\mathrm{MR} \rightarrow 0}{\mathrm{Q} \eta_{m}^{(n)}(\tau)_{s}}=\left\{\bar{A}_{n m}-\bar{C}_{n m}\left(\mathrm{Ub}_{n}\right)^{2}+x_{\mathrm{g}}\left[\bar{B}_{n m}+2 \bar{C}_{n m} \mathrm{ub}_{n}\right]\right\} \underset{\mathrm{MR} \rightarrow 0}{N_{s}^{(n)}(\tau)} \\
& -\left[\bar{B}_{n m}+2 \bar{C}_{n m} U_{n}\right] \underset{M R \rightarrow 0}{M_{s}^{(n)}(\tau)}
\end{aligned}
$$

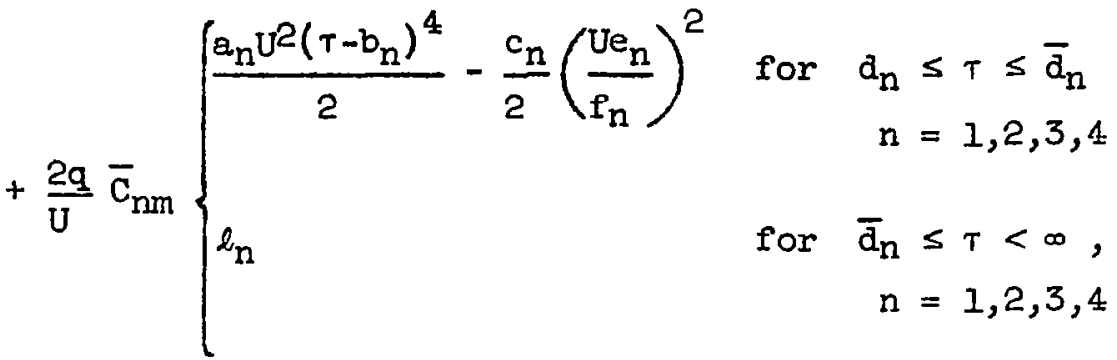

The penetration normal force, moment and bending moment due to a unit impulse wind profile are obtained from (II-52), (II-53) and (II-55), respectively, upon differentiating with respect to time. Thus, for the normal force

$$
\begin{aligned}
& N_{i}(\tau)=N_{i}^{(1)}(\tau)+N_{i}^{(2)}(\tau)+N_{i}^{(3)}(\tau)+N_{i}^{(4)}(\tau) \\
& \mathrm{MR} \rightarrow 0 \quad \mathrm{MR} \rightarrow 0 \quad \mathrm{MR} \rightarrow 0 \quad \mathrm{MR} \rightarrow 0 \quad \mathrm{MR} \rightarrow 0
\end{aligned}
$$

where

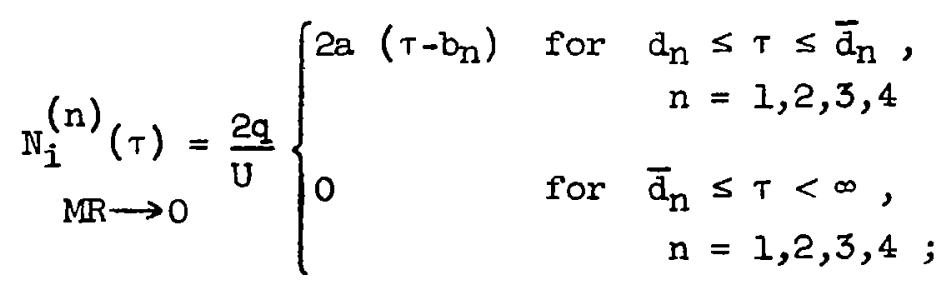

for the moment,

$$
\begin{aligned}
& M_{i}(\tau)=M_{i}^{(1)}(\tau)+M_{i}^{(2)}(\tau)+M_{i}^{(3)}(\tau)+M_{i}^{(4)}(\tau) \\
& \mathrm{MR} \rightarrow 0 \quad \mathrm{MR} \rightarrow 0 \quad \mathrm{MR} \rightarrow 0 \quad \mathrm{MR} \rightarrow 0 \quad \mathrm{MR} \rightarrow 0
\end{aligned}
$$


where

$$
\underset{M R \rightarrow 0}{M_{i}^{(n)}(\tau)=\left(x_{g}-U b_{n}\right) N_{i}^{(n)}(\tau)} \underset{M R \rightarrow 0}{\rightarrow \rightarrow 0}
$$

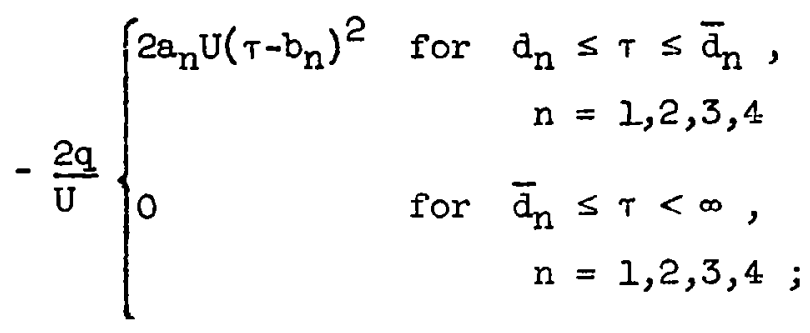

and for the bending moments,

$$
\begin{aligned}
& Q_{\eta_{m}}(\tau)_{i}=Q \eta_{m}^{(1)}(\tau)_{i}+Q \eta_{m}^{(2)}(\tau)_{i}+Q \eta_{m}^{(3)}(\tau)_{i}+Q \eta_{m}^{(4)}(\tau)_{i} \\
& \mathrm{MR} \rightarrow 0 \quad \mathrm{MR} \rightarrow 0 \quad \mathrm{MR} \rightarrow 0 \quad \mathrm{MR} \rightarrow 0 \quad \mathrm{MR} \rightarrow 0
\end{aligned}
$$

where

$$
\begin{aligned}
& Q_{m}^{(n)}(\tau)_{i}=\left\{\bar{A}_{n m}-\bar{C}_{n m}\left(U b_{n}\right)^{2}+x_{g}\left[\bar{B}_{n m}+2 \bar{C}_{n m} U b_{n}\right]\right\} \underset{M R \rightarrow 0}{N_{i}^{(n)}(\tau)} \\
& -\left[\bar{B}_{n m}+2 \bar{C}_{n m} \mathrm{Ub}_{n}\right] M_{i}^{(n)}(\tau) \\
& \mathrm{MR} \rightarrow 0
\end{aligned}
$$

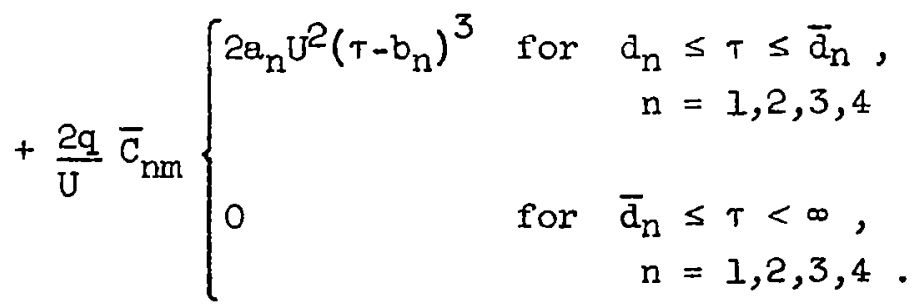


The equations (II-5I), (II-53), (II-55), (II-57), (II-59) and (II-6I) are the fundamental expressions required to compute the penetration indicial and impulsive force and moments for the $\mathrm{C}-5$ configuration. The coefficients given in these equations are defined in Table II. These forces and moments were used in calculating the indicial and impulsive responses of the $\mathrm{C}-5$ missile system for the case of "pure penetration."

\section{Development of the Steady Indicial and Impulsive Aerodynamic Forces for the Saturn C-5 Configuration}

This section presents the instantaneous-immersion forcing functions. These functions are based on instantaneous-immersion geometrics and quasisteady aerodynamics. In this geometric consideration every station along the missile experiences the same wind-induced crossflow, namely that crossflow occurring at the nose.

We will consider first the steady normal force, moment and bendinc moments due to a unit step wind profile. Referring to (II-6), (II-7), and (II-8), the indicial forces resulting from instantaneous immersion are

$$
\begin{gathered}
\bar{N}_{S}(\tau)=\frac{2 q}{U} I(\tau) \int_{0}^{L} S^{\prime}(x) d x \\
\bar{M}_{S}(\tau)=-\frac{2 q}{U} I(\tau) \int_{0}^{L}\left(x-x_{G}\right) S^{\prime}(x) d x
\end{gathered}
$$

and

$$
\bar{Q}_{\eta_{m}}(\tau)_{S}=\frac{2 q}{U} I(\tau) \int_{0}^{L} Y_{m}(x) S^{\prime}(x) d x
$$

It should be noted that the aerodynamic coefficients in the above expressions are identical to the coefficients multiplying the translational velocity terms in the rigid body and bending equations of motion (see Appendix I 
$(I-1),(I-2)$ and $(I-3))$. Thus, (II-63), (II-64) and (II-65) can be rewritten* as

$$
\begin{aligned}
& \bar{N}_{S}(\tau)=\frac{2 Q F_{O}}{U} I(\tau), \\
& \bar{M}_{S}(\tau)=-\frac{2 Q F_{I}}{U} I(\tau)
\end{aligned}
$$

and

$$
Q_{T_{m}}(\tau)_{s}=\frac{2 Q D_{m}}{U} I(\tau)
$$

respectively, where $I(\tau)$ is the Heaviside step function.

The instantaneous-immersion normal force, moment and bending moments due to a unit impulse wind profile are obtained from (II-66), (II-67) and (II-68) upon differentiating with respect to time:

$$
\begin{aligned}
& \overline{\mathbb{N}}_{1}(\tau)=\frac{2 Q F_{O}}{U} \delta(\tau) \\
& \bar{M}_{1}(\tau)=-\frac{2 Q F_{1}}{U} S(\tau)
\end{aligned}
$$

and

$$
\bar{Q}_{\eta_{m}}(\tau)_{i}=\frac{2 Q D_{m}}{U} \delta(\tau)
$$

* The evaluation of $\overline{\mathbb{N}}_{S}(\tau), \bar{M}_{S}(\tau)$ and $\bar{Q}_{r_{m}}(\tau)_{S}$ can also be obtained from (II-52), (II-54) and (II-56) by setting $\tau$ equal to infinity. 
where $\delta(\tau)$ is the Dirac delta function.

For $\tau>0, \bar{N}_{i}(\tau), \bar{M}_{i}(\tau)$ and $\bar{Q}_{\eta_{m}}(\tau)_{i}$ are identically equal to zero (see (II-58), (II-60) and (II-62) for $\tau=\infty$ ).

The equations (II-66) through (II-71) are the fundamental expressions required to compute the instantaneous-immersion indicial and impulsive force and moments. These forces were used in calculating the indicial and impulsive responses of the Saturn C-5 missile system for the case of "Instantaneous immersion." 


\section{CALCULATION OF INITTAL AND STEADY-STATE CONDITTONS RESULTING} FROM A UNIT IMPUISE AND UNIT STEP WIND PROFILE

The numerical solution for the indicial and impulstve responses of the vehicle requires a knowledge of the initial conditions imposed by the varlous forcing functions (see Appendix II). Since the Runge Kutta method of solution is used, the initial conditions for the generalized coordinates and their first derivatives are needed.

The steady-state values for the indicial and impulsive responses of the vehicle are required for computer logic which terminates the integration when response is sufficiently close to its steady value (see Vol. III).

A procedure is given in this Appendix for computing the initial and steady-state conditions for the impulsive and indicial responses of the vehicle. For simplicity, the method is described for a system defined by the following generalized coordinates (see Appendix I): translation, rotation, first and second bending, first and second sloshing and control deflection. The procedure is general in nature, however, and can be applied to a system with more degrees of freedom.

The initial and steady-state conditions are obtained through usage of Laplace transform techniques. The initial and steady-state conditions are found in the limit of the transform as the Laplace variable approaches infinity and zero, respectively. The above conditions are derived for six cases of forcing functions: unit impulse and step considering penetration with lift growth, pure penetration and instantaneous-immersion effects.

\section{CALCUIATION OF INITIAL CONDITIONS}

Writing the equations of motion $[$ see (I-II) through (I-19)] as a set of first order equations, we find

$$
[A]\{\dot{q}\}+[B]\{q\}=\{C\}
$$


where the elements $a_{i j}$ and $b_{i j}(i, j=1,2, \ldots, 18)$ of the square matrices [A] and [B], respectively, correspond to the coefficients on the left hand side of (I-II) through (I-19),

$$
\{q\}=\left\{\begin{array}{c}
\mathrm{y}_{0} \\
\phi \\
\eta_{1} \\
\eta_{2} \\
\xi_{1} \\
\bar{s}_{2} \\
\beta_{\mathrm{C}} \\
\mathrm{N} \\
\mathrm{p} \\
\dot{\mathrm{y}}_{0} \\
\dot{\phi} \\
\dot{\eta}_{1} \\
\dot{\eta}_{2} \\
\dot{\xi}_{1} \\
\dot{\xi}_{2} \\
\dot{\beta}_{\mathrm{C}} \\
\dot{\mathrm{N}} \\
\dot{\mathrm{p}}
\end{array}\right\}
$$

and $\{c\}$ is a column matrix of forcing functions. Six different sets of forcing functions were considered in the numerical investigation presented in this report.

For conveniences of comparison the elements, $c_{i},(i=1,2, \ldots, 18)$ of $\{c\}$ are given in Table VII for a unit impulse wind profile $\left\{v_{y}(t)=\delta(t)\right\}$ and $a$ unit step wind profile $\left\{v_{y}(t)=I(t)\right\}$ considering penetration with lift growth (PWLG), pure penetration (PP) and instantaneous-immersion (II) effects.

Expressions for the aerodynamic quantities in Table VII are given in Appendix II. 
ELEMENTSS OF FORCING FUNCTION MATRIX $\{c\}$

\begin{tabular}{|c|c|c|c|c|c|c|}
\hline \multirow[b]{2}{*}{$\underline{i}$} & \multicolumn{6}{|c|}{$c_{i}$} \\
\hline & FWLG & PP & II & FWLG & $\underline{P P}$ & II \\
\hline$I$ & 0 & 0 & 0 & 0 & 0 & 0 \\
\hline 2 & 0 & 0 & 0 & 0 & 0 & 0 \\
\hline 3 & 0 & 0 & 0 & 0 & 0 & 0 \\
\hline 4 & 0 & 0 & 0 & 0 & 0 & 0 \\
\hline 5 & 0 & 0 & 0 & 0 & 0 & 0 \\
\hline 6 & 0 & 0 & 0 & 0 & 0 & 0 \\
\hline 7 & 0 & 0 & 0 & 0 & 0 & 0 \\
\hline 8 & 0 & 0 & 0 & 0 & 0 & 0 \\
\hline 9 & 0 & 0 & 0 & 0 & 0 & 0 \\
\hline 10 & $N_{i}(\tau)$ & $\begin{array}{l}N_{i}(T) \\
\quad M R \rightarrow 0\end{array}$ & $\bar{N}_{i}(\tau)$ & $\mathbb{N}_{s}(\tau)$ & $\begin{array}{l}N_{s}(\tau) \\
M R \rightarrow 0\end{array}$ & $\overline{\mathbb{N}}_{\mathrm{S}}(\tau)$ \\
\hline 11 & $M_{i}(\tau)$ & $\begin{array}{l}M_{i}(\tau) \\
M R \rightarrow 0\end{array}$ & $\bar{M}_{1}(\tau)$ & $M_{S}(\tau)$ & $\begin{array}{l}M_{S}(\tau) \\
M R \rightarrow 0\end{array}$ & $\bar{M}_{S}(\tau)$ \\
\hline 12 & $Q_{\eta}(\tau)_{i}$ & $\underbrace{Q_{M}}_{I}(\tau)_{i}$ & $\bar{Q}_{r_{1}}(\tau)_{i}$ & $Q_{\eta_{I}}(\tau)_{S}$ & $\underbrace{Q_{\eta_{1}}(\tau)_{S}}_{M R}$ & $\bar{Q}_{\eta_{1}}(\tau)$ \\
\hline 13 & $Q_{\eta_{2}}(T)_{i}$ & $\begin{array}{l}{ }_{\eta_{2}}(\tau)_{i} \\
M R \rightarrow 0\end{array}$ & $\bar{Q}_{\eta_{2}}(\tau)_{i}$ & ${ }^{Q} \eta_{2}(\tau)_{s}$ & ${ }_{\substack{\eta_{2} \\
M}}(\tau)_{S}$ & $\bar{Q}_{\eta}(\tau)$ \\
\hline 14 & 0 & 0 & 0 & 0 & 0 & 0 \\
\hline 15 & 0 & 0 & 0 & 0 & 0 & 0 \\
\hline 16 & 0 & 0 & 0 & 0 & 0 & 0 \\
\hline 17 & 0 & 0 & 0 & 0 & 0 & 0 \\
\hline 18 & $\frac{b_{0} \delta(\tau)}{U}$ & $\frac{b_{0} \delta(\tau)}{U}$ & $\frac{b_{0} \delta(\tau)}{U}$ & $\frac{b_{0} I(\tau)}{U}$ & $\frac{b_{0} I(\tau)}{U}$ & $\frac{b_{0} I(\tau)}{U}$ \\
\hline
\end{tabular}


Now, the initial conditions, $\{\mathrm{q}(0)\}$, can be found readily by using Iaplace transform techniques. Taking the Laplace transform of (III-I) yields

$$
[\overline{\mathrm{AB}}]\{\overline{\mathrm{q}}\}=\{\overline{\mathrm{C}}\}
$$

where

$$
\{\bar{q}\}=\mathcal{L}\{q\}
$$

and

$$
[\overline{\mathrm{AB}}]=\mathrm{s}[\mathrm{A}]+[\mathrm{B}]
$$

matrix $\{c\}$ are given in Table VIII for the six different sets of forcing functions. The symbol $Q\left(\frac{1}{s}\right)$ denotes "the order of $\frac{1}{s}$."

The initial conditions, ${ }^{*}\{q(0)\}$, are now obtained by solving (III-3) for each dependent variable by Cramer's rule, multiplying the ratio of two determinants by $s$ and taking the limit as $s \longrightarrow \infty$.

The initial conditions imposed by a unit impulse wind are given in Table IX for the cases where penetration with lift growth and pure penetration effects are considered.

* If $\bar{f}(s)$ is given as the Laplace transform of $f(t)$, then the initial condition, $f\left({ }^{+} \circ\right)$, 1s found from

$$
\lim _{s \rightarrow \infty} s \bar{f}(s)=f\left(+_{0}\right)
$$

where $s$ is the Laplace transform variable. 
TABLE VIII

ELEMENTS OF THE TRANS FORMED FORCING FUNCTION MATRIX $\{\overline{\mathrm{C}}\}$

$\bar{c}_{i}$

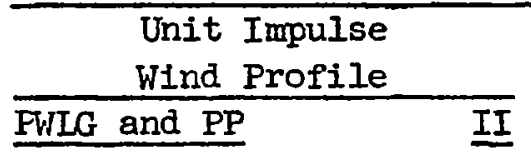

$0 \quad 0$

0

0

0

0

0

0

0

0

0

0

$O\left(\frac{1}{s}\right)$

$\frac{2 Q F_{O}}{U}$

11

$\vartheta\left(\frac{1}{5}\right)$

$\frac{-2 Q F_{1}}{U}$

12

13

$O\left(\frac{1}{s}\right)$

$\frac{2 Q D_{1}}{U}$

$O\left(\frac{1}{s}\right)$

\section{II}

0

0

o

0

0

0

$$
\frac{2 Q D_{2}}{U}
$$

14

15

16

17

18
0

0

0

0

$\frac{b_{0}}{U}$
0

0

0

0

$\frac{b_{0}}{U}$
Unit step

Wind Profile

PWIG and PP

$\underline{I I}$

0

0

0

0

0

0

0

0

0

0

0

0

0

0

0

0

$C^{\top j}\left(\frac{1}{s}\right)$

$C\left(\frac{1}{s}\right)$

$G\left(\frac{1}{5}\right)$

$O\left(\frac{1}{s}\right)$

0

0

0

0

$\frac{b_{0}}{U} \frac{1}{s}$
0

0

$\frac{2 Q F_{O}}{U} \frac{1}{s}$

$\frac{-2 Q F_{1}}{U} \frac{1}{s}$

$\frac{2 Q D_{1}}{U} \frac{1}{s}$

$\frac{2 \mathrm{QD}_{2}}{\mathrm{U}} \frac{1}{\mathrm{~s}}$
0

0

0

0

$\frac{\mathrm{b}_{\mathrm{O}}}{\mathrm{U}} \frac{1}{\mathrm{~s}}$ 
INITIAL CONDITIONS IMPOSED BY A UNIT IMPULSE WIND

FOR THE CASES OF PENETRATION VITH LIFT GROWTH

AND PURE PENETRATION

$\begin{array}{ll}\mathrm{y}_{0}(0)=0 & \dot{\mathrm{y}}_{0}(0)=0 \\ \phi(0)=0 & \dot{\phi}(0)=0 \\ \eta_{1}(0)=0 & \dot{\eta}_{1}(0)=0 \\ \eta_{2}(0)=0 & \dot{\eta}_{2}(0)=0 \\ \xi_{1}(0)=0 & \dot{\xi}_{1}(0)=0 \\ \xi_{2}(0)=0 & \dot{\xi}_{2}(0)=0 \\ \beta_{C}(0)=0 & \dot{f}_{C}(0)=0 \\ N(0)=0 & \dot{N}(0)=0 \\ P(0)=0 & \dot{P}(0)=b_{0} \\ & \end{array}$

The initial conditions imposed by a unit impulse wind for the case of instantaneous immersion are:

$$
\begin{array}{ll}
\mathrm{y}_{0}(0)=0 & \mathrm{~N}(0)=0 \\
\phi(0)=0 & \mathrm{P}(0)=0 \\
\eta_{1}(0)=0 & \dot{R}_{\mathrm{C}}(0)=0 \\
\eta_{2}(0)=0 & \dot{\mathrm{N}}(0)=0 \\
\xi_{1}(0)=0 & \\
\xi_{2}(0)=0 & \\
\beta_{C}(0)=0 &
\end{array}
$$


In addition, solutions for $\dot{y}_{0}(0), \dot{\phi}(0), \dot{\eta}_{1}(0), \dot{\eta}_{2}(0), \dot{\xi}_{1}(0)$ and $\dot{\xi}_{2}(0)$ are obtained from the matrix equation

$$
\{z(0)\}=[D]^{-1}\{J\}
$$

where

$$
\{z(0)\}=\left\{\begin{array}{l}
\dot{\mathrm{j}}_{0}(0) \\
\dot{\sigma}_{(}(0) \\
\dot{\eta}_{I}(0) \\
\dot{\eta}_{2}(0) \\
\dot{\xi}_{1}(0) \\
\dot{\xi}_{2}(0)
\end{array}\right\} \text { and }\{J\}=\left\{\begin{array}{c}
\frac{2 Q F_{0}}{U} \\
\frac{-2 Q F_{1}}{U} \\
\frac{2 Q D_{1}}{U} \\
\frac{2 Q D_{2}}{\sigma} \\
0 \\
0
\end{array}\right\}
$$

The elements, $d_{i j}$, of the square matrix $[D]$ correspond to the inertia coefficients in (I-II) through (I-16) (see Appendix I).

Finally, the initial conditions imposed by a unit step wind are given in Table $X$ for the cases where penetration with lift growth, pure penetration and instantaneous-imersion effects are considered. 


\section{TABLE $\mathrm{X}$}

INITIAL CONDITIONS IMPOSED BY A UNIT STEP WIND FOR

THE CASES OF PENETRATION WITH LIFT GROWTH, PURE

PENETRATION AND INSTANTANEOUS IMMERS ION

$$
\begin{array}{ll}
\mathrm{y}_{0}(0)=0 & \dot{\mathrm{y}}_{0}(0)=0 \\
\phi(0)=0 & \dot{\phi}(0)=0 \\
\eta_{1}(0)=0 & \dot{\eta}_{1}(0)=0 \\
\eta_{2}(0)=0 & \dot{\eta}_{2}(0)=0 \\
\xi_{1}(0)=0 & \dot{\xi}_{1}(0)=0 \\
\xi_{2}(0)=0 & \dot{\xi}_{2}(0)=0 \\
B_{C}(0)=0 & \dot{\xi}_{C}(0)=0 \\
N(0)=0 & \dot{N}_{N}(0)=0 \\
P(0)=0 & \dot{\mathrm{P}}(0)=0
\end{array}
$$

II. CALCUIATION OF STEADY-STATE VALUES

The procedure for computing the steady-state values of the impulsive and Indicial responses follows the analysis given in section $I$ except for the following changes:

1. The first order system of equations of motion [see (III-1)] are written in terms of $\dot{y}_{0}$ and $\dot{y}_{0}$ instead of $y_{0}$ and $\dot{y}_{0}$ to avoid an indeterminant form.

2. The steady state values of the impulsive responses are the same for the three aerodynamic environments considered.* The same is true of the steady state values of the indicial responses. Thus, the steady state values for the impulsive and indicial responses will be found from the case of instantaneous immersion. 
3. The steady state conditions are obtained when the Laplace transform variable goes to zero in the limit.**

Omitting the details, the steady state values of the impulsive and indicial responses are given in Tables VII and VIII, respectively.

TABLE XI

\section{STEADY STATE VALUES FOR}

IMPULSIVE RESPONSES

TABLE XII

STEADY STATE VALUES FOR

INDICIAL RESPONSES
$\dot{y}_{0}(\infty)=0$
$\ddot{\mathrm{y}}_{0}(\infty)=0$
$\dot{y}_{0}(\infty)=1$
$\ddot{\mathrm{y}}_{0}(\infty)=0$
$\phi(\infty)=0$
$\dot{\phi}(\infty)=0$
$\phi(\infty)=0$
$\dot{\phi}(\infty)=0$
$\eta_{I}(\infty)=0$
$\dot{\eta}_{1}(\infty)=0$
$\eta_{1}(\infty)=0$
$\dot{\eta}_{1}(\infty)=0$
$n_{2}(\infty)=0$
$\dot{\eta}_{2}(\infty)=0$
$n_{2}(\infty)=0$
$\dot{\eta}_{2}(\infty)=0$
$\xi_{1}(\infty)=0$
$\dot{\xi}_{1}(\infty)=0$
$\xi_{I}(\infty)=0$
$\dot{\xi}_{l}(\infty)=0$
$\xi_{2}(\infty)=0$
$\dot{\xi}_{2}(\infty)=0$
$\xi_{2}(\infty)=0$
$\dot{\xi}_{2}(\infty)=0$
$\beta_{C}(\infty)=0$
$\dot{B}_{C}(\infty)=0$
$\beta_{C}(\infty)=0$
$\dot{\beta}_{C}(\infty)=0$
$N(\infty)=0$
$\dot{N}(\infty)=0$
$N(\infty)=0$
$\dot{N}(\infty)=0$
$P(\infty)=0$
$\dot{P}(\infty)=0$
$P(\infty)=0$
$\dot{\mathrm{P}}(\infty)=0$

* The steady state values of impulsive forcing functions, given in Table III, are the same for the three aerodynamic environments. The same is true for the steady state values of the indicial forcing functions. Since the forcing function vector is the only quantity which changes in the set of equations [see (III-1)] for the different aerodymamic environments, it is easily seen that the above statement is correct.

* If $\bar{f}(s)$ is the Laplace transform of $f(t)$, then the steady state value, $f\left(+_{\infty}\right)$, is found from

$$
\lim _{s \rightarrow 0} \bar{f}(s)=f\left(+_{\infty}\right)
$$




\section{APPENDIX IV \\ PRESENTATION OF EXTREME EXCURSION AND \\ AVERAGE RESPONSE PLOTS}

This Appendix contains the extreme excursion and local average response plots discussed in Section $V$. The method of analysis used to obtain these plots is discussed in Section IV. 


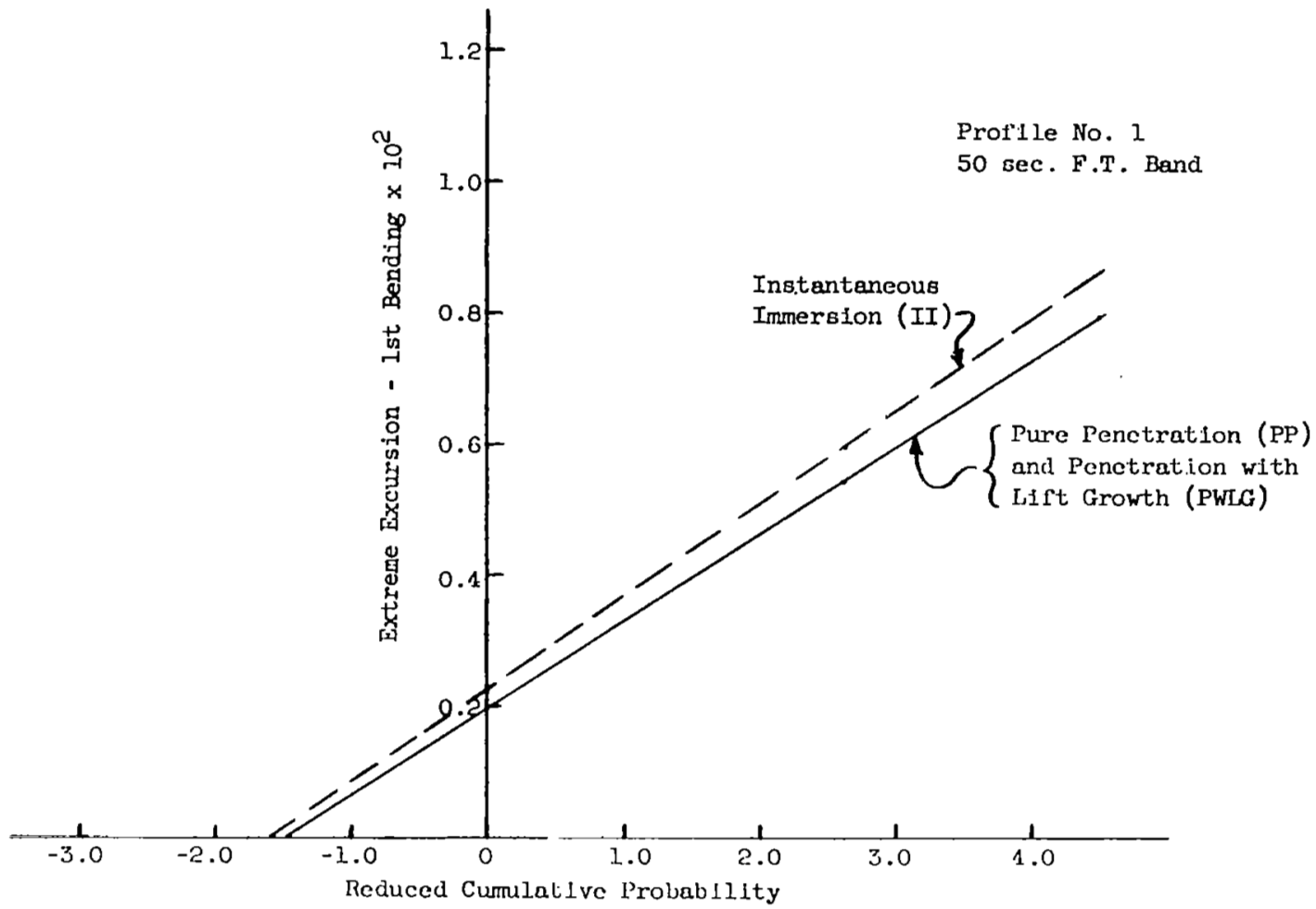

F1C. 42 - Extreme Excursion of lst Bending vs. Reduced Cumulative Probability - $50 \mathrm{sec}$. F.T.

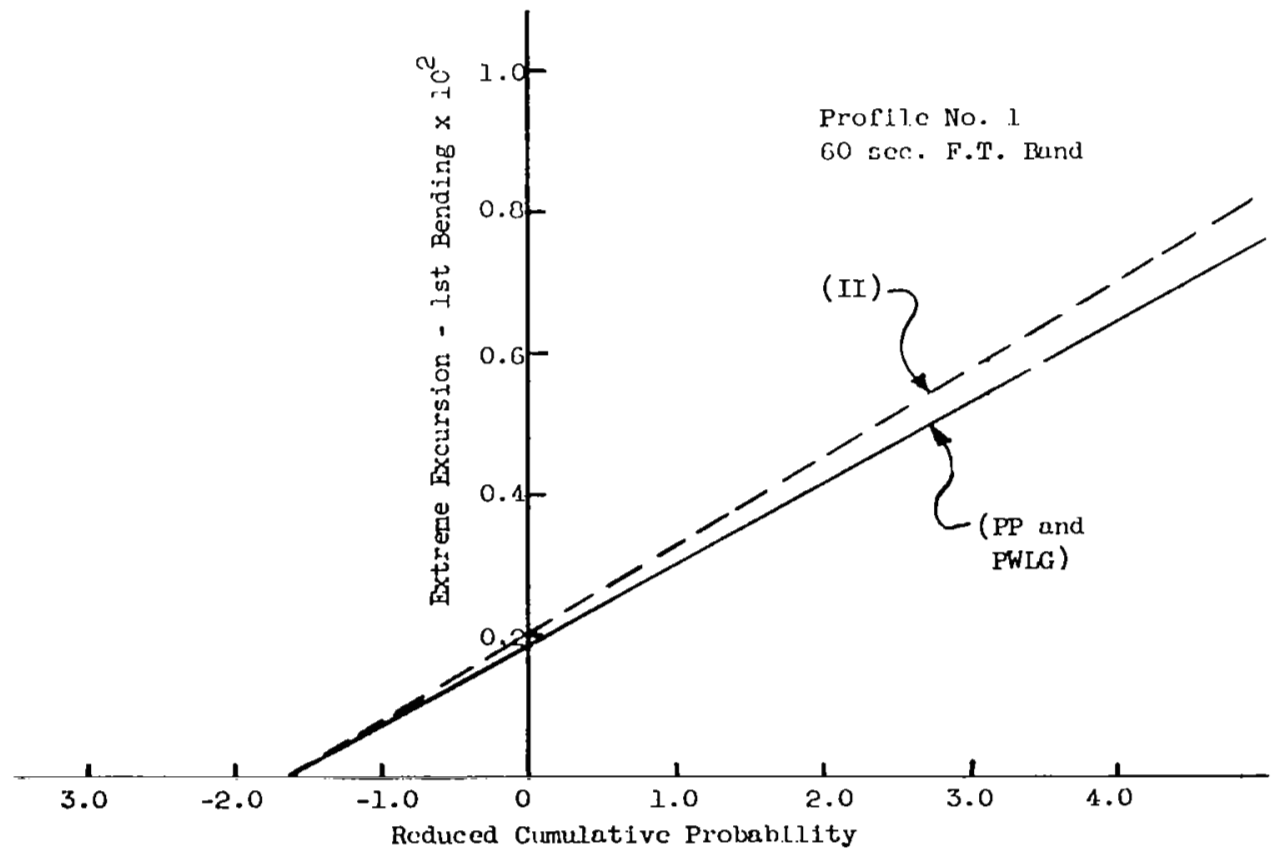

Fig. 43 - Extreme Excursion of lst Bending vs. Reduced Cumulative Probability - $60 \mathrm{sec}$. F.T. 


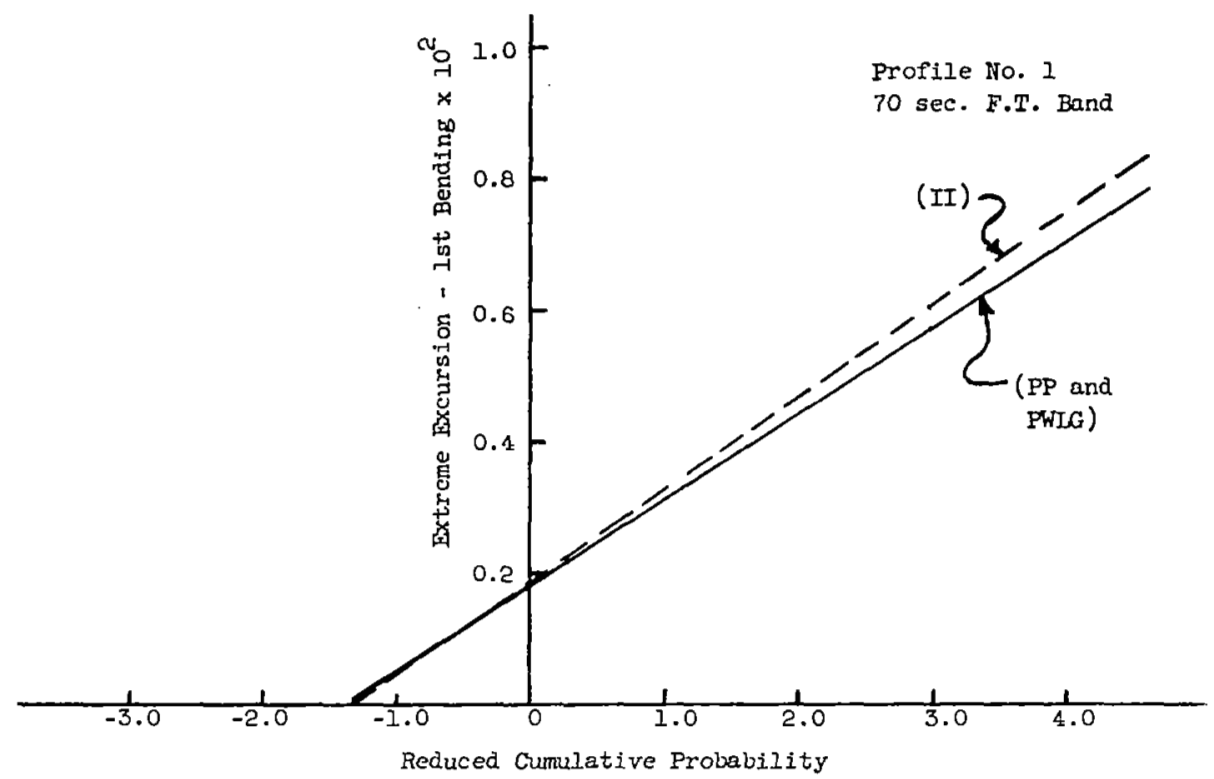

Fig. 44 - Extreme Excursion of lst Bending vs. Reduced Cumulative Probability - $70 \mathrm{sec}$. F.T.

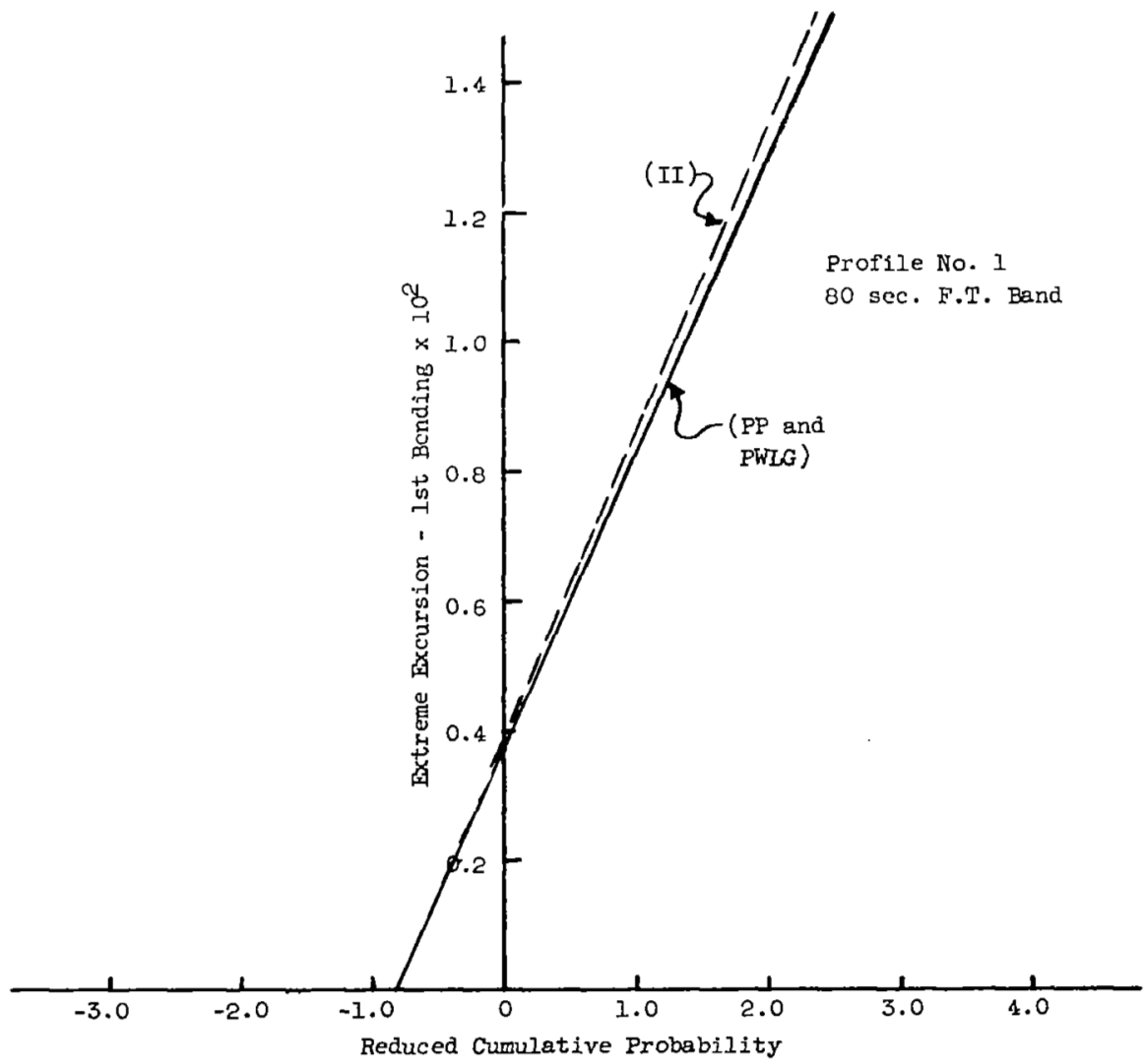

Fic. 15 - Extreme Excursion of lst Bending vs. Reduced Cumulative Probability - $80 \mathrm{sec}$. F.T. 


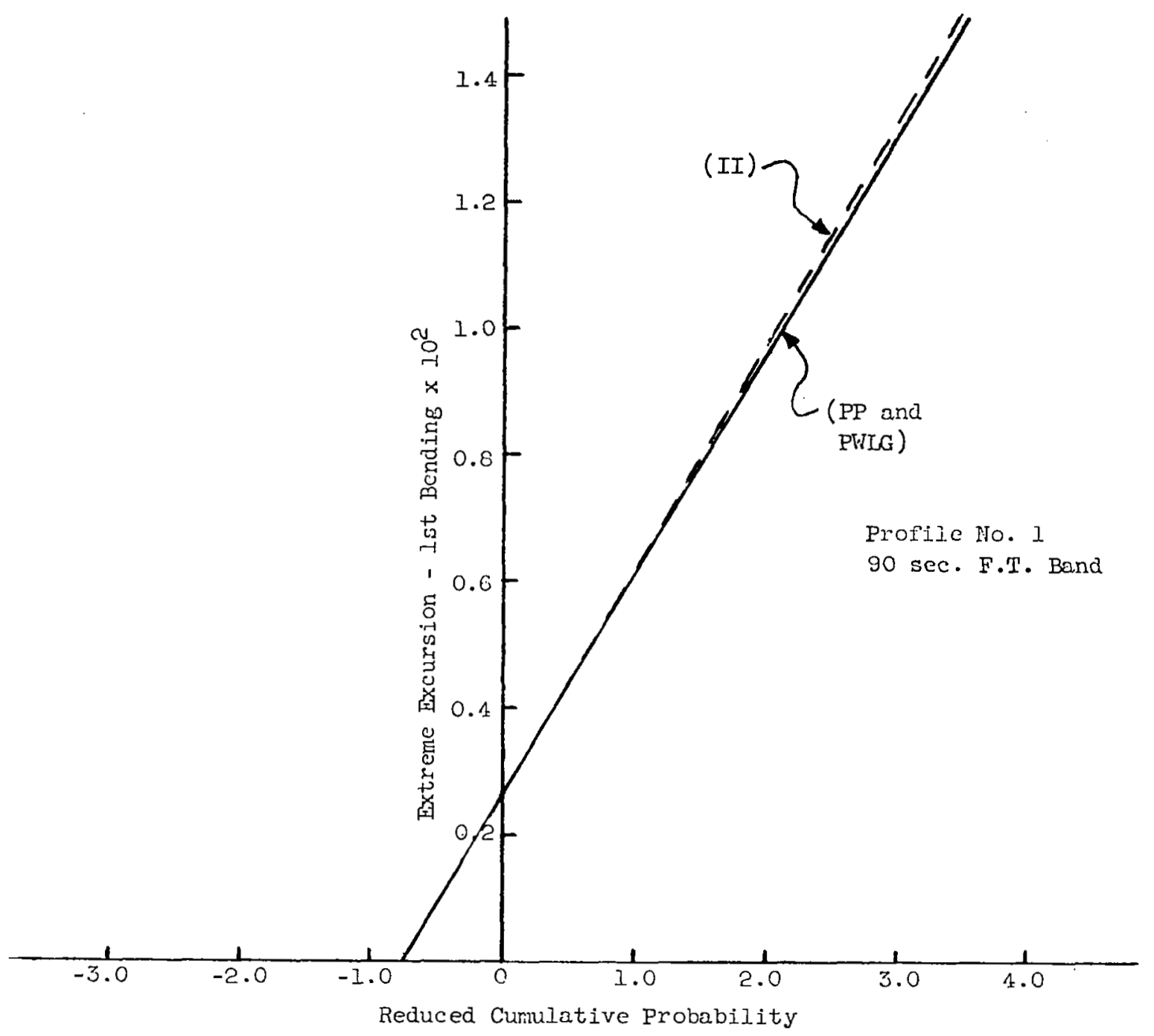

Fig. 46 - Extreme Excursion of lst Bending vs. Reduced Cumulative Probability - $90 \mathrm{sec}$. F.T. 


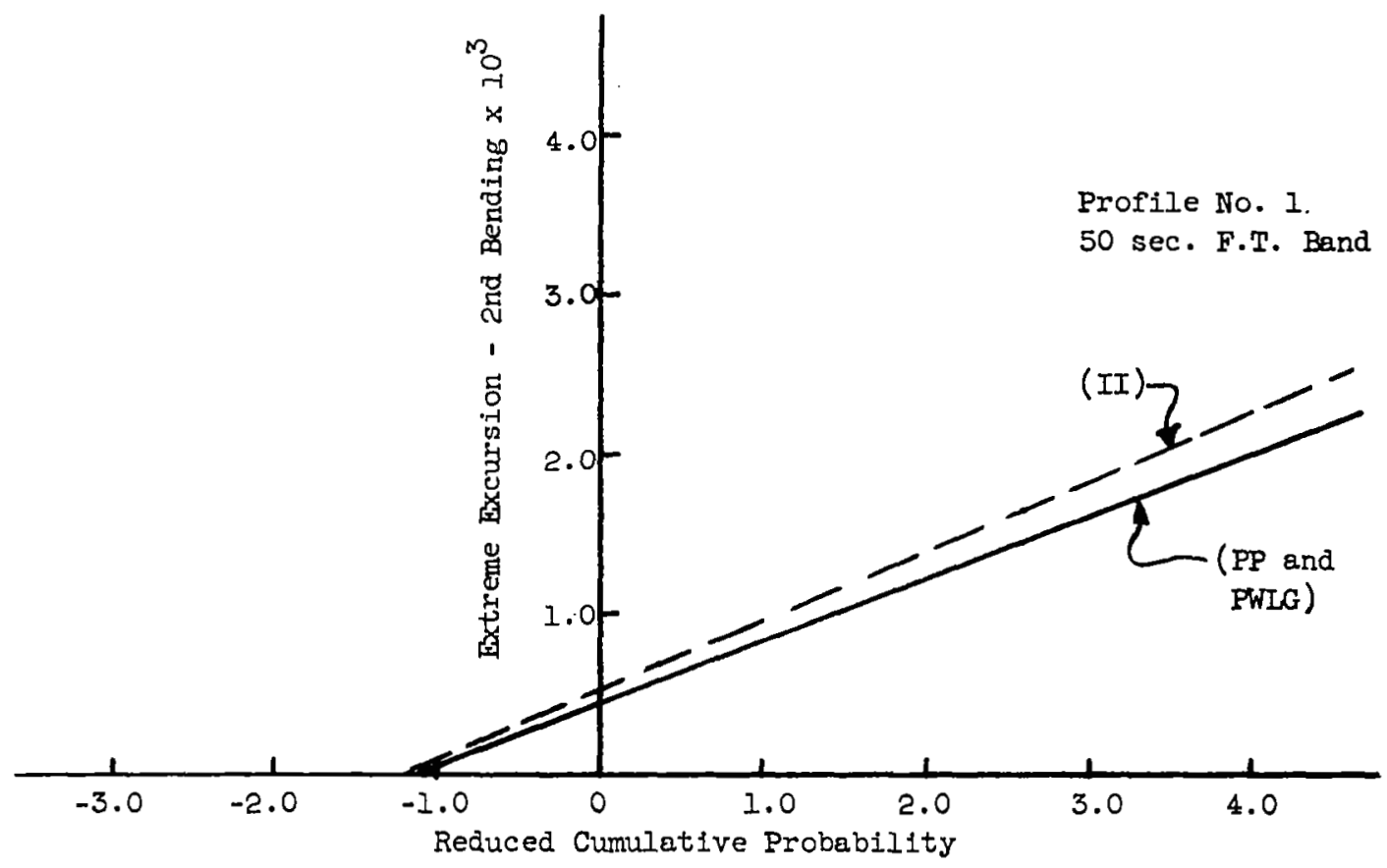

Fic. 47 - Extreme Excursion of 2nd Bending vs. Reduced Cumulative Probability - 50 sec. F.T.

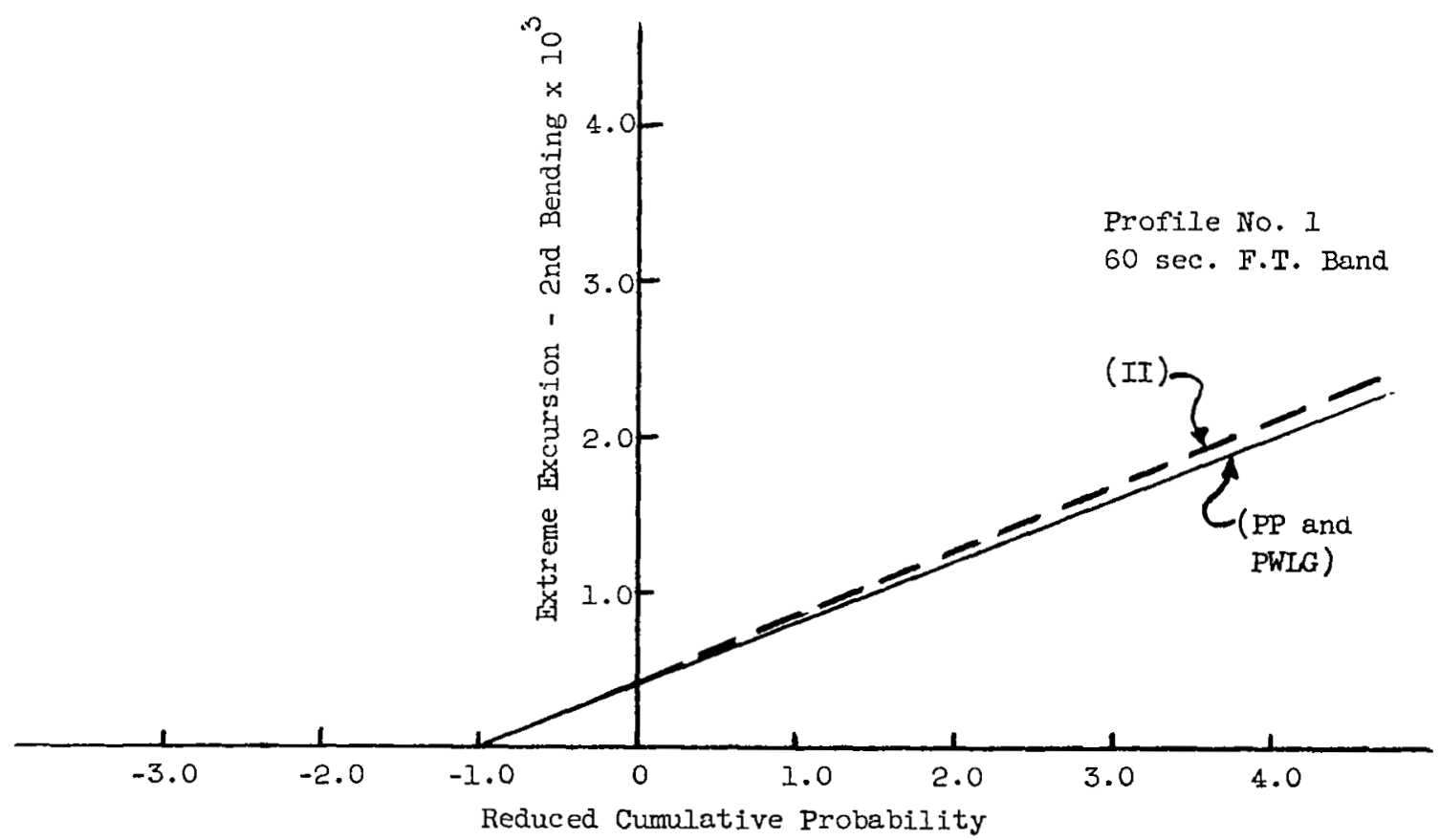

Fig. 48 - Extreme Excursion of 2nd Bending vs. Reduced Cumulative Probability - $60 \mathrm{sec}$. F.T. 


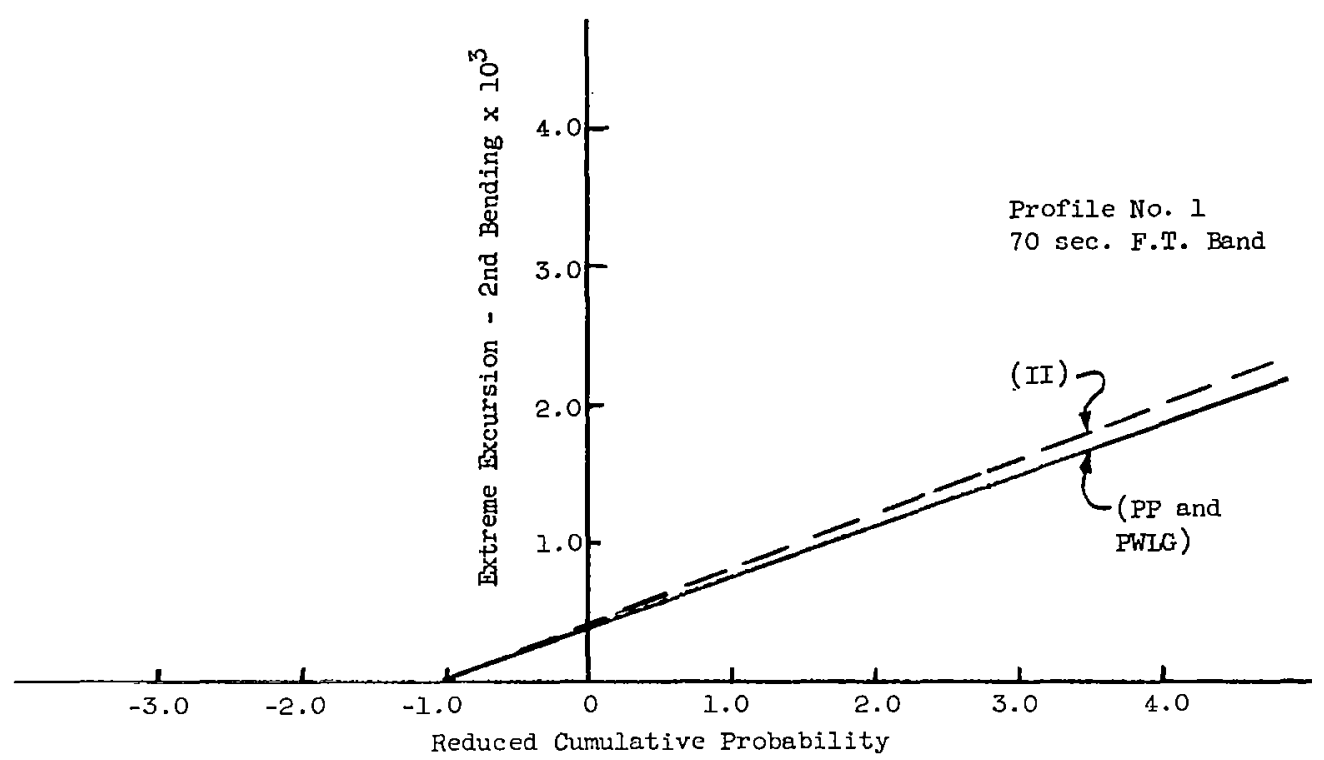

Fig. A9 - Eatreme Excursion of 2nd Bending vs. Reduced Cumulative Probability - $70 \mathrm{sec}$. F.T.

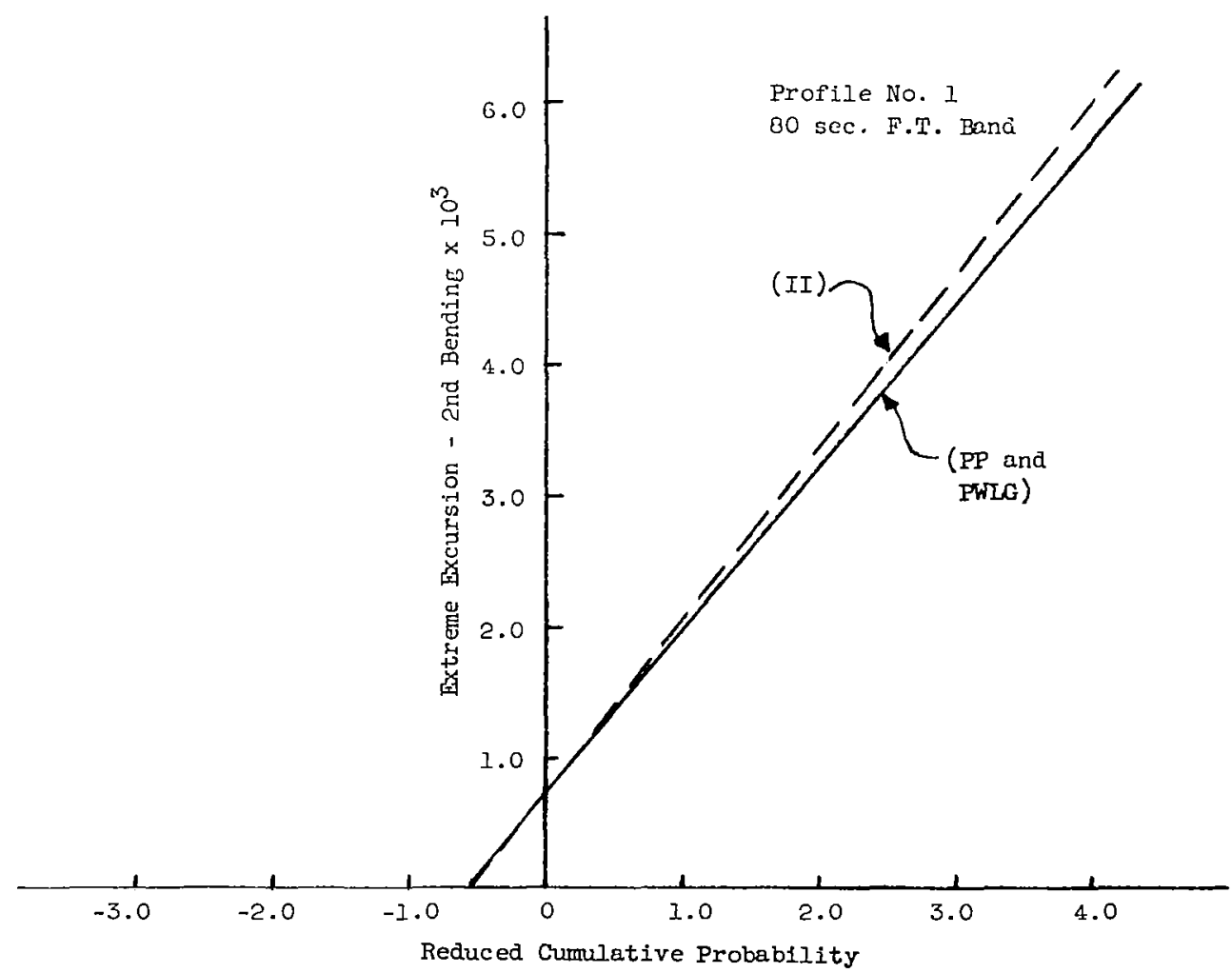

Fig. 50 - Extreme Excursion of and Bending vs. Reduced Cumulative Probability - $80 \mathrm{sec}$. F.T. 


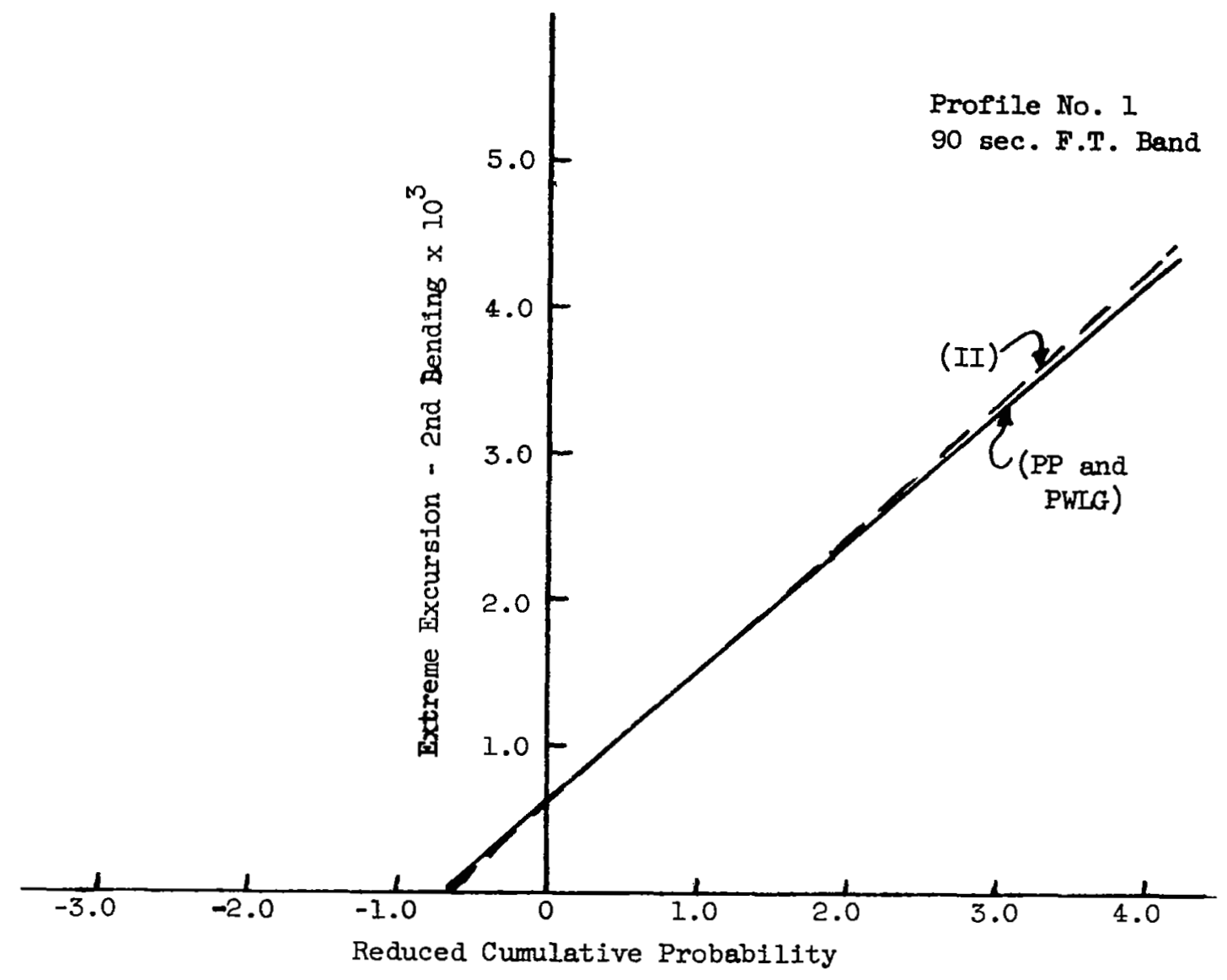

Fig. 51 - Extreme Excursion of 2nd Bending vs. Reduced Cumulative Probability - $90 \mathrm{sec}$. F.T. 


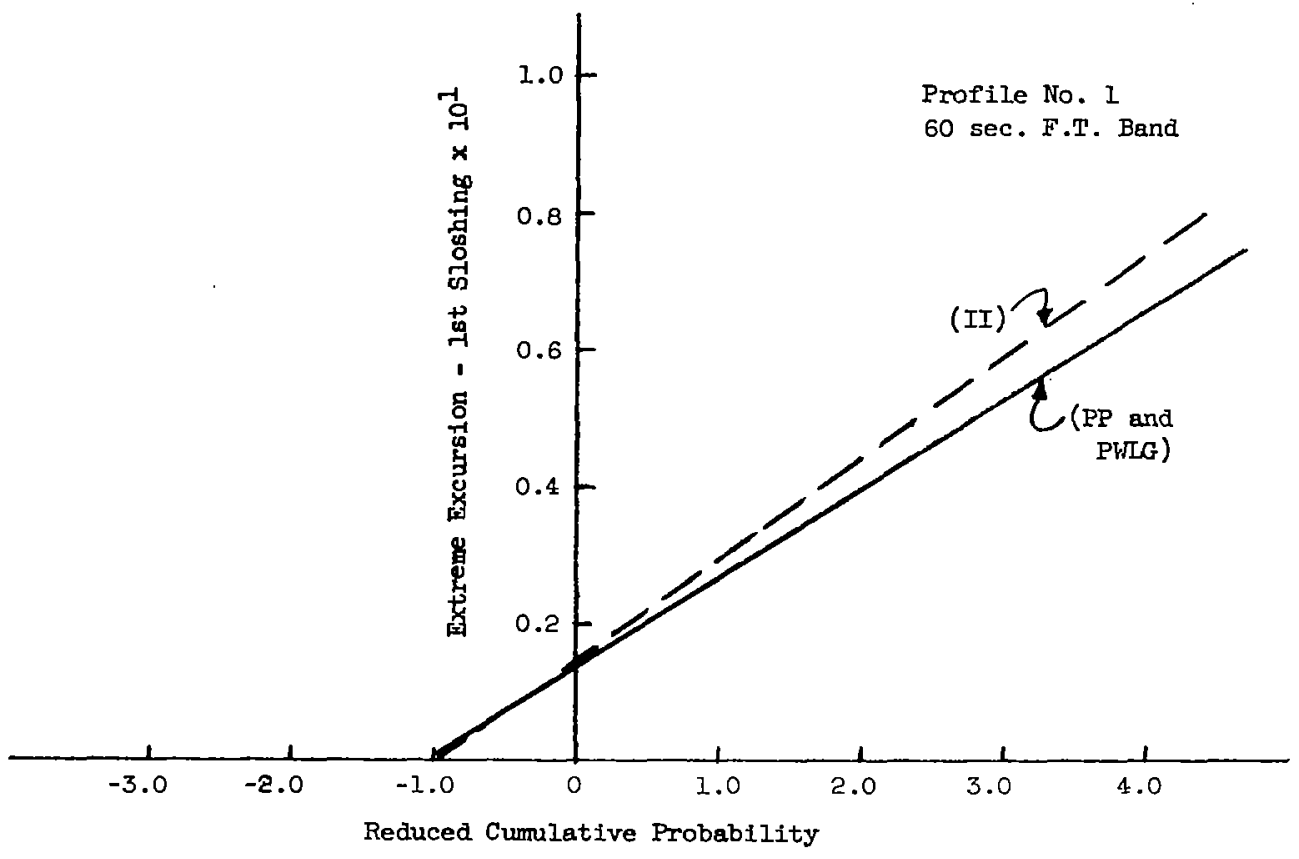

Fig. 52 - Extreme Excursion of lst Sloshing vs. Reduced Cumulative Probability - $60 \mathrm{sec}$. F.T.

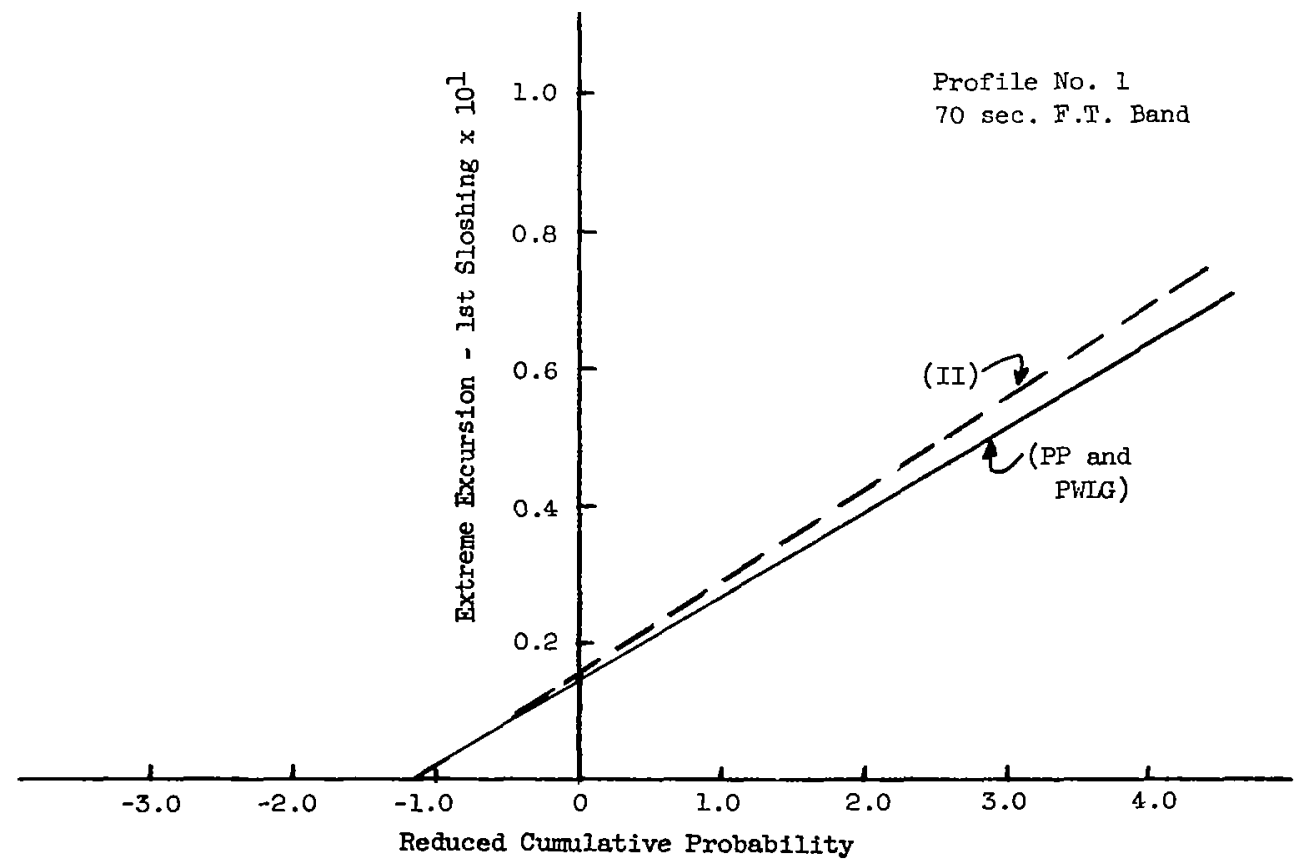

Fig. 53 - Extreme Excursion of lst Sloshing vs. Reduced Cumulative Probability - $70 \mathrm{sec}$. F.T. 


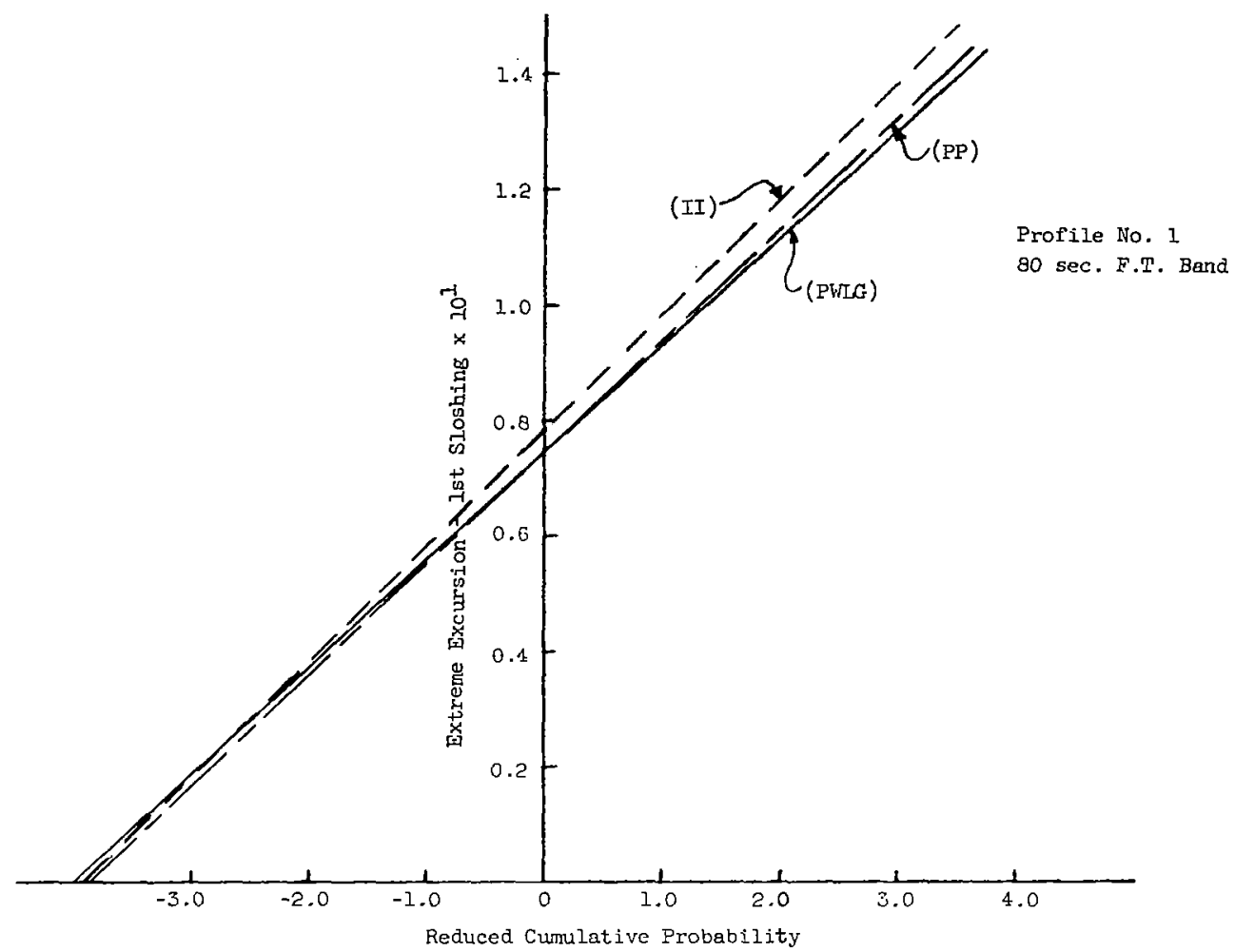

Fig. 54 - Extreme Excursion of Ist Sloshing vs. Reduced Cumulative Probability - $80 \mathrm{sec}$. F.T.

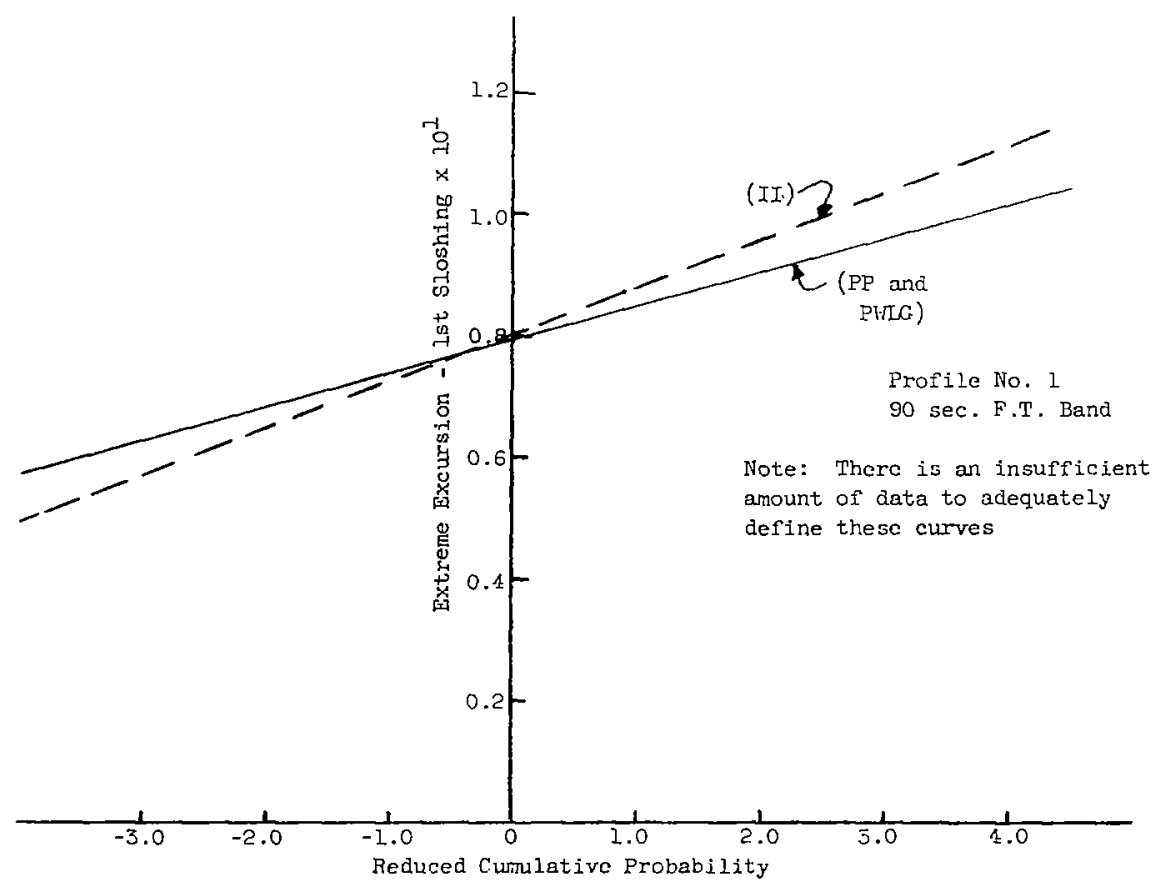

Fig. 55 - Extreme Excursion of 1st Sloshing vs. Reduced Cumulative Probability - $90 \mathrm{sec}$. F.T. 


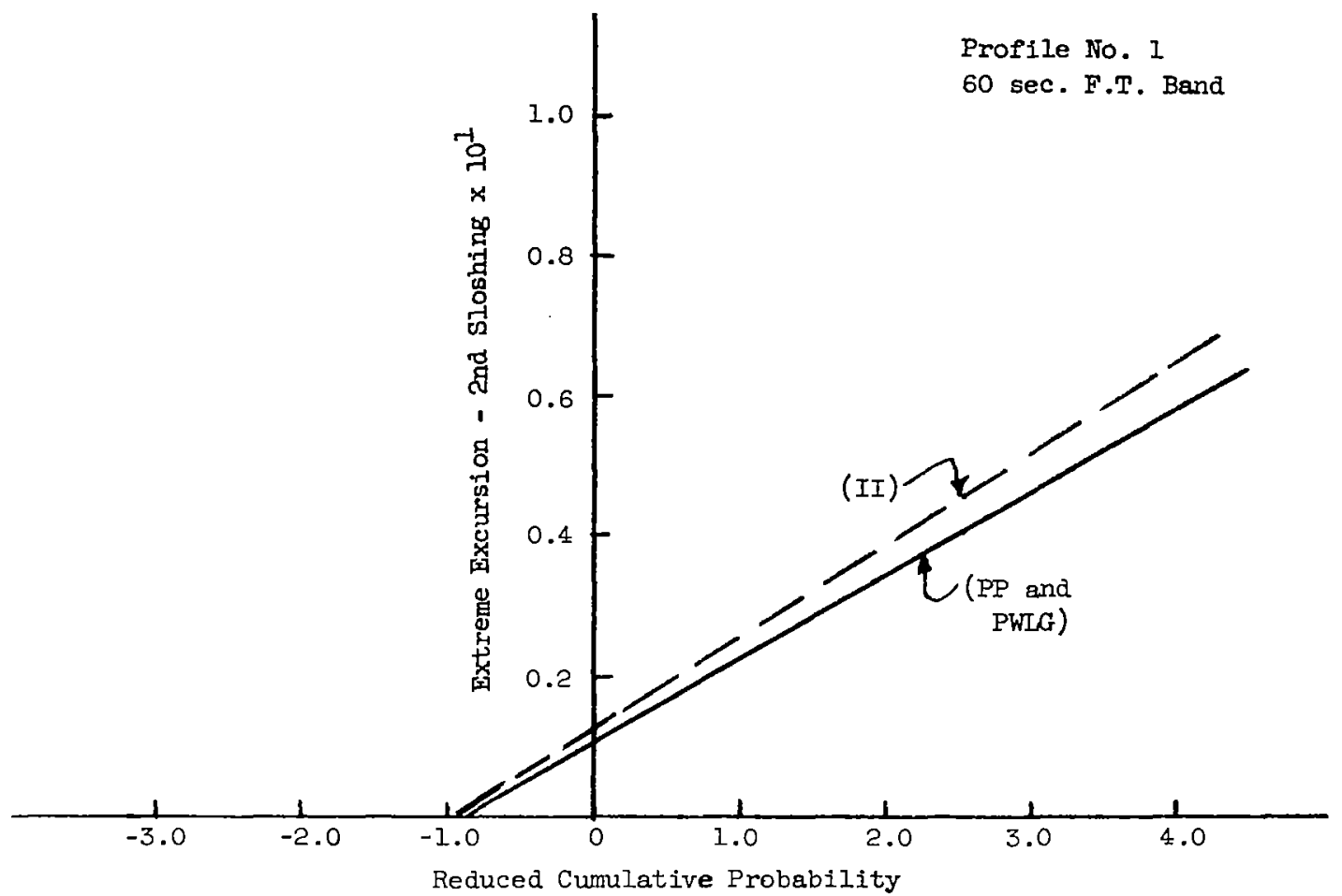

Fig. 56 - Extreme Excursion of 2nd Sloshing vs. Reduced Cumulative Probability - 60 sec. F.T.

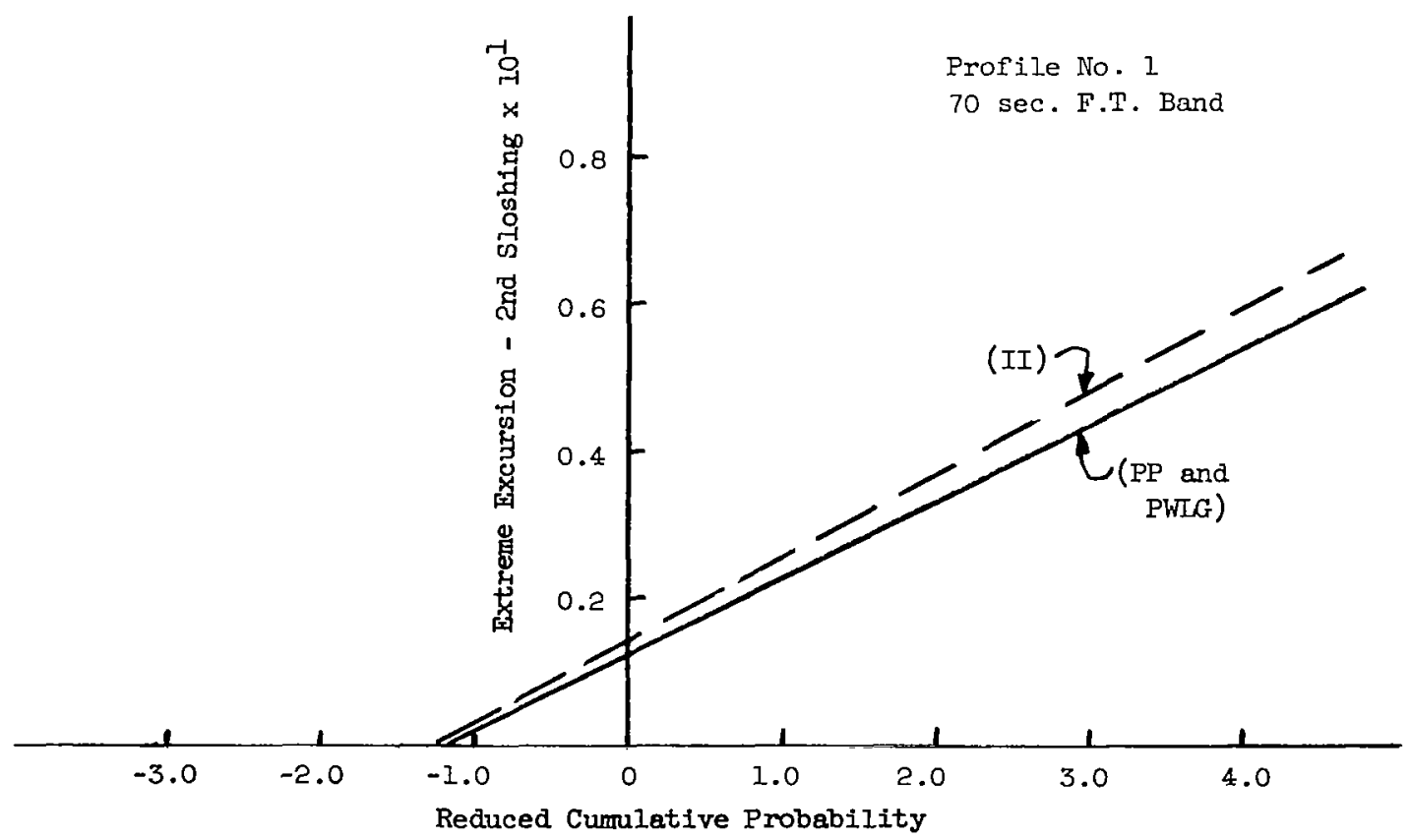

Fig. 57 - Extreme Excursion of 2nd Sloshing vs. Reduced Cumulative Probability - $70 \mathrm{sec}$. F.T. 


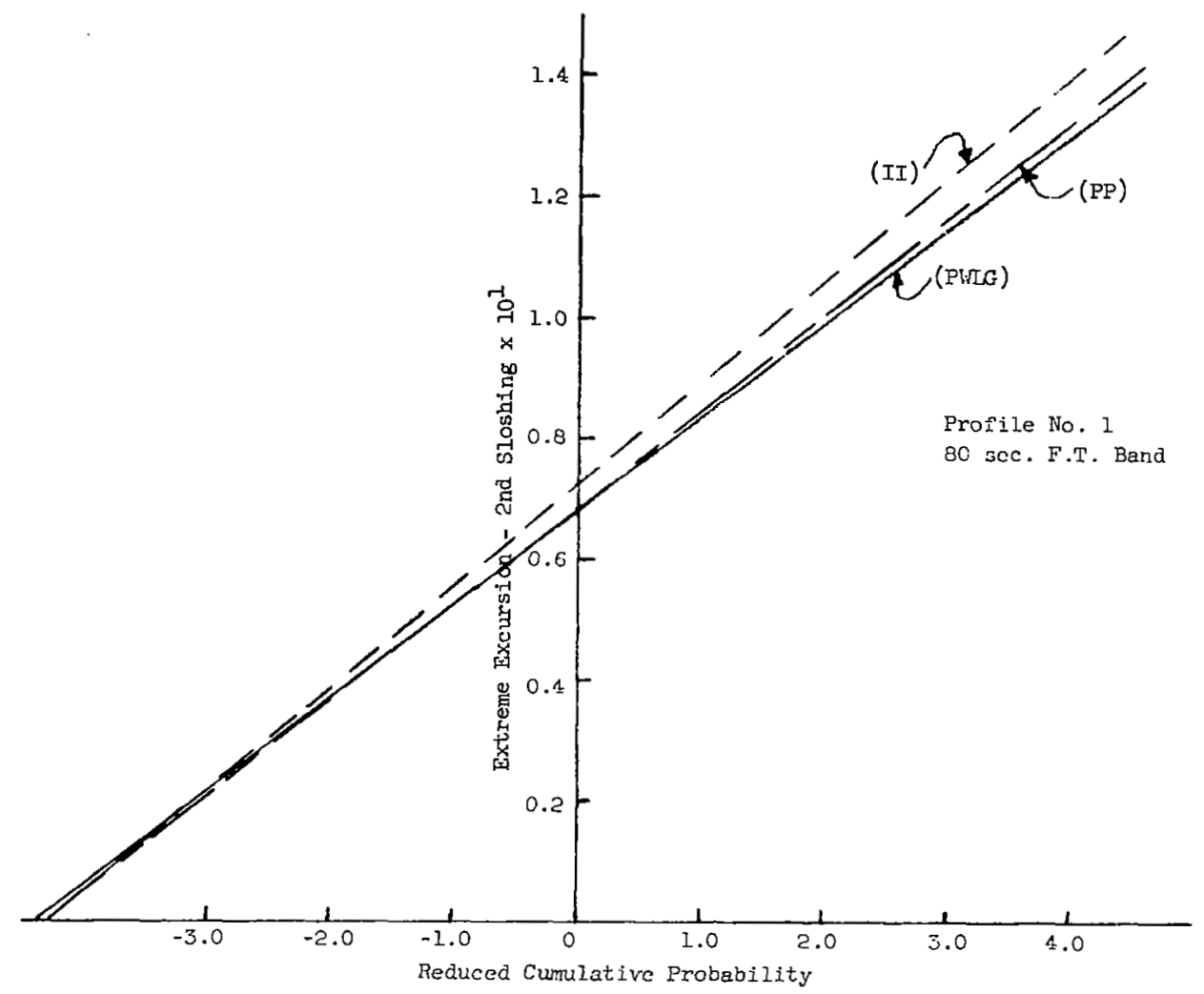

Fig. 50 - Extreme Excursion of 2nd Sloshing vs. Reduced Cumulative Probability - $80 \mathrm{sec}$. F.T.

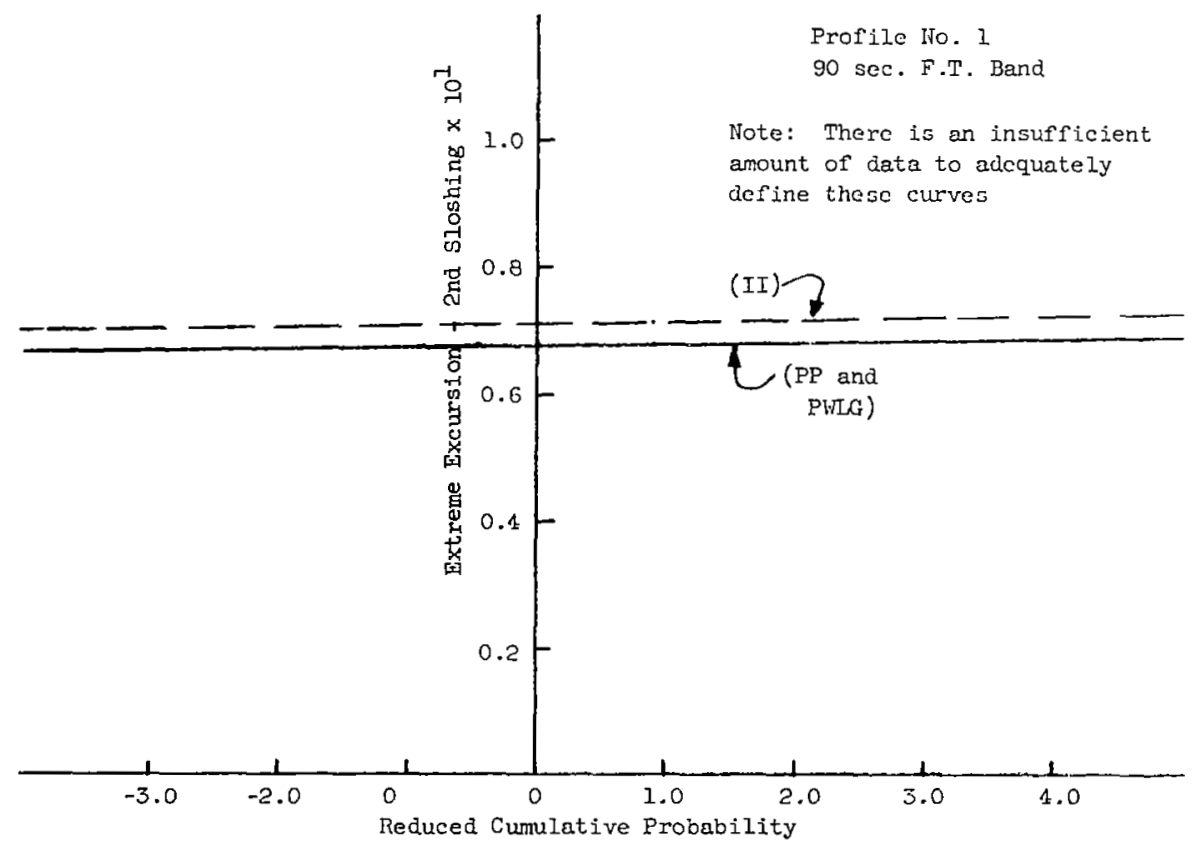

Fig. 59 - Extreme Excursion of and Sloshing vs. Reduced Cumulative Probability - $90 \mathrm{sec}$. F.T. 


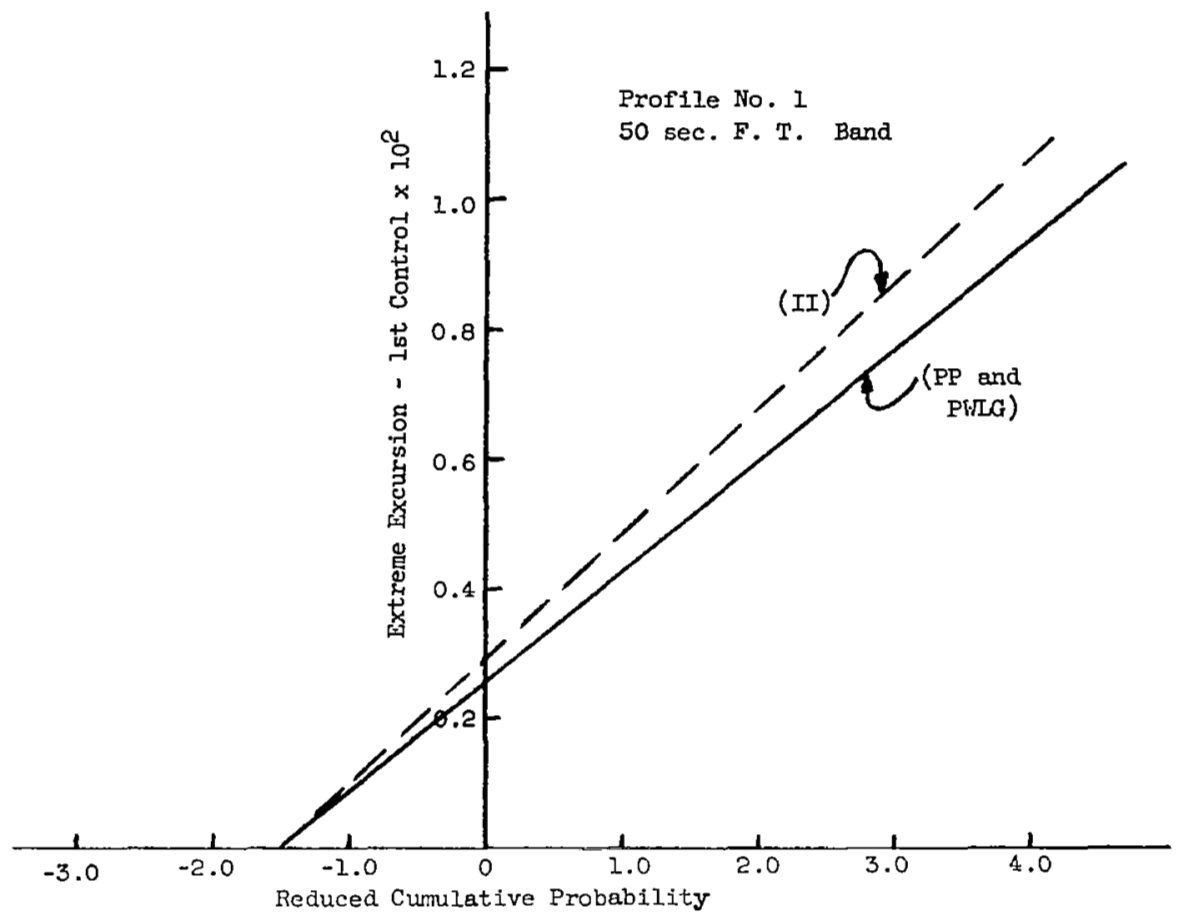

Fig. 30 - Extreme Excursion of Ist Control vs. Reduced Cumulative Probability - $50 \mathrm{sec}$. T.T.

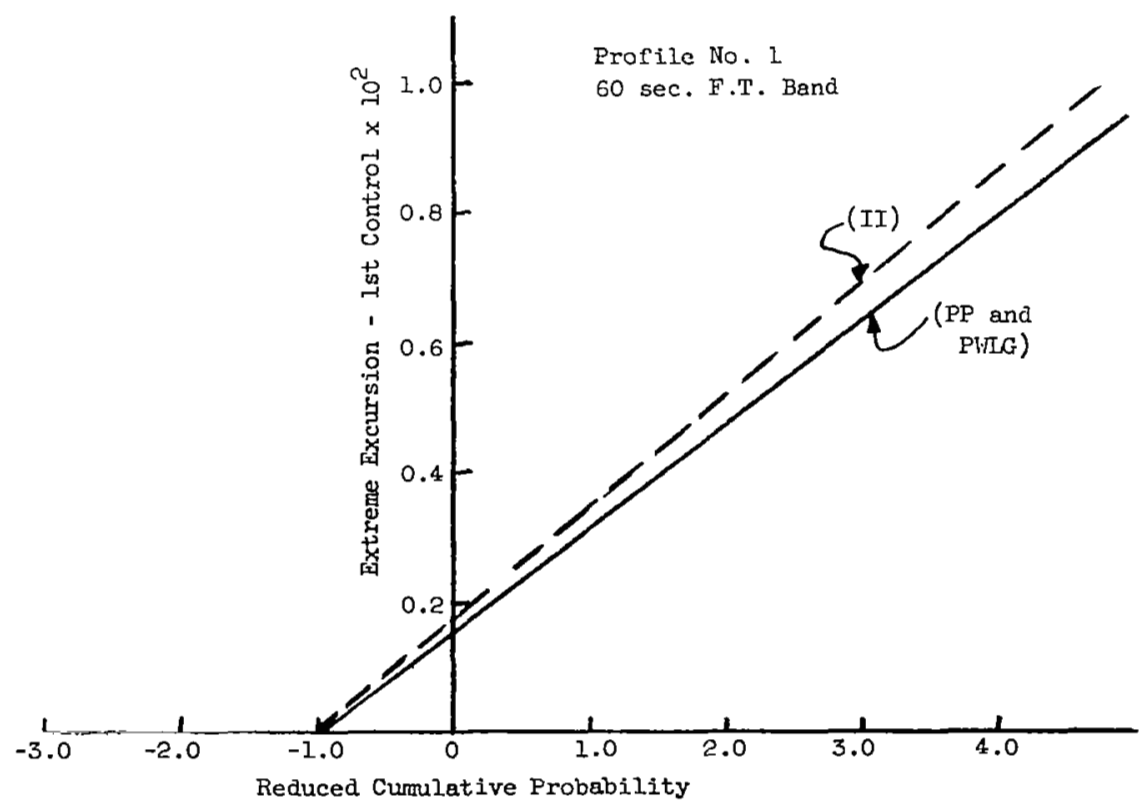

Fig. 61 - Extreme Excursion of lst Control vs. Reduced Cumulative Probebility - $60 \mathrm{sec}$. F.T. 


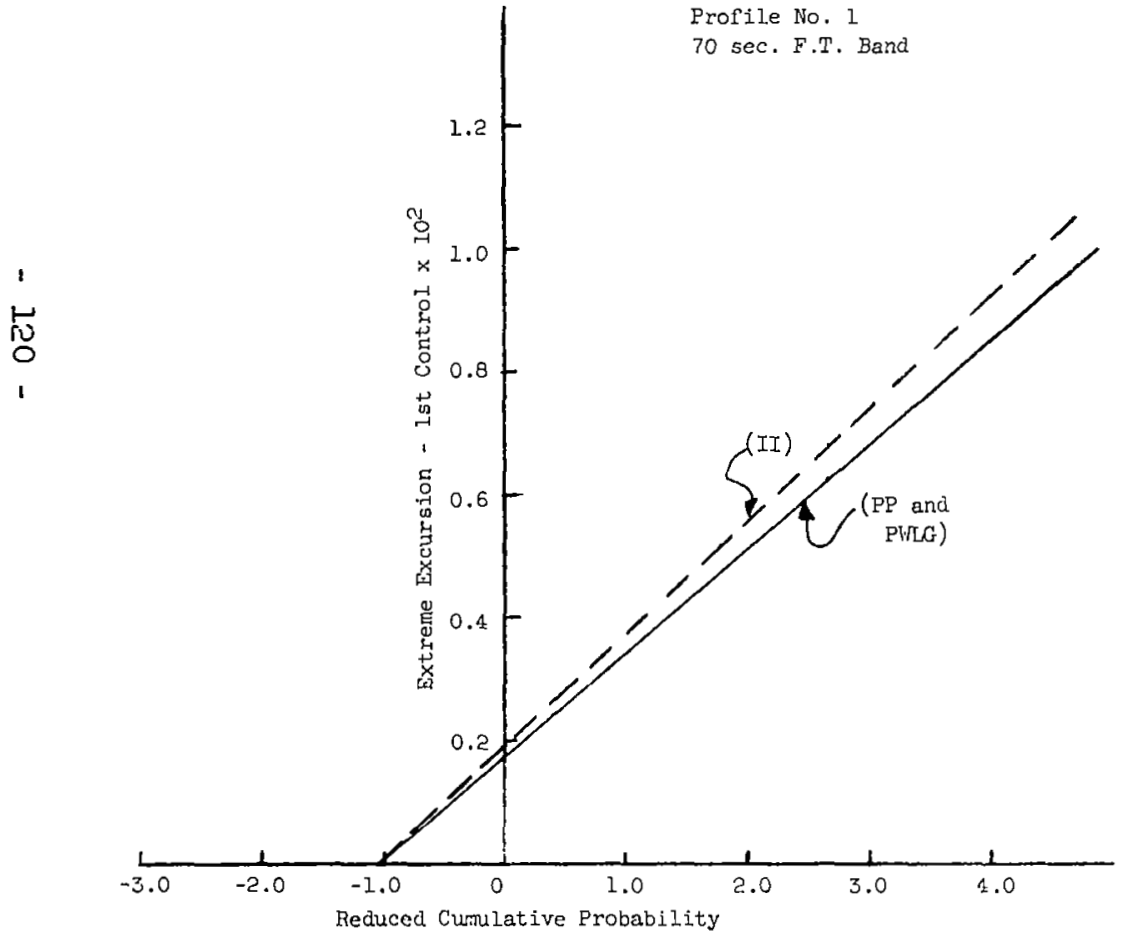

Fig. G2 - Extreme Excursion of 1st Control vs. Reduced Cumulative Probability $-70 \mathrm{sec}$. F.T.

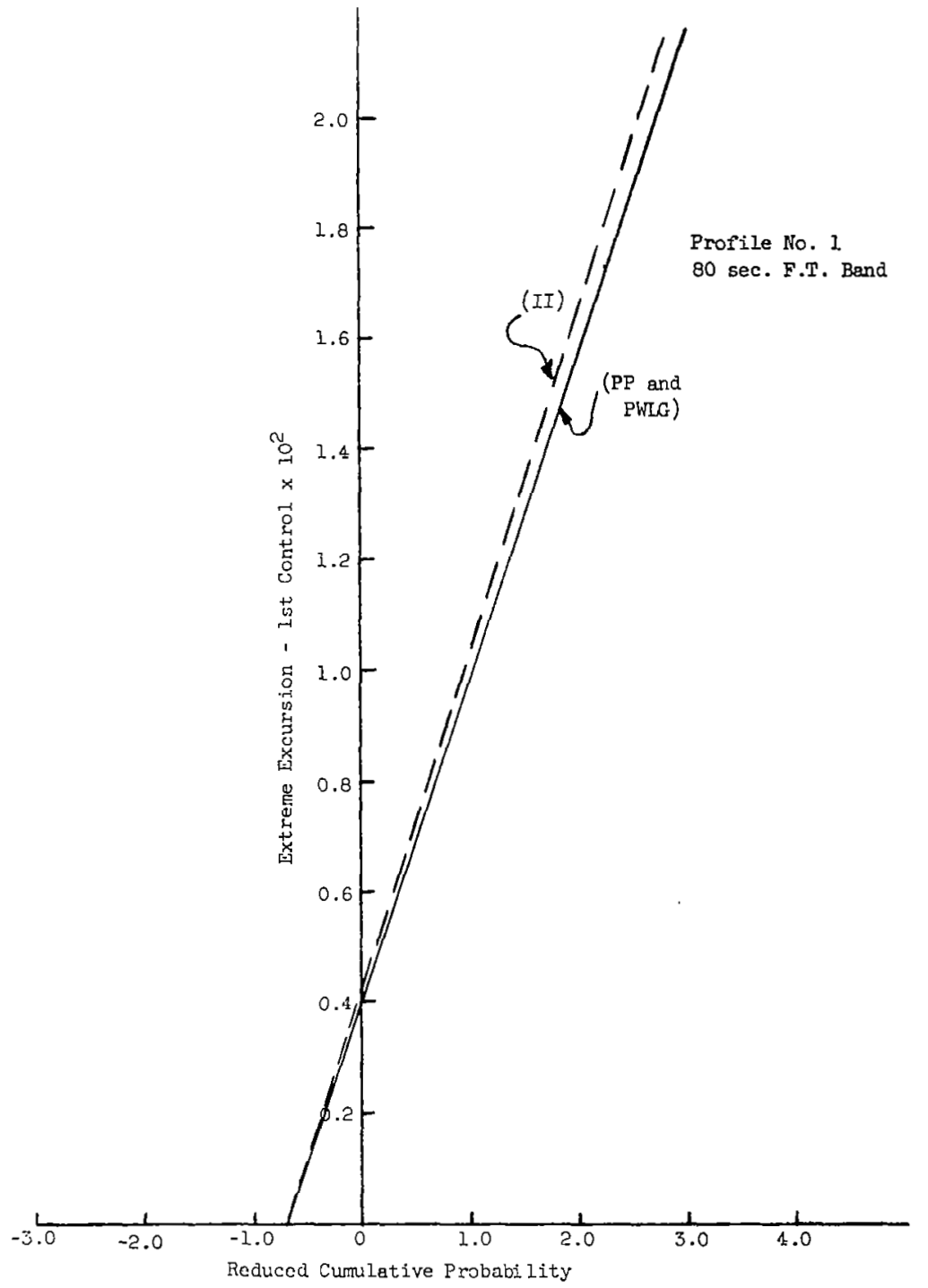

Fig. 63 - Extreme Excursion of lst Control vs. Reduced Cimulative Probability - $80 \mathrm{sec}$. F.T. 


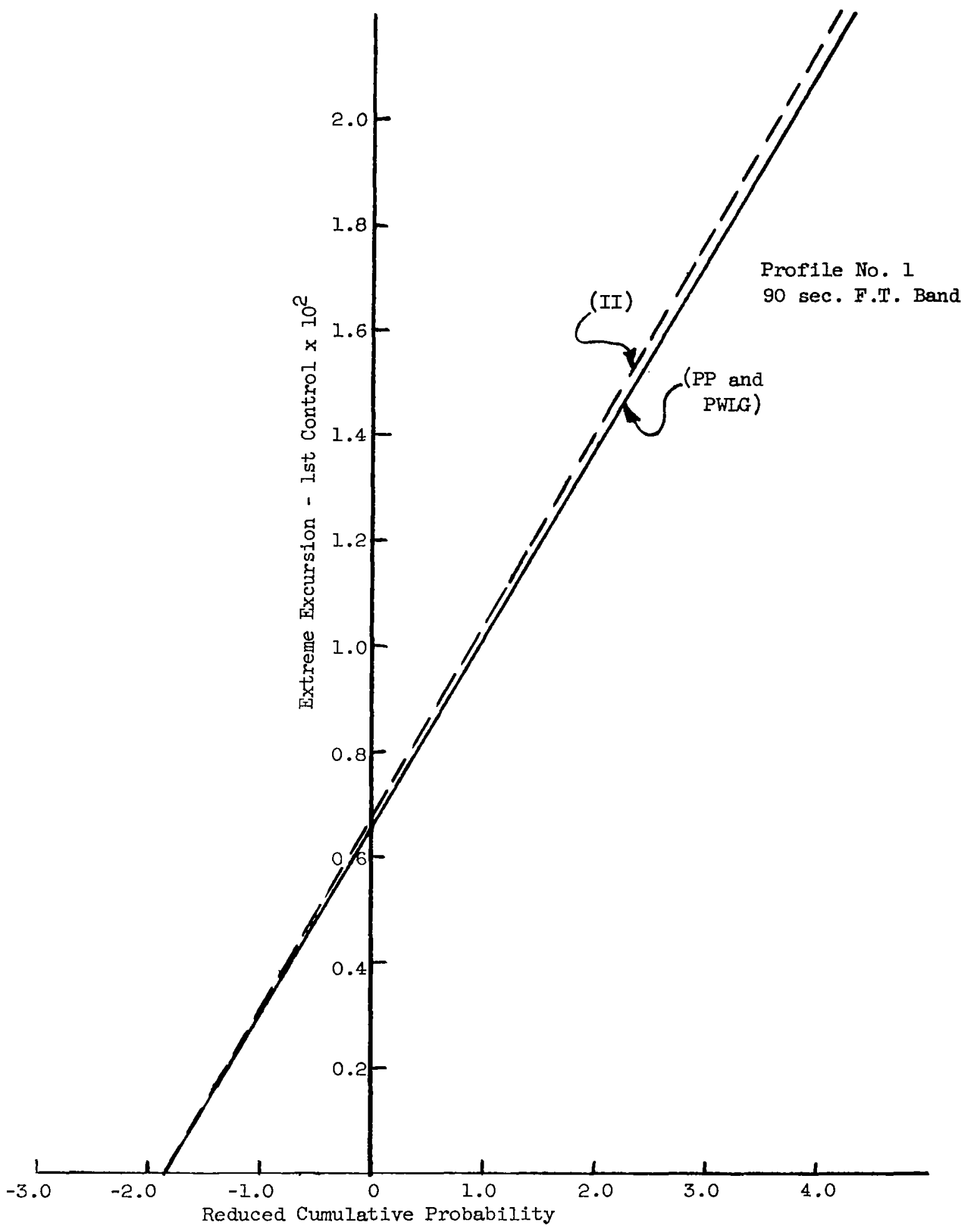

Fig. 64 - Extreme Excursion of lst Control vs. Reduced Cumulative Probability - $90 \mathrm{sec}$. F.T. 


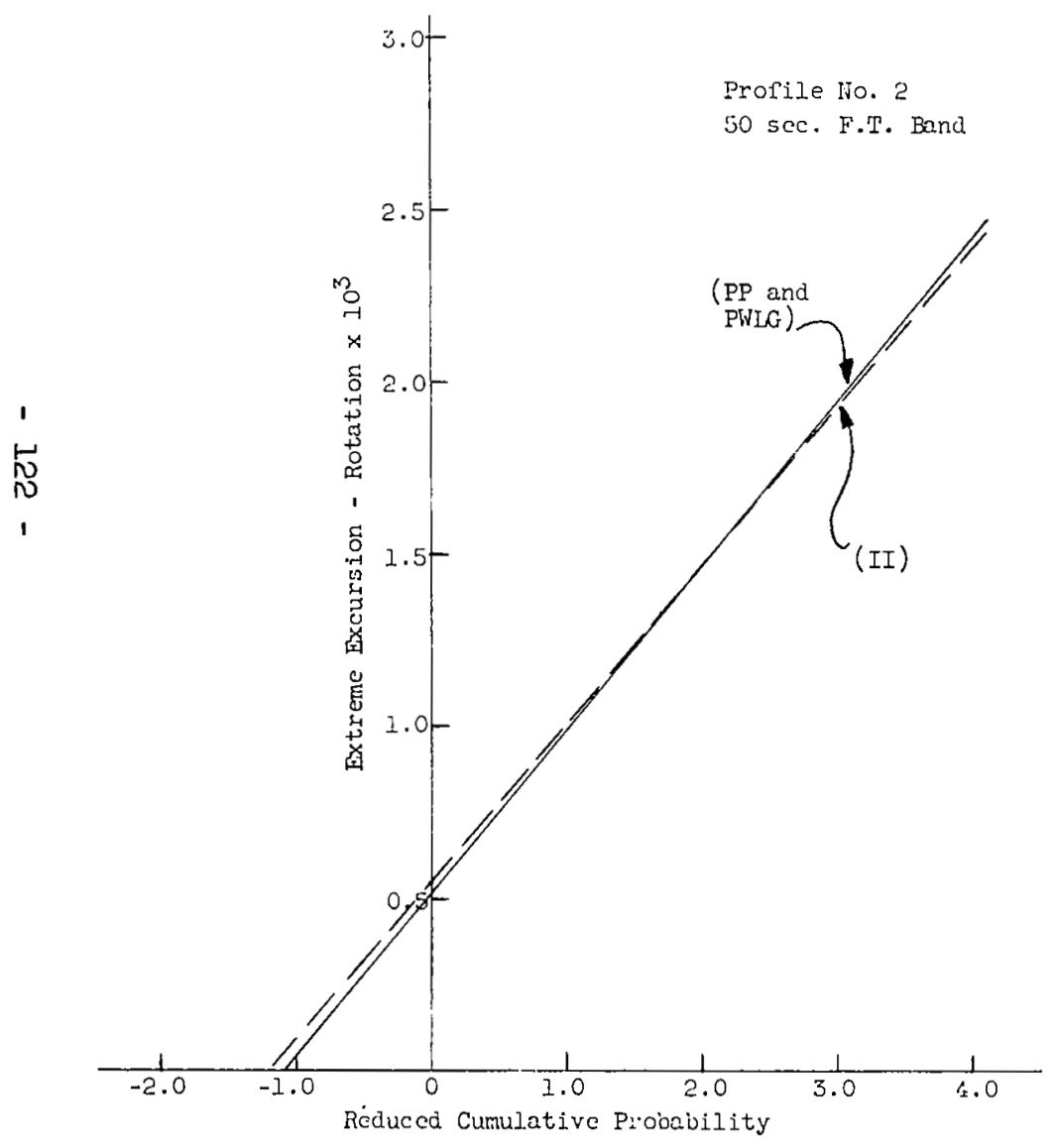

Fig. 65 - Extreme Excursion of Rotation vs. Reduced Cumulative Probability - $50 \mathrm{sec}$. F.T.

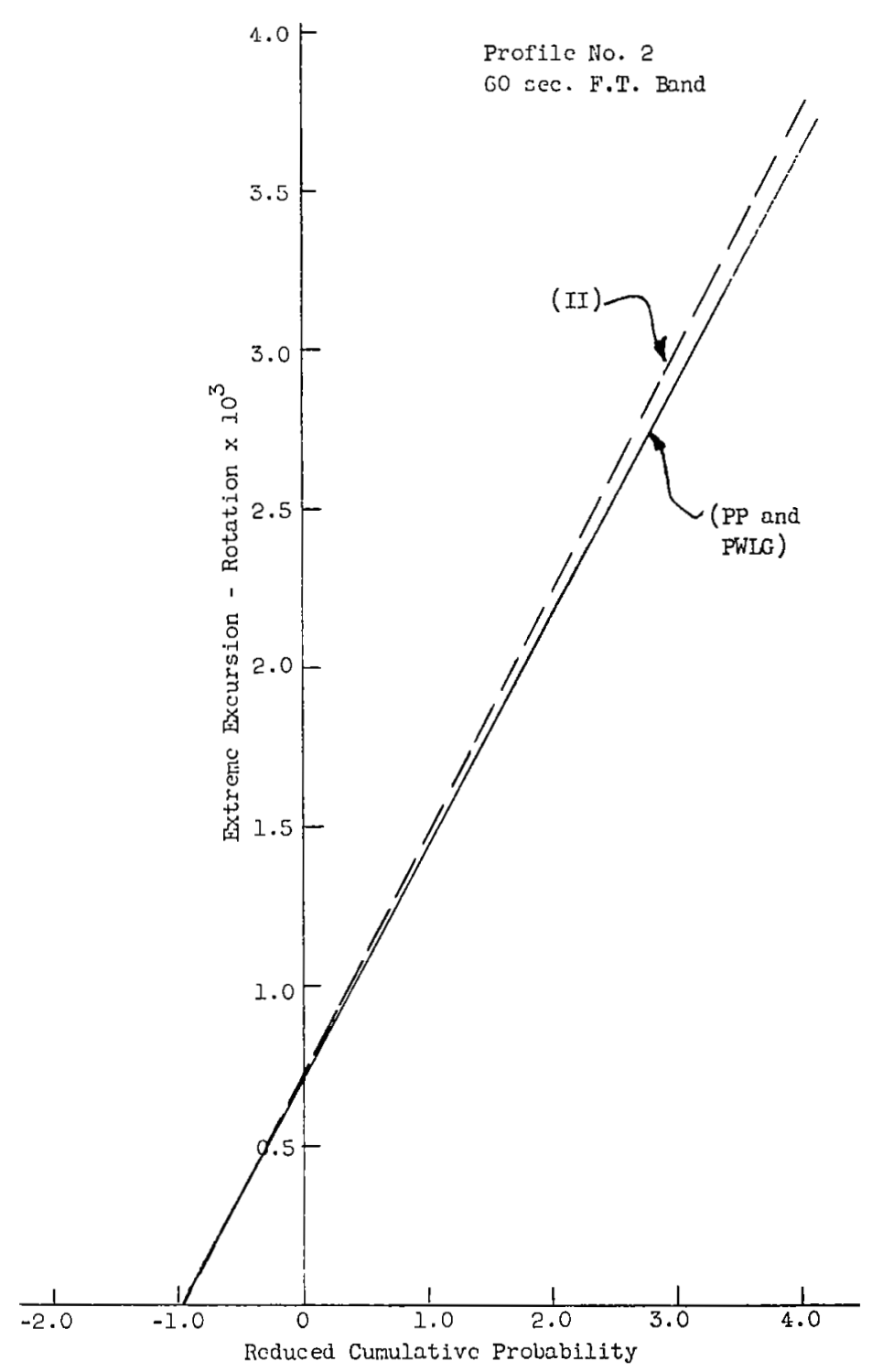

Fig. 66 - Extreme Excursion of Rotation vs. Reduced Cumulative Probability - $60 \mathrm{sec}$. F.T. 


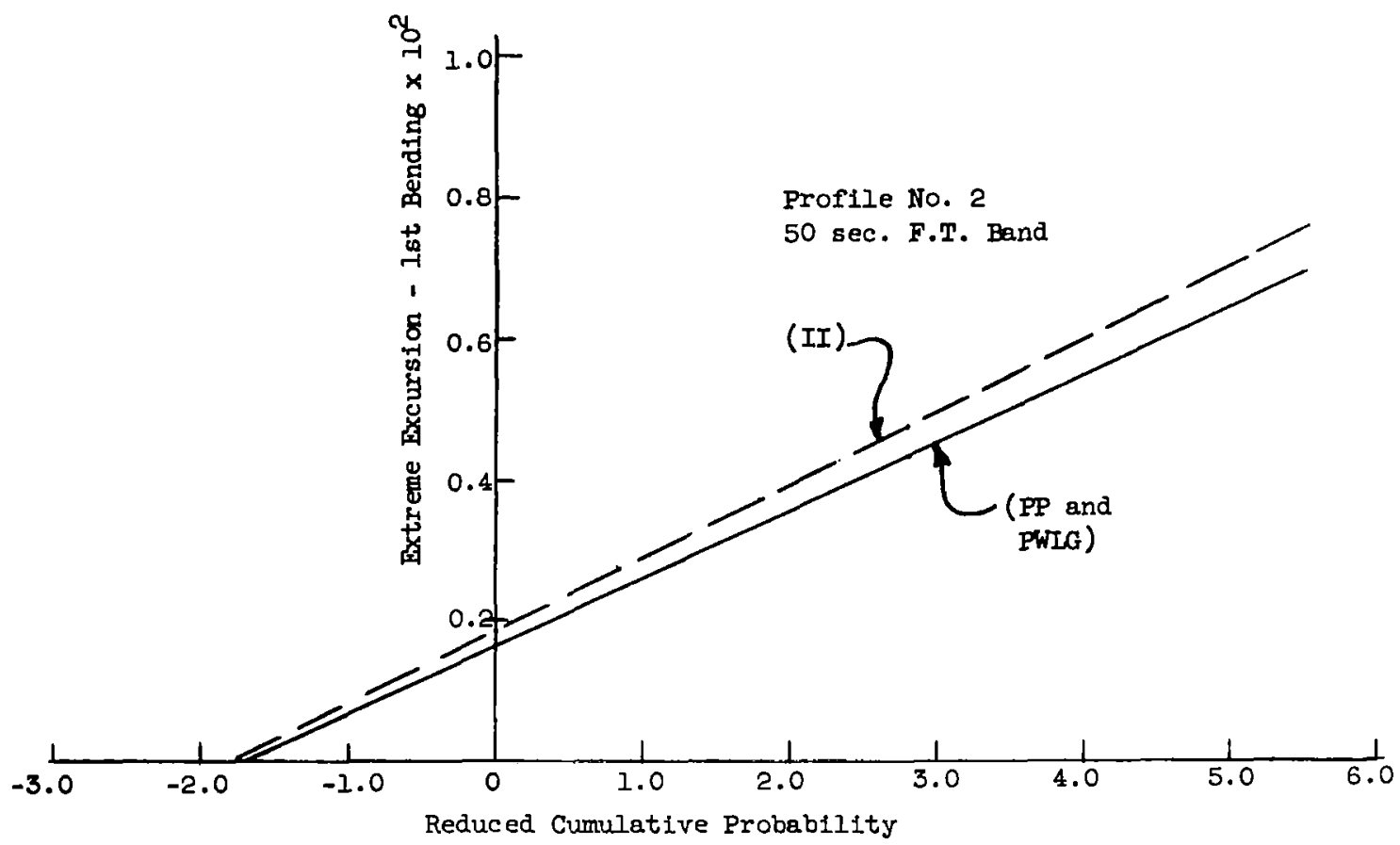

Fig. 67 - Extreme Excursion of lst Bending vs. Reduced Cumulative Probability - $50 \mathrm{sec}$. F.T.

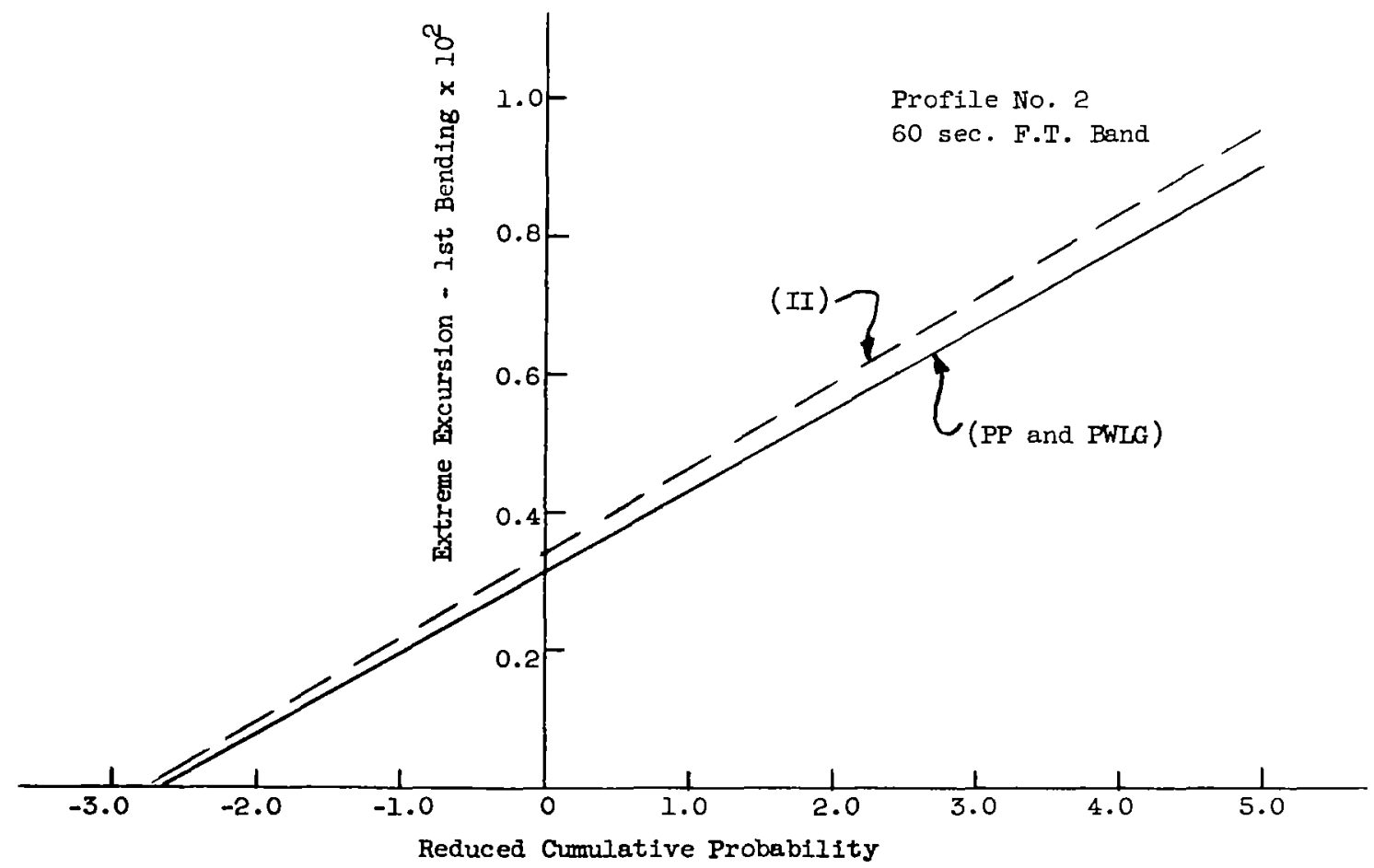

Fig. 68 - Extreme Excursion of lst Bending vs. Reduced Cumulative Probability - $60 \mathrm{sec}$. F.T. 


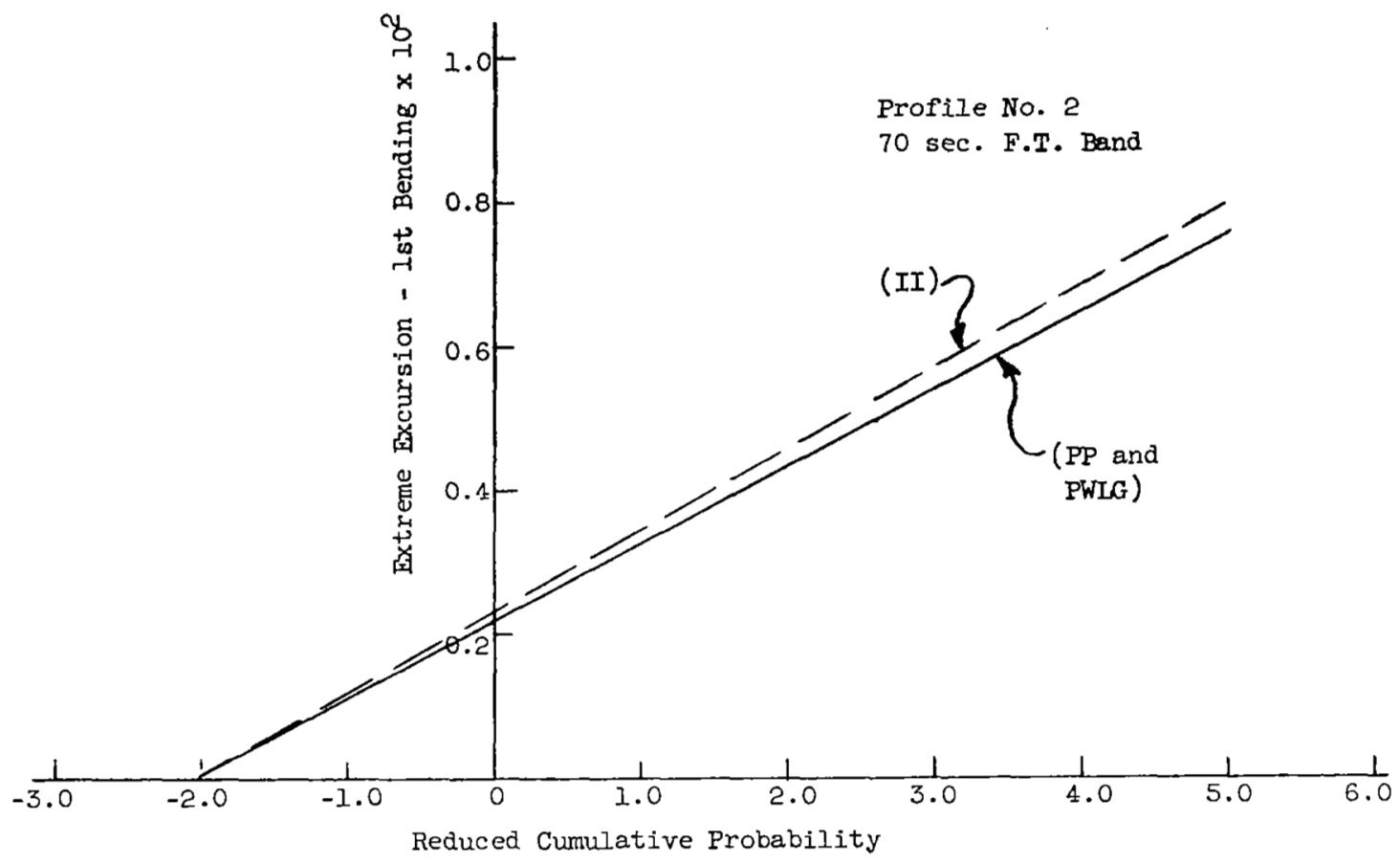

Fig. 69 - Extreme Excursion of lst Bending vs. Reduced Cumulative Probability - $70 \mathrm{sec}$. F.T.

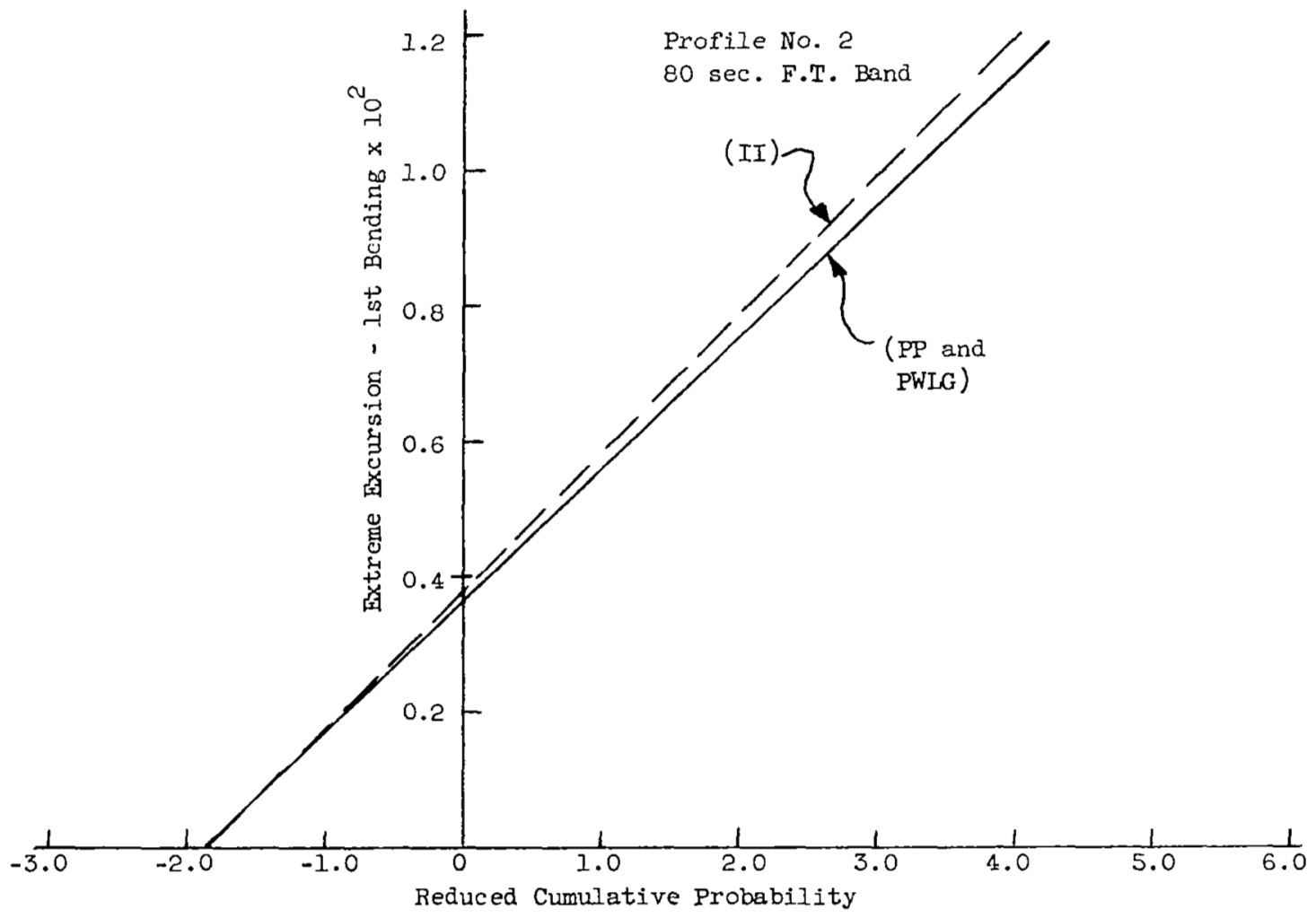

Fig. 70 - Extreme Excursion of lst Bending vs. Reduced Cumulative Probability - $80 \mathrm{sec}$. F.T. 


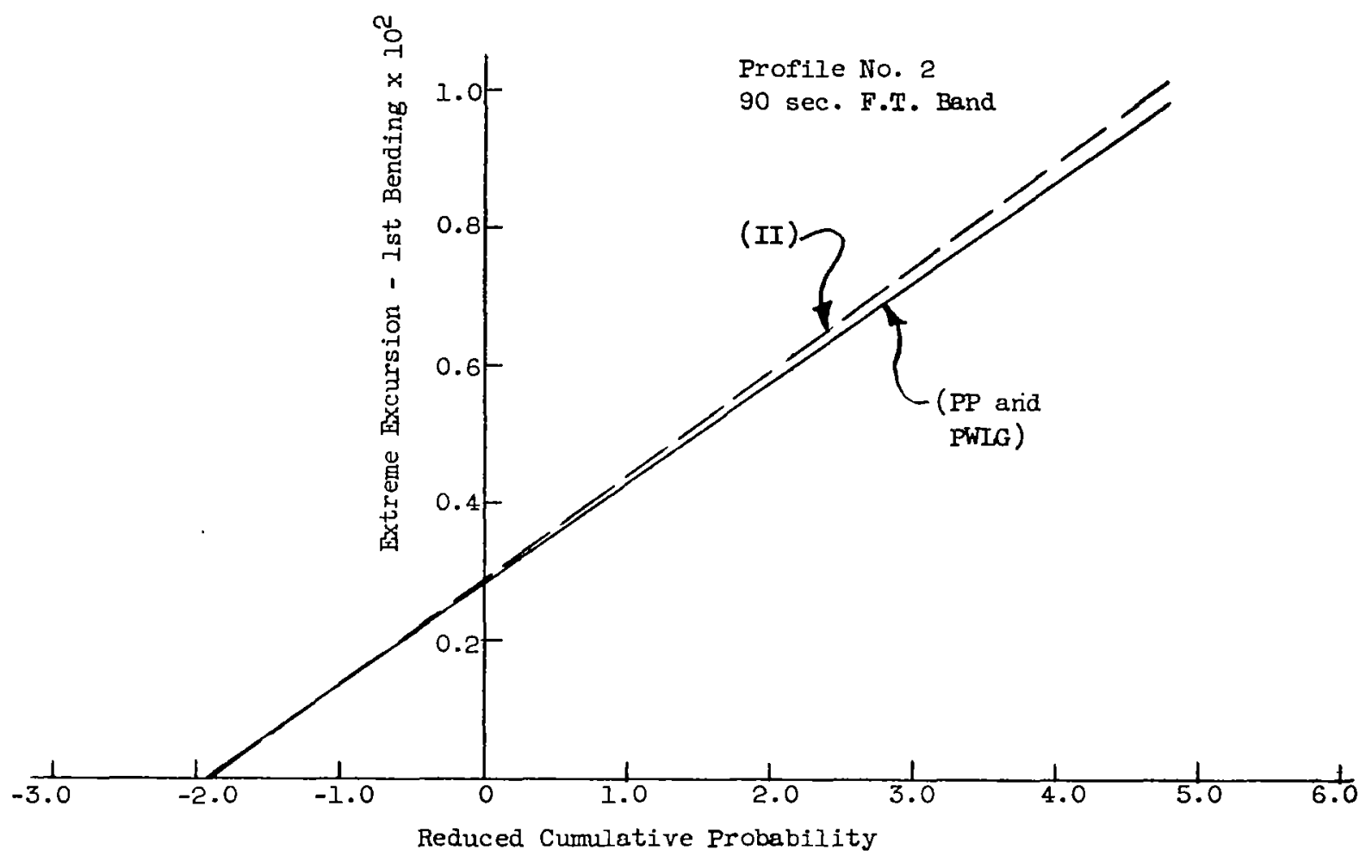

Fig. 71 - Extreme Excursion of lst Bending vs. Reduced Cumulative Probability - $90 \mathrm{sec}$. F.T. 


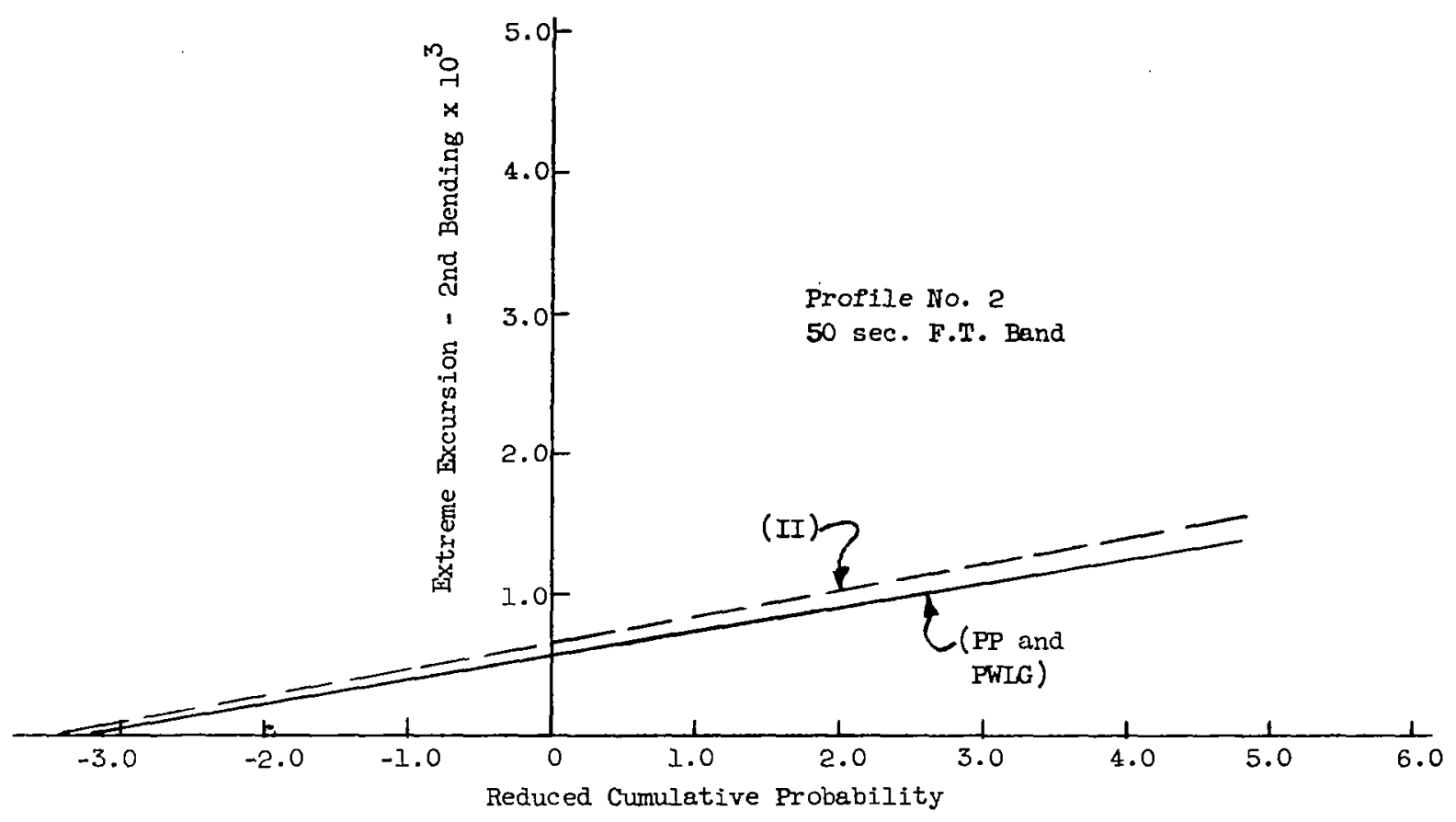

Fig. 72 - Extreme Excursion of 2nd Bending vs. Reduced Cumulative Probability - 50 sec. F.T.

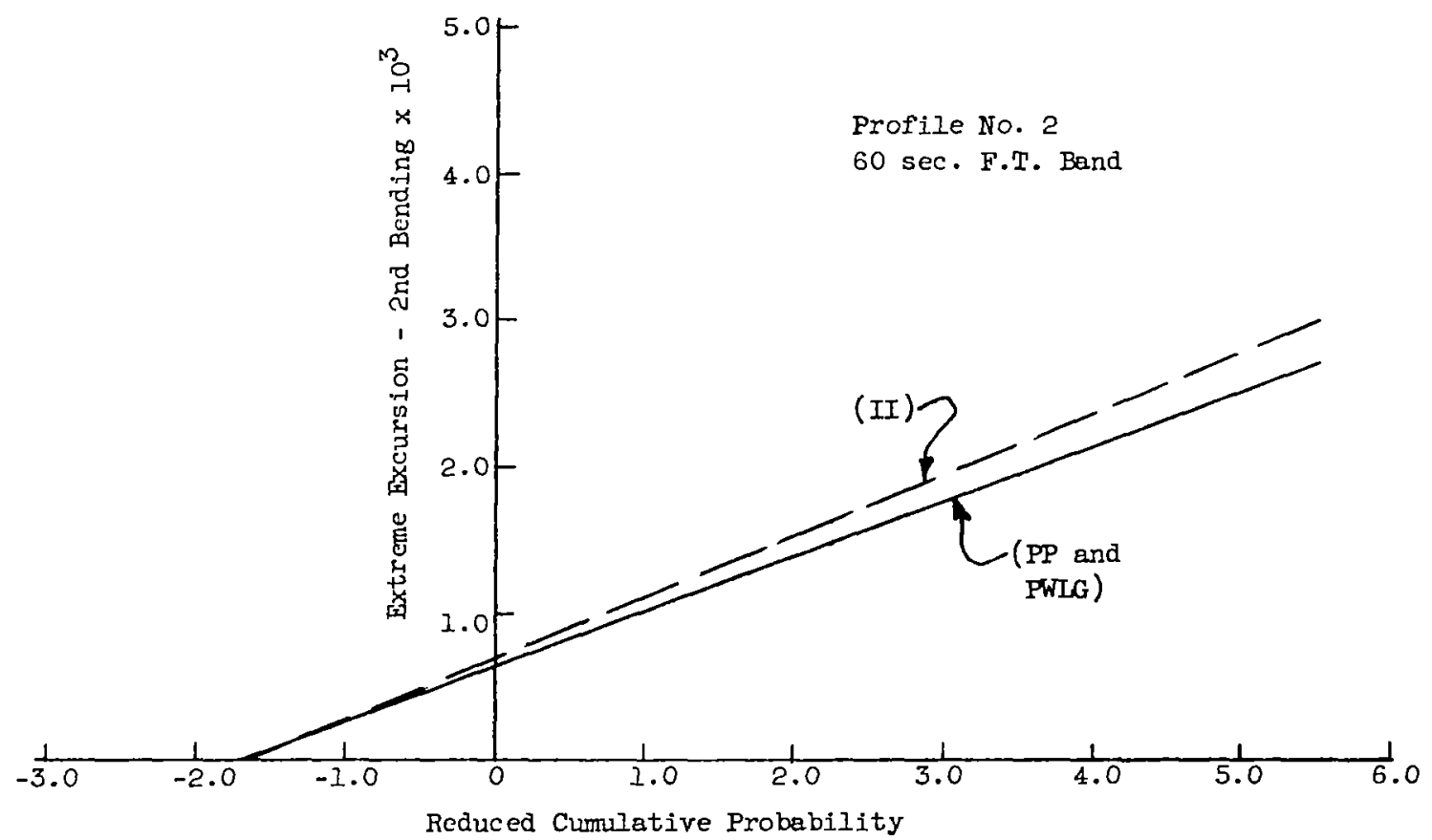

Fig. 73 - Extreme Excursion of 2nd Bending vs. Reduced Cumulative Probability - $60 \mathrm{sec}$. F.T. 


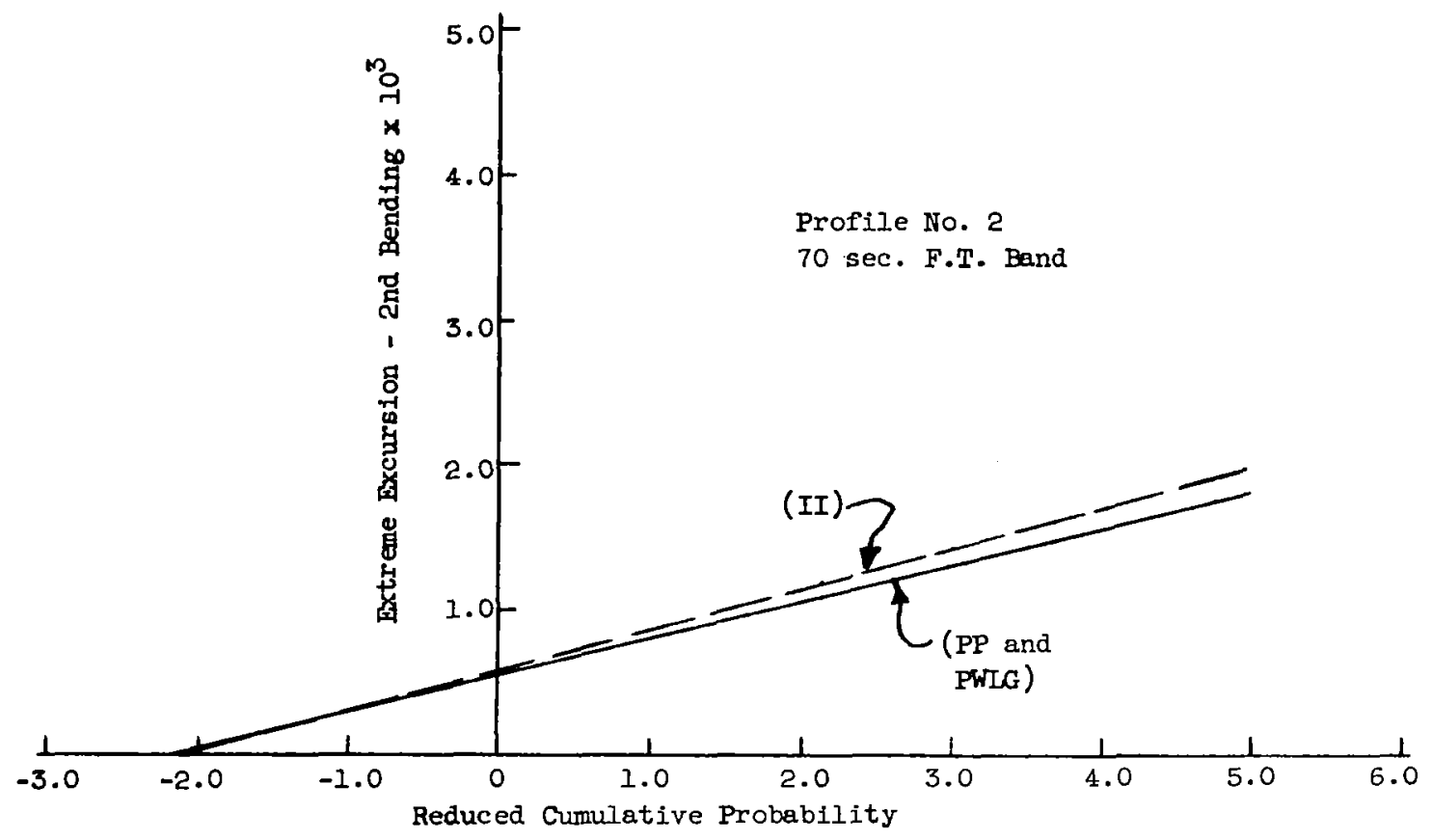

Fig. 74 - Extreme Excursion of 2nd Bending vs. Reduced Cumulative Probability - $70 \mathrm{sec}$. F.T.

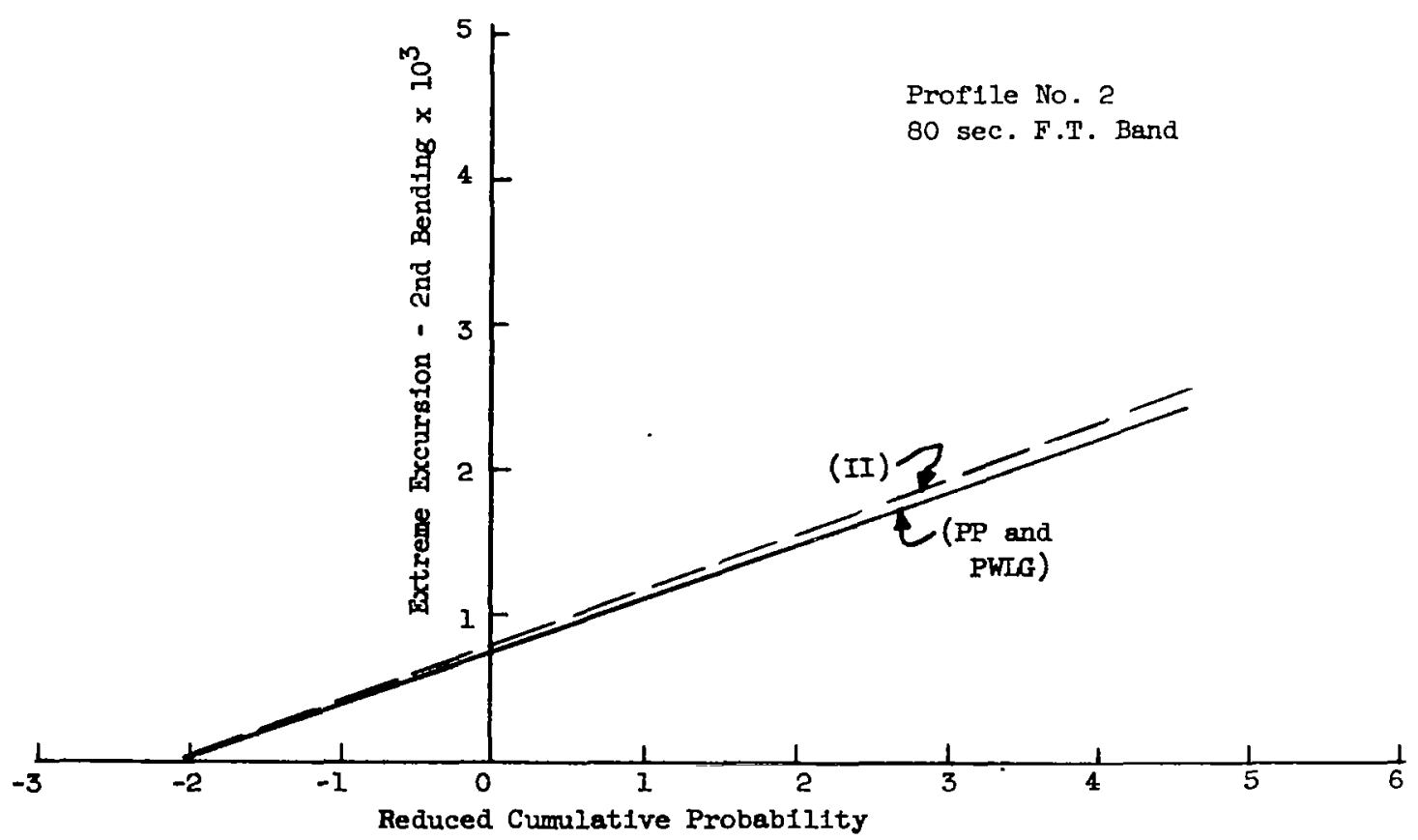

Fig. 75 - Extreme Excursion of 2nd Bending vs. Reduced Cumulative Probability - $80 \mathrm{sec}$. F.T. 


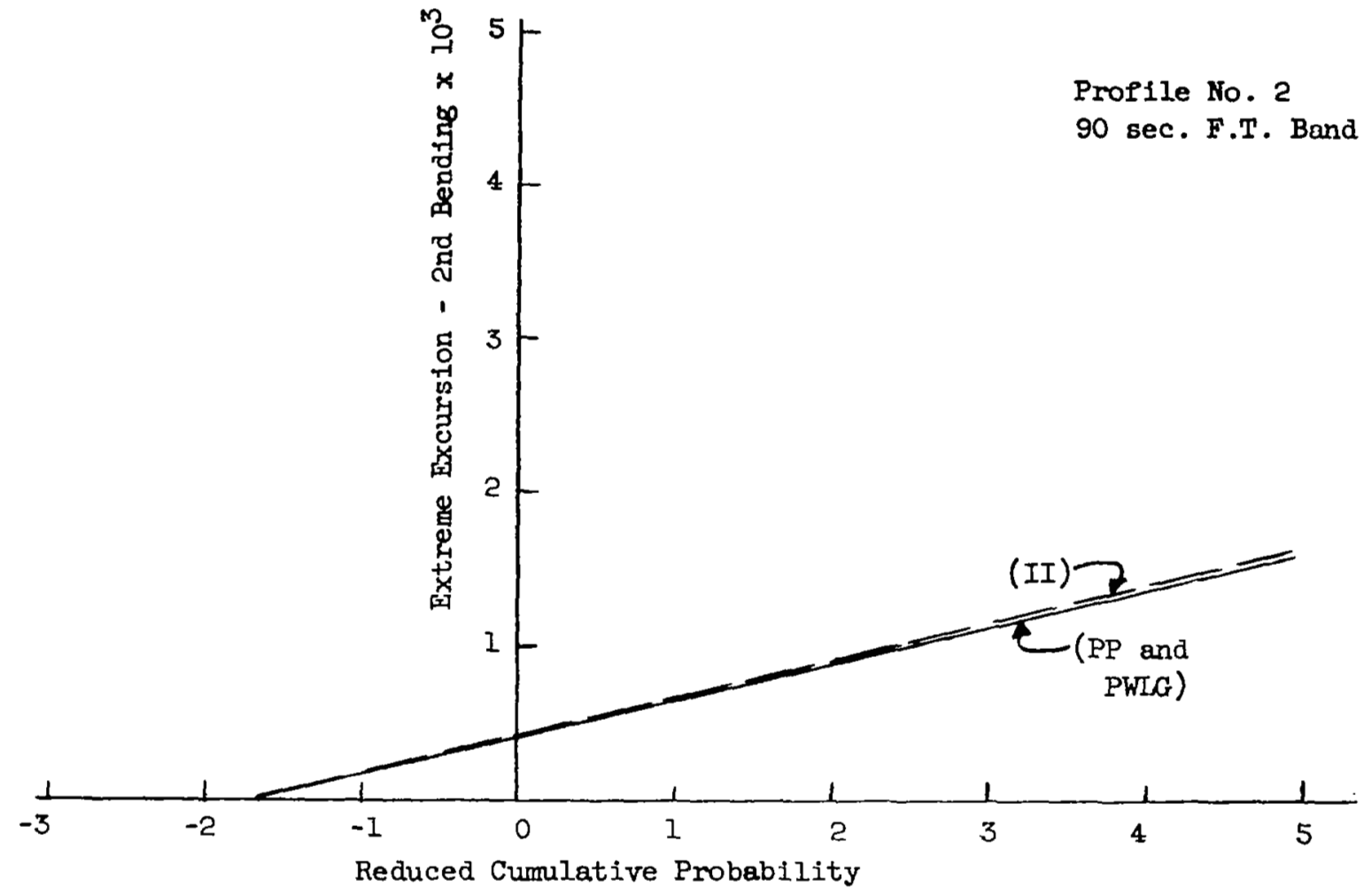

Fig. 76 - Extreme Excursion of and Bending vs. Reduced Cumulative Probability - $90 \mathrm{sec}$. F.T. 


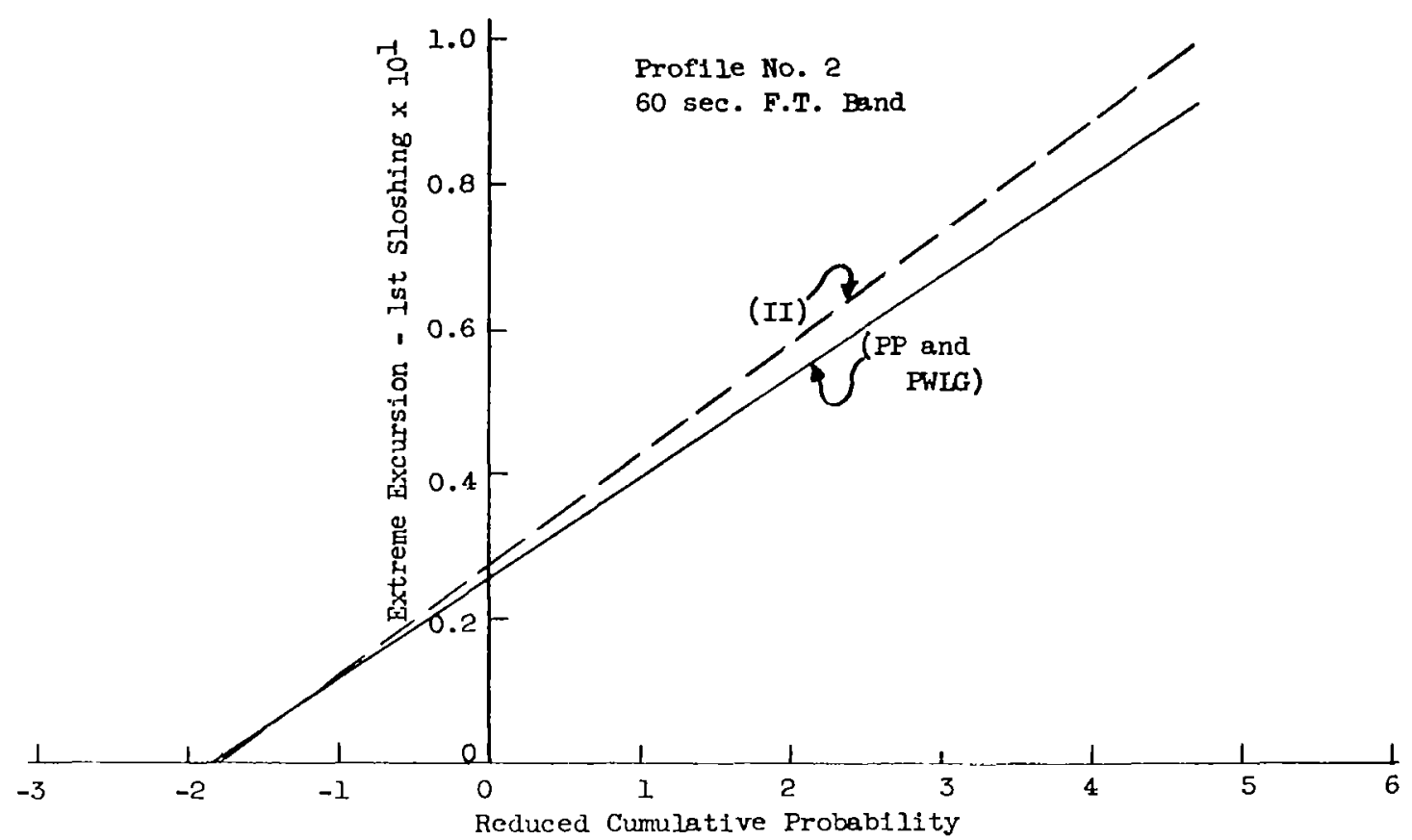

Fig. 77 - Extreme Excursion of lst Sloshing vs. Reduced Cumulative Probability - $60 \mathrm{sec}$. F.T.

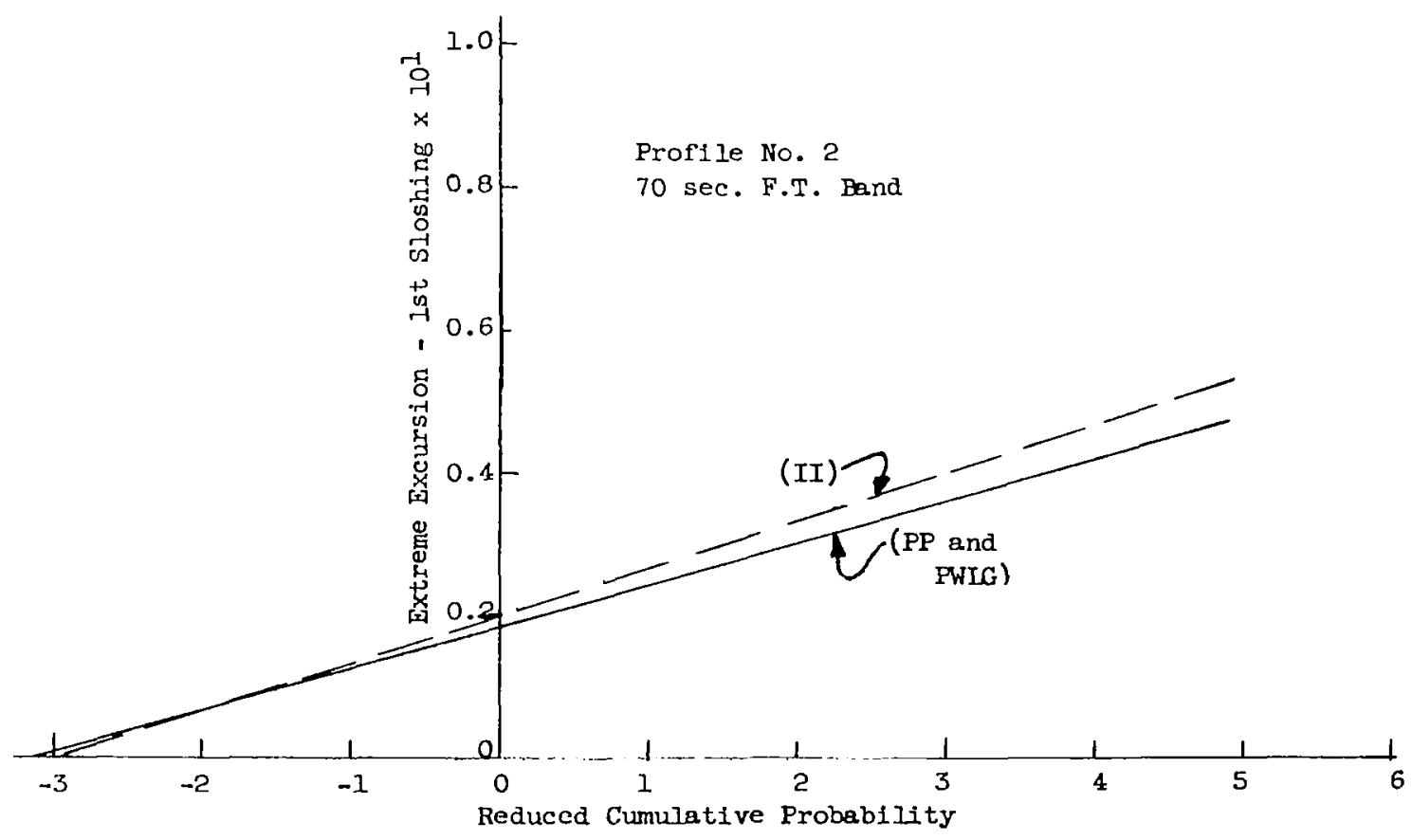

Fig. 78 - Extreme Excursion of lst Sloshing vs. Reduced Cumulative Probability - 70 sec. F.T. 


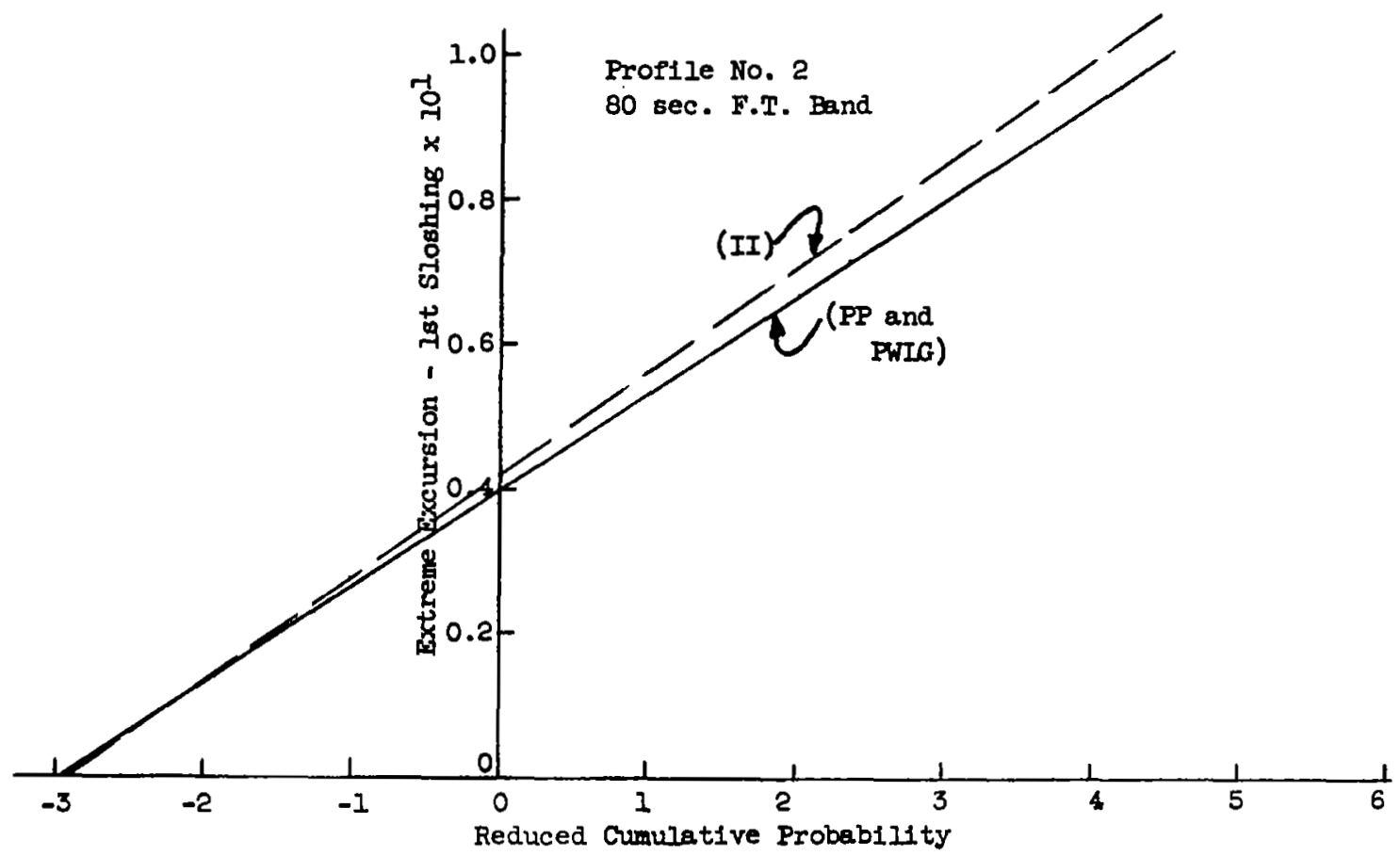

Fig. 79 - Extreme Excursion of lst Sloshing vs. Reduced Cumulative Probability - 80 sec. F.T.

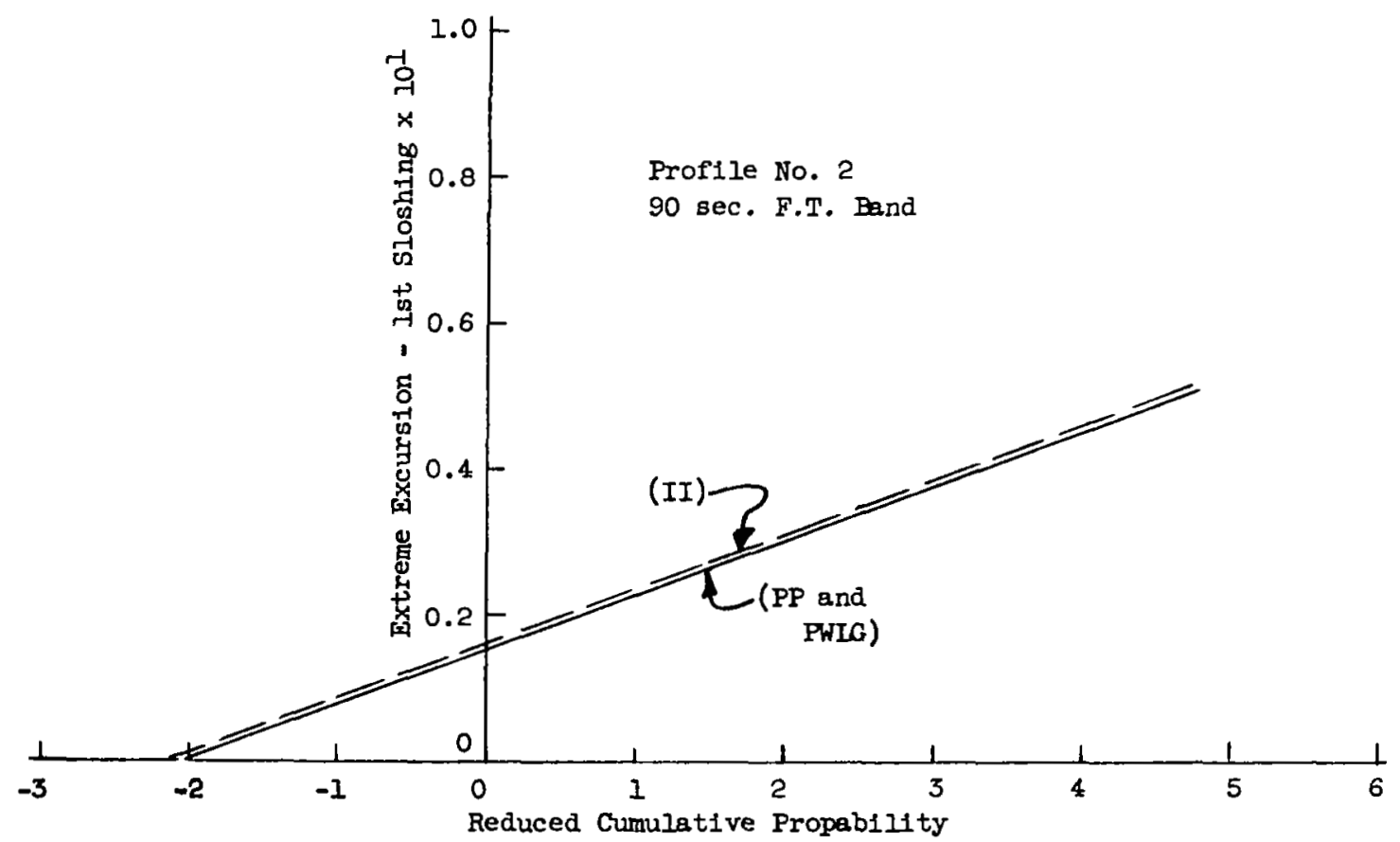

Fig. 80 - Extreme Excursion of 1st Sloshing vs. Reduced Cumulative Probability - $90 \mathrm{sec}$. F.T. 


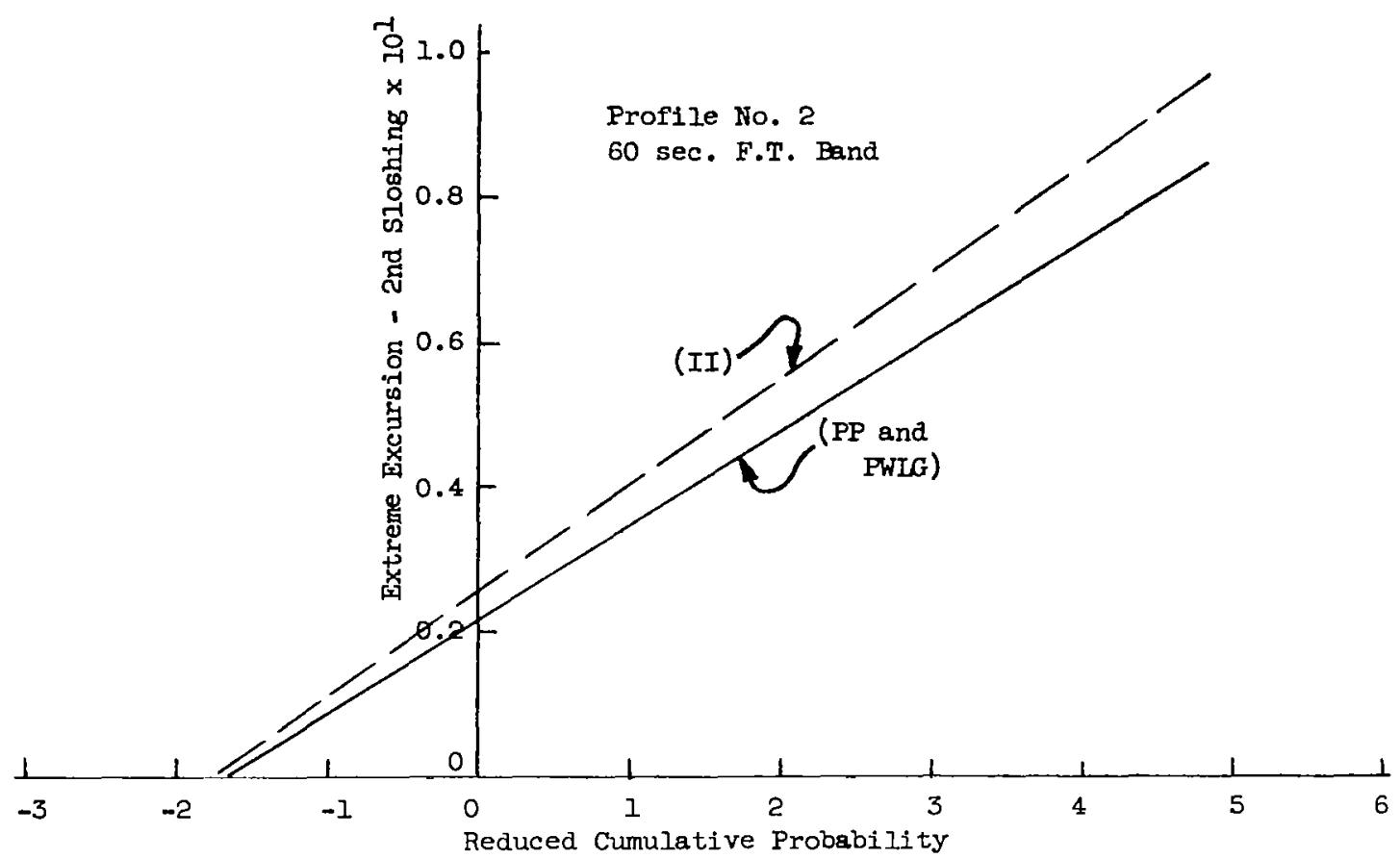

Fig. 81 - Extreme Excursion of 2nd Sloshing vs. Reduced Cumulative Probability - $60 \mathrm{sec}$. F.T.

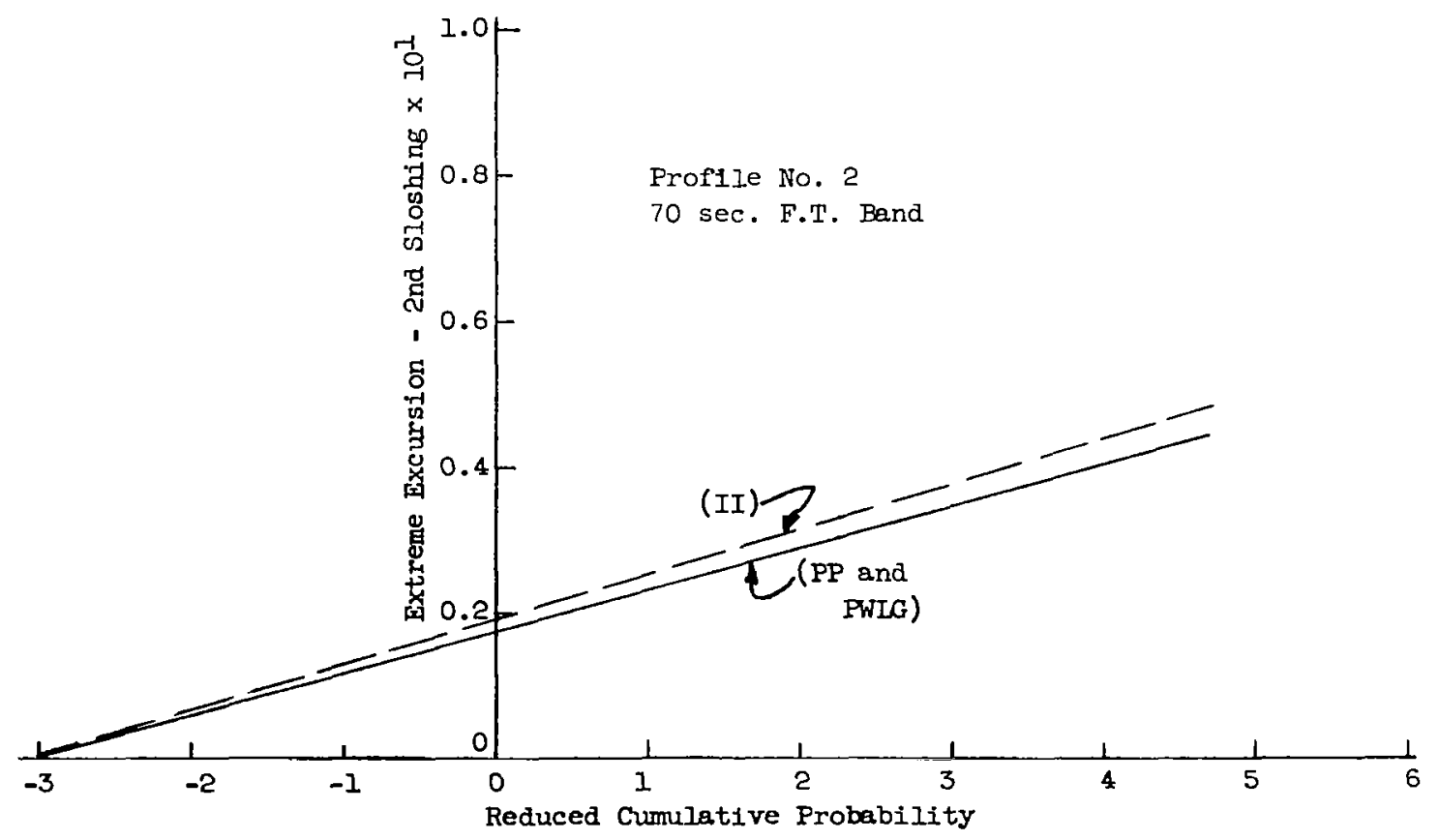

Fig. 82 - Extreme Excursion of 2nd Sloshing vs. Reduced Cumulative Probability - $70 \mathrm{sec}$. F.T. 


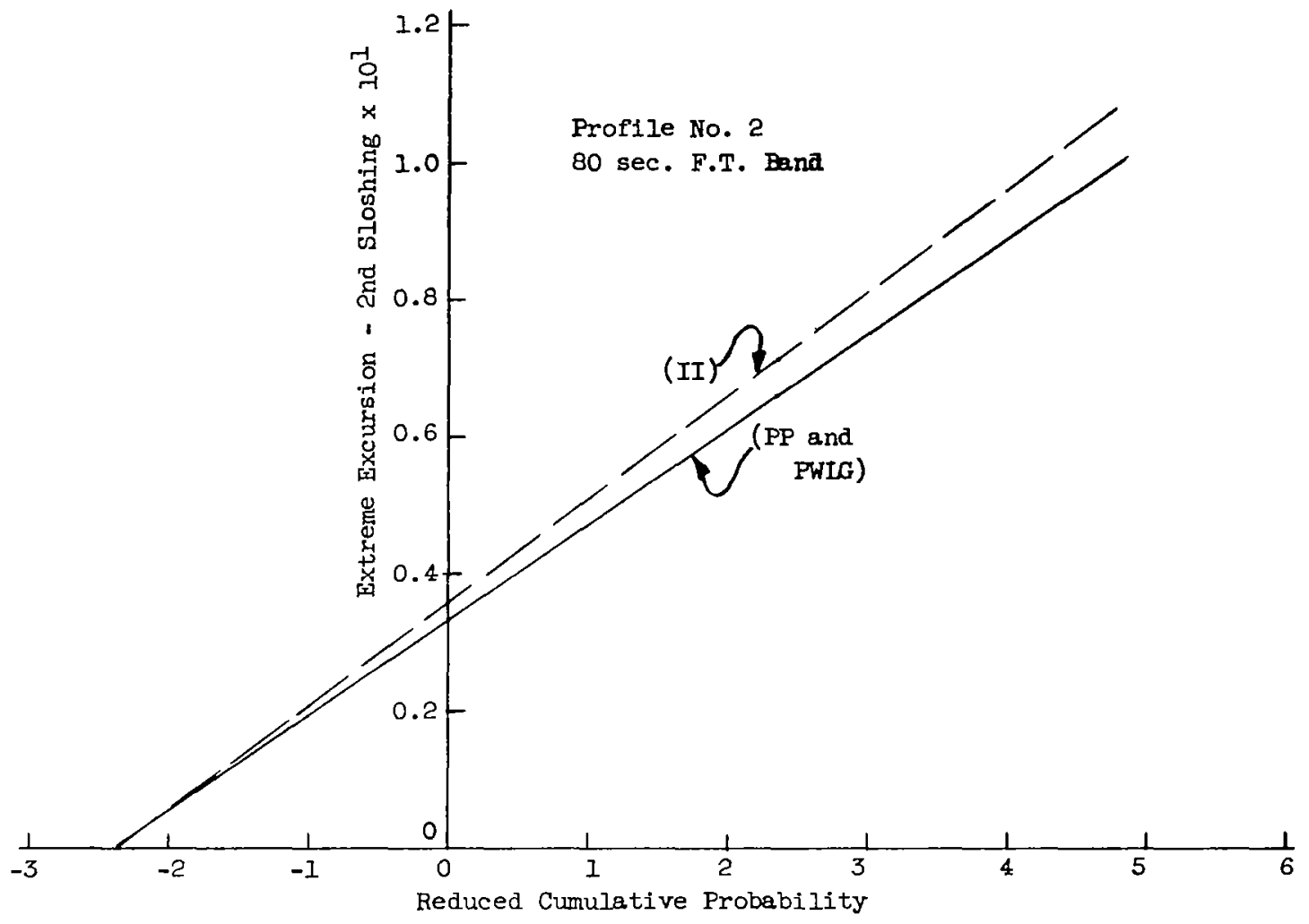

Fig. 83 - Extreme Excursion of 2nd Sloshing vs. Reduced Cumulative Probability - $80 \mathrm{sec}$. F.T.

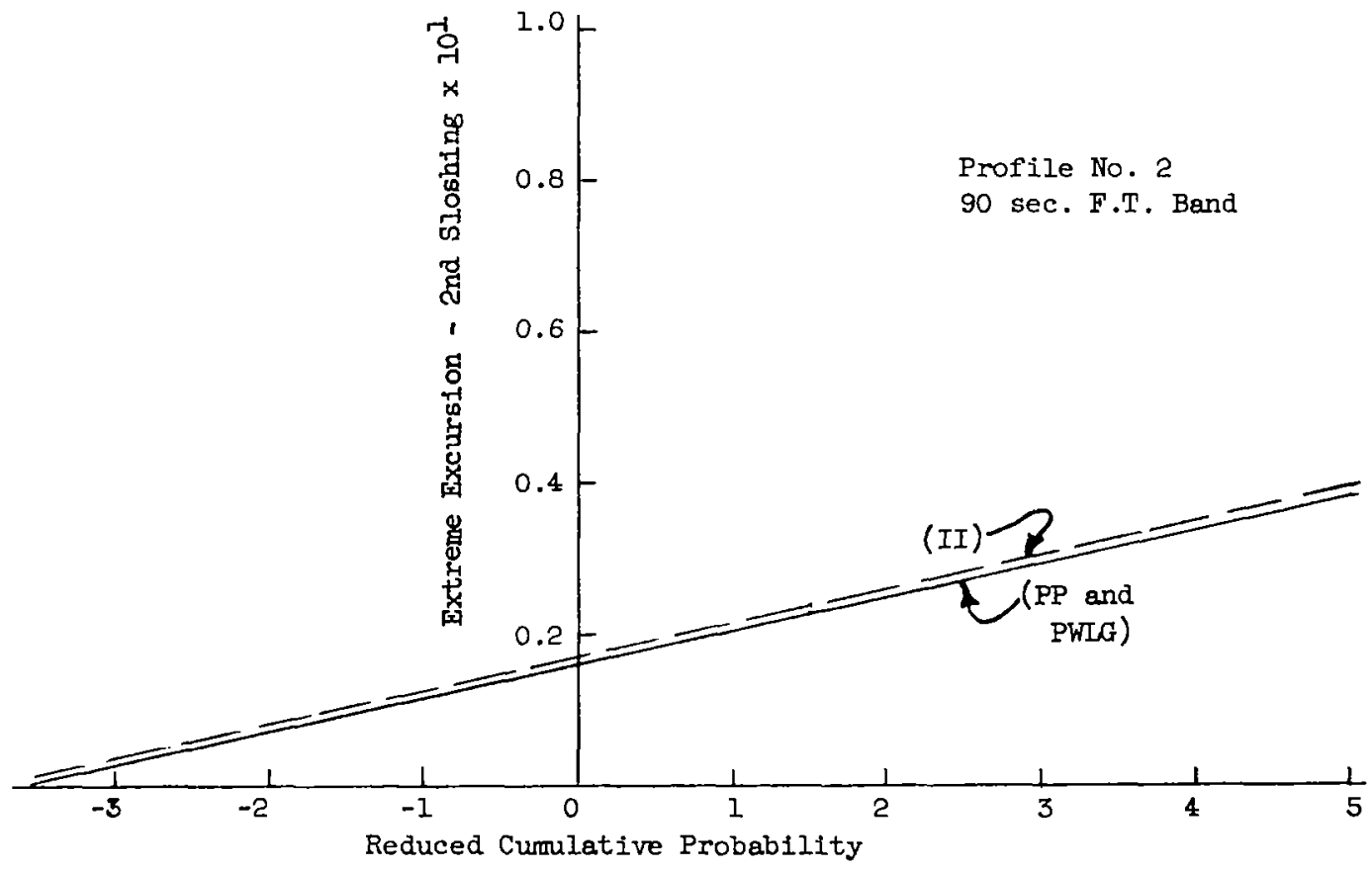

Fig. 84 - Extreme Excursion of 2nd Sloshing vs. Reduced Cumulative Probability - $90 \mathrm{sec}$. F.T. 


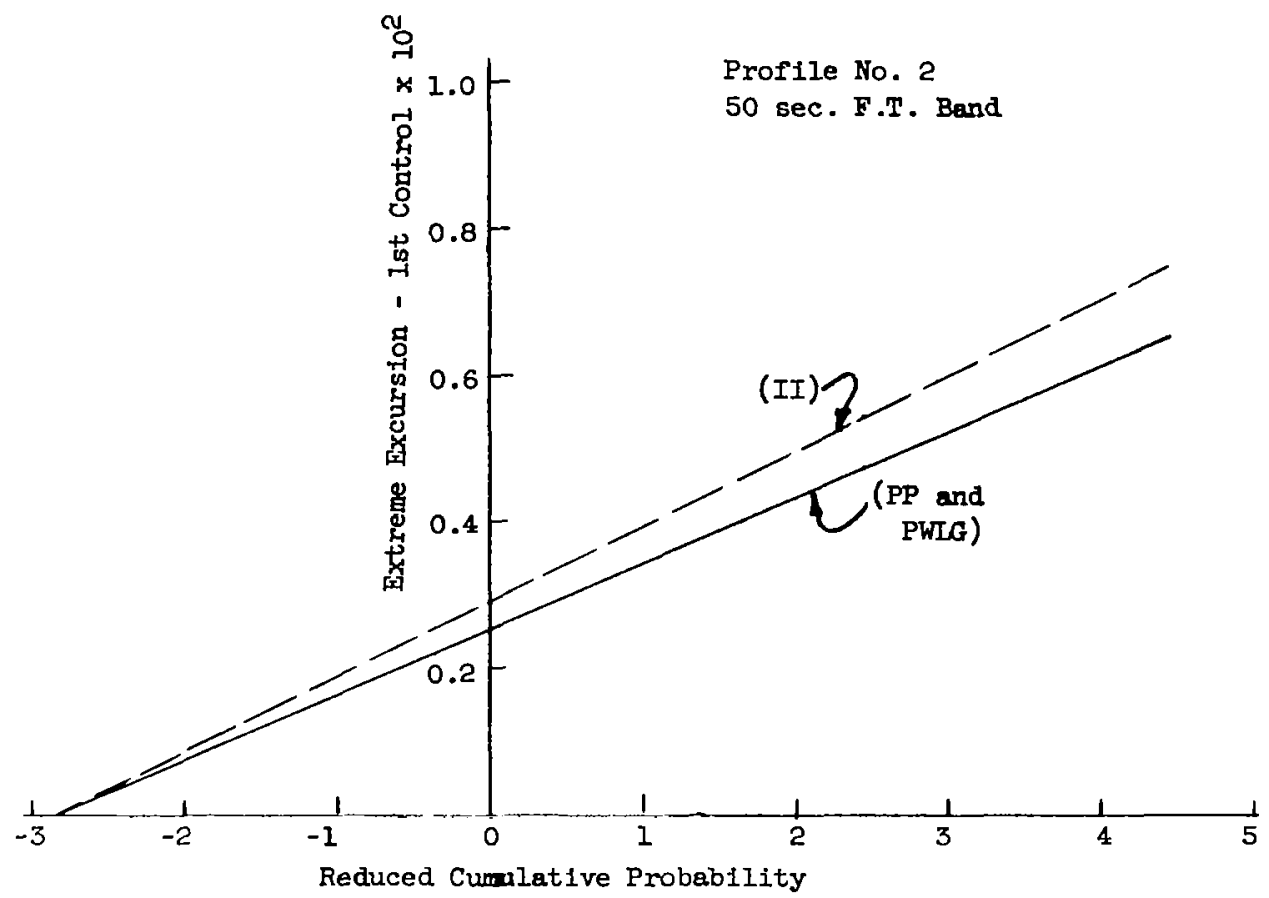

Fig. 85 - Extreme Excursion of lst Control vs. Reduced Cumulative Probability - $50 \mathrm{sec}$. F.T.

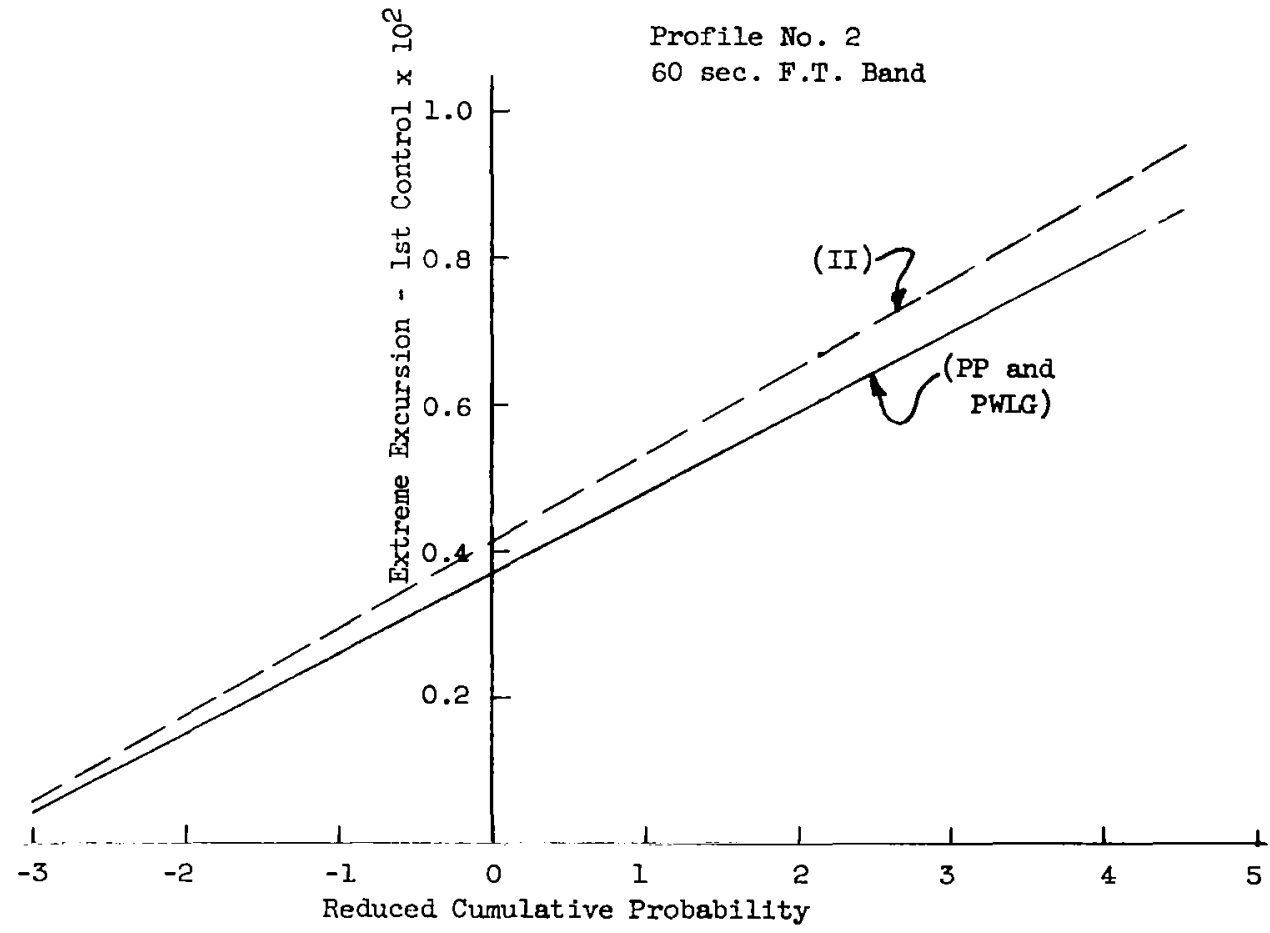

Fig. 86 - Extreme Excursion of lst Control vs. Reduced Cumulative Probability - $60 \mathrm{sec}$. F.T. 


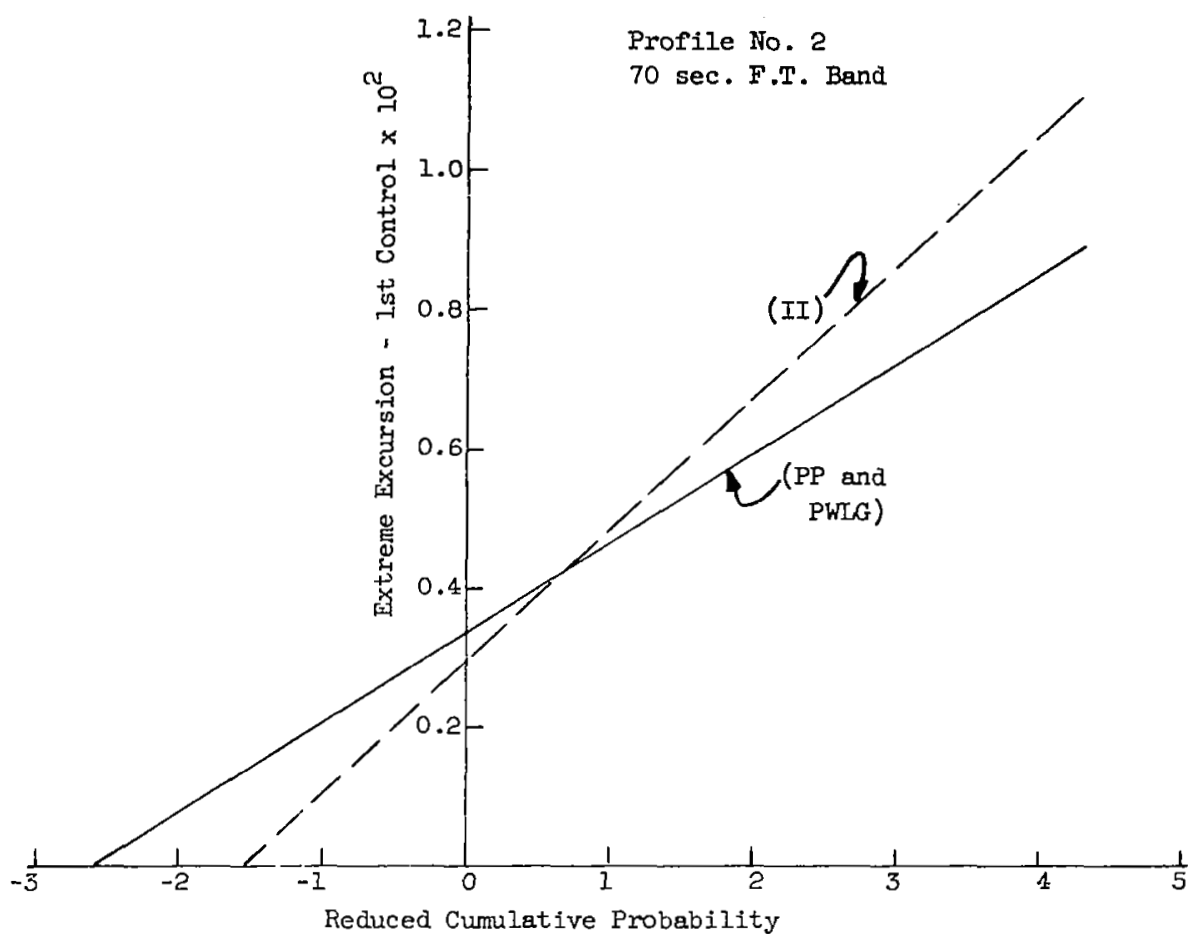

Fig. 87 - Extreme Excursion of 1st Control vs. Reduced Cumulative Probability - $70 \mathrm{sec}$. F.T.

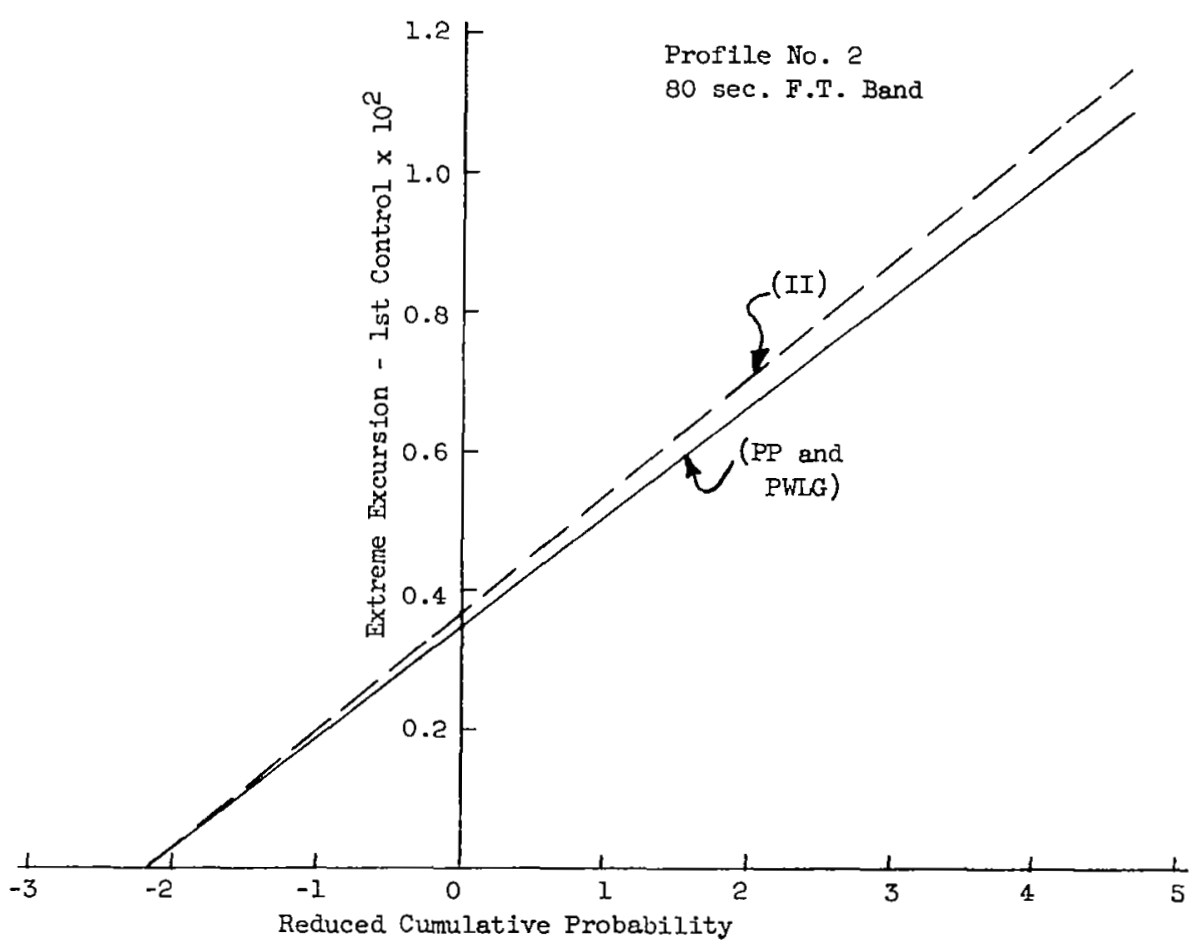

Fig. 88 - Bxtreme Excursion of lst Control vs. Reduced Cumulative Probability - $80 \mathrm{sec}$. F.T. 


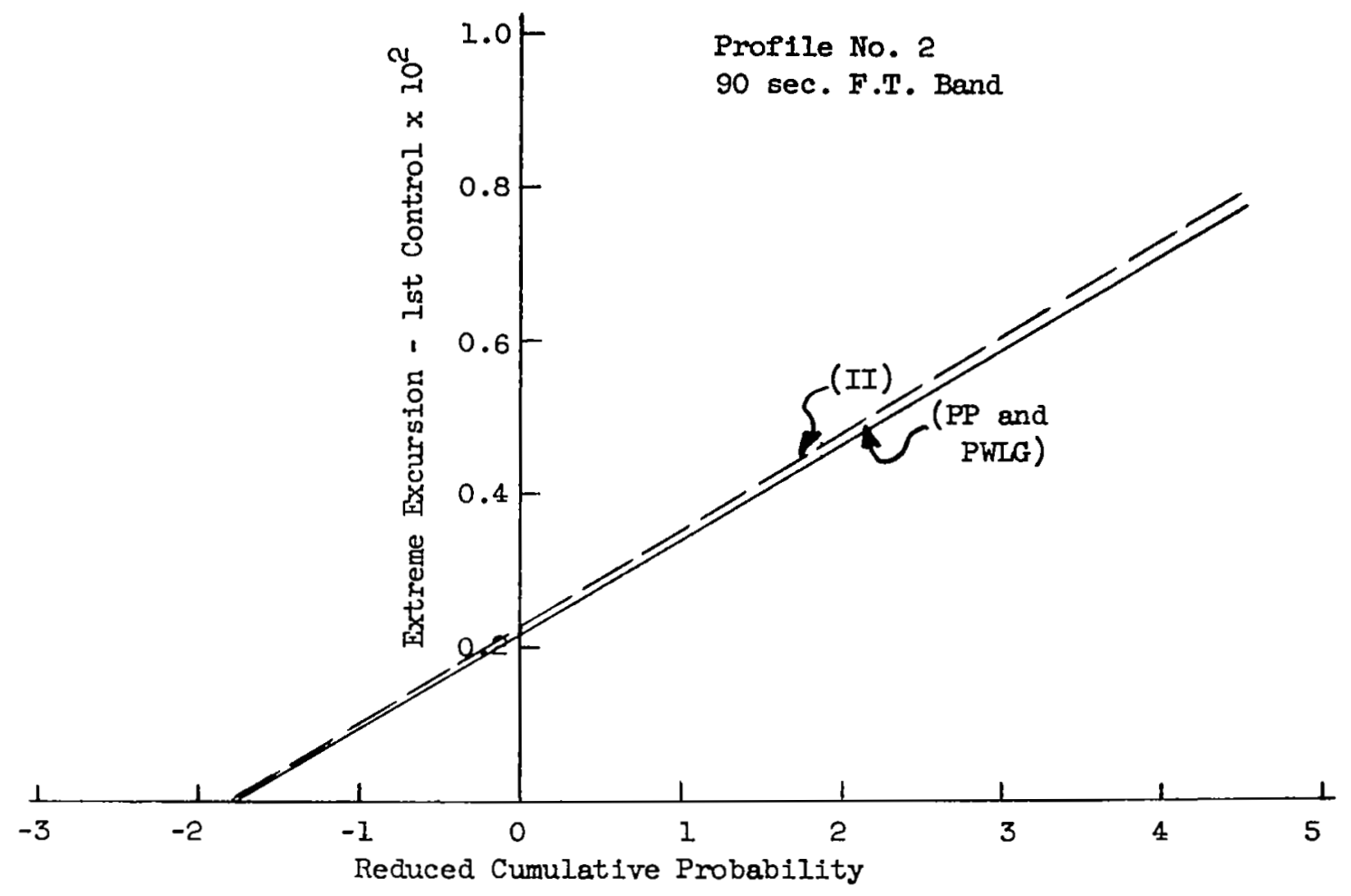

Fig. E9 - Extreme Excursion of Ist Control vs. Reduced Cumulative Probability - $90 \mathrm{sec}$. F.T. 


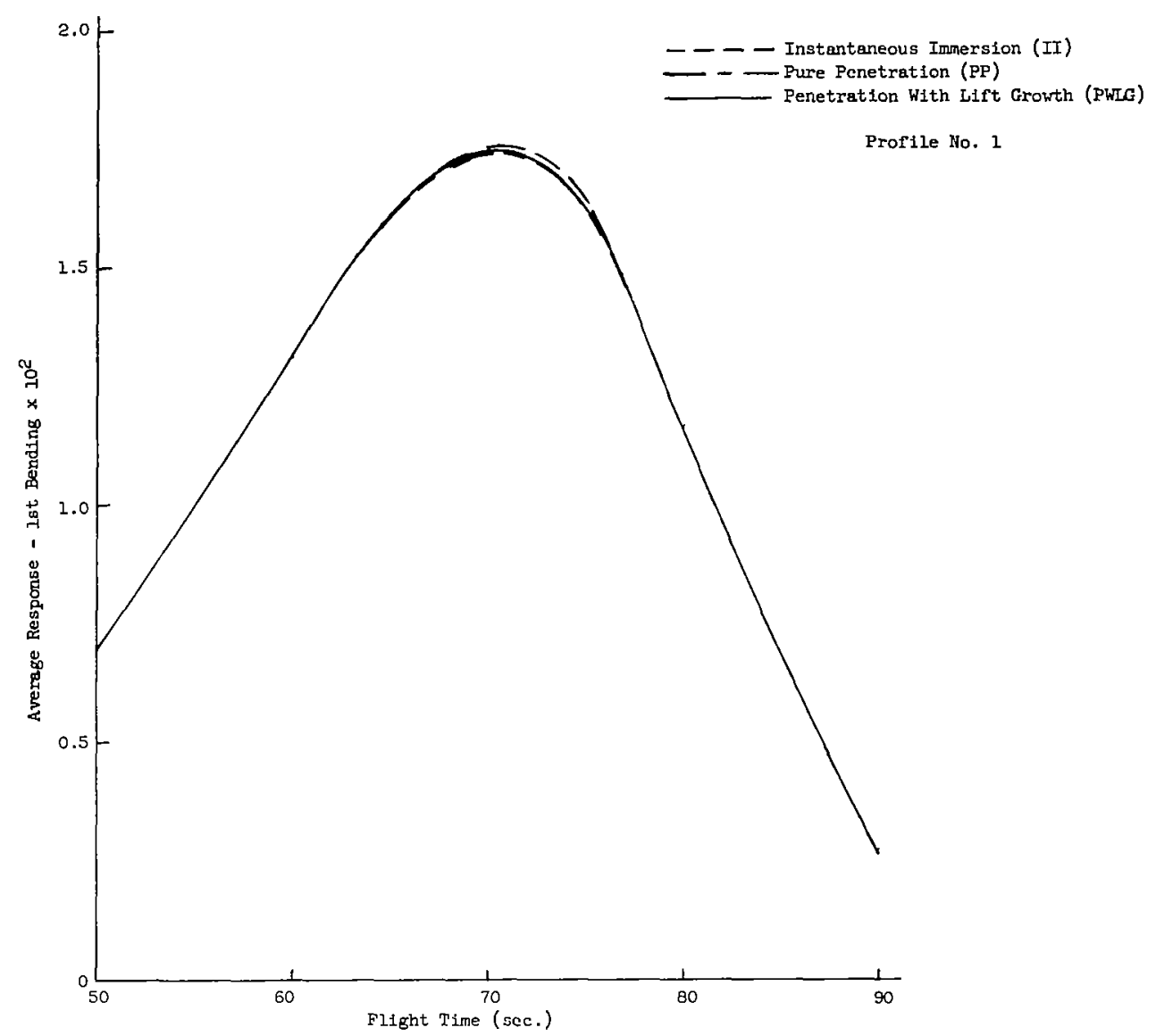

Fig. 90 - Average Response of Ist Bending vs. Flight Time

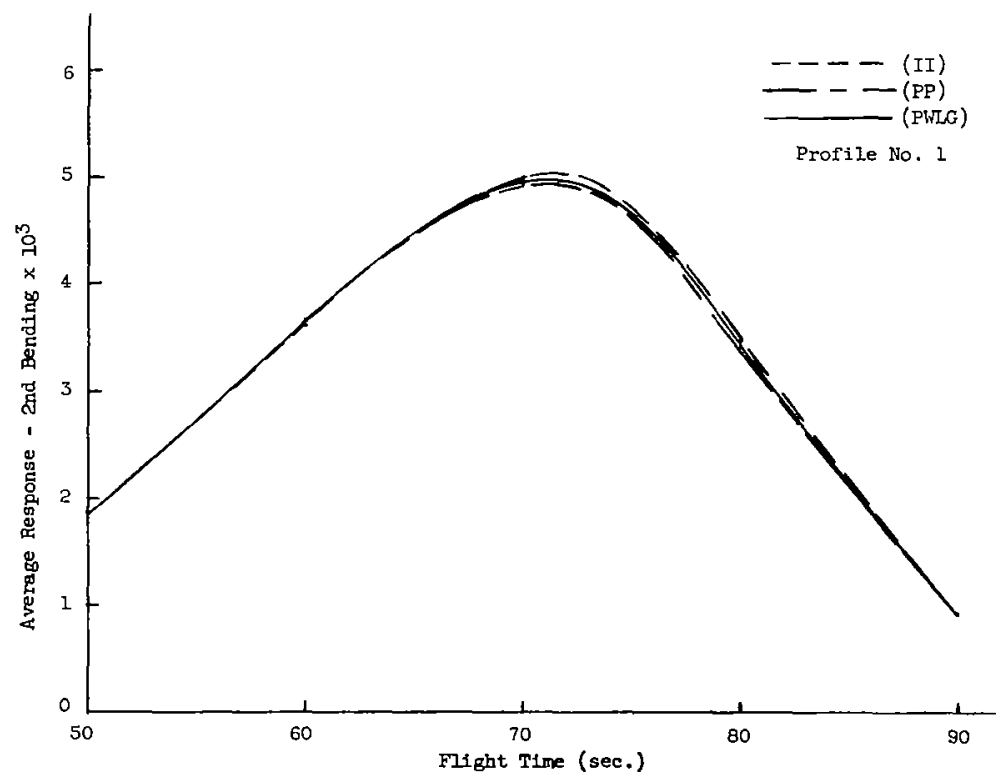

Fig. 91 - Average Response of 2nd Bending vs. Flight Time 


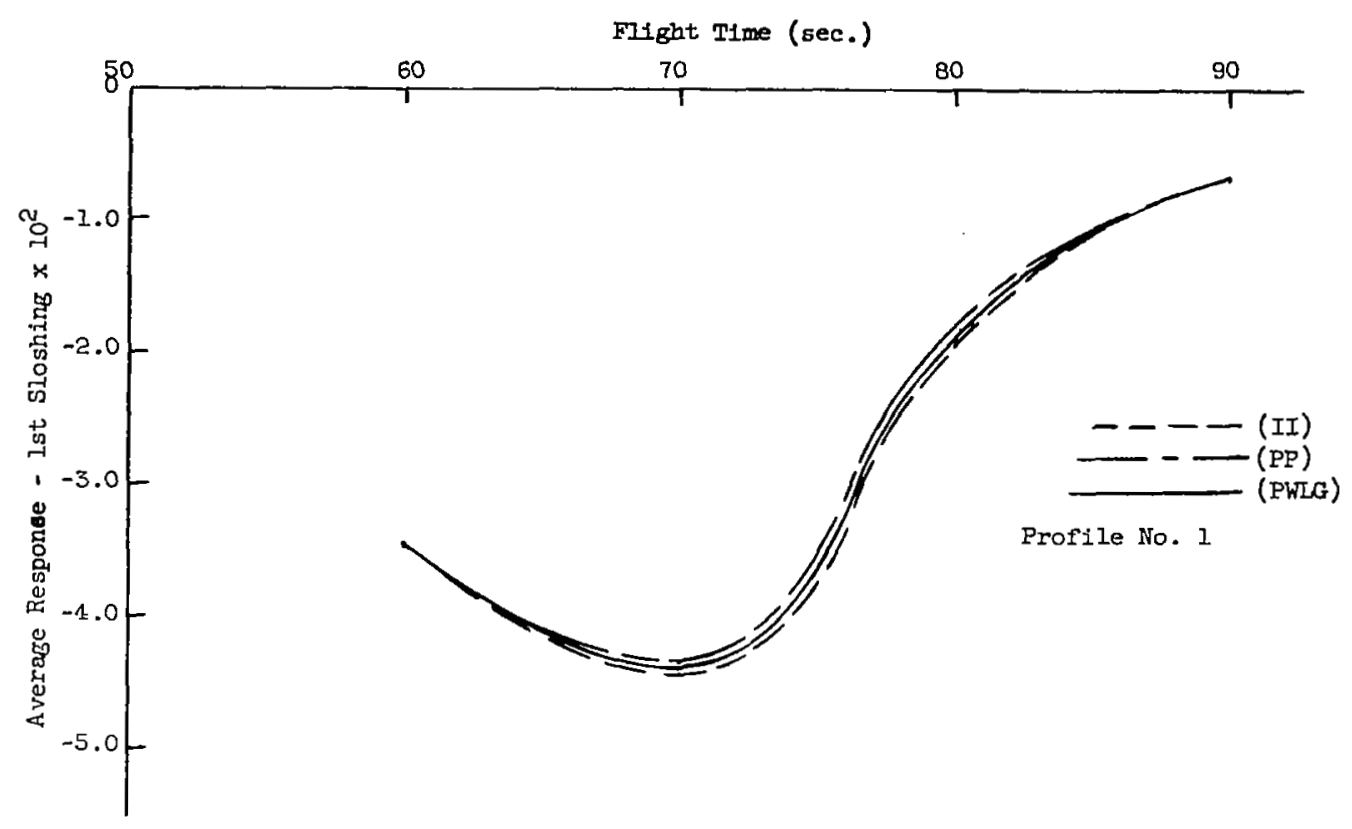

Fig. 92 - Average Response of lst Sloshing vs. FIight Time

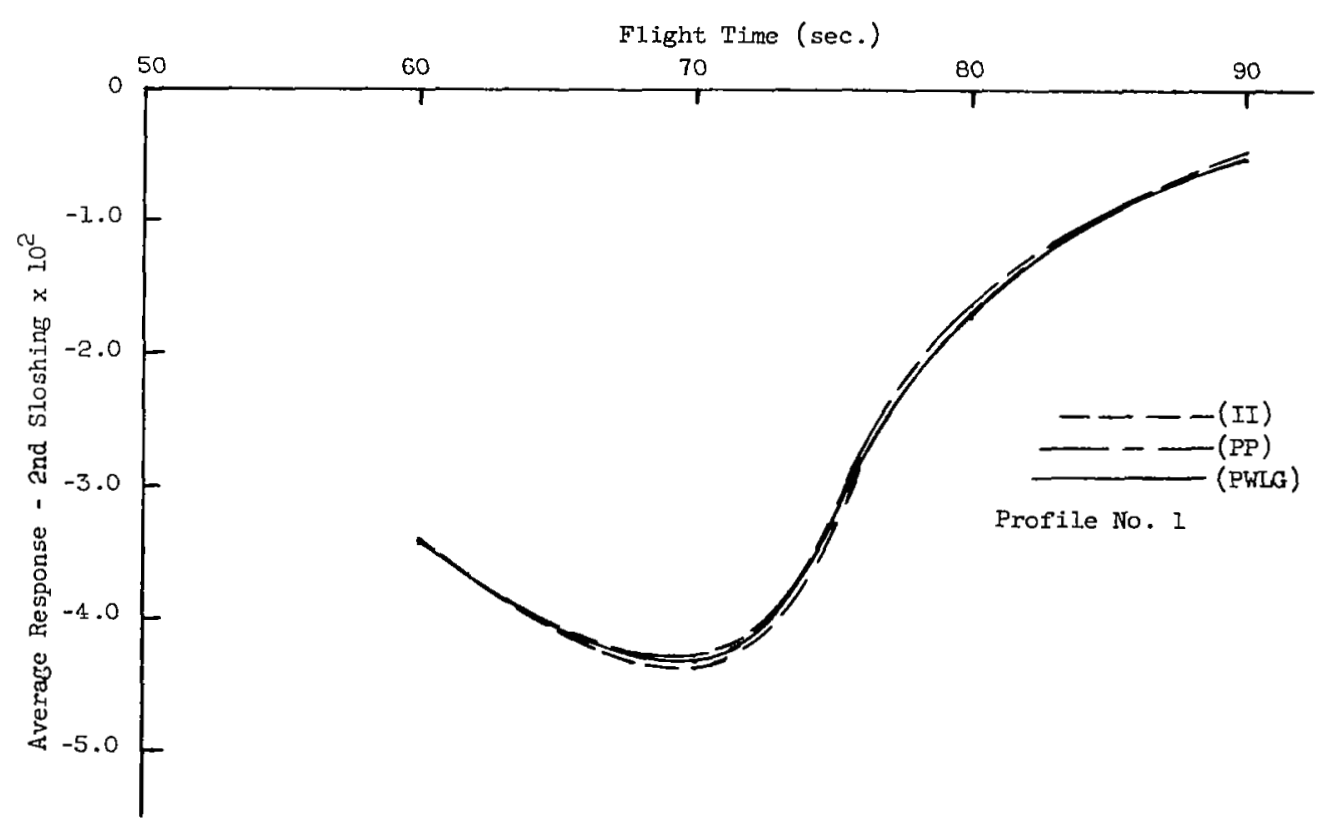

Fig. 93 - Average Response of 2nd Sloshing vs. Flight Time 
1

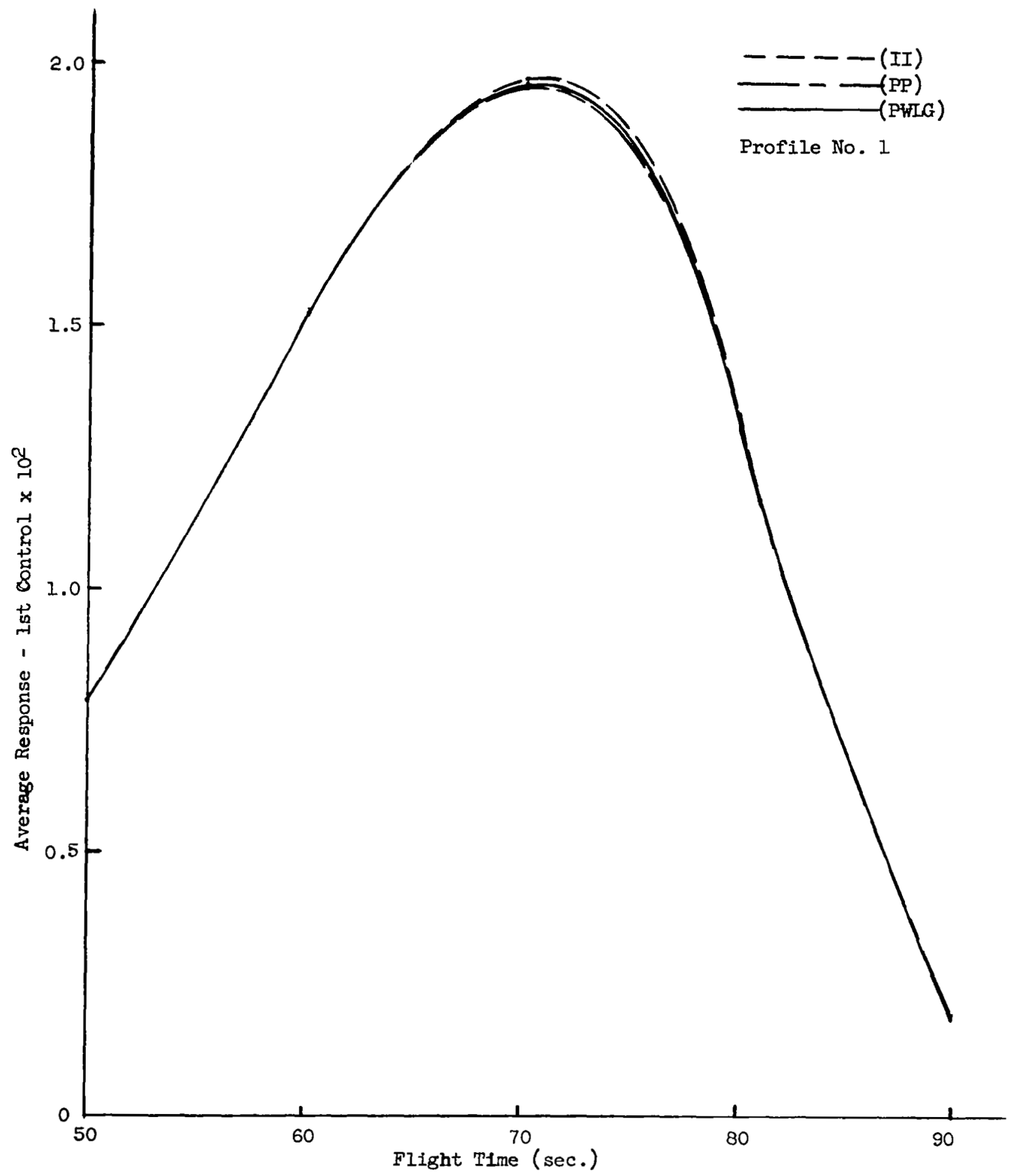

Fig. 94 - Average Response of lIst Control vs. Flight Time

$-138-$ 


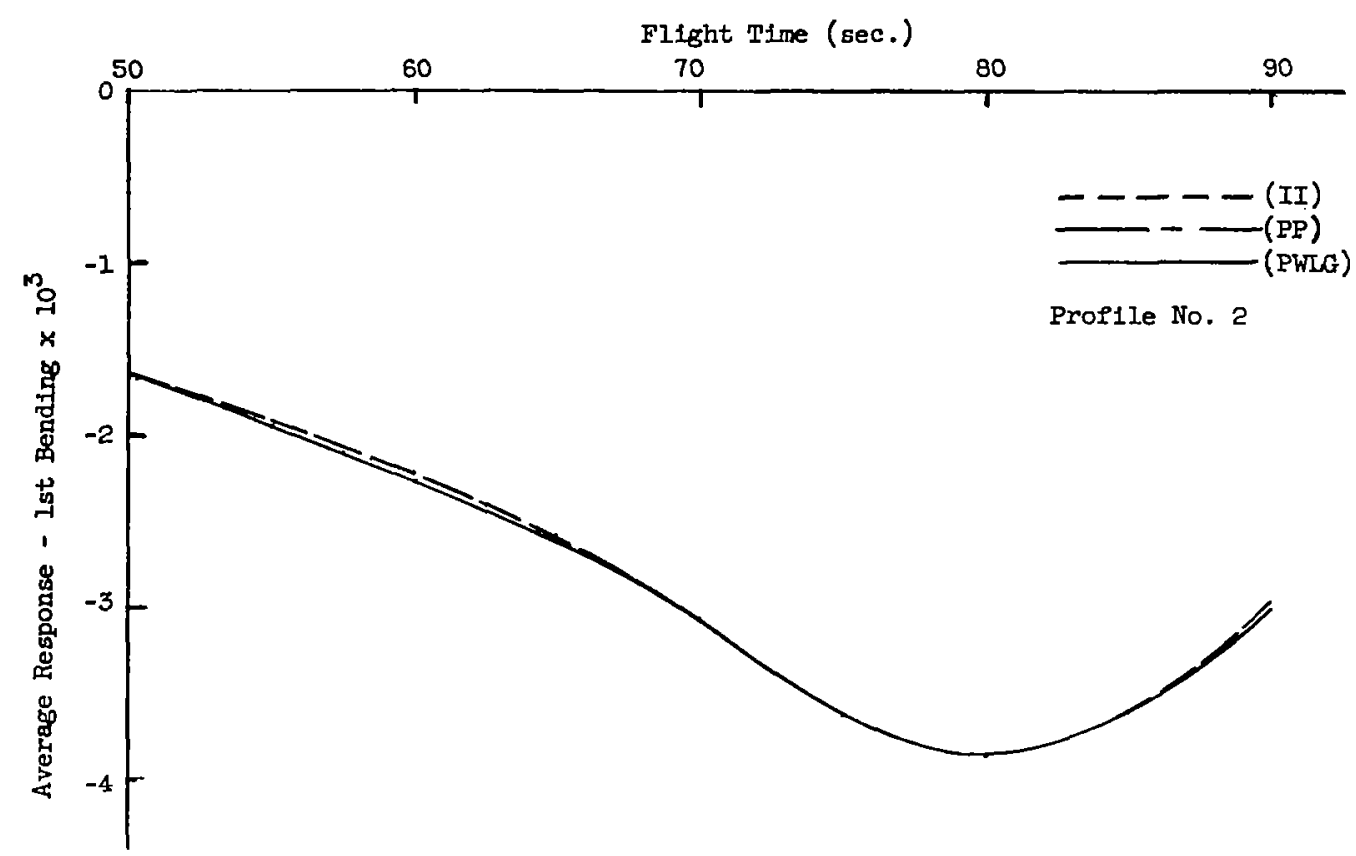

Fig. 95 - Average Response of lst Bending vs. Flight Time

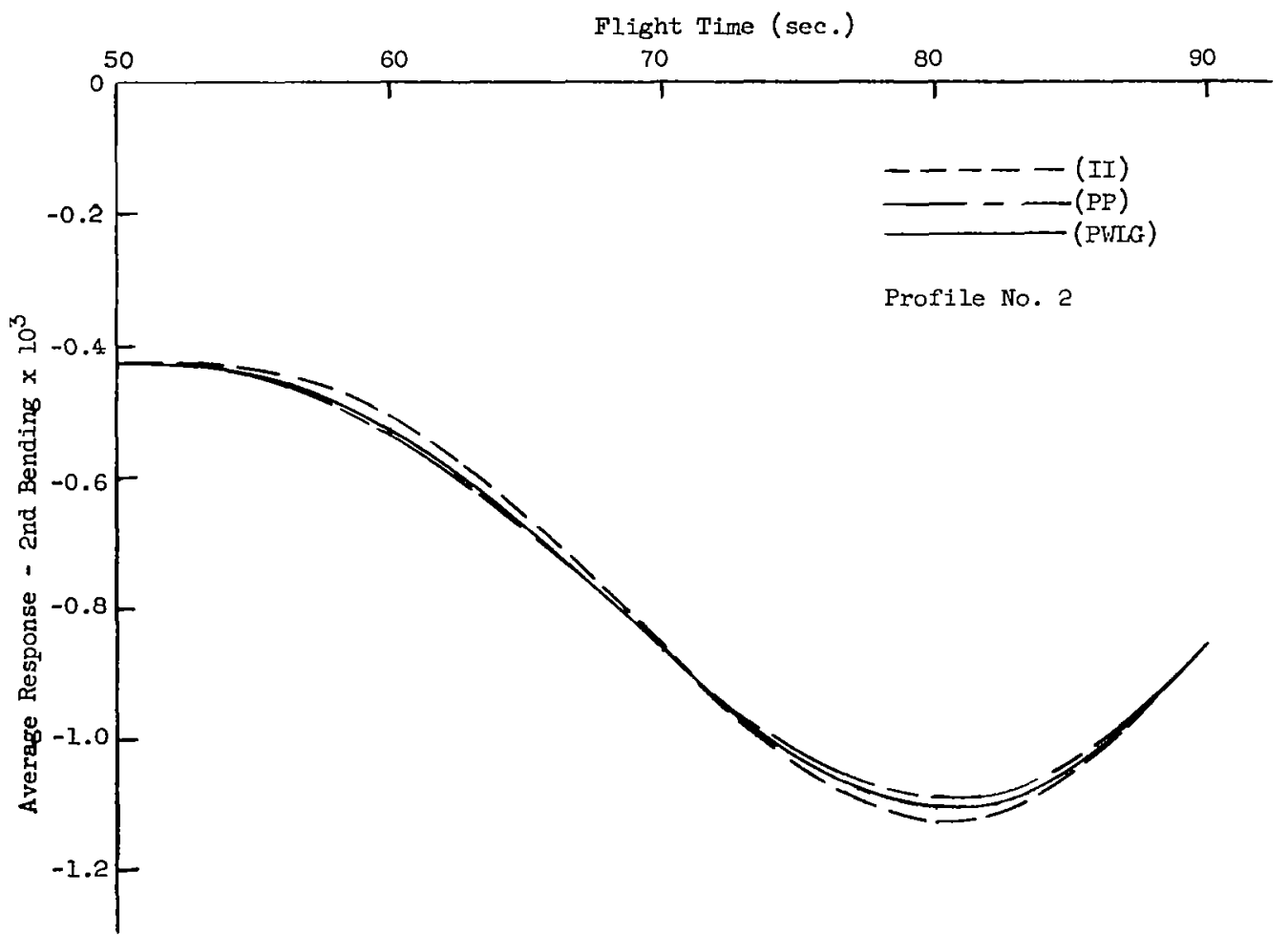

Fig. 96 - Average Response of 2nd Bending vs. Flight Time 


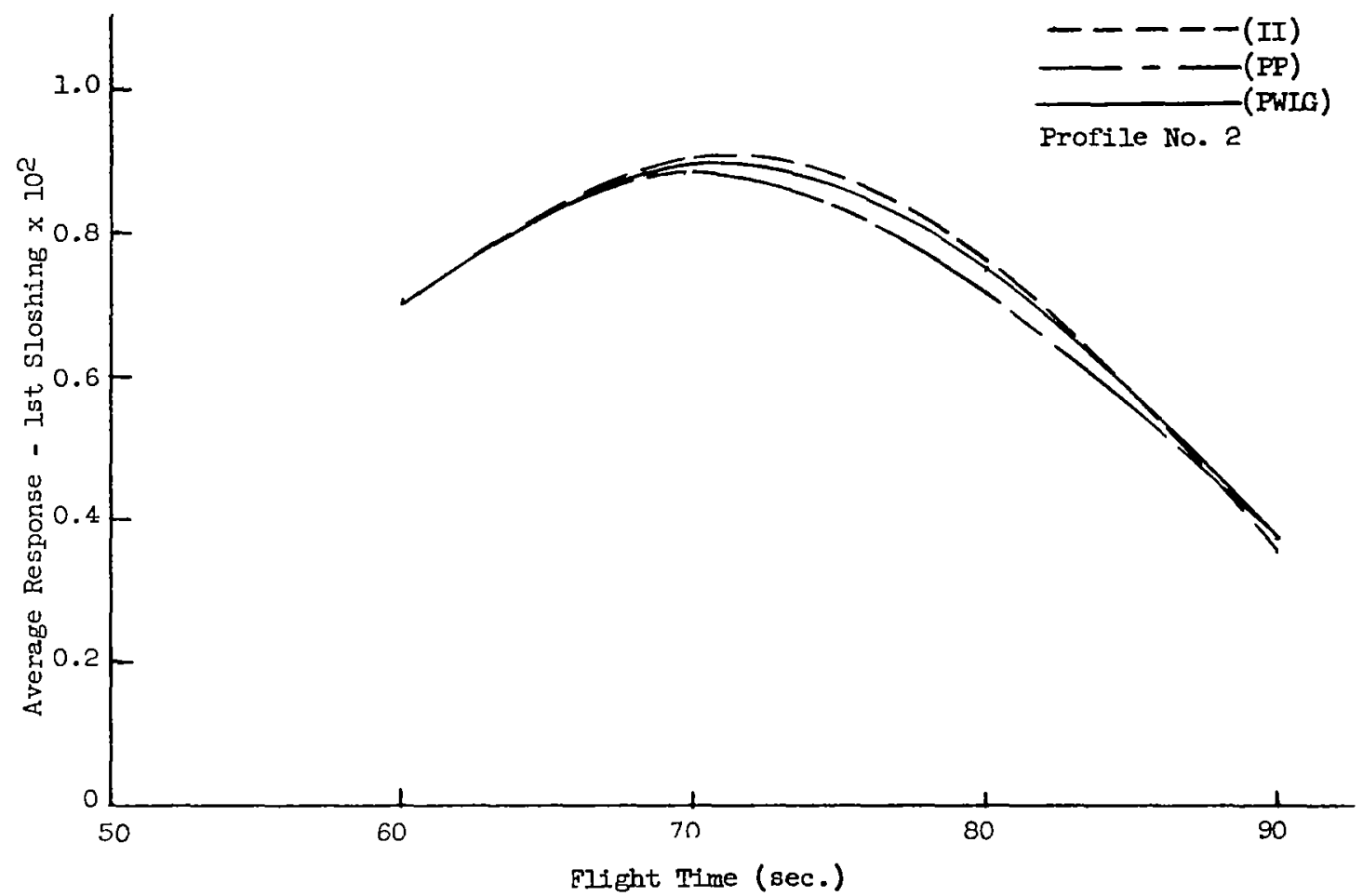

Fig. 97 - Average Response of lst Sloshing vs. Flight Time

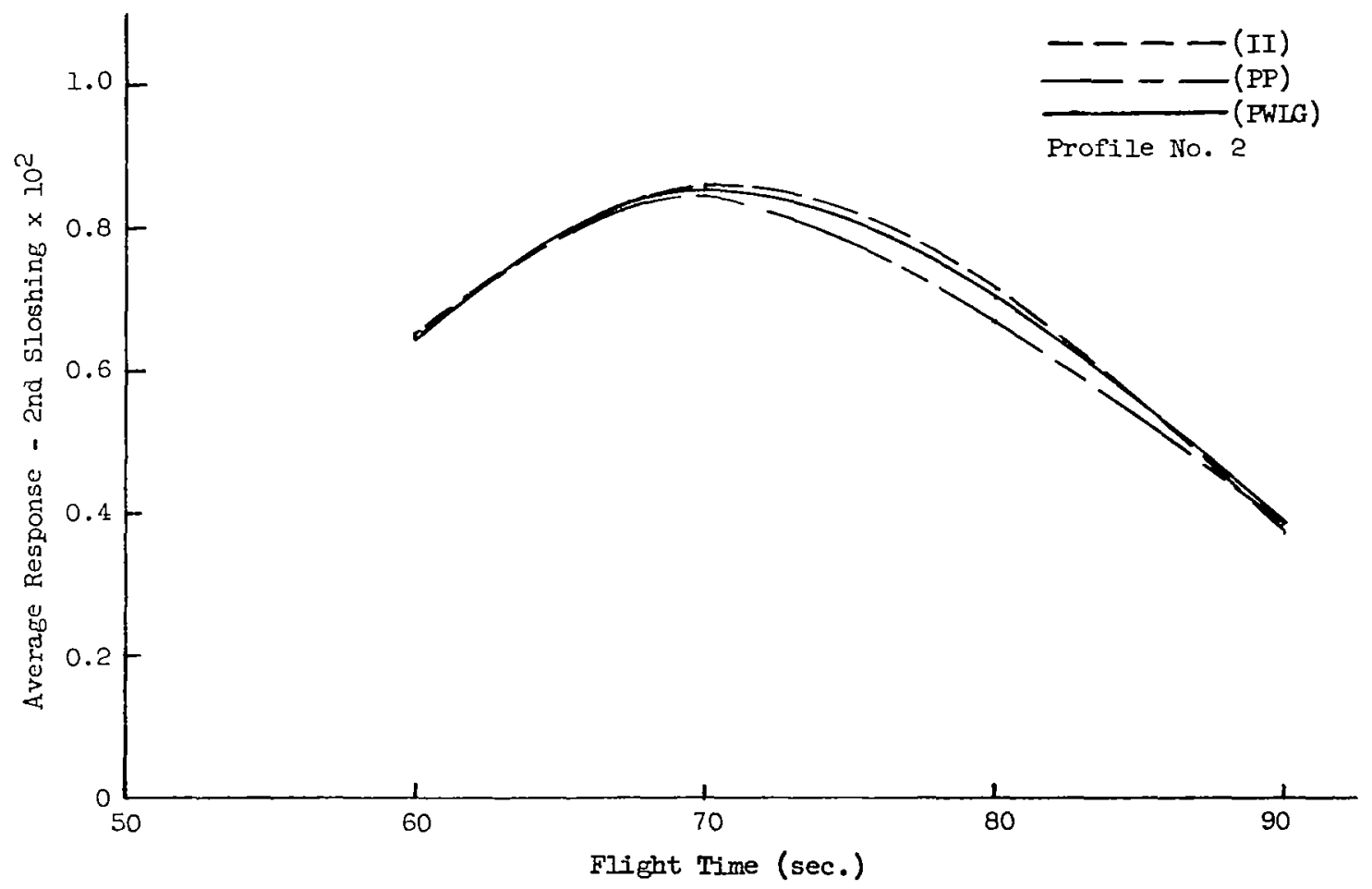

Fig. 98 - Average Response of 2nd Sloshing vs. Flight Time 


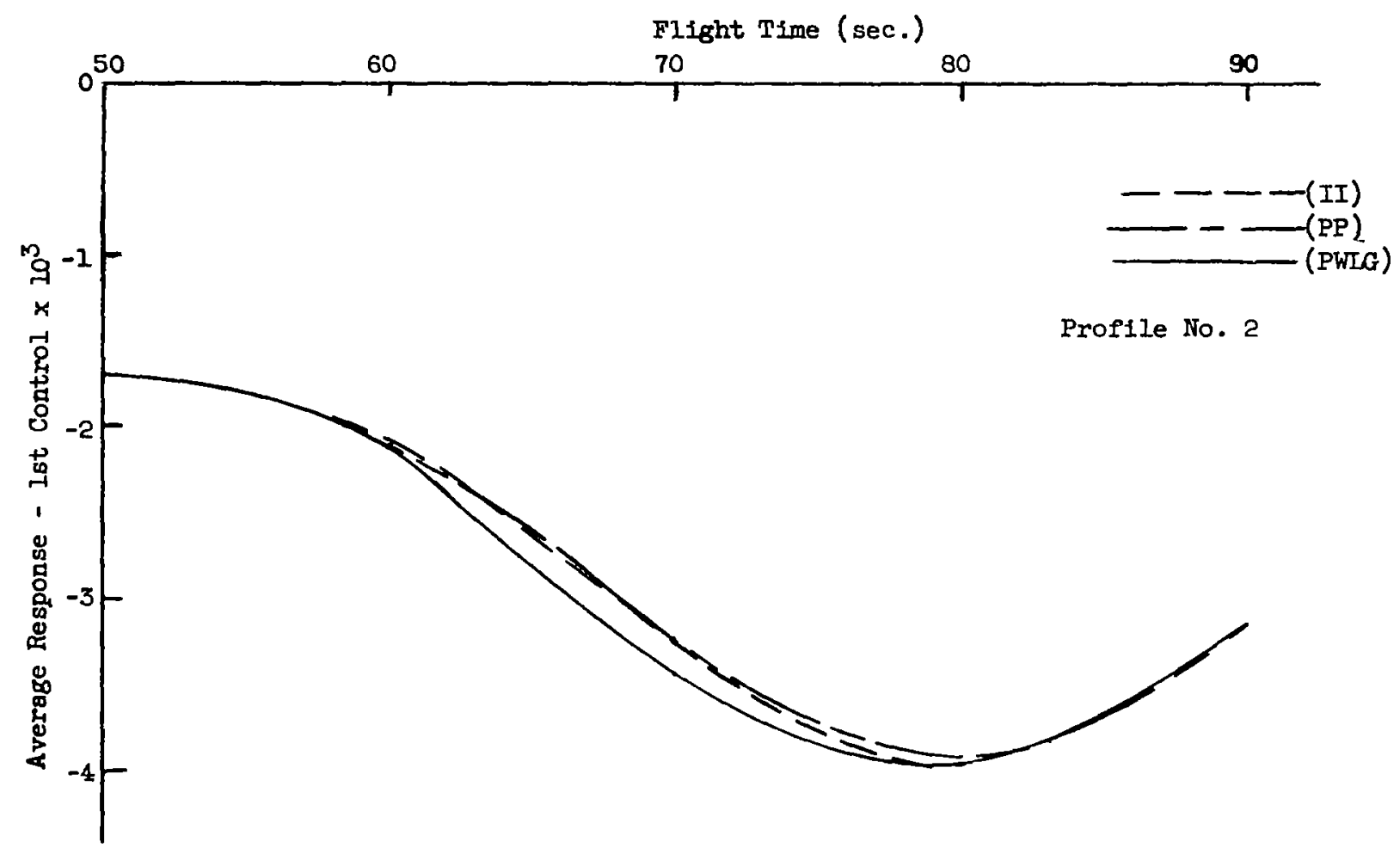

Fig. 99 - Average Response of Ist Control vs. Flight Time 